\title{
MONOLITHIC CIRCUIT TEST STANDARDS
}

By George O. Compton and Joseph R. Adame

October 1967

Prepared under Contract No. NAS 8-21199 by FAIRCHILD SEMICONDUCTOR, DIVISION OF FAIR CHILD CAMERA AND INSTRUMENT CORPORATION Mountain View, California

George C. Marshall Space Flight Center

NATIONAL AERONAUTICS AND SPACE ADMINISTRATION 


\title{
PRECEDING PAGE BLANK NOT FILMED.
}

\author{
SUMMARY
}

This final report covers the work performed under Contract NAS 8-21199. This contract with Fairchild Semiconductor, a Division of Fairchild Camera and Instrument Corporation, was for the preparation of "Monolithic Circuit Test Standards" for use in Line Certification of monolithic circuits. Dr. A. M. Holladay, of the Astrionics Laboratory, George C. Marshall Space Flight Center, National Aeronautics and Space Administration, Huntsville, Alabama, was the Technical Contract Monitor.

Mr. George O. Compton was the principal investigator during the period of this contract. Mr. Joseph R. Adame also worked on the contract.

No New Technology was developed during the work on this contract.

\section{INTRODUCTION}

The following specifications have been prepared after a survey of Resea rch and Development and Manufacturing Facilities. Methods, except for certain proposals, are as used in day-to-day operations. Proposed tests have been presented after consultation with scientists and engineers most knowledgeable in the areas covered.

Items included in the Contract scope of work, which are not covered in this series of standards, include. 


\section{Water $-\mathrm{CO}_{2}$ and Organic content. \\ 2. Substrate - Inclusions. \\ 3. Mounting - Centrifuge and Radiographic Evaluation. \\ 4. Electrical Tests - Bipolar Transistor parameters, except storage time and alpha cut-off frequency.}

Present technology of hyper-pure water evaluation is not sophisticated enough to have evolved meaningful tests for either $\mathrm{CO}_{2}$ or Organic content.

Inclusions in substrate material will be most readily apparent after epitaxy.

MIL-STD-202, Method 212 and MIL-STD-750, Method 2006 are deemed adequate for evaluation of mounting and packaging by centrifuge.

No improvement upon MIL-STD-202, Method 209, is presently available for radiographic examination.

MIL-STD-750, Methods 3001-4151 are adequate for process control, except that the storage time and alpha cut-off frequency are difficult to measure prior to dicing. Measurement of stored charge, $\mathrm{Q}_{\mathrm{bx}}$, is suggested as a substitute for these A-C tests.

During the course of the Contract, it has become apparent that additional research is required in several areas. Evaluation of water for use in semiconductor manufacture would be a fertile area of effort.

Present radiographic techniques leave much to be desired when examining devices manufactured with low mass materials. Aluminum wire, for example, is impossible to detect in X-Ray photographs of integrated circuits.

Current methods for detecting gross leaks utilize the observation of bubbles from defective devices. This is both a slow and subjective technique. An improved procedure should be developed which is more definitive and quantitative. One approach would be the introduction of liquid into the package which could later be detected in radiflow-type equipment. 
TABLE OF CONTENTS

TEST

Substrate Bulk Resistivity

Substrate Orientation

Substrate Thickness

Substrate Parallelism

Substrate Flatness

Substrate Crystal Perfection

Substrate Conductivity Type

Substrate Surface Finish

Substrate Width or Diameter

Water Resistivity

Water Solids

Water Bacteria Count

Photoresist Viscosity (ASTM)

Photoresist Viscosity (Torque)

Photoresist Solids Residue

Photoresist Surface Tension
METHOD NO.

101

102

103

104

105

106

107

108

109

110

111

112

113

114

115

116 


\section{TABLE OF CONTENTS \\ (Continued)}

TEST

METHOD NO.

Thickness of Epitaxial Layer (stacking fault)

Thickness of Epitaxial Layer (IR) EIR

118

Thickness of Epitaxial Layer (Nomarsky)

Resistivity of Epitaxial Layer (Four-point Probe)

Epitaxial Layer Surface Defects

Epitaxial Process (Substrate Temperature)

Thickness of Passivating Layer (Tolansky)

Thickness of Passivating Layer (Polychromatic)

Thickness of Passivating Layer (Monochromatic)

Stability of Passivating Layer ( $\mathrm{C}-\mathrm{V}$ Curve)

Pinholes in Passivating Layer

Photoresist Process (Minimum Line Width)

Photoresist Process (Thickness of Film)

Photoresist Process (Pinholes in Film)

Photoresist Process (Light Intensity)

Photoresist Process (Spinner RRM)

132

Junction Formation (Depth)

Junction Formation (Base Width)

Junction Formation (Sheet Resistance) 
Junction Formation (Temperature Profile) 136

$\begin{array}{ll}\text { Junction Formation (Gas Profile) } & 137\end{array}$

$\begin{array}{ll}\text { Diode Isolation (Breakdown Voltage) } & 138\end{array}$

Dielectric Isolation (Breakdown Voltage) 139

$\begin{array}{lr}\text { Isolation Leakage } & 140\end{array}$

Metallization Thickness (Tolansky) $\quad 141$

Metallization Thickness (Sheet Resistance) 142

$\begin{array}{ll}\text { Metallization Adhesion } & 143\end{array}$

Stability of Metallization $\quad 144$

$\begin{array}{ll}\text { Specific Resistivity of Metallization } & 145\end{array}$

$\begin{array}{ll}\text { Quality of Ohmic Contact } & 146\end{array}$

Cross-Sectional Uniformity of Metallization $\quad 147$

Pull Strength of Interconnection Bonds $\quad 148$

Moisture Content (Dry Box Sealing) $\quad 149$

$\begin{array}{ll}\text { Moisture Content (Belt Furnace Sealing) } & 150\end{array}$

Hermaticity, Fine and Medium Leak $\quad 151$

Hermaticity, Gross Leak 152

Sealing, Defects in Glass (Can Package) 153

Sealing, Defects in Glass (Flat Package) 154 
TABLE OF CONTENTS

(Continued)

TEST

Stored Charge, Bi polar Transistor $\left(Q_{b x}\right)$

Breakdown Voltage ( $\mathrm{BV}_{\text {DSS }}$ ) Drain to Source

Breakdown Voltage, Source to Gate ( $\mathrm{BV}_{\mathrm{GSS}}$ )

Drain to Source Cutoff Current ( DSS )

Source to Gate Cutoff Current (I ${ }_{G S S}$ )

Gate to Source Threshold Voltage ( $\mathrm{V}_{\mathrm{GST}}$ )

Gate Leakage Resistance $\left(\mathrm{R}_{\mathrm{GS}}\right)$

Dynamic Drain-Source Resistance (Rd(on))

Static Transconductance $\left(\mathrm{g}_{\mathrm{m}}\right)$

Direct Inter-terminal Capacitance $\left(\mathrm{C}_{\mathrm{GS}}, \mathrm{C}_{\mathrm{GD}}, \mathrm{C}_{\mathrm{DS}}\right)$
METHOD NO.

155

156

157

158

159

160

161

162

163

164 
Method 101

Substrate Bulk Resistivity

(Four Point Probe)

1. Purpose

This method may be used to determine the resistivity of the substrate. A four-point probe is used to obtain sheet resistance for a material of known thickness, which yields resistivity:

$$
\begin{aligned}
& \rho=A \frac{V}{I} \\
& A=F(W, S) \\
& \text { if } \mathrm{W}<\mathrm{S} \\
& \rho=4.53 \mathrm{~W} \frac{\mathrm{V}}{\mathrm{I}} \\
& \text { Where: } \quad W=\text { Substrate Thickness } \\
& S=\text { Probe Spacing } \\
& \frac{V}{I}=\text { Sheet Resistance of the Layer }
\end{aligned}
$$

2. Apparatus

2. 1 Four-Point Probe
A. M. Fell Co.

(or equivalent)

2. 2 Power Supply, Constant Current

0.1 , 1. 0, 10mA Ranges

(any source)

\section{3 Voltmeter, Digital \\ Fairchild 7100 \\ (or equivalent)}


3. Materials

.3. 1 Glass Microscope Slides, 1" $\times 3 "$

4. Procedure

4. 1 Place the wafer to be measured on the glass slide under the four point probe head. Center the wafer so that no probe point is closer than $1 / 8^{\prime \prime}$ from the edge of the wafer. See Figure 1.

4. 2 Set the constant current selector to $0.01 \mathrm{ma}$.

4. 3 Turn the constant current generator switch on.

4. 4 Screw the four point probe down on the wafer using the end ratchet until the proper contact causes the ratchet to slip.

4. 5 Check to see if the constant current meter is indicating full scale. If not, raise the probe and repeat step 4. 4 .

4. 6 Switch the polarity of the current generator from plus to minus and observe the current reading for any variation.

4. 7 If full scale current readings are obtained in both positions, (i. e., plus and minus), read the indicated $V / I$ on the voltmeter.

4. 8 The V/I readings should be within $10 \%$ of each other when the polarity of the current generator is reversed. If this is not so, release the pressure on the probe head $1 / 4$ turn of the ratchet at a time until the readings do agree within $10 \%$.

4. 9 Take three V/I readings on each check wafer, average and record. See Figure 2 for example.

\section{Calibration}

5. 1 Probe Spacing

5. 1. 1 Cover a $1^{\prime \prime} \times 3^{\prime \prime}$ glass microscope slide with aluminum foil. 
5. 1. 2 Obtain an impression of the four point probe points.

5. 1.3 Measure spacing with any optical comparator.

5. 1. 4 Adjust spacing per probe manufacturer's instructions.

\section{Accuracy}

Obtainable accuracy is $\pm 10 \%$.

\section{Test Frequency}

This test should be performed on every substrate. 


\section{FOUR POINT PROBE - SET-UP}

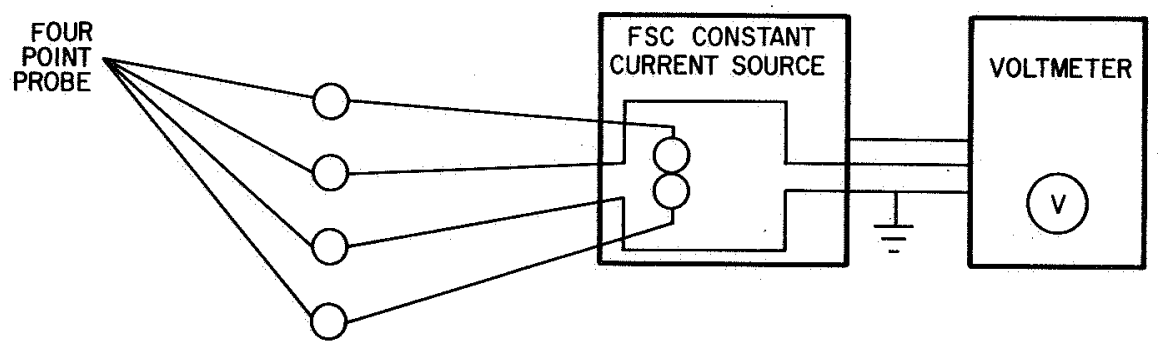

Figure 1. 


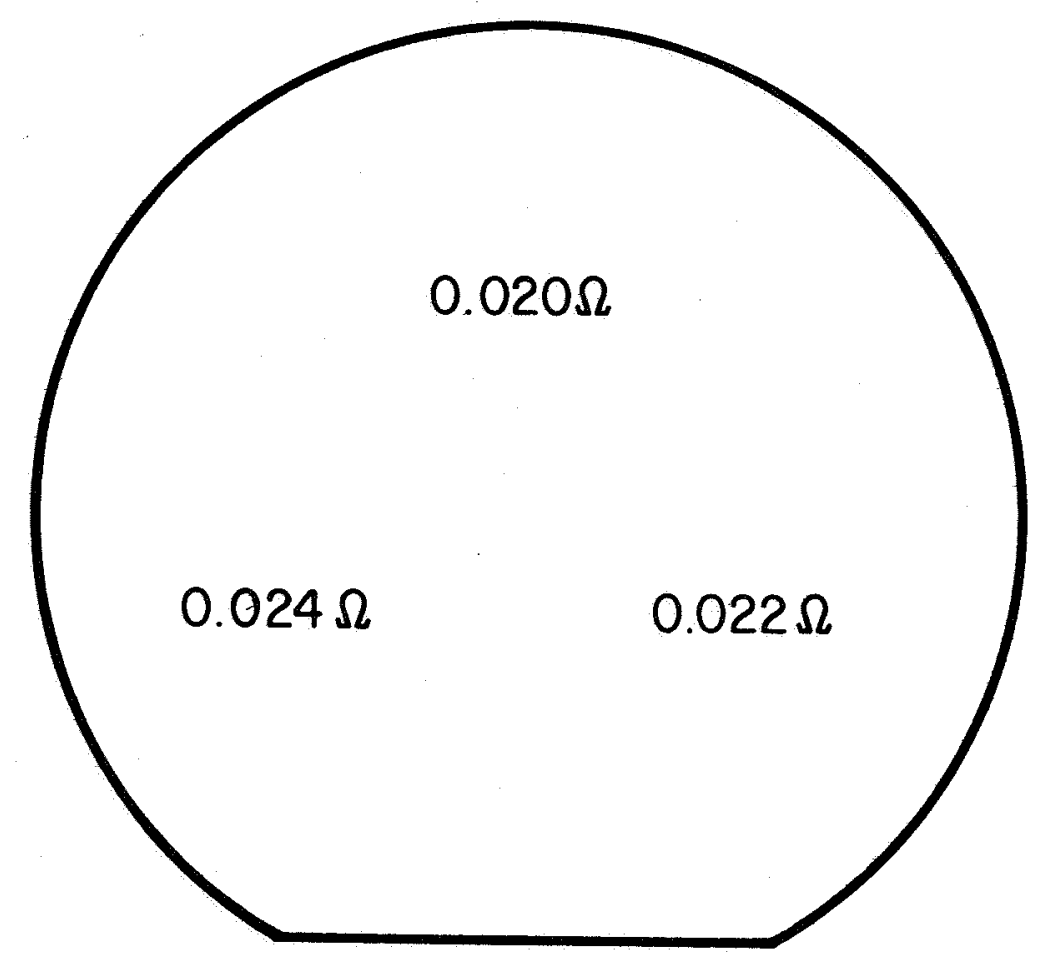

Figure 2. Resistivity Measurement

$$
\begin{aligned}
\frac{V}{I} & =\frac{0.024+0.022+0.020}{3}=0.022 \\
W & =10^{-3} \mathrm{~cm}=250 \mu=2.5 \times 10^{-1} \mathrm{~cm} \\
\rho & =4.53 \mathrm{~W} \frac{\mathrm{V}}{\mathrm{I}} \\
& =4.53 \times 2.5 \times 10^{-1} \times 2.2 \times 10^{-2} \\
& =4.53 \times 2.5 \times 2.2 \times 10^{-3} \\
& =0.25 \Omega-\mathrm{cm}
\end{aligned}
$$




\title{
Method 102 \\ Substrate Orientation \\ (ASTM)
}

1. Purpose

\begin{abstract}
The purpose of this method is to determine the deviation of a slice from the $\langle 111\rangle,\langle 100\rangle$ or $<110>$ planes. It utilizes the reflection of light from a point source which is reflected by etch pits in the wafer to a cross-lined screen. The sample is mounted on a Goniometer calibrated in degrees and minutes to measure angular displacement in two planes.
\end{abstract}

\section{Apparatus}

2. 1 Optical Orientation System Sylvania Crystal Orientation Instrument (or equivalent)

2. 2 Sandblast Unit

S. S. White Industrial Air-Brasive Unit (or equivalent)

3. Materials

3. 1 Sodium Hydroxide, Reagent grade

3. 2 Deionized water

3. 3 Abrasive, S. S. White No. 1 
4. Procedure

4. 1 Sample Preparation

4. 1. 1 Cut a slice according to standard production process.

4. 1.2 Sandblast slice.

4. 1. 3 Etch slice in $10 \%$ sodium hydroxide solution at $90^{\circ} \mathrm{C}$ for about 5 minutes.

4. 1. 4 Rinse thoroughly in deionized water.

4. 1. 5 Blow dry with a nitrogen gun.

\subsection{Measurement}

4.2. 1 Mount slice in sample holder on Goniometer.

4.2.2 Túrn on zirconium arc lamp.

4. 2.3 Adjust Goniometer position to bring the center of the light pattern to a zero reference point on the screen. See Figure 1.

4. 2. 4 The angular reading of the Goniometer adjustment is the degree of misorientation.

4.2. 5 Check measurement by rotating the slice $180^{\circ}$ and noting that deviation is of equal value to 4.2 .4 , but of opposite sign.

\section{Calibration}

5. 1 Set Goniometer to zero position.

5. 2 Substitute first surface mirror for slice in sample holder.

5. 3 Adjust sample holder to place center of light pattern at zero reference point on the cross-lined screen. 
6. Accuracy

Accuracy obtainable is \pm 15 minutes of arc.

7. Test Frequency

Each crystal sliced should be sampled for deviation. 


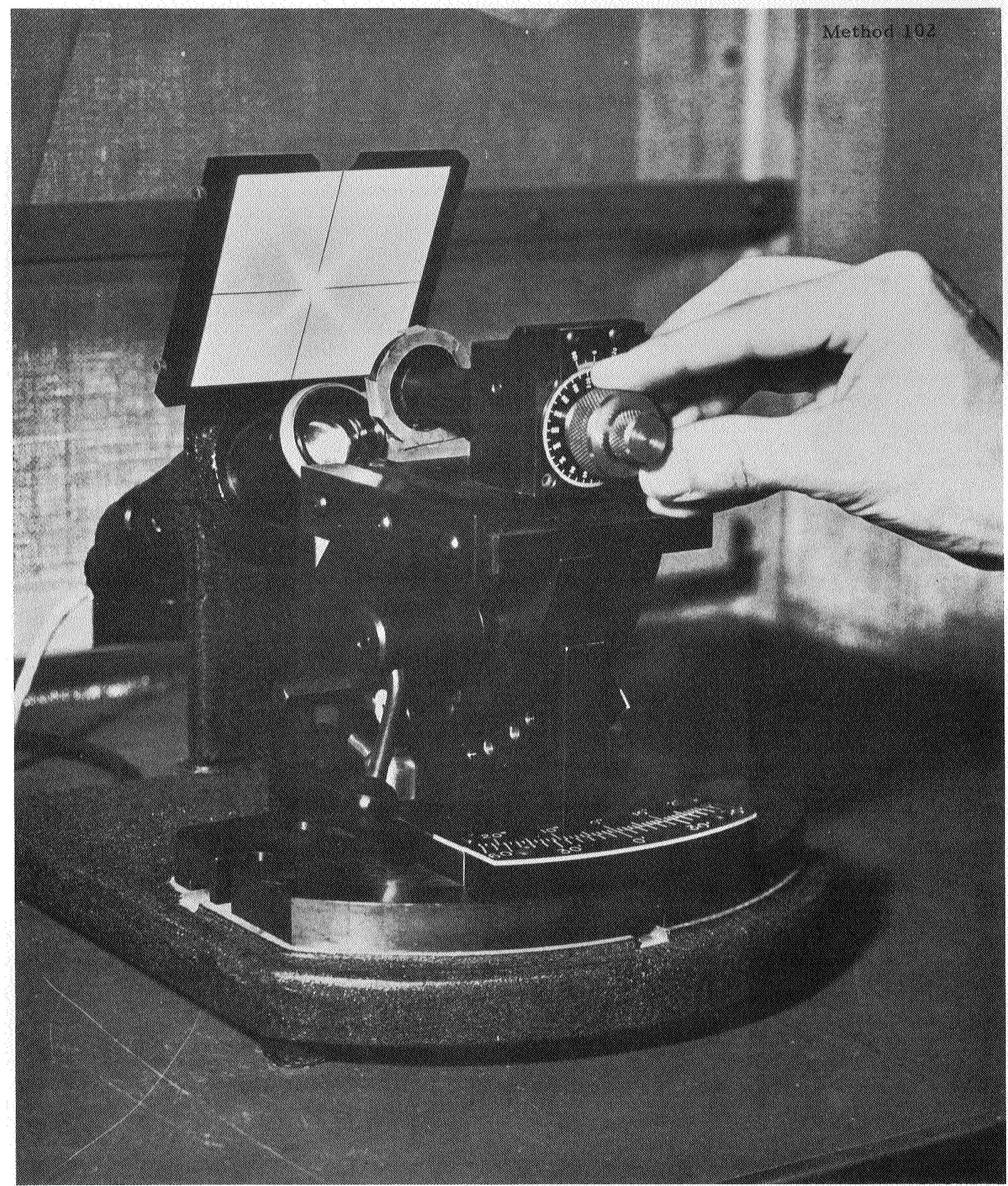

FIGURE I OPTICAL CRYSTAL ORIENTATION INSTRUMENT 
Method 103

Substrate Thickness

1. Purpose The purpose of this test method is to determine the thickness of the substrate by direct measurement.

2. Apparatus

2. 1 Thickness Gauge

Federal Electronic Gauge

Mode1 100B-48

(or equivalent)

2. 2 Calibration Gauges

"Jo Blocks"

(or equivalent)

3. Materials

None.

4. Procedure

4. I Place the substrate, best side up, on the gauge anvil.

4. 2 Depress the foot pedal to lower the probe to the wafer surface.

4. 3 Measure the wafer thickness in the five locations shown in Figure 1.

4. 4 Compare the thickness readings and determine average thickness and range of variation.

\section{Calibration}

Measure a thickness standard of known size and, using the zero control, adjust the meter until there is direct correlation. 


\section{Accuracy}

Accuracy obtainable is \pm 1 micron.

\section{Test Frequency}

This should be a periodic test. 
Method 103

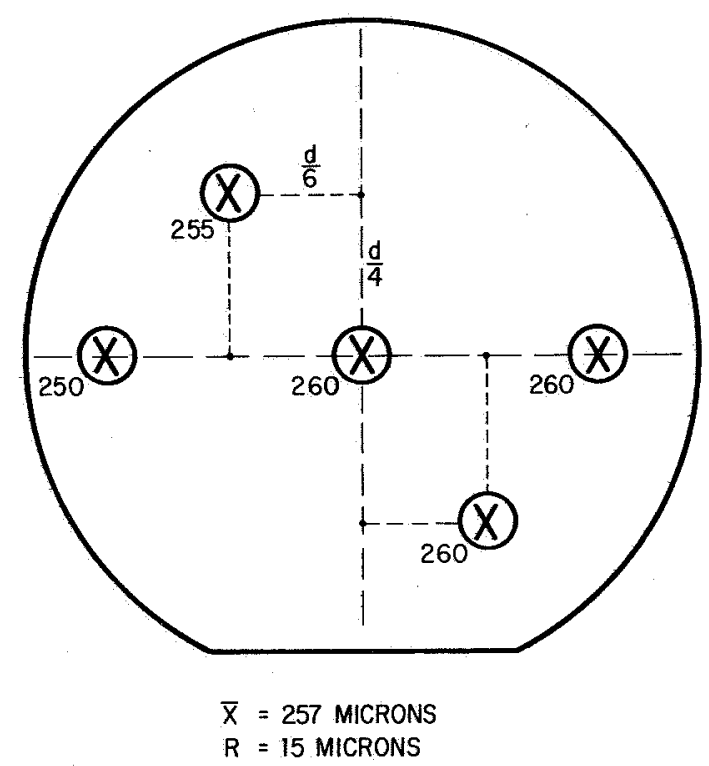

Figure 1. Thickness Measurement 
Method 104

Substrate Parallelism

1. Purpose The purpose of this test is to measure the degree of parallelism of the substrate sides. Thickness measurement across the substrate is compared to determine any taper.

2. Apparatus

2. 1 Thickness Gauge

Federal Electronic Gauge

Mode1 100B-48

(or equivalent)

2. 2 Calibration Gauges

"Jo Blocks"

(or equivalent)

3. Materials

None.

4. Procedure

4. 1 Place the substrate, best side up, on the anvil. Figure 1.

4. 2 Depress the foot pedal to lower the probe to the wafer surface.

4. 3 Note the thickness of the substrate as indicated on the meter dial.

4. 4 Repeat 4.2 and 4.3 several times and obtain an average across the substrate if classification is necessary.

\section{Calibration}

Measure a thickness standard of known size and, using the zero control, adjust the meter until there is direct correlation. 
6. Accuracy

Accuracy obtainable is \pm 1 micron.

7. Test Frequency

Each substrate should be measured. 


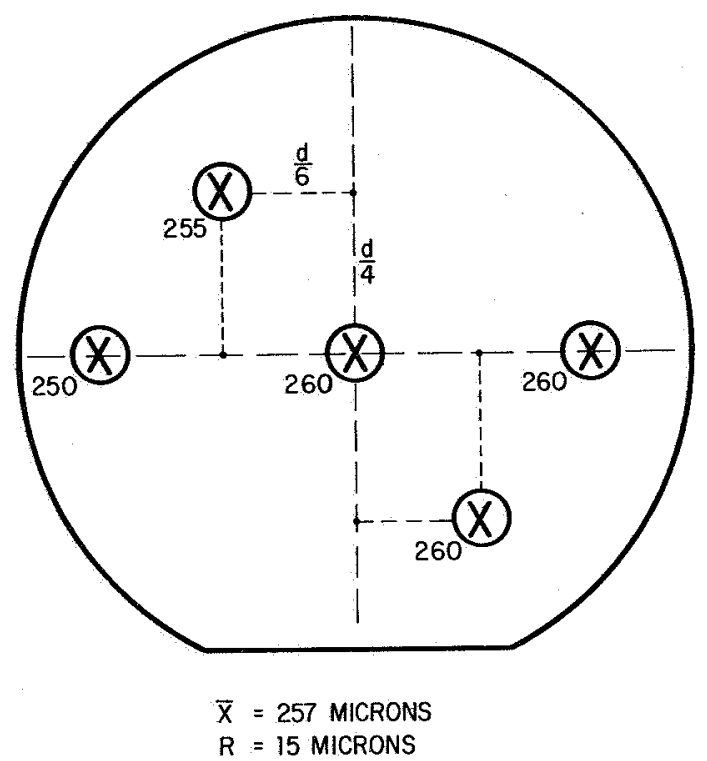

Figure 1. Thickness Measurement 
Method 105

Substrate Flatness

1. Purpose This test may be used to determine bowing of the substrate. It utilizes the flexing of a bowed substrate during thickness measurement.

2. Apparatus

2. 1 Thickness Gauge (contact type)

Federal Electronic Gauge

(or equivalent)

2. 2 Microscope Lamp

American Optical Co.

Model A0653

(or equivalent)

3. Materials

None.

4. $\quad$ Procedure

4. 1 Adjust lamp to provide low angle (less than $45^{\circ}$ ) illumination of the wafer upon the gauge anvil.

4. 2 Lower the gauge probe to the wafer.

4. 3 Observe wafer surface for deformation (convex bow) or raising of opposite side (concave bow). Figure 1.

4. 4 Repeat 4.3 to include center and three perimeter locations.

5. Calibration

None.

6. Accuracy

Accuracy is dependent upon the thickness of the substrate and its surface finish. 


\section{Test Frequency}

This test should be performed on all thin (less than 100 micron) substrates and periodically on others.

NOTE: This test may be performed concurrently with thickness or parallelism measurements. 

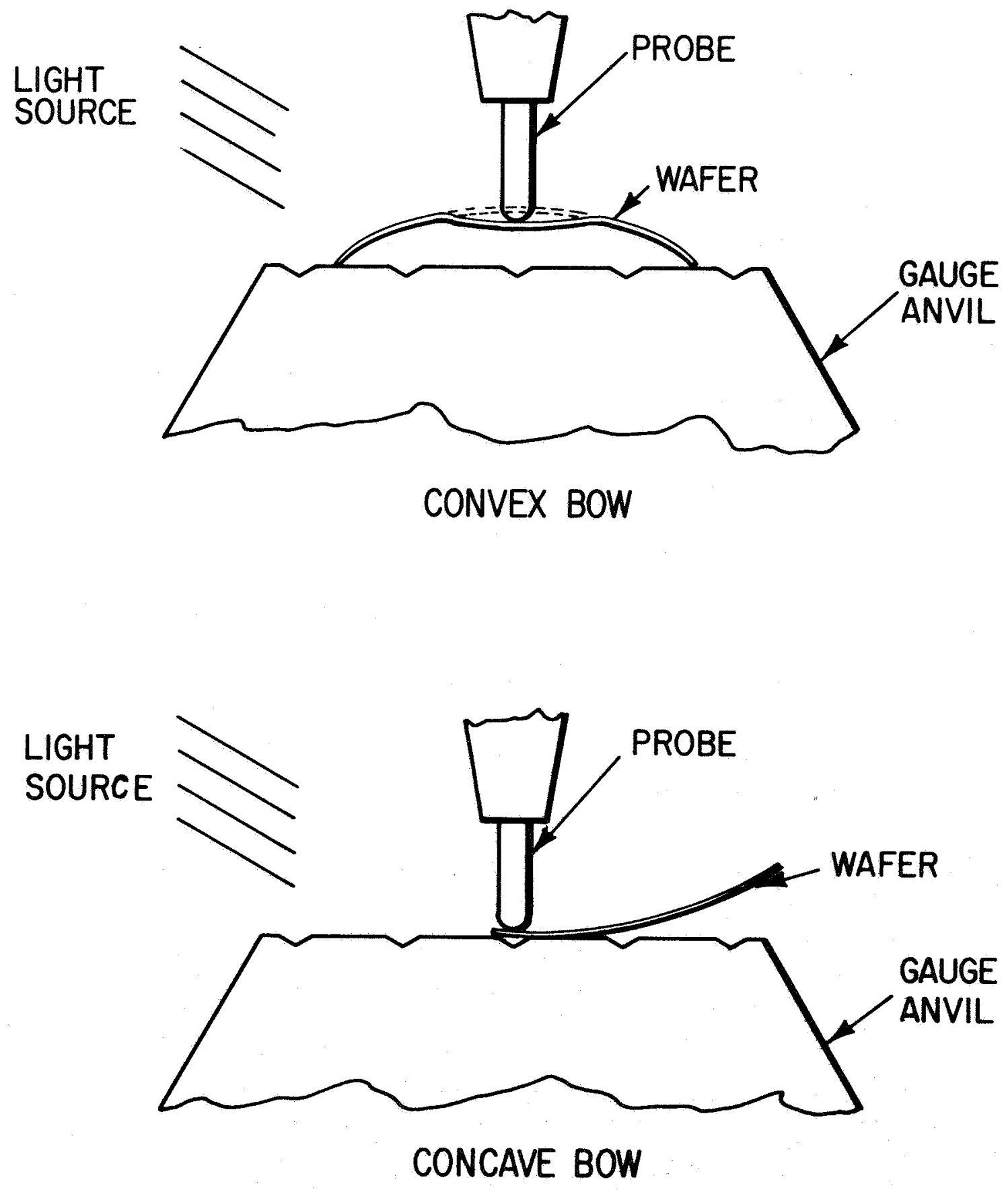

Figure 1. Substrate Bowing 
Method 106

Substrate Crystal Perfection

(Dow Corning, ASTM)

1. Purpose The purpose of this method is to determine crystalline perfection of substrates. It utilizes a peferential etch to reveal dislocations, lineage, slip, etc.

2. Apparatus

2. 1 Metallurgical Microscope

Bausch and Lomb, Model 31-20-68-38 (DMETR)

(or equivalent)

3. Materials

3. 1 Chromium Trioxide, Reagent grade.

3. 2 Hydrofluoric Acid, Electronic grade.

3. 3 Deionized Water.

4. Procedure

4. 1 Preparation

4.1.1 Obtain a polished substrate.

4.1.2 Mix two stock solutions:

$$
\begin{aligned}
& \text { A - } 100 \mathrm{ml} \text { Deionized Wate } \mathrm{r} \\
& 50 \text { grams Chromium Trioxide } \\
& \text { B - } \quad 50 \mathrm{ml} \mathrm{Hydrofluoric} \mathrm{Acid}
\end{aligned}
$$

NOTE: Solution may be stored after mixing. 
4. 1.3 Etch wafers for $10-12$ minutes $\left(25^{\circ} \mathrm{C}\right)$.

4. 1. 4 Rinse thoroughly with deionized water.

4. 1.5 Dry with nitrogen gun.

\section{2 Inspection}

4.2. 1 Using a microscope with a grid eyepiece, count the number dislocations in one or more squares of the grid at each of nine locations on the sample. See Figures 1 and 2.

4.2. 2 Calculate the dislocation density as pits per square centimeter using the following equation:

$$
\text { Density }=\frac{\text { Total No. Pits }}{\text { No. Squares } \times \text { Area of One Square }}
$$

4.2.3 Note the number found of each of the following defects:

\subsubsection{Lineage A plane imperfection appearing} as a linear array of etch pits with density greater than 25 per millimeter. See Figure 3. Pits are aligned point to base.

4.2.3.2 Gross Lineage Parallel lineages within 200 microns of one another.

4.2.3.3 Slip Line A plane imperfection in a crystal appearing as a very straight line of pits with density greater than 25 per millimeter. Pits will be aligned with bases on a straight line. See Figure 4.

4.2.3.4 Grain Boundary The boundary between two areas of different crystal orientation. Etch pits cannot be resolved at boundary. See Figure 5.

4. 2. 3. 5 Twin Lamella A boundary with no obvious change in orientation across the boundary (found only on < $111>$ planes). See Figure 6. 
5. Calibration

None.

6. Accuracy

Not applicable.

\section{Test Frequency}

This test should be performed periodically. 
Method 106

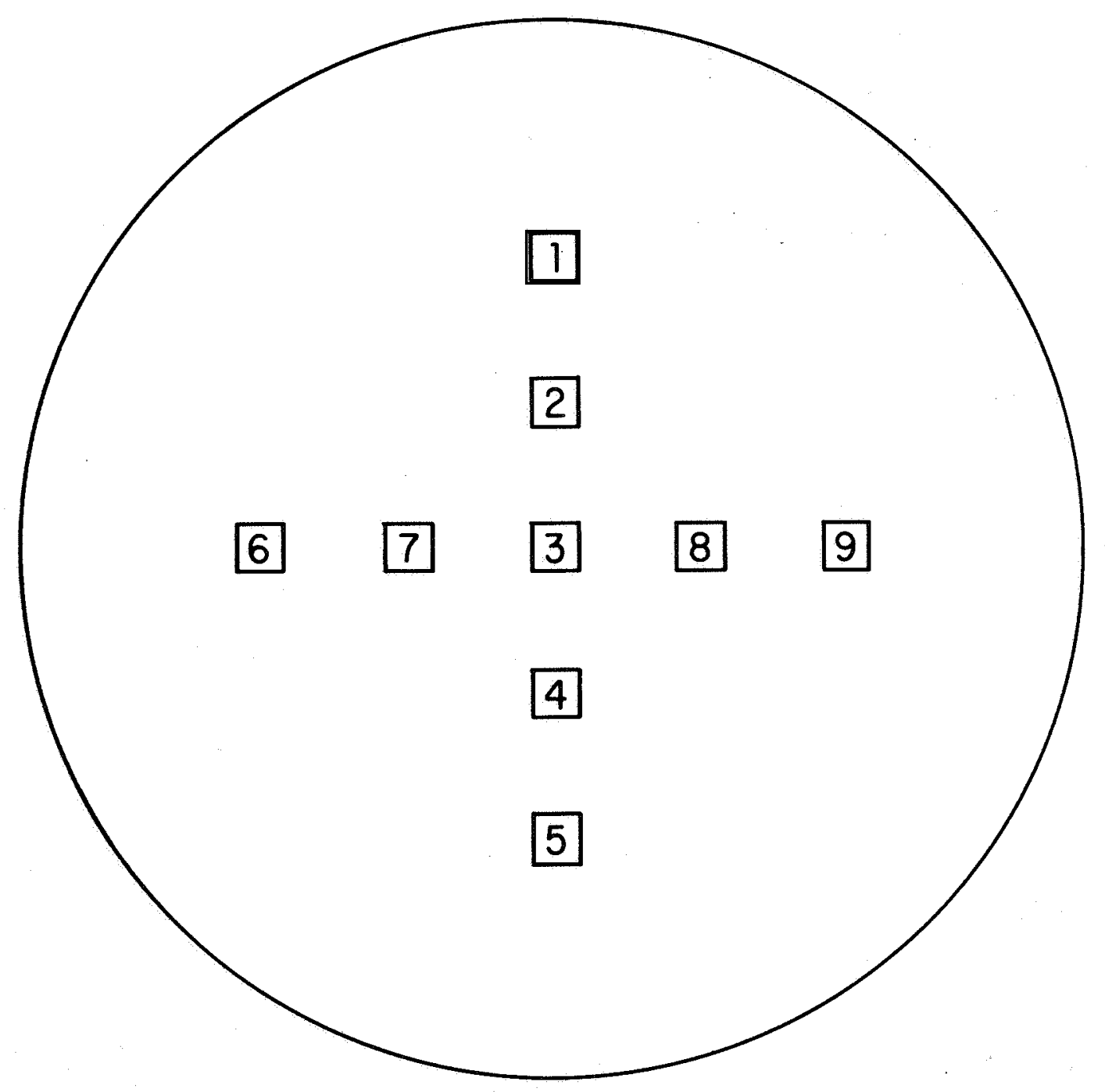

Figure 1. Sampling Pattern 
Method 106
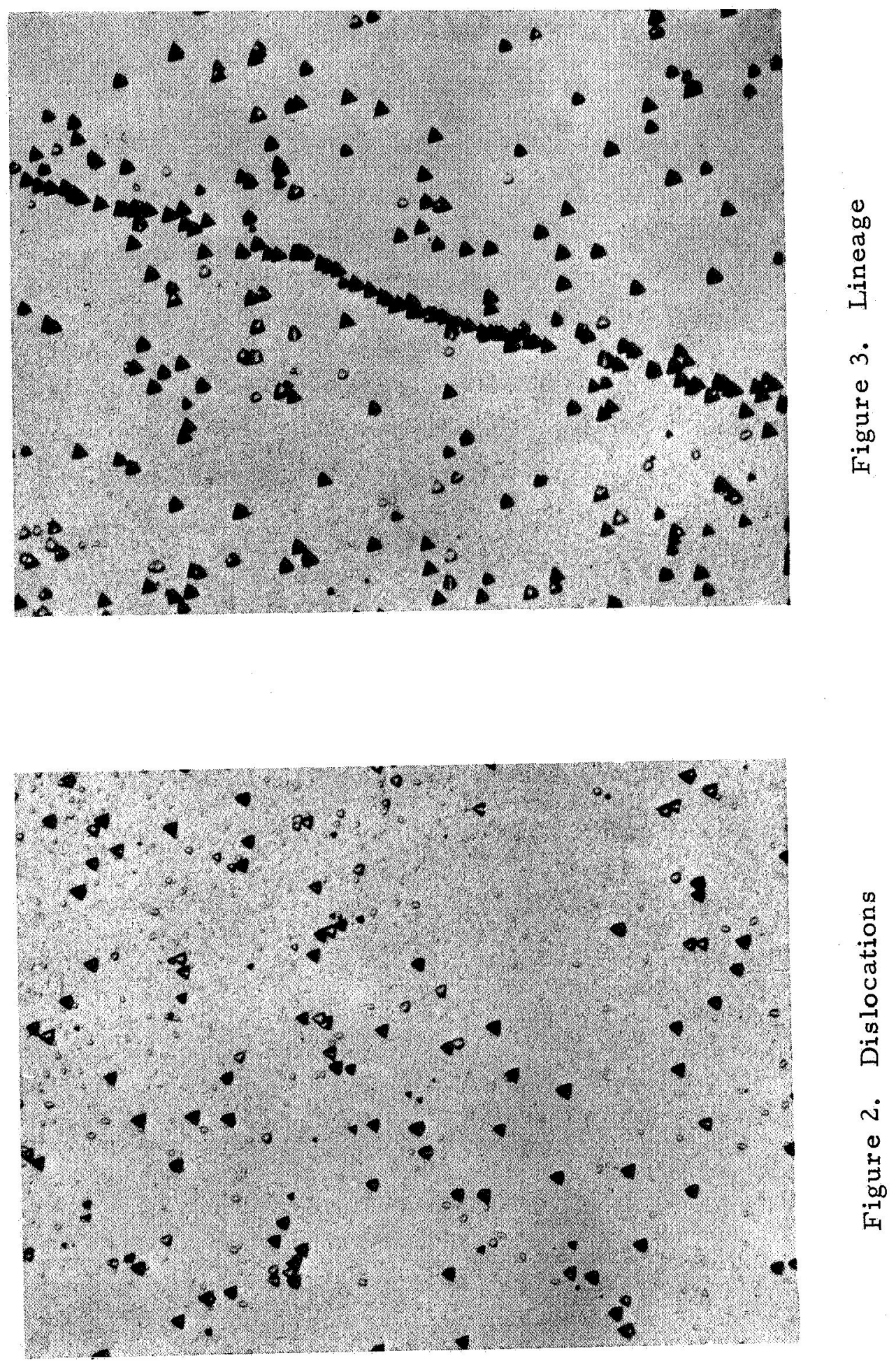
Method 106

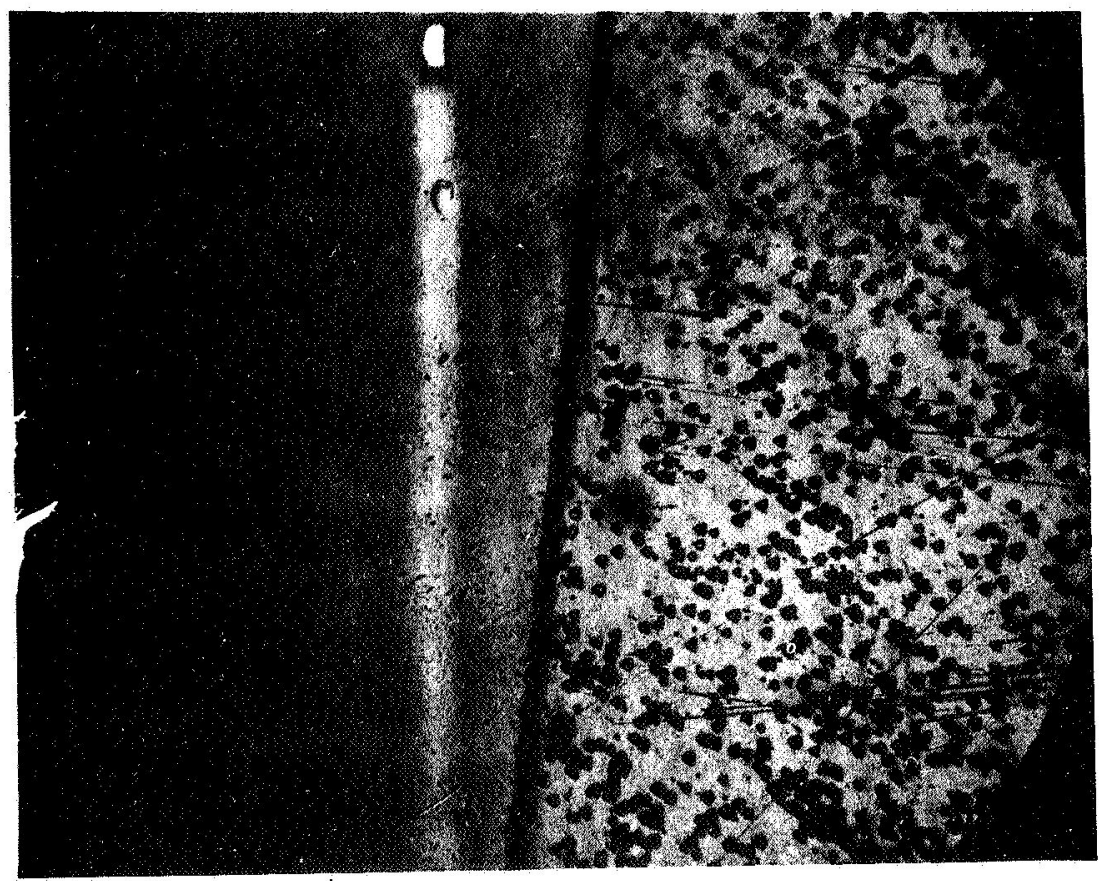

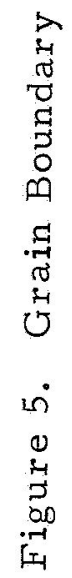

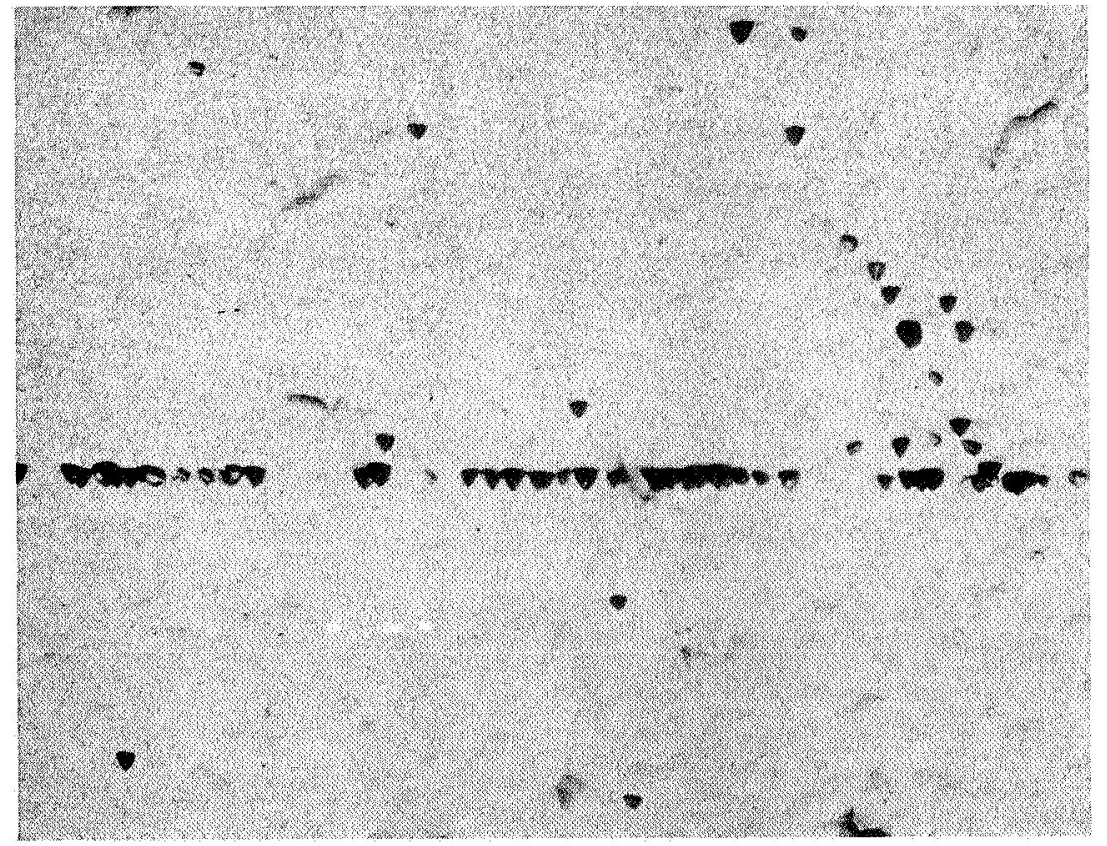

$\stackrel{0}{\stackrel{1}{7}}$

究

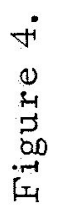




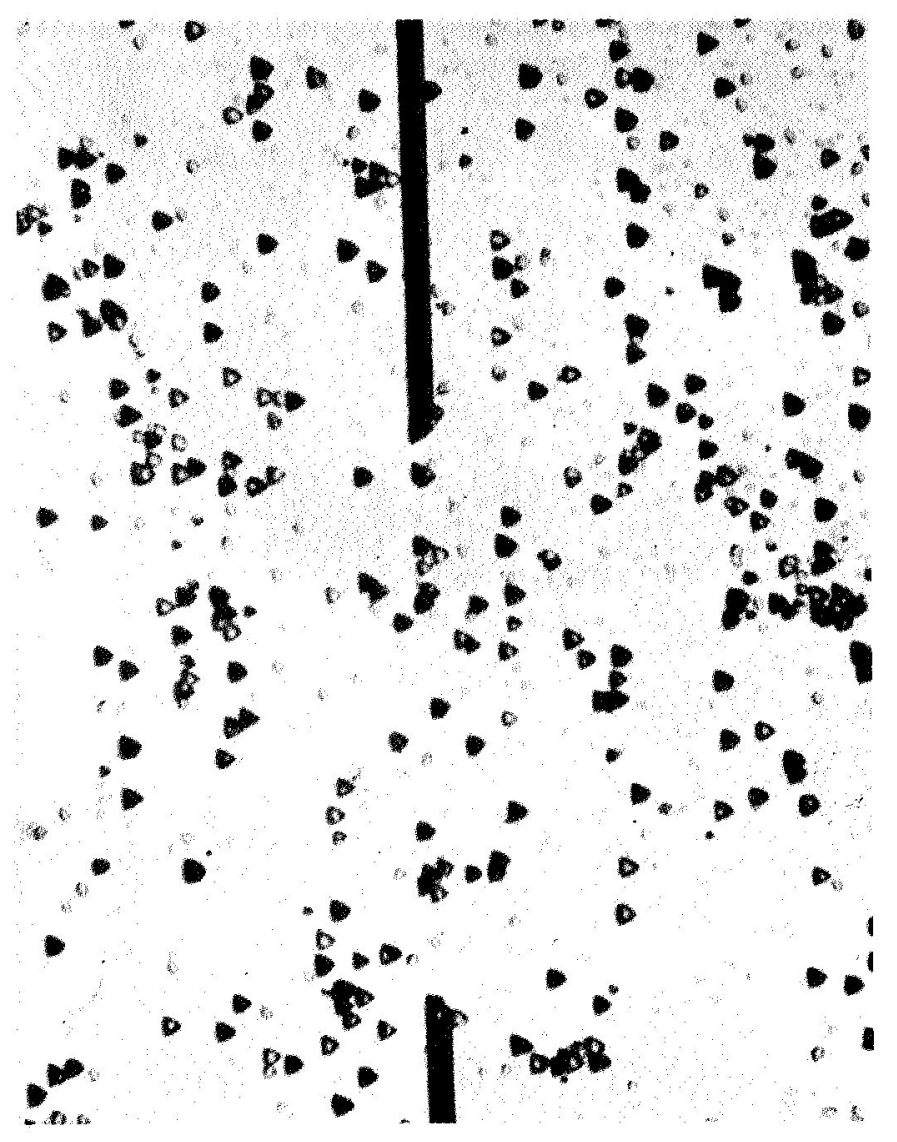

Figure 6. Twin Lamella 


\author{
Method 107 \\ Substrate Conductivity Type \\ (Hot-Probe)
}

1. Purpose This test may be used to determine the conductivity type of the device substrate. It utilizes the thermoelectric effect where the majority carriers tend to migrate to the hot probe, causing deflection of a galvanometer.

2. Apparatus

\title{
2. 1 Galvanometer
}

(any source)

2. 2 Soldering Iron, small

(any source)

3. Materials

None.

4. Procedure

4. 1 Connect the equipment as shown in Figure 1.

4. 2 Turn on the soldering iron and allow 5 minutes to reach temperature.

4. 3 Place the substrate to be tested upon the aluminum.

4. 4 Touch the sample surface with the probe tip.

4. 5 Observe the Galvanometer deflection to determine conductivity type.

\section{Calibration}

At the start of testing a small battery may be placed on the aluminum strip and probed to verify correct connections. 
6. Accuracy

This test is $100 \%$ accurate below $5 \Omega-\mathrm{cm}$ resistivity material.

7. Test Frequency

This test should be performed on a sample basis on each lot of wafers. 


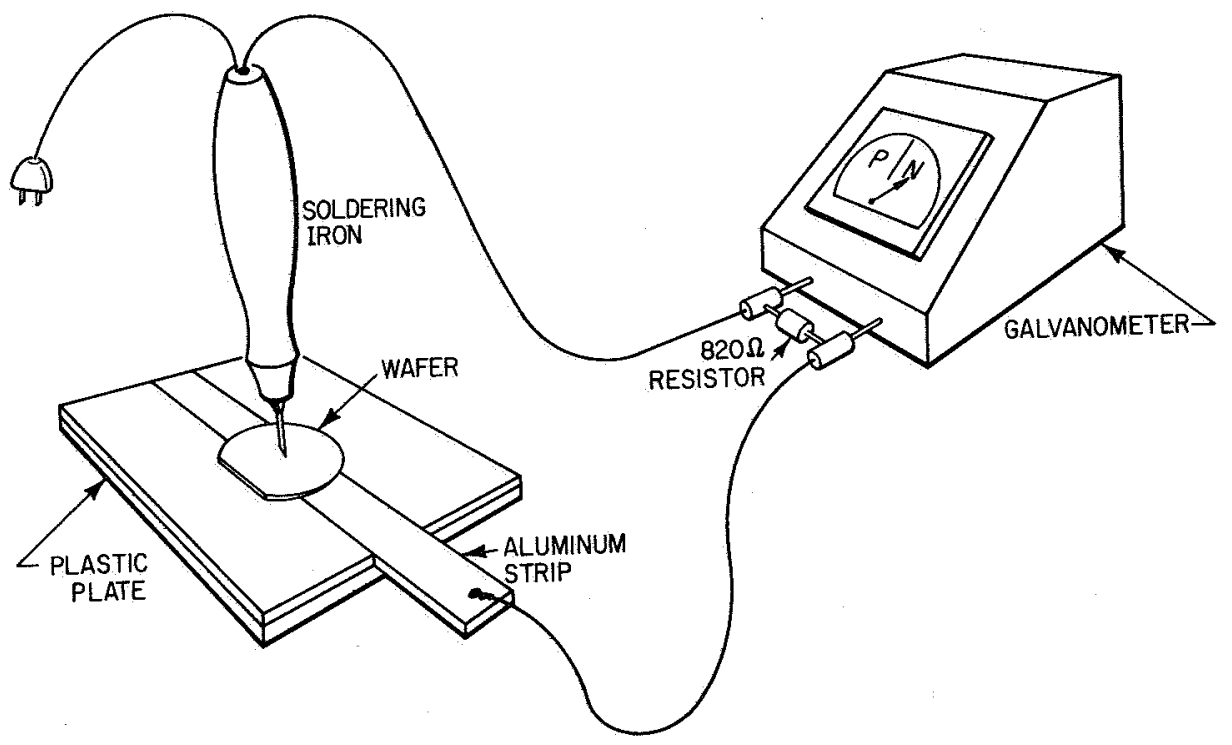

Figure 1. Hot Probe Setup 
Method 108

Substrate Surface Finish

1. Purpose The purpose of this test is to determine the surface finish of the polished substrate.

2. Apparatus

2. 1 Microscope Illuminator

American Optical AO No. 653

(or equivalent)

3. Materials

None.

4. Procedure

4. 1 Pick up wafer with clean tweezers.

4. 2 With the light source collimated, rotate the polished surface so that it is illuminated through a wide range of angles.

4.3 Note any roughness, ripple or "orange-peel".

5. Calibration

Not applicable. 
6. Accuracy

Surface irregularities with a peak-to-valley distance of about $2900 \AA$ ( $\frac{\lambda}{2}$ for sodium) may be observed.

7. Test Frequency

All wafers should be inspected. 


\author{
Method 109 \\ Substrate Width or Diameter
}

1. Purpose This method may be used to measure the width or diameter of a substrate to determine process compatibility.

2. Apparatus

2. 1 Steel Rule, six inch (any source)

2. 2 Go-No-Go Gauge (any source)

3. Materials

3. 1 Bibulous Paper

4. Procedure

4. 1 Direct Measurement

4. 1. 1 Place steel rule on a clean sheet of paper.

4. 1. 2 Place wafer upon rule with maximum dimension along ruled edge.

4. 1.3 Note width or diameter as required.

4. 2 Go-No-Go

4.2.1 Place two sheets of bibulous paper upon the working surface. 
4. 2. 2 Upon one place a $4^{\prime \prime} \times 4^{\prime \prime}$ thin metal plate containing a hole of maximum allowable diameter.

4. 2. 3 Upon the other, place a plate with a minimum diameter hole.

4.2. 4 Gauge wafers to be smaller than maximum and greater than minimum diameters.

\section{Calibration}

None.

6. Accuracy

6. 1 Accuracy obtainable is $\pm 1 / 32$ inch.

6. 2 Accuracy required is $\pm 1 / 16$ inch.

\section{Test Frequency}

7. 1 Direct measurement should be done periodically.

7. 2 Go-No-Go testing should be performed on all wafers to be heat treated at high temperatures. 


\author{
Method 110 \\ Water Resistivity
}

1. Purpose This method may be used to determine the resistivity of high purity water. It utilizes a resistance bridge with temperature compensation.

2. Apparatus

2. 1 Resistance Bridge

Beckman Solu Bridge, Model RD-146-Y1

(or equivalent)

3. Materials

None.

4. Procedure

4. 1 Plug instrument power cord to 110 volt source.

4. 2 Place instrument on table or other solid footing.

4. 3 Connect cell to binding posts 1 and 2 (no polarity).

4. 4 Attach cell to water outlet.

4. 5 Turn water to about one-fourth full flow.

4. 6 After one to two minutes, measure water temperature at discharge.

4. 7 Adjust temperature compensation dial to agree with water conditions. 
4. 8 Using sensitivity dial, adjust tuning-eye (iris) to best close distune (two faint shadows will form one matched dark one).

4. 9 Allow one minute to stabilize, and note resistivity indicated on sensitivity dial.

\section{Calibration}

Calibrate per manufacturer's instructions.

6. Accuracy

Accuracy obtainable is $\pm 10 \%$.

\section{Test Frequency}

This test should be performed periodically. 


\author{
Method 111 \\ Water Solids \\ (Particle Count)
}

1. Purpose This method may be used to determine the number of undissolved particles present in high purity water.

2. Apparatus

2. 1 Microscope

Bausch and Lomb, Model 31-20-68-38 (DMETR)

(or equivalent)

3. Material

3. 1 Small Knife

Exacto

(or equivalent)

3. 2 Tweezers

EREM No. 2A

(or equivalent)

3. 3 Filter Paper, 0.45 micron membrane

Gelman, GA-46

(or equivalent)

4. Procedure

4. 1 Obtain a $0.45 \mu$ membrane filter from any area utilizing these filters within the plant. Record time, date, area, department and amount of water flushed through filter. Label direction in which water flowed through filter. 
4. 2 With an exacto knife, cut about a quarter (1/4) inch area from the center of membrane filter.

4. 3 With tweezers, pick the center piece and place face up (upstream) under the prescribed microscope and count particles under a $40 \mathrm{X}$ magnification. In order to bring lens into focal adjustment, focus on edge of filter paper with coarse adjustment knob of microscope then bring particles into focus with fine adjustment knob. All distinguishable particles shall be counted as one.

4. 4 Divide the number of particles counted by the number of liters flushed through filter and multiply by $8 \times 10^{5}$. If no particles are seen in the first field of view, scan a total of fifteen (15) fields of view, divide number by fifteen (15) and proceed with calculations.

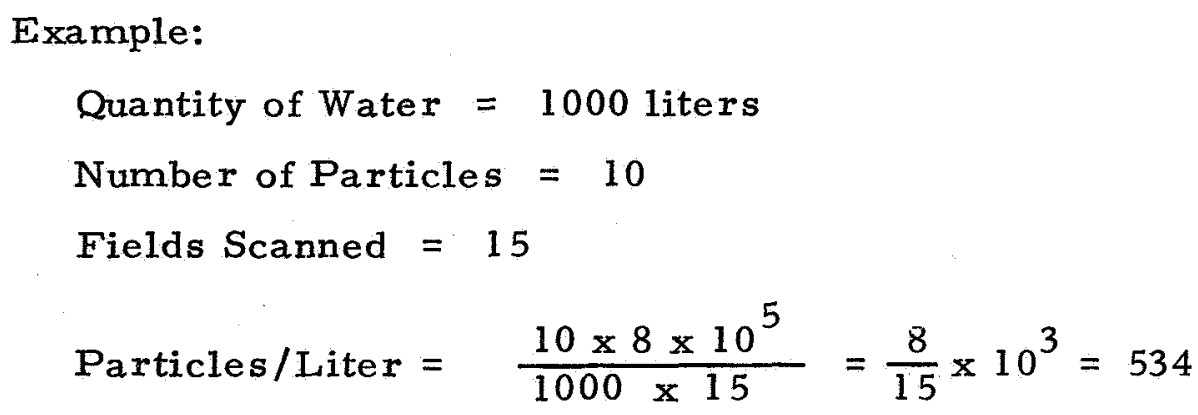

\section{Calibration}

None.

6. Accuracy

Accuracy obtainable is $\pm 10 \%$ for 0.5 micron particles.

7. Test Frequency

This test should be performed periodically. 


\section{Method 112 \\ Water Bacteria Count}

1. Purpose This test may be used to obtain a bacteria count for high purity water.

2. Apparatus

2.1 Bacteria Counter

Quebec Colony Counter

(or equivalent)

3. Materials

3. 1 Pipette, $1 \mathrm{ml}$

3. 2 Petri Dish, $100 \times 15 \mathrm{~mm}$

3. 3 Broth Solution, Agar

4. Procedure

\section{1 Preparation of Broth}

4.1.1. Weigh out 4.8 grams of Agar and mix in a clean $400 \mathrm{ml}$ beaker with $200 \mathrm{ml}$ of cold distilled

D. I. Water $\left(25^{\circ} \mathrm{C}\right)$.

4. 1. 2 Place mixture on a hot plate and heat to boiling to dissolve the medium completely. Keep well stirred.

4. 1. 3 Cover container with aluminum foil and place in autoclave. 


\section{2 Sterilization}

4.2. 1 Petri dishes, $1 \mathrm{ml}$ pipette, water sample bottles and glass stoppers and Agar. Nutrient medium shall be sterilized at 15 pounds pressure for 15 minutes. Wrap Petri dishes individually in aluminum foil.

\section{3 Actual Procedure}

4.3. 1 Allow water to run for at least five minutes prior to drawing samples. Draw water sample in sterile container, fill to top, stopper, wrap top of bottle in tin foil and identify.

4.3.2 The sample bottle should be shaken vigorously 25 times, and $1 \mathrm{ml}$. should be drawn with a standard sterile pipette. If a second sample is desired, shake the sample bottle vigorously a nother 25 times before the second is withdrawn.

4.3. 3 The withdrawn $1 \mathrm{ml}$ water sample should be transferred to a sterilized $100 \times 15 \mathrm{~mm}$ petri dish. Add $10 \mathrm{ml}$ of liquefied Agar (which is maintained at a $44^{\circ} \mathrm{C} \pm 1^{\circ}$ ) directly to the petri dish. The cover of the petri dish is lifted just enough for the introduction of either the pipette or culture medium. The lips of all test tubes or flasks used for pouring, the medium should be flamed. The medium and sample in the petri dish should be thoroughly mixed and uniformly spread over the bottom of the petri dish by tilting and rotating the dish.

4.3.4 Allow the Agar medium and water sample to solidify. The medium in all plates should be solidified as rapidly as possible after pouring and the plates placed immediately in the appropriate incubator. Not more than 20 minutes should elapse between pouring and plating.

4.3. 5 Invert the petri dish and store in incubator at $20^{\circ}-25^{\circ} \mathrm{C}$ for 72 hours \pm 3 hours. 
4.3.6 Counting should be done with a Quebec Colony Counter. If such equipment is not available, counting may be done with a lens giving magnification of 1.5 diameters. In order to insure uniformity of counting conditions, illumination equivalent to that provided by the Quebec Colony Counter should be employed.

4.3.7 Counting Colonies: At a 0 - 1. $5 \mathrm{X}$ magnification, count total number of colonies in 9 squares, and calculate number of colonies per $\mathrm{ml}$ of water. See Figure 1.

5. Calibration

None.

6. Accuracy

Accuracy obtainable is $\pm 5 \%$.

7. Test Frequency

Test should be performed periodically. 
Method 112

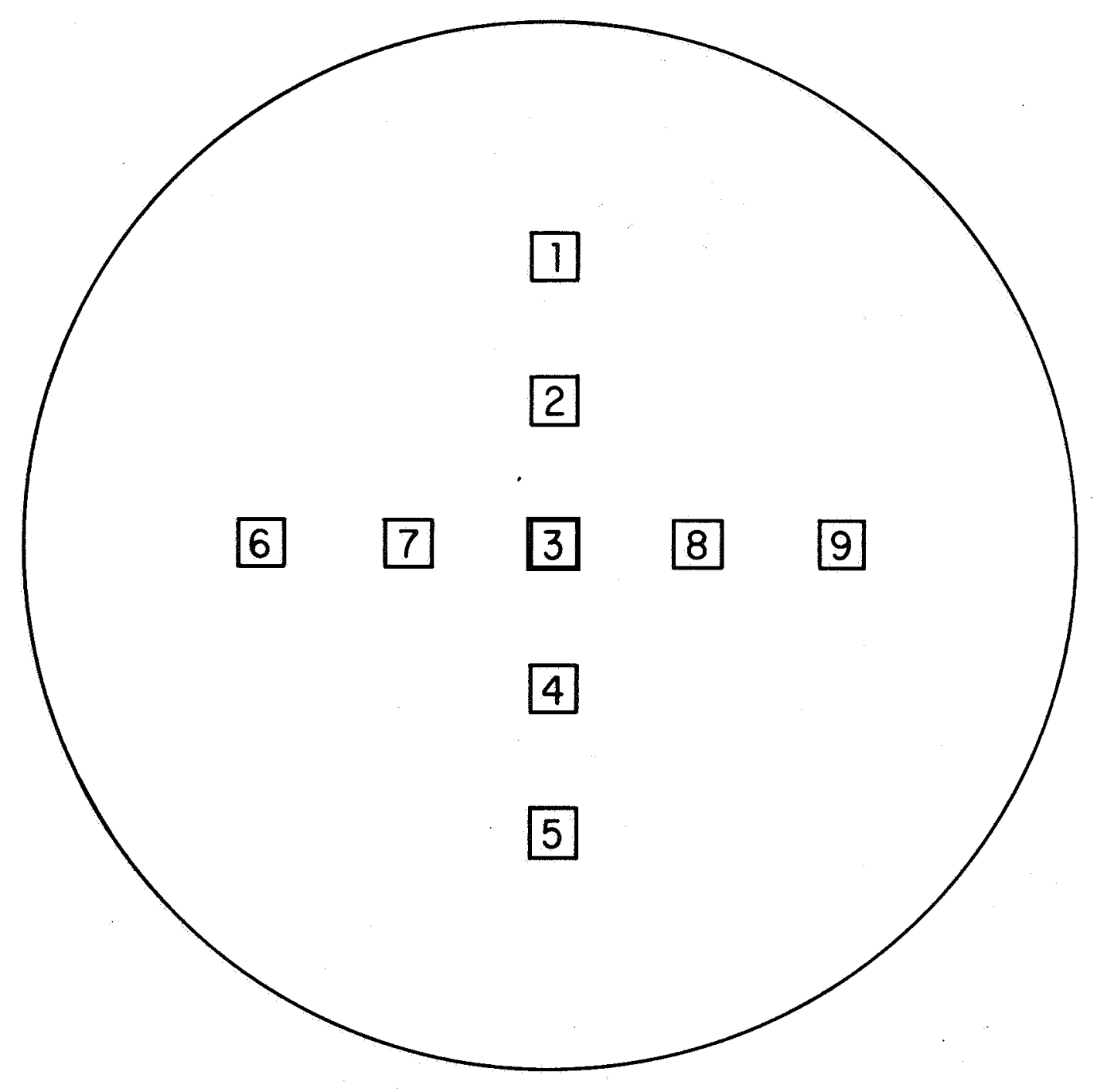

Figure 1. Sampling Pattern 
Method 113

Photoresist Viscosity

(ASTM No. D445-65)

1. Purpose This method may be used to determine the viscosity of any photoresist. It utilizes a Cannon-Fenske Viscometer where a fixed volume of the liquid is allowed to flow by gravity through a capillary.

2. Apparatus

2. 1 Viscometer

Cannon-Fenske Viscometer

Series 200, 300 and 500

(any source)

2. 2 Viscometer Holder

(any source)

2. 3 Viscometer Thermostat

(any source)

2. 4 Thermomete $\mathrm{r}$

Kinematic Viscosity Test Thermometer

(any source)

2. 5 Timer 0.2 second or less/Division

(any source)

3. Materials

3. 1 Photoresist

3. 2 Photoresist Stripper

3. 3 Viscosity Oil Standard 


\section{Procedure}

4. 1 Select a clean viscometer which will give the minimum specified time with the given photoresist or 200 seconds, whichever is greater. See Table I.

4. 2 Charge the viscometer with the photoresist to be evaluated.

4. 3 Allow the charged viscometer to remain in the bath long enough to reach $24 \pm 1^{\circ} \mathrm{C}$.

4. 4 Use pressure to adjust the head level of the photoresist sample to a position about $5 \mathrm{~mm}$ ahead of the first timing mark.

4. 5 Measure to the nearest 0.2 second, the time required for the meniscus to pass from the first timing mark to the second.

4. 6 Calculate the kinematic viscosity as follows:

Kinematic Viscosity $=\mathrm{Ct}$

where: $\quad \mathrm{C}=$ Calibration constant of the viscometer $t=$ Flow Time

5. Calibration

Calibrate the viscometer with any standard oil, by measuring its flow time and applying the following equation:

$$
C=\frac{v}{t}
$$

where: $V=V$ iscosity for the standard oil.

\section{Accuracy}

Accuracy obtainable is $\pm 1 \%$.

\section{Test Frequency}

This test should be performed for every lot of photoresist. 
Viscometer Applicable for Various Photoresists

\begin{tabular}{|l|c|}
\hline Resist & Series \\
\hline KPR & 200 \\
KPR -2 & 200 \\
KOR & 300 \\
KMER & 500 \\
KTFR & 500 \\
\hline
\end{tabular}

Table I 
Method 114

Photoresist Viscosity

(Torque)

1. Purpose This test may be used as a rapid method for determining the viscosity of raw KMER. It utilizes the resistance the fluid has to the motion of a rotating spindle. It may be adapted for other types of photoresist, if a correlation study is made with a Cannon-Fenske Viscometer per ASTM No. D445-65.

2. Apparatus

2. 1 Synchro-Lectric Viscometer, Brookfield RVT model.

Scientific Products

(or equivalent) See Figure 1 .

2. 2 Beakers, pyrex, 100, 600 and 1000 milliliter. (any source)

2. 3 Stirrer, Utility single speed No. 58000.

Scientific Products Company, (or equivalent)

3. Materials

\section{1 KMER}

\section{2 KMER thinner}

3. 3 Trichloroethylene

3. 4 Acetone

4. Procedure

\section{1 Preparation}

4. 1. 1 Using a sink with a vented hood, pour 50-100 $\mathrm{ml}$ of trichloroethylene into a $600 \mathrm{ml}$ beaker and rotate to ensure walls of beaker are cleaned. Pour TCE down the drain. 
4. 1. 2 Pour $50-100 \mathrm{ml}$ of acetone into beake $\mathrm{r}$ and rotate to ensure all TCE is absorbed and pour down the drain. Blow dry with $\mathrm{N}_{2}$ gun.

4. 1.3 Into DRY beaker, pour 350-400 $\mathrm{ml}$ of raw KMER (shake bottle vigorously for 30 seconds immediately before use). Set timer for 10 minutes and stir KMER with electric stirrer. Into a 100-ml beaker pour 60-70 $\mathrm{ml}$ of KMER thinner. When ten minutes are up, stop electric stirrer, dismantle blade and place into beaker full of thinner.

\section{2 Measurement}

Note: Room temperature shall be $24 \pm 1^{\circ} \mathrm{C}$.

4.2. 1 Bring the sample directly under the spindle holder, install spindle No. 2 (number is on neck of shaft) and make sure that the spindle is suitable centered. The spindle should be correctly immersed to the groove found on the shaft. Do this by lowering or raising entire spindle assembly on stand. NOTE: Handle spindle with forefinger and thumb. Grasp shaft between groove and spindle only.

4.2. 2 Turn speed selector knob to 50. With right hand turn the on-off toggle switch to on and allow spindle to rotate at least a dozen times to equilibrate itself with the viscosity of the material. With left hand press down on clutch lever in back of spindle assembly. This will automatically "lock" dial. Turn toggle switch off and record dial reading. Important: If dial reading does not come into scale view, do not release clutch lever. Simply turn toggle switch on and off until dial comes into view. Release clutch lever and record reading.

4.2. 3 Record dial reading on to proper $\log$ sheet and from Brookfield Factor Finder obtain proper multiplication factor. This is done in the following manner:

4.2.3.1 The top two letters on the "slide" designate the viscometer model number. Slide the rule until letters RV appear. 
4. 2.3.2 The number in large bond type directly below the model letters designates the spindle number. Slide the rule to No. 2 .

4.2.3.3 The numbers on the left side of the column designates the speed of the spindle. Go down to 50 .

4.2.3. 4 The numbers on the right side of the column designates multiplication factor. The number directly across the applied speed is the proper multiplication factor. Multiply dial reading by multiplication factor and enter results in proper log sheet. Viscosity has been measured in centipoise.

Example:

$\begin{gathered}\text { Spindle } \\ \text { No. }\end{gathered}$
2 $\frac{\text { Speed }}{50} \frac{\begin{array}{l}\text { Dial } \\ \text { Reading }\end{array}}{61} \frac{\begin{array}{c}\text { Multiplication } \\ \text { Factor }\end{array}}{8} \frac{\text { Viscosity }}{488 \mathrm{cp}}$

4.2. 4 Raise entire spindle unit out of the sample and remove spindle. Place dirty spindle into beaker full of KMER thinner. Cover sample in beaker with tin foil and do not remove until sample is ready to be checked for surface tension.

\section{Calibration}

5. 1 Make sure that the Brookfield Viscometer is perfectly level; if not, adjust by turning the knobs on the stand until the bubble level indicates instrument is level.

5. 2 Spindle size and speed calibration may be checked by correlation with a Cannon-Fenske Viscometer, series 500, per Method 113, "Photoresist Viscosity" (Cannon-Fenske).

6. Accuracy

Obtainable accuracy with this procedure is \pm 1 percent.

7. Test Frequency

This test should be performed on a sample of each photoresist lot. 

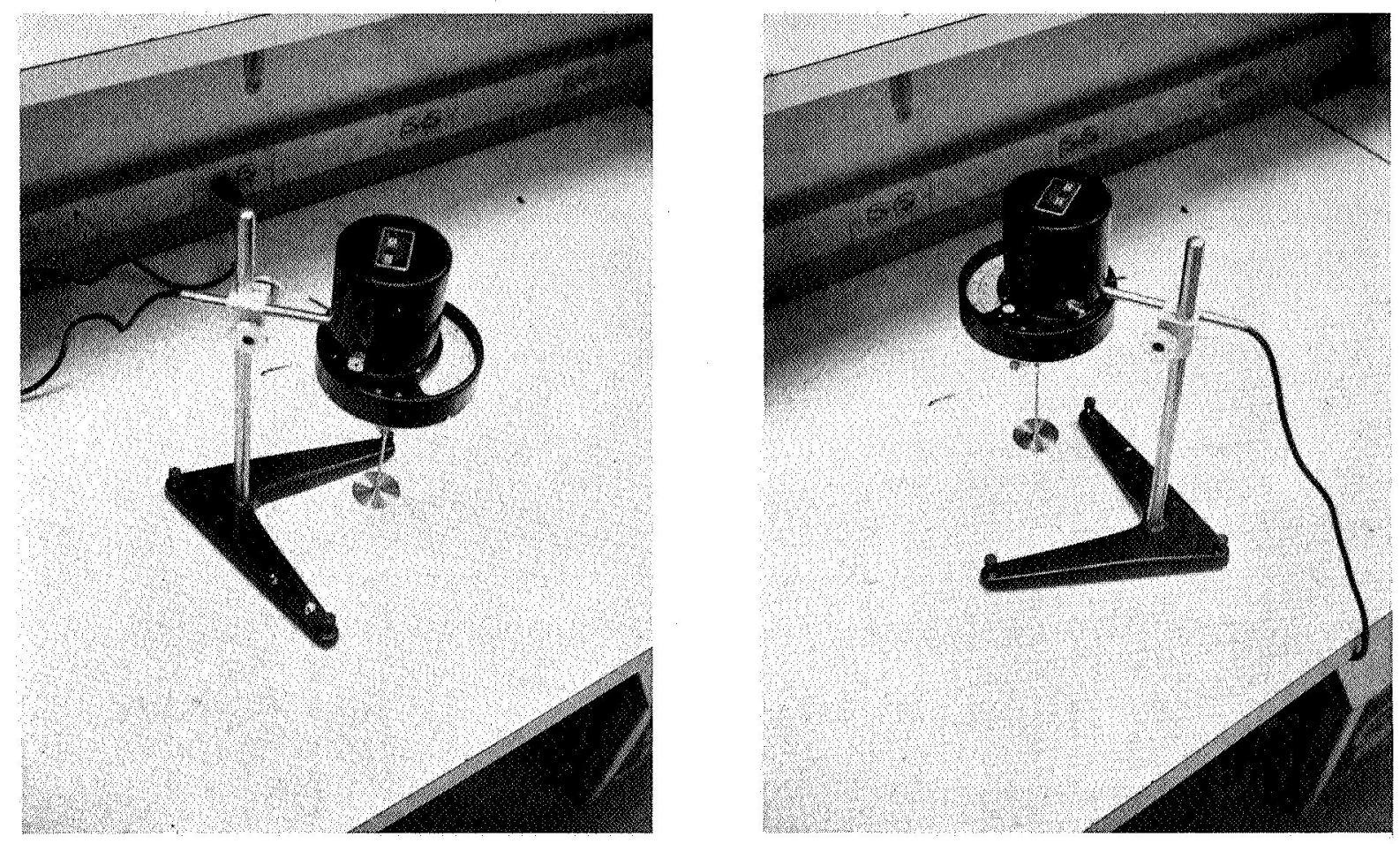

Figure 1. Synchro-Lectric Viscometer 


\author{
Method 115 \\ Photoresist Solids Residue
}

1. Purpose The purpose of this test is to determine the solids content of a given photoresist. It utilizes the evaporation of solvents and differential weighing.

2. Apparatus

2. 1 Analytic Balance with Magnetic Damper

Chainomatic

(Christian Beaker AB-4) Mode1 No. B 1550

Scientific Products Company

(or equivalent)

2. 2 Balance, Triple Beam, Metric Ohaus 760 Order No. B1770 Scientific Products Company

(or equivalent)

2. 3 Bunsen Burner, Heavy Duty

Scientific Products Company

(or equivalent)

2. 4 Desiccator, Scheibler

Model No. 01450-5

(or equivalent)

2. 5 Hot Plate, Electric 6" x. 6",

Tempco, Type 1900

(or equivalent)

2. 6 Oven, Precision

Delco, N-8012, F. O. 31540 No. 25

(or equivalent)

3. Materials

3. 1 Photoresist

3. 2 Photoresist Thinner 
3. 3 Aluminum Weighing Dishes

3. 4 Sand, 30 Mesh White

3. 5 Applicators, Cotton-Tipped

\section{Procedure}

4. 1 For each sample (two samples shall be run on each lot) to be analyzed obtain an aluminum weighing dish and identify by lettering weighing dish flap with lot number.

4. 2 Pour about $10 \mathrm{grams}$ of white sand into weighing dish and get an accurate weight ( $0.1 \mathrm{gr}$ ) of weighing dish, Q-tip and sand. Record lot number and this weight in proper log sheet.

4. 3 Pour 8-10 grams of photoresist into weighing dish and weigh immediately. Record weight in log sheet.

Thoroughly mix photoresist into sand with Q-tip and place on $300^{\circ} \mathrm{C}$ hot plate for 10 minutes.

4. 4 After hot plate place photoresist weighing dish with Q-tip into $105^{\circ} \mathrm{C}$ oven for 16 hours. Record time weighing dish went into oven and time it came out. Take photoresist dish and place in desiccator for 1 hour. Weigh accurately and record weight in $\log$ sheet. Calculate percentage of total solids in following manner:
a. Wt. of dish, sand and stirrer
$=15.7$ grs.
b. Wt. of photoresist, dish, sand and stirrer
$=23.7$ grs.
c. Wt. of baked photoresist, dish, sand and stirrer
$=17.7 \mathrm{grs}$.

Substract (a) from (b) to obtain weight of sample. Substract (a) from (c) to obtain weight of residue.

$$
\begin{aligned}
& \text { Solids } \% \text { by Wt. }=\frac{\text { Wt. of residue } \times 100}{\text { Wt. of sample }} \\
& \frac{2.0 \times 100}{8.0}=25.0 \%
\end{aligned}
$$


5. Càlibration

Zero balances prior to use.

6. Accuracy

Accuracy obtainable is $\pm 1.0 \%$.

\section{Test Frequency}

This test should be performed on a sample from each photoresist lot. 
Method 116

Photoresist Surface Tension

1. Purpose The purpose of this test is to measure the apparent surface tension of the photoresist. This is accomplished by wetting a platimum wire to the resist surface and measuring the force required to break contact.

2. Apparatus

2. 1 Tensiometer,

Catalog No. 70535.

Central Scientific Products, Santa Clara, California, (or equivalent)

2. 2 Beakers,

100,600 and 1000 milliliter.

(any source)

3. Materials

3. 1 Photoresist

3. 2 Photoresist thinner

3. 3 Trichloroethylene

3. 4 Acetone

3. 5 2-Butanone

3. 6 Filter paper

4. Procedure

4. 1 Attach pre-cleaned platinum ring to the lever arm of the tensiometer. (See Figures 1 and 2).

4. 2 Out of the $600-\mathrm{ml}$ beaker containing the raw photoresist, pour about $40-50 \mathrm{ml}$ of photoresist into pre-cleaned $100-\mathrm{ml}$ beaker. Be sure to cover photoresist in $600-\mathrm{ml}$ beaker with tin foil. Place 100-mil beaker on sample table. 
4. 3 With screw (B) in its uppermost position, raise the entire sample table assembly until the ring is immersed about five $\mathrm{mm}$ in the liquid.

4. 4 Lower the entire assembly until the ring is just below the surface of the liquid and approximately centered with respect to the container. Further lower the liquid by means of screw (B) until the ring is on the surface of the liquid and the index is approximately on zero.

4. 5 Increase the torsion of the wire by rotating knob. (A) and at the same time lower the sample table by means of screw (B) so as to keep the index on zero. The index is to be kept on zero even though the surface of the liquid is distended. Continue this double movement until the film breaks.

4. 6 The scale reading at the breaking point of the film is the apparent surface tension $P$. Record this in the proper log sheet.

\section{Calibration}

5. 1 Calibration of Tensiometer

5. 1. 1 Make sure that the instrument is perfectly level before calibrating; if not, adjust knobs on bottom of stand until level ( $L$ ) indicates instrument is level.

5. 1. 2 Clamp the torsion arm with the adjustable stops (D). Hang the dry platinum ring on the hook $(\mathrm{H})$ and cut and fit a small strip of paper on the ring as a platform. Release the torsion arm. Turn the knob (A) until the index (1) and its mirror image are exactly in line with the reference line in the mirror. Loosen the dial clamp (C) and rotate the dial (S) until the vernier (V) indicates approximately zero. Tighten the dial clamp and rotate the dial by means of the fine adjustment $(F)$, until the reading of the vernier is exactly zero.

5. 1.3 Place on the paper platform some accurately known weight of from 500 to 800 milligrams and turn knob (A) until the index (1) is again opposite the reference line on the mirror. Record the dial reading to 0.10 division. Calculate surface tension by use of following formula: 


$$
\begin{aligned}
& M=0.600 \text { gram } \\
& L=6.01 \mathrm{~cm} \text { (use value given in box) } \\
& G=980.3 \mathrm{~cm} / \mathrm{sec}^{2}
\end{aligned}
$$

therefore:

$$
P=\frac{M G}{2 L}=\frac{.6 \times 980.3}{2 \times 6}=49.01 \text { dynes } / \mathrm{cm}
$$

5. 1. 4 If the recorded dial reading is greater than this calculated value, adjust $(G)$ to lengthen the arm. Repeat this calibration procedure, readjusting the zero position with paper in place after each change of (G) until the dial reading agrees with the calculated value. Each unit on the dial represents a surface tension of exactly one dyne per centimeter.

5. 1. 5 When calibration is completed, remove paper from ring and readjust zero position. To readjust zero position turn knob (A) until the index (1) and its mirror image are exactly in line with the reference line on the mirror; loosen the dial clamp (C), and rotate the dial until the vernier is exactly on zero. The tensiometer is now ready for use.

\section{2 Cleaning of Equipment}

Note: In making surface tension measurements one of the most important points is the cleanliness of the ring and the vessels. For accurate work, all glassware and ring must be chemically cleaned.

5.2. 1 Clean a 100- $\mathrm{ml}$ pyrex beaker by pouring $50 \mathrm{ml}$ of TCE to remove any organic residual. Pour contents down the drain.

5.2.2 Pour $50 \mathrm{ml}$ of acetone into beaker to wash off TCE. Pour contents down the drain.

5.2.3 Immerse beaker into hot $\left(95 \pm 5^{\circ} \mathrm{C}\right)$ cleaning solution of chromic acid. Grasping beaker with a set of beaker clamps, rinse thoroughly with tap water then with D.I. Water. Rinse with acetone and blow dry with nitrogen. Beaker is now ready for use. 
5. 2. 4 Clean the platinum ring by rinsing it in 2-Butanone followed by rinsing in D. I. water. Place the bunsen burner in a ventilated hood where no solvents are present in open containers and light. Heat the wire ring in the oxidizing portion of the gas flame until ring turns white hot. Repeat entire step (No. 4) three times. Flame only that portion of the ring which will be immersed in liquid under test. In order to obtain repeat values, ring must be cleaned between each determination.

6. Accuracy

Accuracy obtainable is approximately $\pm 1 \%$.

7. Test Frequency

This test should be performed on a sample from each photoresist lot. 
Method 116

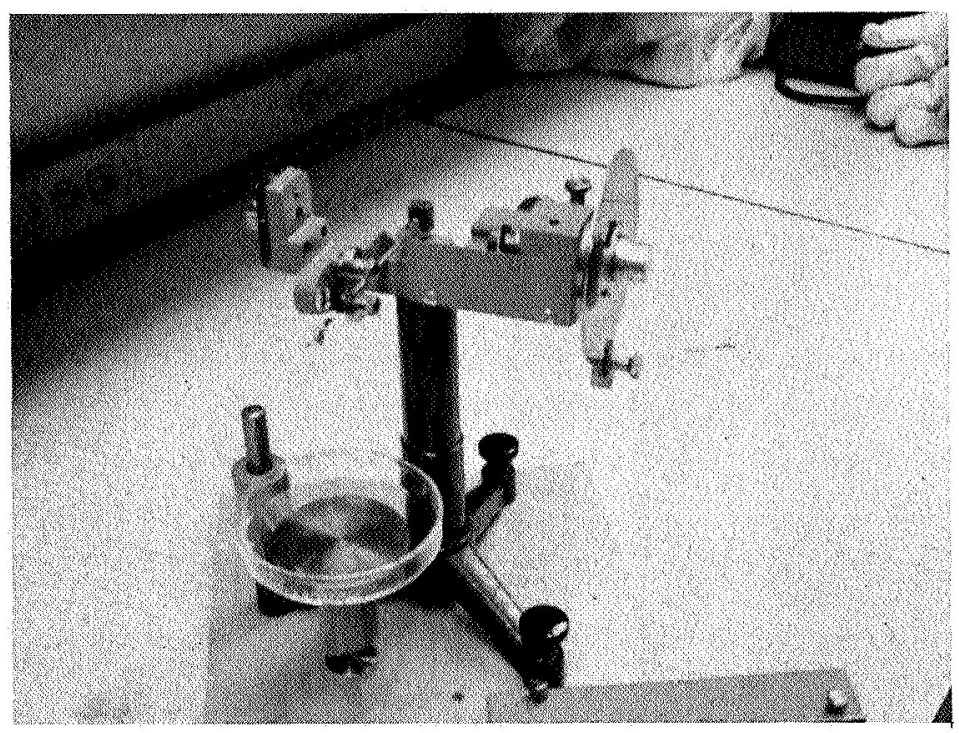

Figure 1. Tensiometer 


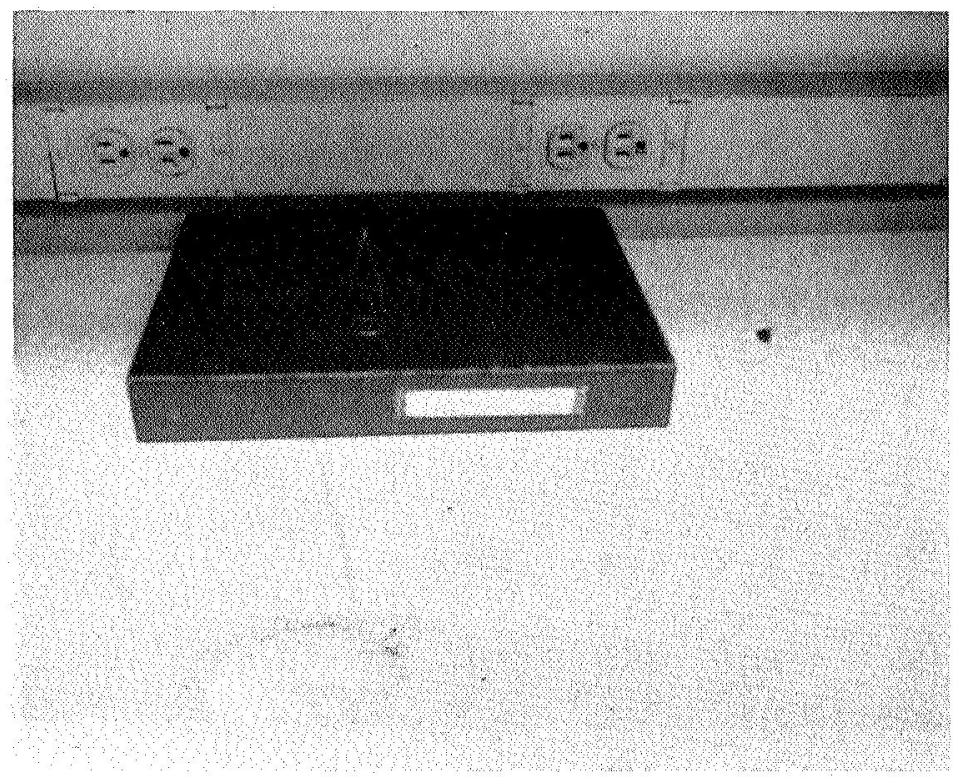

Figure 2. Platinum Wire Ring 


\section{Method 117 \\ Thickness of Epitaxial Layer \\ (Stacking Fault Measurement)}

1. Purpose

The purpose of this procedure is to determine the thickness of the epitaxial layer by means of stacking faults generated at the substrate-layer interface. This method defines the metallurgical interface very accurately for [1 11 ] material. The stacking fault forms an equilateral tetrahedron and the measurement of any side of the triangle when revealed by a light, selective etch is readily translated into the layer thickness:

$$
T=\sqrt{\frac{2}{3}} \quad A
$$

Where $\mathrm{T}=$ altitude of the tetrahedron, in this case, the epitaxial layer thickness

$A=a$ side of the equilateral triangle in the [11] plane. See Figure 1.

2. Apparatus

2. 1 Metallurgical Microscope

Bausch and Lomb, Dynoptic No. 31-20-66-38

(or equivalent)

2. 2 Watson lens (Filar) 2x

Model 1014, London

(or equivalent)

2. 3 Polyethylene beaker (any source) 
3. Materials

3. 1 Hydrofluoric acid, 49\%, Reagent grade.

3. 2 Chromium trioxide $\left(\mathrm{CrO}_{3}\right)$, Reagent grade.

3. 3 Applicators, wood, cotton-tipped

4. Procedure

4. 1 Preparation

4. 1. 1 Prepare a Sirtl etch solution of the following composition: 50 grams chromium trioxide $\left(\mathrm{CrO}_{3}\right)$, $100 \mathrm{ml}$ deionized water, $100 \mathrm{ml}$ hydrofluoric acid. (NOTE: Mixed solution may be stored)

4. 1.2 Twist a cotton-tipped applicator to a fine point.

4. 1. 3 Dip the applicator tip into the Sirtl etch.

4. 1. 4 Touch the applicator to the epitaxial layer surface and deposit a small amount of etchant in the areas to be sampled. (Usually three equally spaced $0.050 \mathrm{mil}$ dots will be adequate for economical production sampling).

4. 1. 5 Allow $15-30$ seconds for the etchant to reach depletion and rinse the wafer under flowing DI water.

4. 1.6 Dry the wafer with a nitrogen gun.

\section{2 Measurement}

4.2. 1 Place the wafer on the microscope stage and locate one of the etched areas in the field of view.

NOTE: "Dark field" illumination should be used for maximum contrast.

4.2.2 Set the counter and the vernier at "zero", to obtain only one image of each stacking fault. 
NOTE: Most faults will be of a uniform size, but smaller ones of random size may be visible. The smaller stacking faults are caused by inclusions or contaminants which initiate an imperfection after epitaxial deposition has started. See Figure 2.

4.2.3 Turn the counter to the nearest whole number where an apex of the second image touches or slightly overlaps an apex of the original image. See Figure 3. If the second image moves in any direction other than parallel to one of the three sides of the triangle, rotate the Watson lens until parallelism is achieved.

4. 2. 4 Adjust the vernier until the two apexes just touch.

4.2. 5 Read the length of the side in whole units and tenths, then compare with calibration to obtain microns.

NOTE: Most Watson Lens, used with a $40 \mathrm{x}$ objective, will show approximately 20 units $=25$ microns

\section{Calibration}

The Watson lens should be calibrated by placing a $B$ and $L$ precision stage micrometer No. 31-16-99 in the apparatus and comparing the number of units per micron.

\section{Accuracy}

6. 1 Required accuracy is \pm 0.5 micron.

6. 2 With correct angular orientation, equipment capability can be \pm 0.2 micron.

7. Test Frequency

This test may be performed on a periodic basis to compare with other methods. 
Method 117

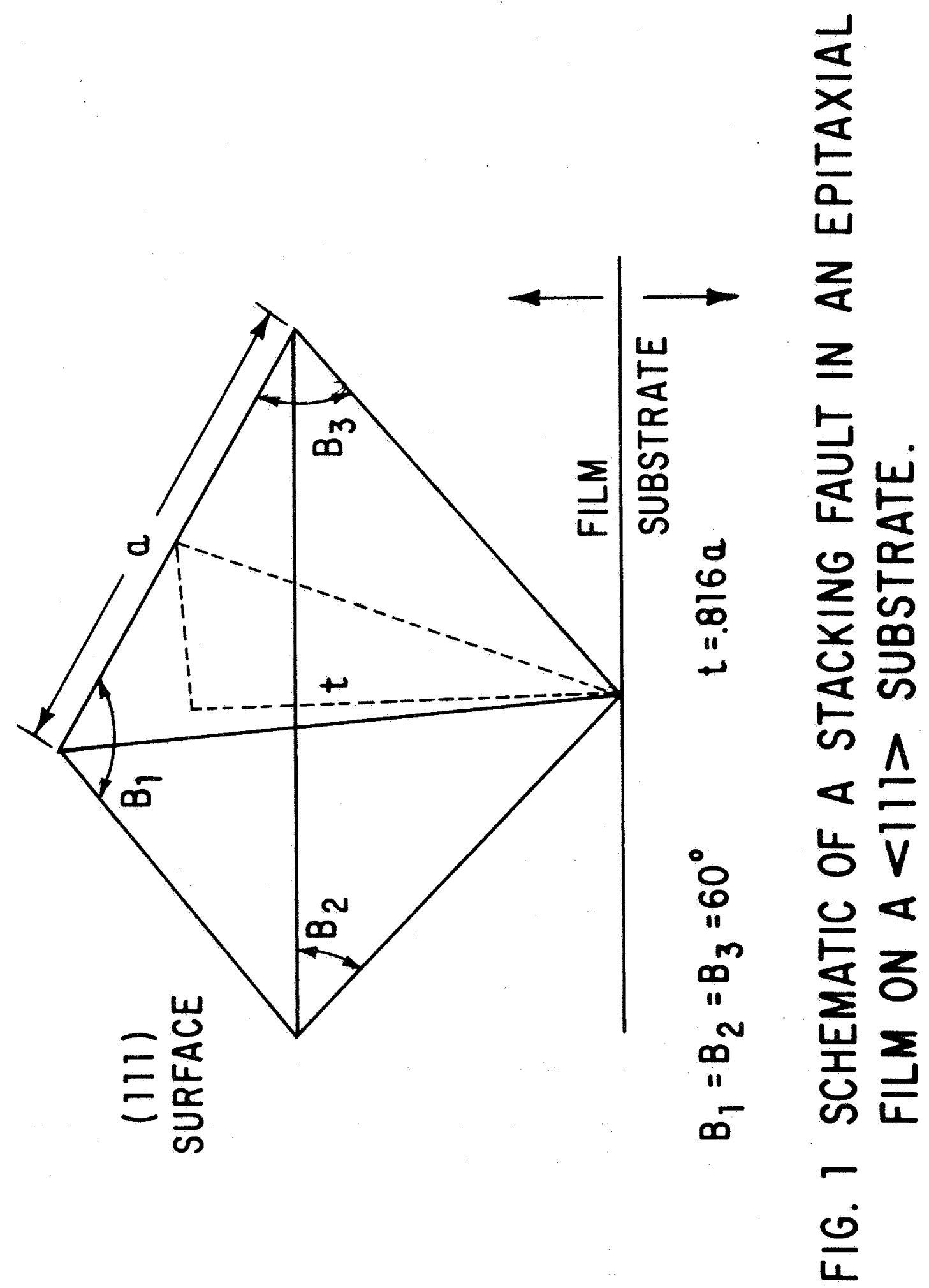



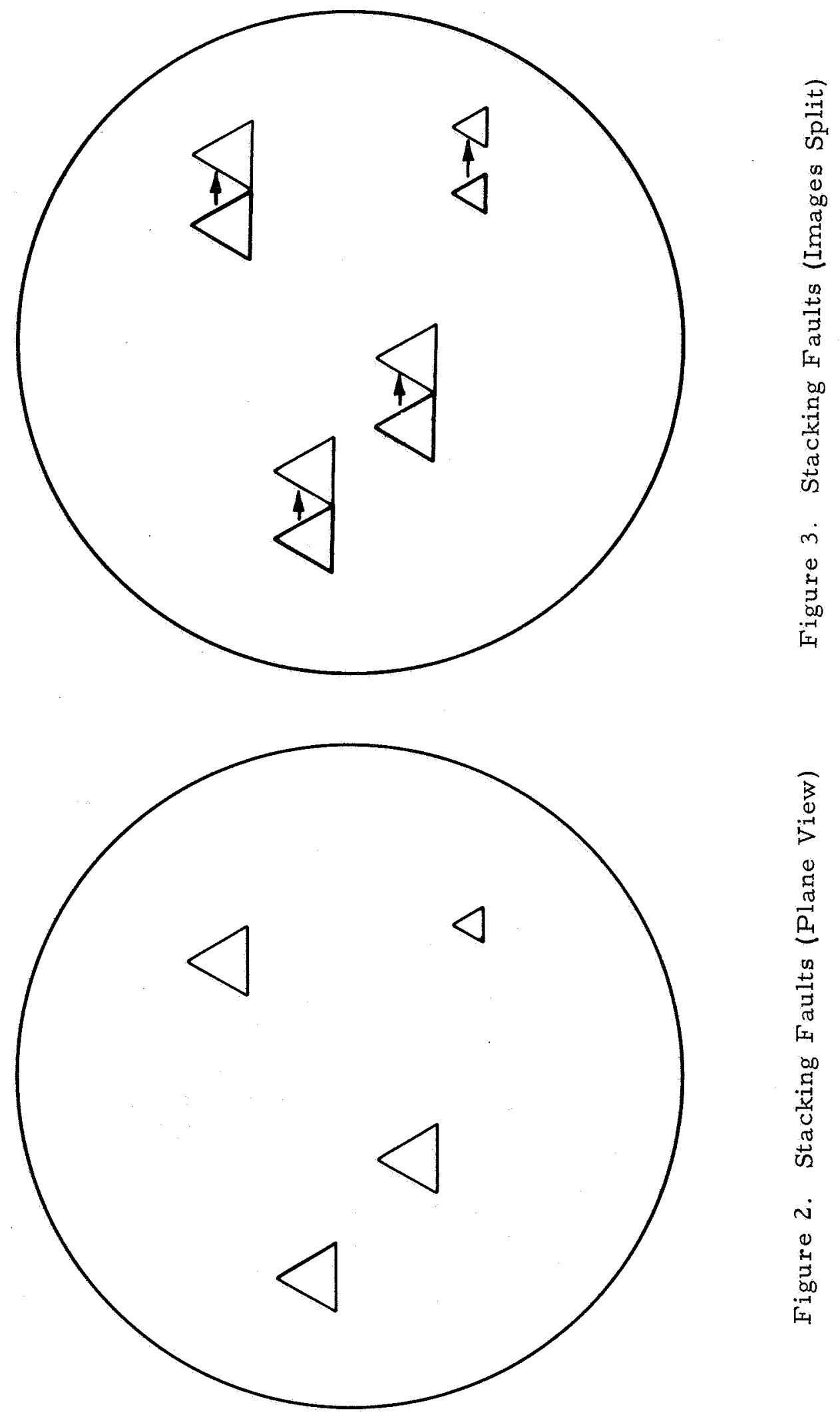

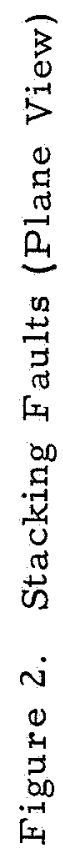


Method 118

Thickness of Epitaxial Layer

(Infrared Spectrophotometer)

1. Purpose

This method may be used to determine the thickness of epitaxial films. It utilizes the reflection of infrared radiation from the wafer substrate.

Output trace from the equipment consists of a series of wavelengths corresponding to intensity maxima and minima. The film thickness is determined by the equation.

$$
\begin{aligned}
& \mathrm{d}=\left(\frac{\mathrm{X}}{6.77}\right)\left(\frac{\lambda \mathrm{a} \lambda \mathrm{b}}{\lambda \mathrm{a}-\lambda \mathrm{b}}\right) \\
& \text { Where } \mathrm{d}= \text { Film Thickness } \\
& \lambda \mathrm{a}, \lambda \mathrm{b}=\text { Any two wavelengths } \\
& \mathrm{X}= \begin{array}{l}
\text { An integer specifying the number of } \\
\text { fringes between the two wavelengths } \\
\text { used in the equation, i. e., b-a }
\end{array}
\end{aligned}
$$

2. Apparatus

2. 1 Infrared Spectrophotometer

Beckman IR - 5A

(or equivalent)

3. Materials

Not applicable. 


\section{Procedure}

\section{1 Infrared Scan}

4. 1. 1 Place a sheet of clean paper in the container under the pen.

4. 1.2 Using tweezers, carefully place the wafer in the holder with the face of the wafer towards the infrared beam. The wafer should be positioned in the slot in such a way that it completely blocks the path of the infrared beam.

4. 1.3 Make sure that the re is sufficient ink in the well attached to the pen.

4. 1. 4 Check that the pen is in the raised position. The pen may be raised from the paper by using the pen lift lever, which is situated at the lower end of the pen carriage.

4. 1. 5 Move the pen carriage to the left end of its traverse.

4. 1.6 Turn the function switch to PEN.

4. 1.7 Adjust the slope of the shutter in the reference space until the pen moves to a position of approximately 75 on the vertical (ordinate) scale of the graph paper.

4. 1.8 Move the pen lift lever to lower the pen on to the graph paper.

4. 1.9 Turn the function switch to the START position, from which it will return automatically to SCAN by spring action. The scan will then occur throughout the instrument range from $11 \mu$ to $35 \mu$.

4. 1. 10 Allow the scan to occur until the pen carriage reaches the end at $35 \mu$ when it will stop automatically. The automatic scan stop also lifts the pen from the chartpaper. 
4. 1. 11 Remove the wafer from the holder.

4.1.12 Place another wafer in the holder and repeat steps 4.1 .2 to 4.1 .11 . However, in step 4.1.7, position the pen at approximately 50 on the ordinate axis for the second wafer and at about 25 for the third wafer.

4. 1. 13 Replace the paper on which the trace is made after three wafers.

4. 1. 14 Do not touch any of the dials on the instrument except the function switch. The other dials will be set by the Beckman Service representative on his monthly visits.

4. 1. 15 Turn the function switch to the OFF position upon completion.

\section{2 Thickness Calculation}

4.2. 1 Find the first clear peak which is traced at the left of the chart. Determine its wavelength from the scale at the bottom of the chart.

4.2. 2 Find the last clear peak on the right and obtain its wavelength.

4.2.3 Count the number of fringes (cycles) between the peaks chosen.

4. 2. 4 Using the formula from paragraph one, calculate the epitaxial layer. See Figure 1 for example.

4.2. 5 For very thin epitaxial layers (about six microns or less) only one peak and one valley may be obtained. If this is the case, use the chart as if the reading was across one whole fringe. Then divide the thickness calculated by two in order to give the correct epitaxial thickness value.

\section{Calibration}

See individual equipment manufacturer's instructions. 
6. Accuracy

6. 1 Required accuracy is \pm 0.5 microns.

6.2 Satisfactory accuracy is obtainable if substrate resistivity is less than $0.025 \Omega-\mathrm{cm}$ and layer is greater than 5 microns.

7. Test Frequency

This test, or an equivalent, should be performed on every epitaxial film. 


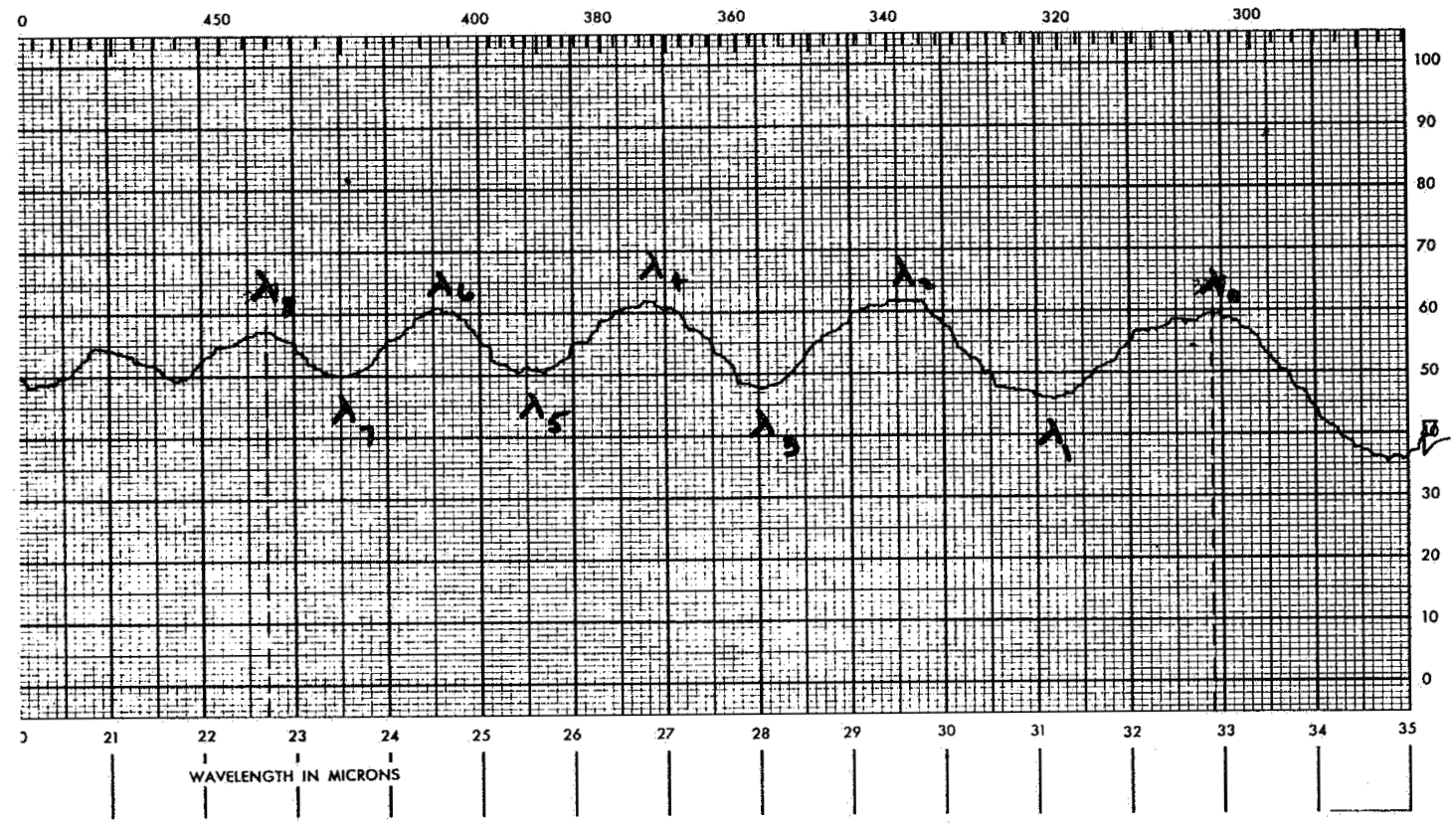

Figure 1. Infrared Spectrophotometer Trace

$$
\begin{aligned}
\lambda_{0} & =32.9 \mu \\
\lambda_{8} & =22.7 \mu \\
\mathrm{x} & =4 \\
\mathrm{~d} & =\left(\frac{\mathrm{x}}{6.77}\right)\left(\frac{\lambda_{0} \lambda_{8}}{\lambda_{0}-\lambda_{8}}\right) \\
& =\left(\frac{4}{6.77}\right)\left(\frac{32.9 \times 22.7}{32.9-22.7}\right) \\
& =43.7 \mu
\end{aligned}
$$




\section{Method 119 \\ Thickness of Epitaxial Layer \\ (Nomarsky Polarization Interferometry)}

1. Purpose This test may be used to measure extremely thin ( 0.6 to 2.6 microns) epitaxial films. It utilizes interference fringes from two images of the junction formed by monochromatic light passing through a double quartz prism. The layer thickness may be obtained from:

$$
d=\left(n+\frac{\Delta S}{S}\right) \frac{\lambda}{2}
$$

$$
\begin{aligned}
& \text { where } \mathrm{d}=\text { Layer thickness } \\
& \mathbf{n}=\quad \text { Number of fringes } \\
& \Delta S=\text { Fringe displacement } \\
& S=\quad \text { Fringe spacing } \\
& \lambda=\text { Wavelength of incident light }
\end{aligned}
$$

2. Apparatus

2. 1 Metallurgical Microscope

Reichert No. 304-758

(or equivalent)

2. 2 Nomarsky polarization interferometer

Reichert No. 34-973

(or equivalent)

2. 3 Filter, orange sodium, $5890 \AA$

Reichert

(or equivalent) 
2. 4 Ball-grooving equipment Figure 1.

(or equivalent)

2. 5 Microscope Camera

Polaroid Land

(or equivalent)

\section{Materials}

3. 1 Hydroflouric acid, concentrated, Reagent grade.

3. 2 Nitric acid, concentrated, Reagent grade.

3. 3 Cupric Chloride, Reagent grade $\left(\mathrm{CuCl}_{2} \cdot 2 \mathrm{H}_{2} \mathrm{O}\right)$.

3. 4 Trichloroethylene, Electronic grade.

3. 5 Polishing compound, 1 micron, Linde "A".

3. 6 Polishing compound, 0.3 micron, Linde " $\mathrm{B}$ ".

\section{7 Thinner, Dymo.}

4. Procedure

\section{1 Preparation}

4. 1. 1 Tape test wafer (N/P or $P / N$ to glass slide and identify).

4. 1.2 Mix a small quantity of grooving solution in the following proportions:

$$
\begin{array}{ccc}
12 \text { parts } & 0.3 \text { micron polishing compound } \\
1 \text { part } & 1 & \text { micron polishing compound } \\
20 \text { parts } & - & \text { Dymo thinner }
\end{array}
$$

4. 1.3 Place a small drop of a grit mixture on wafer near its center.

4. 1. 4 Turn the ballmotor on. 
4. 1. 5 Clean the ball with TCE using a cotton bud.

4. 1.6 Center ball over grit drop. Lower ball gently on to the wafer while holding slide steady. (A $1 \mu$ layer requires 3 - 4 seconds of grooving without additional pressure to the ball).

4. 1. 7 Using TCE and cotton buds, clean off grit from wafer surface. Using clean buds dipped in TCE, clean out groove.

4. 1.8 Mix a small quantity of staining solution in the following proportions:

$$
\begin{array}{ll}
55 \mathrm{ml} & \mathrm{HF} \\
13 \mathrm{mg} & \mathrm{CuCl}_{2} \cdot 2 \mathrm{H}_{2} \mathrm{O} \\
1 \mathrm{drop} & \mathrm{HNO}_{3}
\end{array}
$$

4. 1. 9 Place one drop of stain solution in groove.

4. 1. 10 Place wafer under collimated light. When groove turns black, dip slide into beaker of water. Dry slide and wafer carefully with paper towel.

4. 1. 11 Groove is ready to be examined under microscope $(20 x$ to $50 x)$. If groove or staining are not satisfactory, repeat 4.1 .3 to 4.1 .10 .

\section{2 Measurement}

4.2. 1 Place the wafer on microscope stage (Reichert). Make sure that Nomarski interferometer and orange sodium filter are in place as well as $2 \mathrm{np}$ lens.

4.2. 2 Switch the transformer on and set the current to maximum.

4.2. 3 Focus the binocular on wafer edge and then slide the wafer until the groove is visible.

4. 2. 4 Select the best polished part of the groove and turn it till the dark interference fringes outside the groove are almost perpendicular to the groove edge. 
4.2. 5 Turn the binocular off and observe the groove in the horizontal eye piece near the camera.

4.2.6 Adjust the position of the wafer to focus the interference pattern in order to get the best definition of the junction. The junction is the dark area between the fringes in the fringe field.

4. 2. 7 Make a photomicrograph of the epitaxial layer.

4. 2. 8 On a picture showing interference fringes, count the number of whole fringes between the groove edge and the junction. This gives the thickness fringe count to the nearest whole number of fringes.

4.2.9 In order to determine the final fringe fraction, extend two adjacent straight edges into the groove and measure the distance " $S$ " between them. Figure 2 .

4.2. 10 The junction line described above has regions of light and dark coloring. In the dark regions, the junction line narrows and in the light regions, it widens. Along the junction, measure the distance between the reference line drawn above and the start and the end of a nearest light section of the junction ( $x$ and $y$ ).

4.2.11 Distance measurement would have a + sign if it has the same direction as fringe entering the groove or a - sign if it is to the opposite side of reference line.

4.2.12 Respecting their signs the ratio $\frac{ \pm x \pm y}{2 S}$ gives the fraction of the fringe to add or substract from the whole number of fringes.

4. 2.13 Thickness is determined from Table 1: (sodium light $) \quad(\lambda=5890 \AA)$. See Figure 3 for example. 


\section{Calibration}

Not applicable.

6. Accuracy

6.1 Required accuracy is 0.1 micron.

6.2 Accuracy obtainable is 0.05 micron.

\section{Test Frequency}

This test should be performed on a sample of every reactor run of thin epitaxial layers. 


\begin{tabular}{|c|c|}
\hline 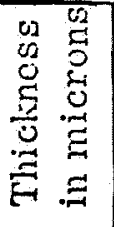 & 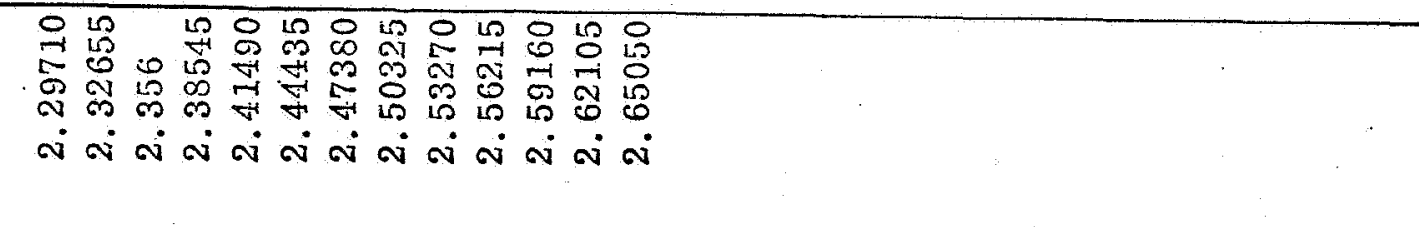 \\
\hline 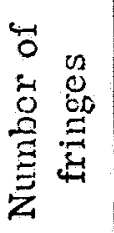 & 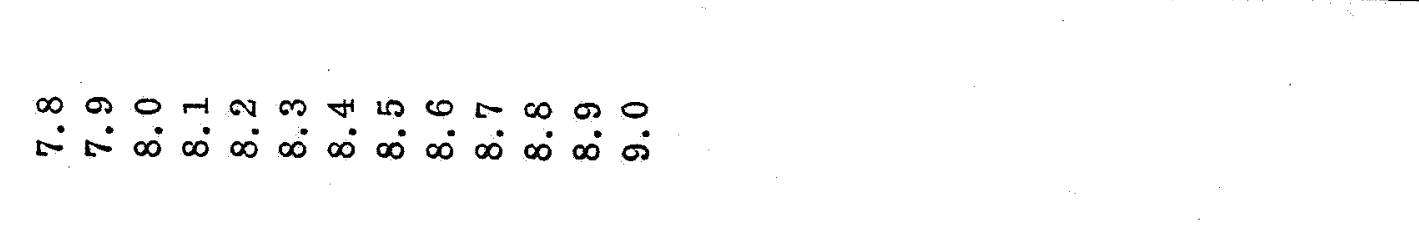 \\
\hline 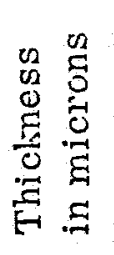 & 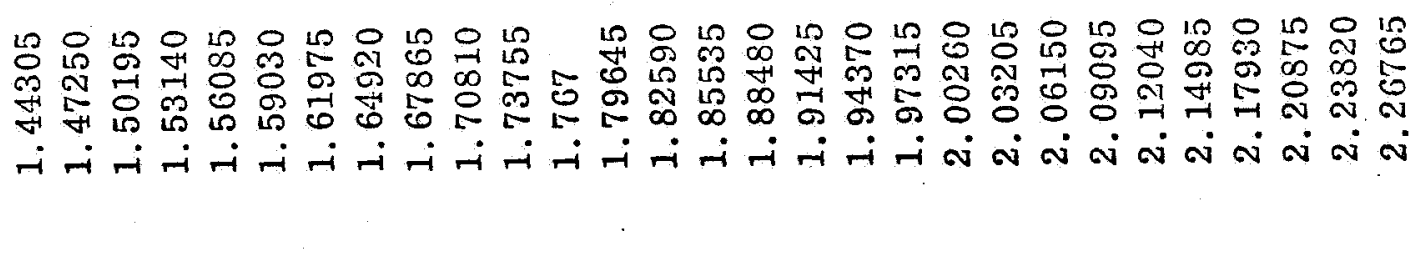 \\
\hline 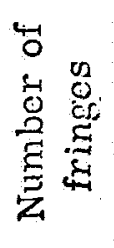 & 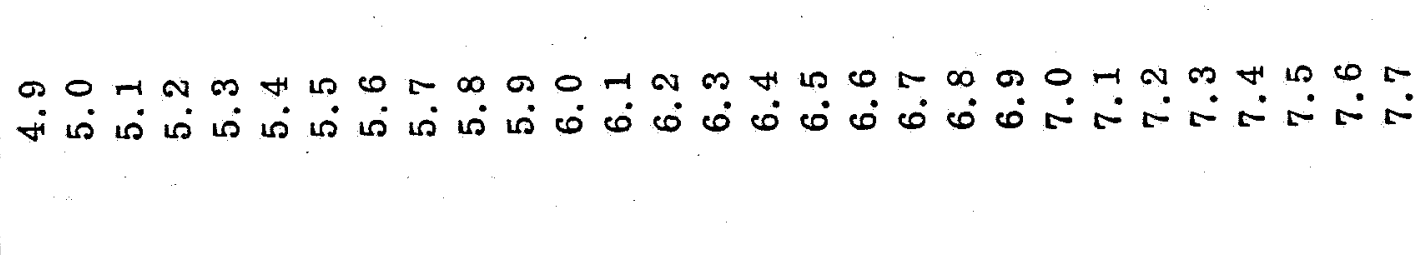 \\
\hline 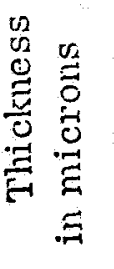 & 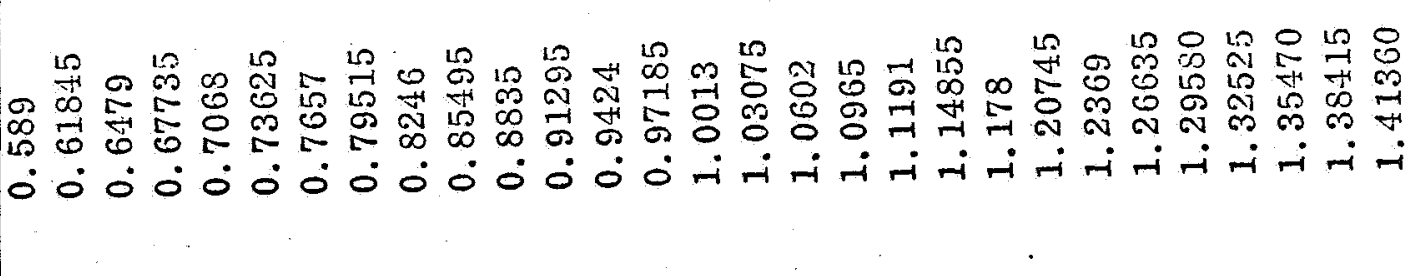 \\
\hline 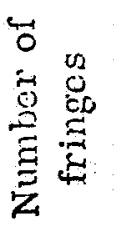 & 率 \\
\hline
\end{tabular}




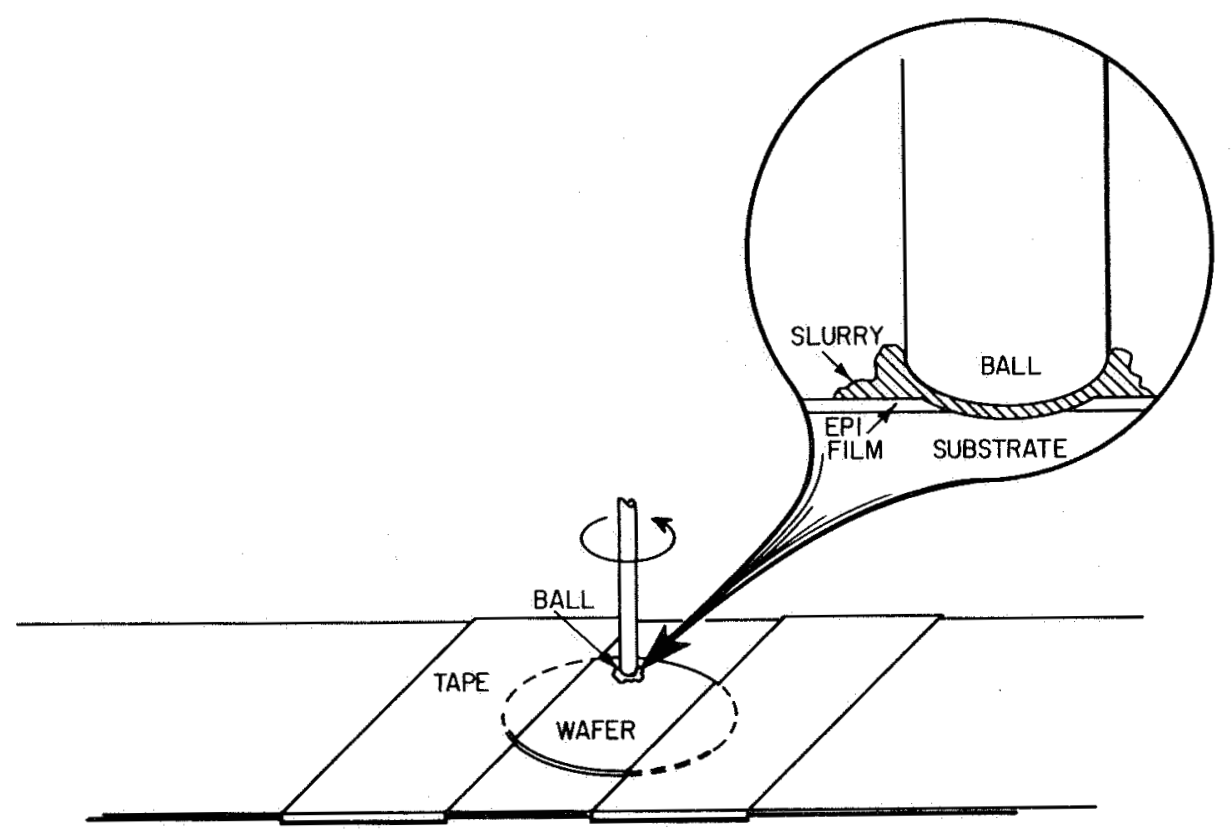

Figure 1. Grooving Setup 


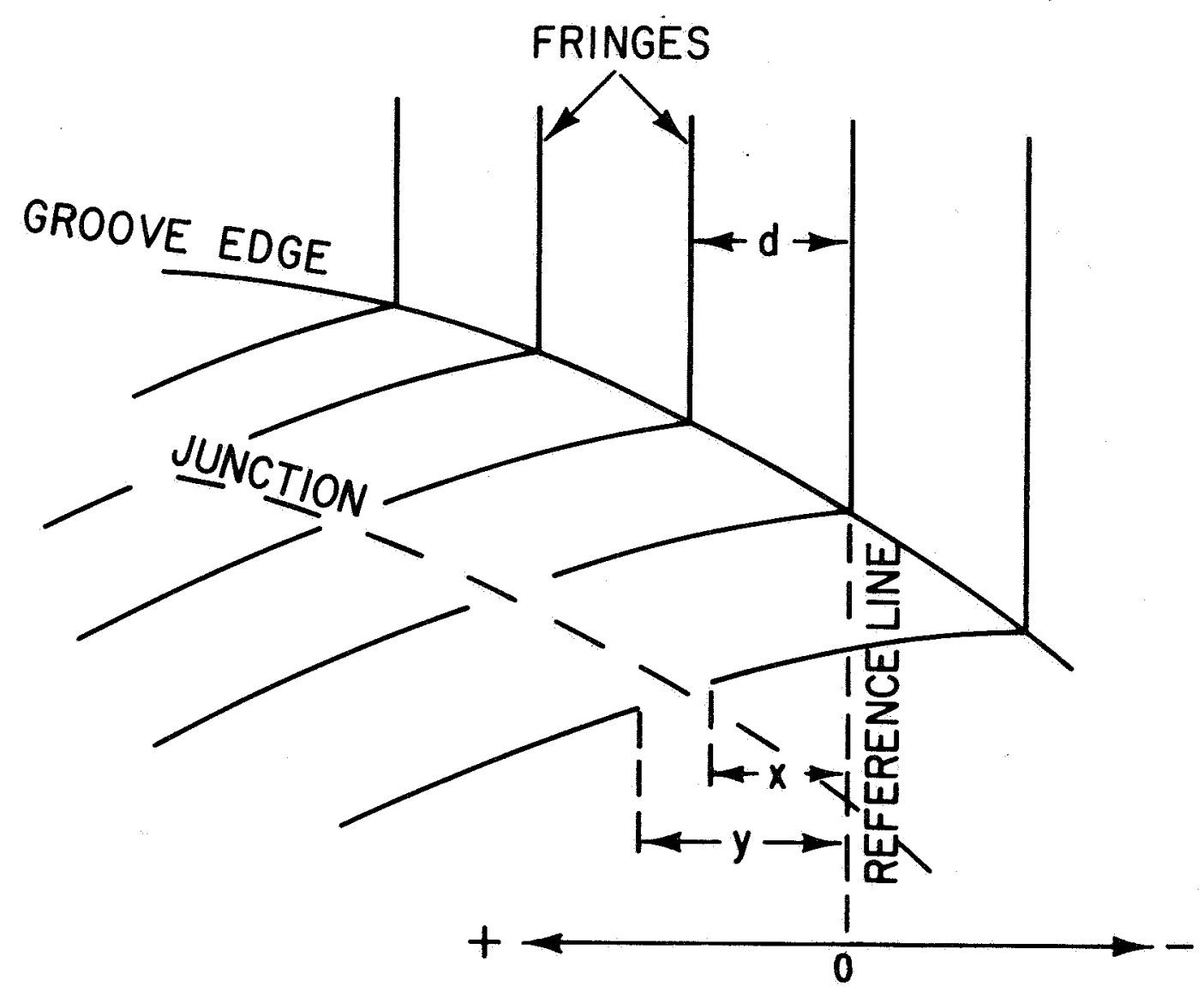

Figure 2. Nomarsky Fringes Graphic Representation 


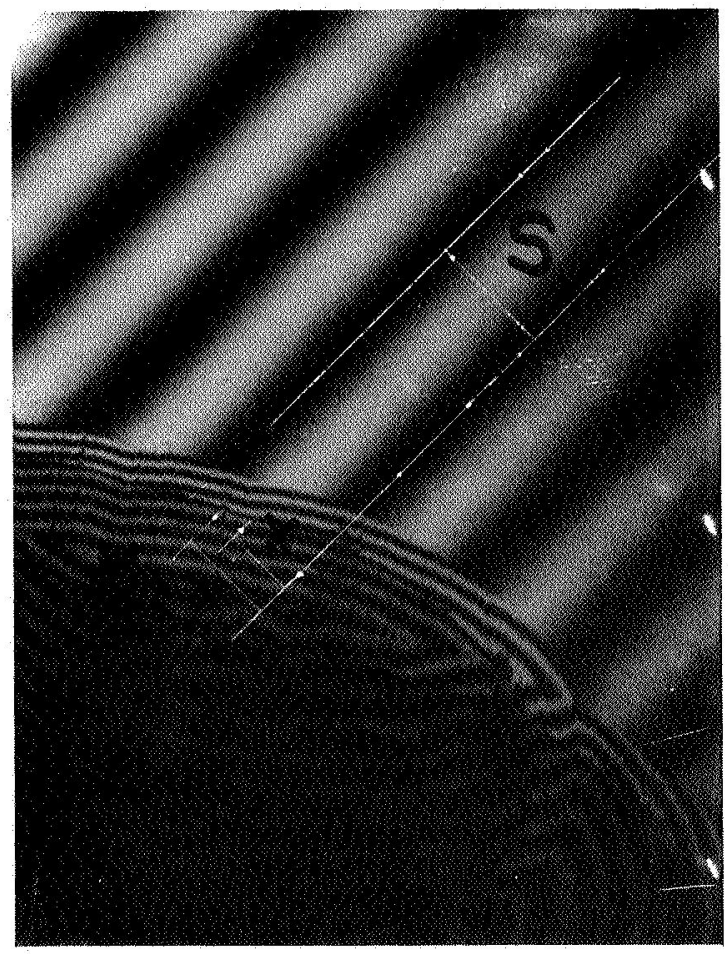

Figure 3. Nomarsky Fringes (Actual)

$$
\begin{aligned}
& \lambda=5890 \AA \text {, Sodium } \\
& S=0.50 \text { inches } \\
& x=0.28 \text { inches } \\
& y=0.40 \text { inches } \\
& n=2 \text { Fringes }
\end{aligned}
$$

$$
\begin{array}{rlrl}
\Delta S & =\frac{x+y}{2} & \frac{+x+y}{2 S} & =\frac{0.28+0.40}{2 \times 0.50} \\
& =\frac{0.28+0.40}{2} & & =0.68 \\
& =0.34 & & 0.70 \\
d & =\left(2+\frac{0.34}{0.50}\right)\left(\frac{5890}{2}\right) & \text { From Table I: } \\
& =(2.68)(2945) & d & =0.795 \text { micron } \\
& =7890 \AA & & \\
& =0.789 \text { micron } & &
\end{array}
$$


Method 120

\section{Resistivity of Epitaxial Layer}

(Four-Point Probe)

1. Purpose

This method may be used to determine the resistivity of any epitaxial film. If the film is of opposite conductivity from the substrate, the measurement is direct; if not, the resistivity is indirectly measured through use of a substrate of opposite type. A four-point probe is used to obtain sheet resistance for a film of known thickness, which yields resistivity:

$$
\begin{aligned}
& \rho=A \frac{V}{I} \quad A=F(W, S) \\
& \text { If } W<<S \\
& \rho=4.53 \mathrm{~W} \frac{V}{I}
\end{aligned}
$$

Where: $\quad W=$ Film thickness

$$
\begin{aligned}
\mathrm{S} & =\text { Probe spacing } \\
\frac{\mathrm{V}}{\mathrm{I}} & =\text { Sheet resistance of the layer }
\end{aligned}
$$

\section{Apparatus}

2. 1 Four-point probe, 0.040 inch spacing

Dumas

(or equivalent)

2. 2 Power supply, constant current 0.1, 1. 0, 10 mA ranges (any source) 


\section{3 Voltmeter, Digital \\ Fairchild 7100 \\ (or equivalent)}

\section{Materials}

3. 1 Glass microscope slides, $1^{\prime \prime} \times 3^{\prime \prime}$

\section{Procedure}

4. 1 Process a check wafer through the epitaxial reactor to be evaluated.

4. 2 Place the wafer to be measured on the glass slide under the four-point probe head with the epitaxial layer side up. Center the wafer so that no probe point is closer than one-eighth inch from the edge of the wafer. See Figure 1.

4. 3 Set the constant current selector to $0.1 \mathrm{~mA}$.

4. 4 Turn the constant current generator switch on.

4. 5 Screw the four-point probe down on the wafer using the end ratchet until the proper contact causes the ratchet to slip.

4. 6 Check to see if the constant current meter is indicating full scale. If not, raise the probe and repeat step 4.5

4. 7 Switch the polarity of the current generator from plus to minus and observe the current reading for any variation.

4. 8 If full scale current readings are obtained in both positions (i. e., plus and minus), read the indicated $V / I$ on the voltmeter.

4. 9 The V/I readings should be within $10 \%$ of each othe $r$ when the polarity of the current generator is reversed. If this is not so, release the pressure on the probe head one-fourth turn of the ratchet at a time until the readings do agree within $10 \%$.

4. 10 Take three V/I readings on each check wafer, average and record. See Figure 2 for example. 


\section{Calibration}

5. 1 Probe Spacing

5. 1. 1 Cover a $1^{\prime \prime} \times 3^{\prime \prime}$ glass microscope slide with aluminum foil.

5. 1.2 Obtain an impression of the four-point probe points.

5. 1.3 Measure spacing with any optical comparator.

5. 1. 4 Adjust spacing per probe manufacturer's instruction.

\section{Accuracy}

Obtainable accuracy is $\pm 10 \%$ with groove-and-stain thickness determination.

\section{Test Frequency}

This test should be performed on at least one check wafer from every epitaxial reactor run. 


\section{FOUR POINT PROBE - SET-UP}

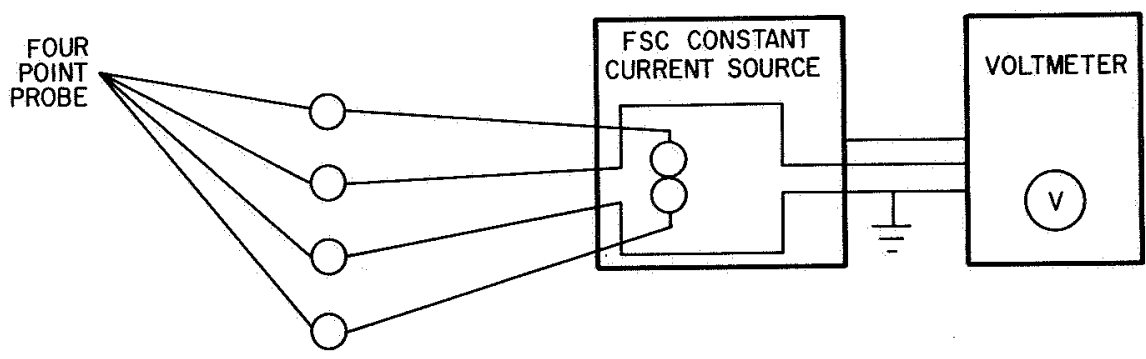

Figure 1. 


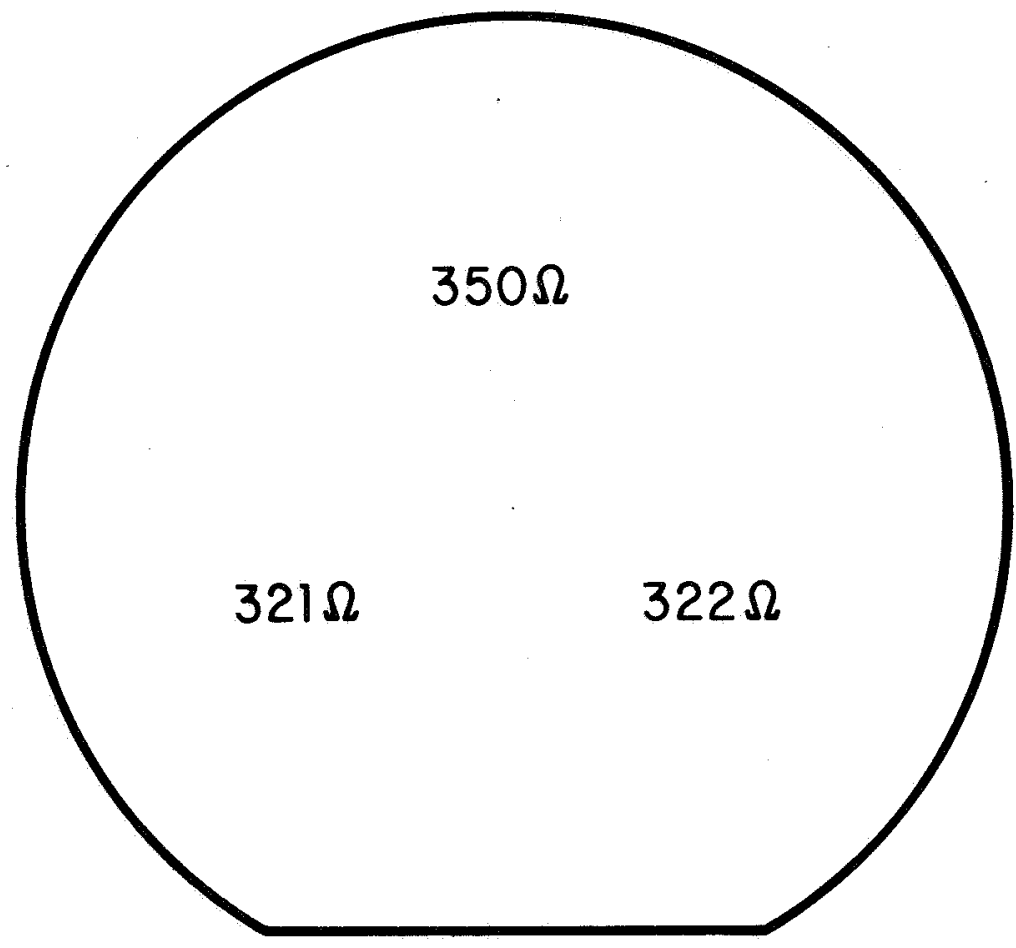

Figure 2. Sample Resistivity Calculation

$$
\begin{aligned}
\frac{V}{I} & =\frac{350+321+322}{3}=331 \\
W & =10 \text { microns }=10^{-3} \mathrm{~cm} \\
\rho & =4.53 \mathrm{~W} \frac{\mathrm{V}}{\mathrm{I}} \\
& =4.53 \times 10^{-3} \times 331 \\
& =4.53 \times 10^{-3} \times 3.31 \times 10^{2} \\
& =1.5 \Omega \mathrm{cm}
\end{aligned}
$$


Method 121

Epitaxial Layer Surface Defects

1. Purpose This method may be used to evaluate surface condition of the epitaxial layer.

2. Apparatus

2. 1 Metallurgical Microscope

Bausch and Lomg, Model 31-20-68-38 (DMETR)

(or equivalent)

3. Materials

3. 1 Chromium Trioxide, Reagent grade.

3. 2 Hydrofluoric Acid, Electronic grade.

3. 3 Deionized Water.

4. Procedure

4. 1 Place wafer to be examined on microscope stage.

4. 2 Using a minimum of $50 x$, scan surface of sample.

4. 3 Note the number found of the following defects:

4.3. 1 Stacking Faults - Thin, straight lines in a plane forming one or more sides of equilateral triangles, with parallel orientation.

4.3.2 Pyramids - Any pointed, convex defect of a pyramidal or tripyramidal shape. 
4.3.3 Pits - Any concave defect from etching or other cause.

4.3.4 Granular Surface - Epitaxial layer deposited rough or unpolished surface.

4.3. 5 Scratches - Long scratches or gouges in the surface.

5. Calibration

None.

6. Accuracy

Not applicable.

7. Test Frequency

All wafers should be inspected for these defects. 
Method 122

Epitaxial Process

(Substrate Temperature)

1. Purpose This method may be used to determine the substrate temperature during epitaxial deposition. It utilizes the comparison of infrared radiation (incandescence) of a filament of known resistivity and the object to be measured.

2. Apparatus

2. I Optical Pyrometer

Pyro Micro-Optical Pyrometer

The Pyrometer Instrument Co., Inc.

(or equivalent)

3. Materials

Not applicable.

4. Procedure

4. 1 Set the telescope a convenient distance from the epitaxial reactor to be measured. (less than 5 feet).

4. 2 Connect lead wires in series to terminals of telescope, meter and battery, in that order. See Figure 1.

4. 3 Focus the telescope:

4.3. 1 Set scale range knob and red filter knob at position No. 1.

4.3.2 Turn rheostat up enough to cause the pyrometer lamp filament to become weakly luminous when looking through the ocular.

4. 3. 3 Turn the ocular ring to bring the apex of the filament into focus. 
4.3. 4 Focus on the substrate to be measured by adjusting the distance knob.

4.3. 5 Align the filament and substrate image.

4. 4 Set red filter knob to position No. 2 .

4. 5 Set range scale to $1200-1500^{\circ} \mathrm{C}$.

4. 6 Adjust the rheostat until the filament blends with the substrate.

4. 7 Read the substrate temperature directly from the meter.

4. 8 Disconnect battery when measurement is complete.

\section{Calibration}

5. 1 Galvanometer Zero Calibration

5. 1. 1 Connect a precision milliameter in series with the circuit.

5. 1.2 Adjust the rheostat until the meter pointer corresponds to the milliampere sensitivity points indicated on the scale.

5. 1.3 Remove cover plate and turn zero adjuster until points coincide with meter readings.

\section{Accuracy}

Accuracy obtained will be $\pm 2^{\circ} \mathrm{C}$.

\section{Test Frequency}

This test or an equivalent should be performed during each epitaxial deposition. 
Method 122

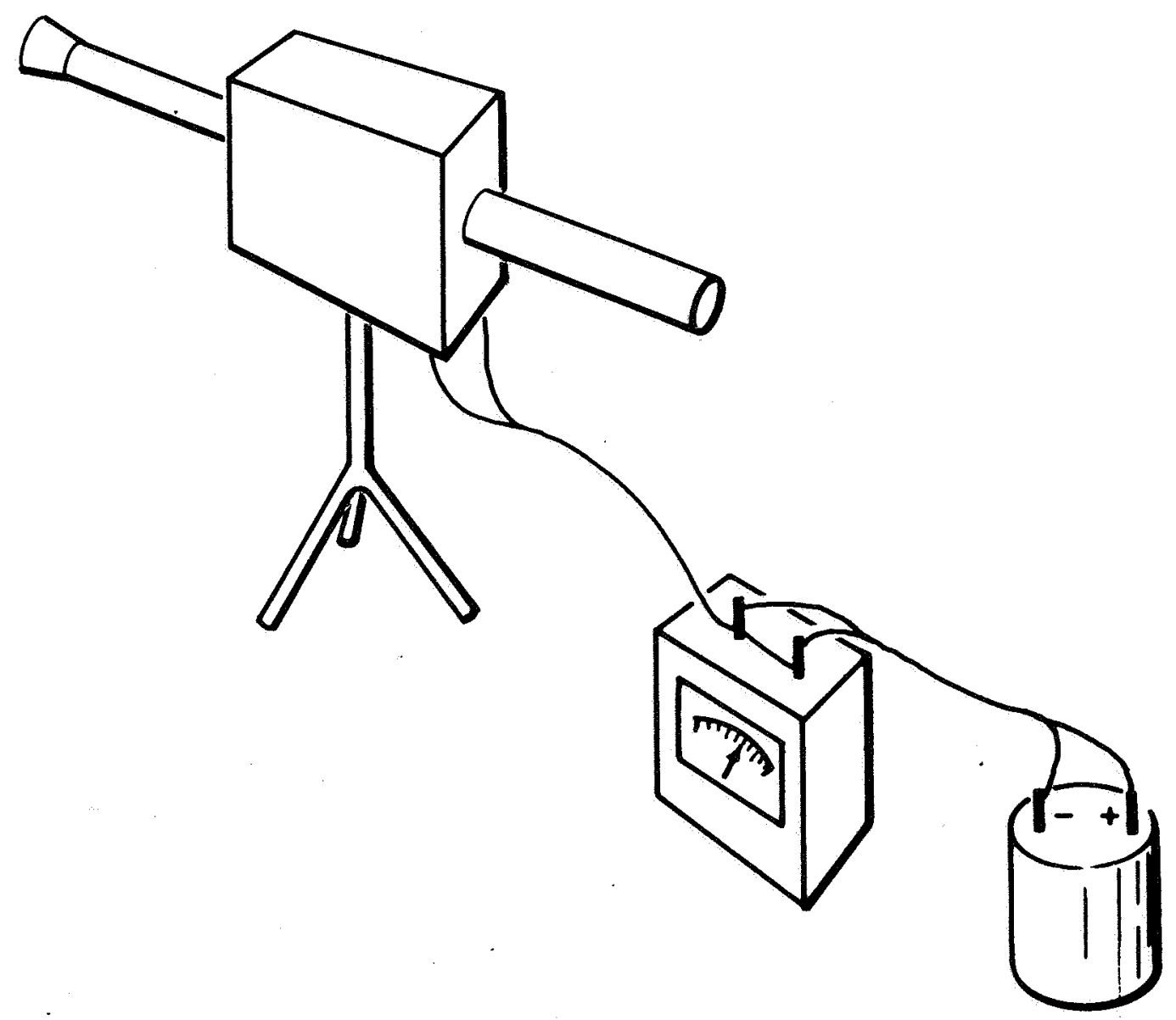

Figure 1. Optical Pyrometer 
Method 123

Thickness of Passivating Layer

(Tolansky Interferometer)

1. Purpose

This test procedure is applicable to the measurement of any thin film, whether transparent or not. It utilizes the interference of monochromatic light introduced into the optical system through a 95 percent mirror and reflected between that and the metallized specimen. The two mirrors are inclined to one anothe $r$ causing the interference fringes to be normal to the edge of the passivating layer. The horizontal displacement of any fringe enables one to calculate the layer thickness by the following relationship:

$$
\frac{\mathrm{x}_{0}}{\mathrm{D}}=\frac{\frac{\lambda}{2}}{w}
$$

Where $x_{0}=$ Layer thickness

$\lambda=$ Wavelength of incident light

$\mathrm{w}=$ Distance between fringes

$D=$ Distance any fringe is displaced See Figures 1 and 2 .

2. Apparatus

2. 1 Metallurgical microscope, metalstar trinocular body AO No. $2200 \mathrm{~T}-4$. American Optical Company (or equivalent) 
2. 2 Polaroid Camera AO No. 1052A.

American Optical Company

(or equivalent)

2. 3 Interferometer Jig

Fairchild design

See Figure 3.

2. 4 Monochromatic light source

2.4.1 Mercury, $5461 \AA$

LaPine Scientific Company, Model 216-01

(or equivalent)

2. 4.2 Sodium, $5893 \AA$ (optional)

LaPine Scientific Company, Mode1 215-96

(or equivalent)

2. 5 Hot plate, 6" $\times 6$ 6", electric

Temco type 1900

Corning, Pyroceram PC-35

(or equivalent)

2. 6 Polethylene beaker

(any source)

3. Materials

3. 1 Hydrofluoric acid, 49 percent Reagent grade.

3. 2 Acetic acid, glacial, Reagent grade.

3. 3 Trichloroethylene, Electronic grade.

3. 4 Acetone, Electronic grade.

3. 5 Apiezon wax.

3. 6 Slides, glass microscope, $1 " \times 3 "$. 


\section{Procedure}

\section{1 Preparation}

4. 1. 1 Process a polished test chip through the passivation (oxidation, etc.) operation (s) to be evaluated.

4. 1. 2 Place a glass slide on a hot plate adjusted to a temperature slightly above the melting point of the apiezon wax $\left(150-160^{\circ} \mathrm{C}\right)$.

4. 1. 3 Place the test chip on the heated slide with the polished side up.

4. 1. 4 Mask about one-fourth of the chip area with black wax.

4. 1. 5 Carefully remove the test chip from the glass slide.

4. 1. 6 Cool the chip under running water for 15 seconds.

4. 1. 7 Immerse the chip in a prepared solution of three parts acetic acid to two parts hydroflouric acid until all unmasked oxide is removed (four to eight minutes).

4. 1.8 Rinse chip in running water for five to ten seconds.

4. 1. 9 Remove wax from chip with trichloroethylene.

4. 1. 10 Rinse test chip in acetone and allow to dry.

4. 1. 11 Evaporate a $1000 \AA$ silver or aluminum film over the film to be examined.

NOTE: While silver has a higher index of reflectivity, it must not be allowed to tarnish prior to measurement.

\section{2 Measurement}

4.2. 1 Place the specimen on the platform, mirrored side up.

4.2.2 Rotate the specimen on the platform until the step to be measured is normal to the front edge of the fizeau plate. 
4.2.3 Raise the platform until the specimen is in contact with fizeau plate by turning the knurled brass elevation nut. This will give maximum fringe spacing.

4.2. 4 If a smaller fringe spacing is desired, the platform may be raised slightly, reducing the wedge angle, $\Theta$, and as a result, fringe spacing.

4. 2. 5 Measure distance between fringes on the photomicrograph with a precision rule. (w)

4.2.6 Measure distance which any fringe is displaced as it passes across the film step.

4. 2. 7 Calculate film thickness, $X_{0}$, using the relationship:

$$
\frac{\mathrm{x}_{\mathrm{o}}}{\mathrm{D}}=\frac{\frac{\lambda}{2}}{\mathrm{w}}
$$

\section{Calibration}

Not applicable.

6. Accuracy

Accuracy obtainable increases with increasing film thickness.

6. 1 Greater than 0.2 micron films, $\pm 10 \%$.

6. 2 Less than 0.2 micron films, $\pm 20 \%$.

\section{Test Frequency}

This test should be performed periodically for each evaporator. 


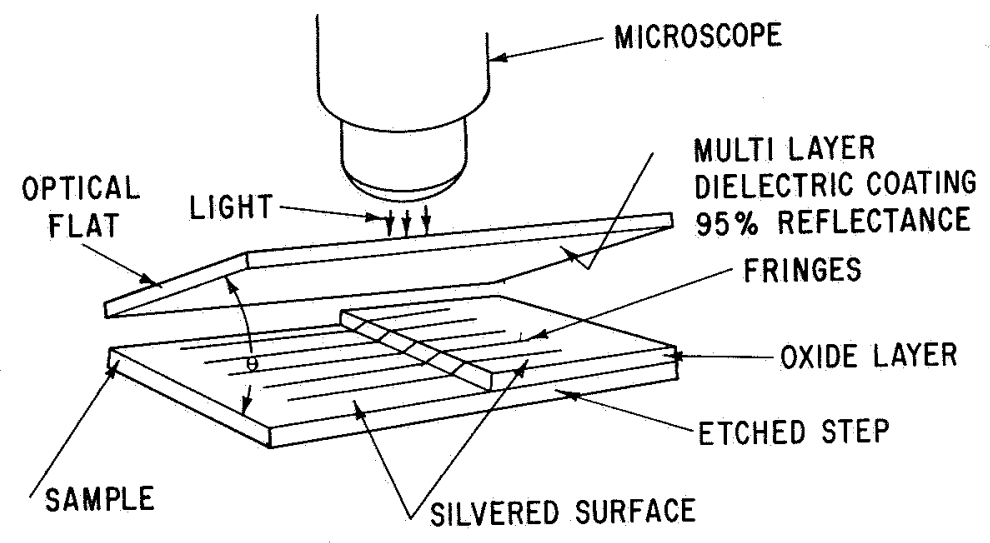

Figure 1. Tolansky Interferometer Setup 


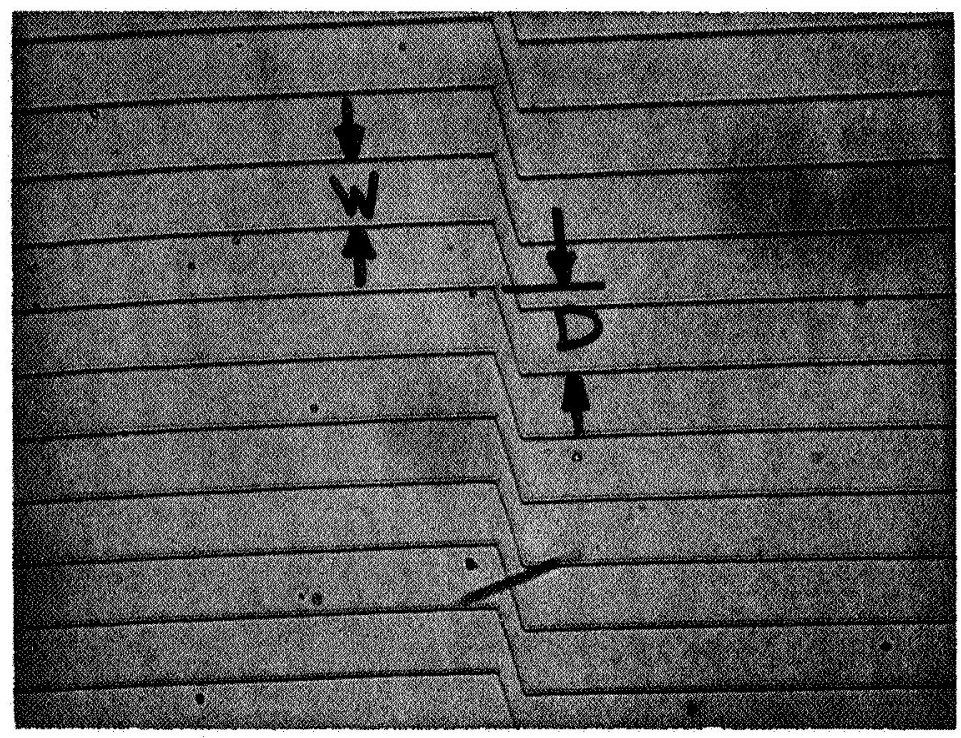

Figure 2. Film Thickness Measurement

$$
\text { Sodium, } \begin{aligned}
\lambda & =5890 \AA \\
W & =0.26 \text { inches } \\
\mathrm{X}_{0} & =\mathrm{D} \frac{\frac{\lambda}{2}}{\mathrm{~W}} \\
& =\frac{0.34 \frac{5890}{2}}{0.26} \\
& =\frac{3.4 \times 2.945 \times 10^{2}}{2.6 \times 10^{-1}} \\
& =3.92 \times 10^{-3} \\
& =3920 \AA
\end{aligned}
$$




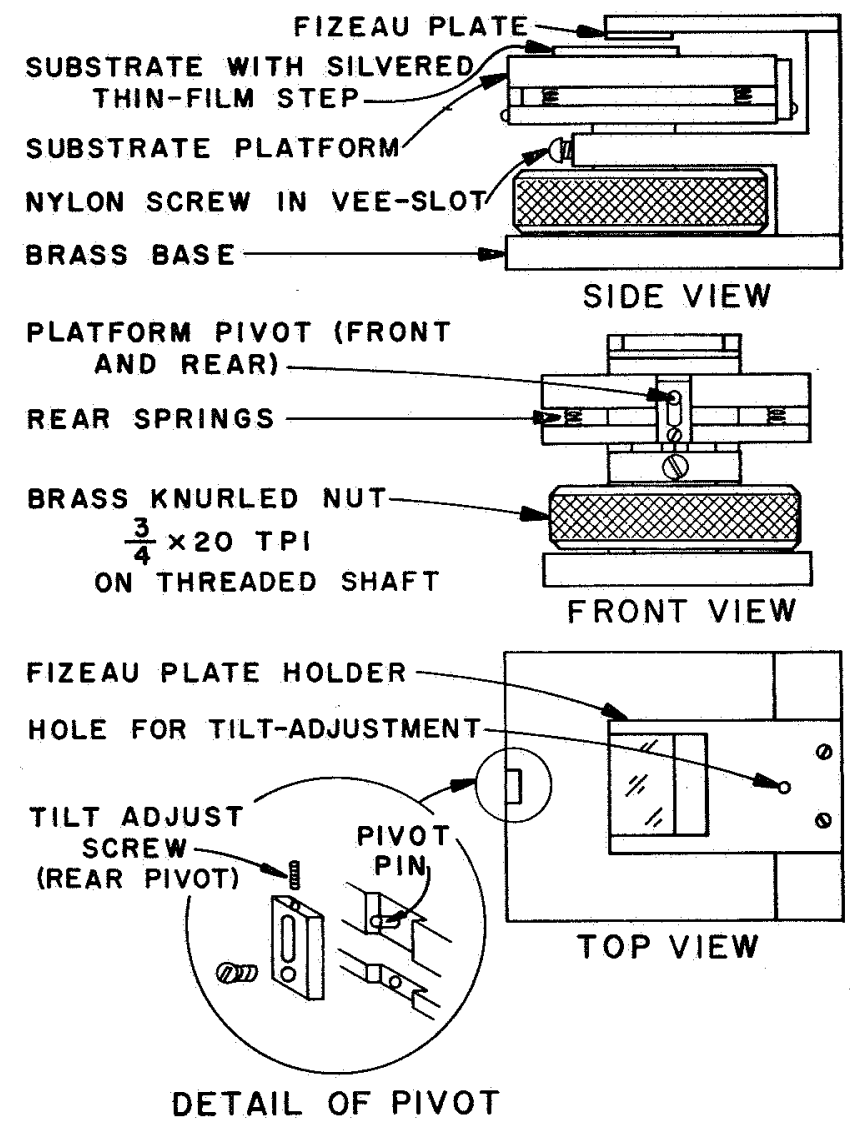

Figure 3. Interferometer Jig 


\section{Method 124 \\ Thickness of Passivating Layer \\ (Polychromatic Interference)}

1. Purpose

This method may be used to measure the thickness of thermally grown silicon dioxide. It utilizes polychromatic light and the resultant reinforcement and interference of the various colors. Shorter wave lengths are affected first, so blue would indicate a thinner film than red in the same spectral order.

\section{Apparatus}

2. 1 Metallurgica1 Microscope.

Bausen and Lomb Dynoptic, No. 31-20-68-37

(or equivalent)

2. 2 Hot plate, 6" $\times 6$ ", electric

Temco, type 1900

Corning, Pyroceram PC-35

(or equivalent)

2. 3 Polyethylene Beaker

(any source)

3. Materials

3. 1 Hydrofluoric acid, 49\%, Reagent grade.

3. 2 Trichloroethylene, Electronic grade.

3.3 Acetone, Electronic grade.

3. 4 Apiezon wax.

3. 5 Slides, glass microscope, $1^{\prime \prime} \times 3^{\prime \prime}$. 
4. Procedure

\section{1 Preparation}

4. 1. 1 Process a polished test chip through the oxidation operation(s) to be evaluated.

4. 1.2 Place a glass slide on a hot plate adjusted to a temperature slightly above the melting point of the apiezon wax $\left(150-160^{\circ} \mathrm{C}\right)$.

4. 1. 3 Place the test chip on the heated slide with polished side up.

4. 1. 4 Mask about one-fourth of the chip area with black wax.

4. 1. 5 Carefully remove the test chip from the glass slide.

4. 1.6 Etch chip in hydroflouric acid until all the oxide is removed from the unmasked area (2-3 minutes).

NOTE: When all oxide is removed, wafer will appear shiny and gray.

4. 1.7 Rinse chip in flowing water for five to ten seconds.

4. 1. 8 Remove wax from the test chip with trichloroethylene.

4. 1.9 Rinse test chip in acetone and allow to dry.

\section{2 Measurement}

4.2. 1 Place the test chip on the microscope stage with the edge of the oxide film in the field of view.

See Figure 1 .

4.2. 2 Adjust magnification until the line between oxide and bare silicon areas expands into a fringe containing one or more spectrums. See Figure 2.

4. 2. 3 Using Table I, the oxide thickness may be determined. 


\subsubsection{Above $4550 \AA$, "Fringes", spectrum boundaries, are counting as a passage from red to green. The absence of other colors will necessitate estimating thickness to the nearest one-half fringe $(910 \AA)$. Green will indicate the first one-half fringe and red the last.}

\section{Calibration}

Not applicable.

\section{Accuracy}

6. 1 From 1000-4550 , accuracy of $\pm 200 \AA$ is obtainable

6. 2 Above $4550 \AA$, accuracy is reduced to about $\pm 900 \AA$.

\section{Test Frequency}

This test should be performed on a sample from each run at each oxidation operation. 
Method 124
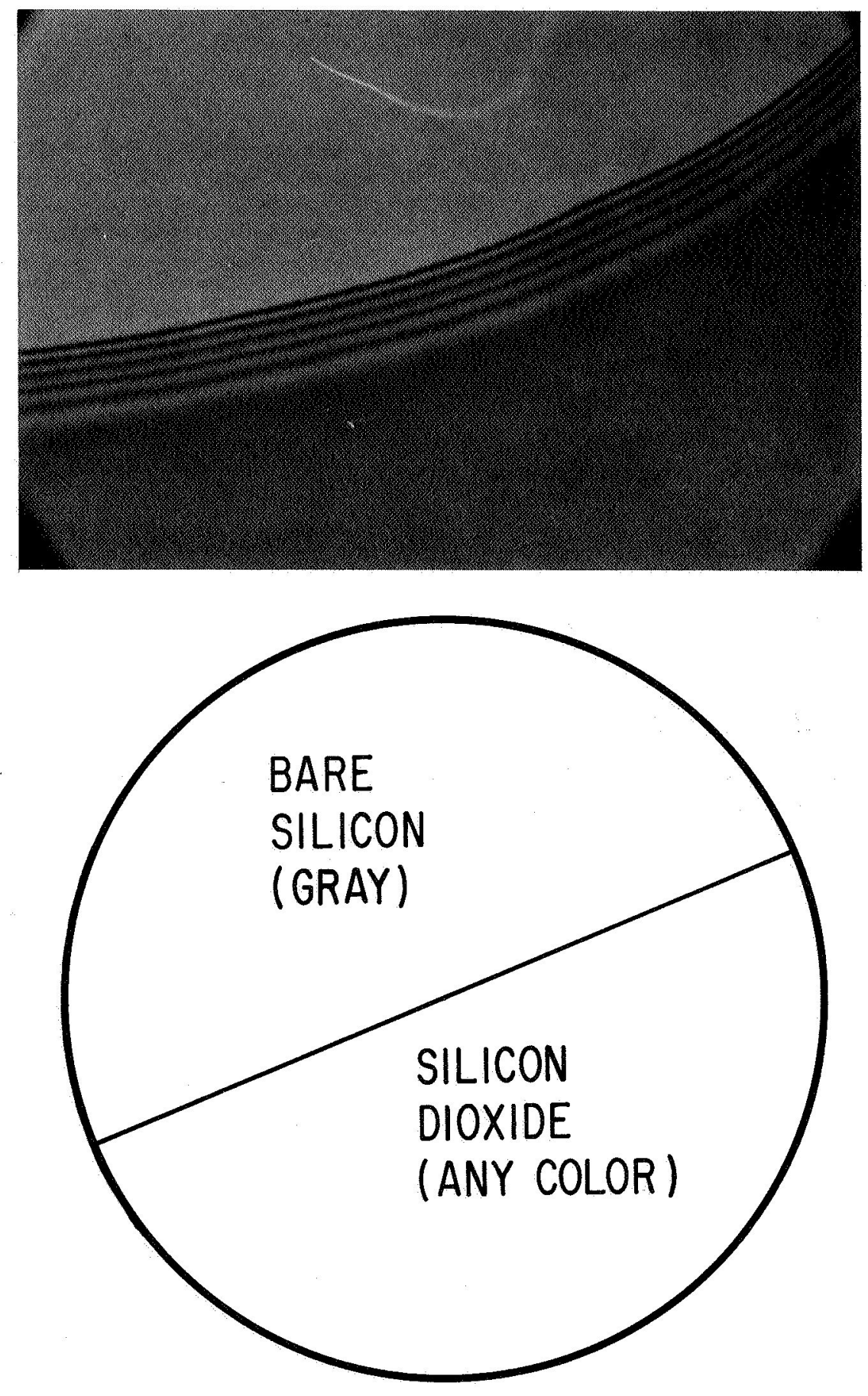

Figure 1. Oxide Step (Low Power) 
Method 124
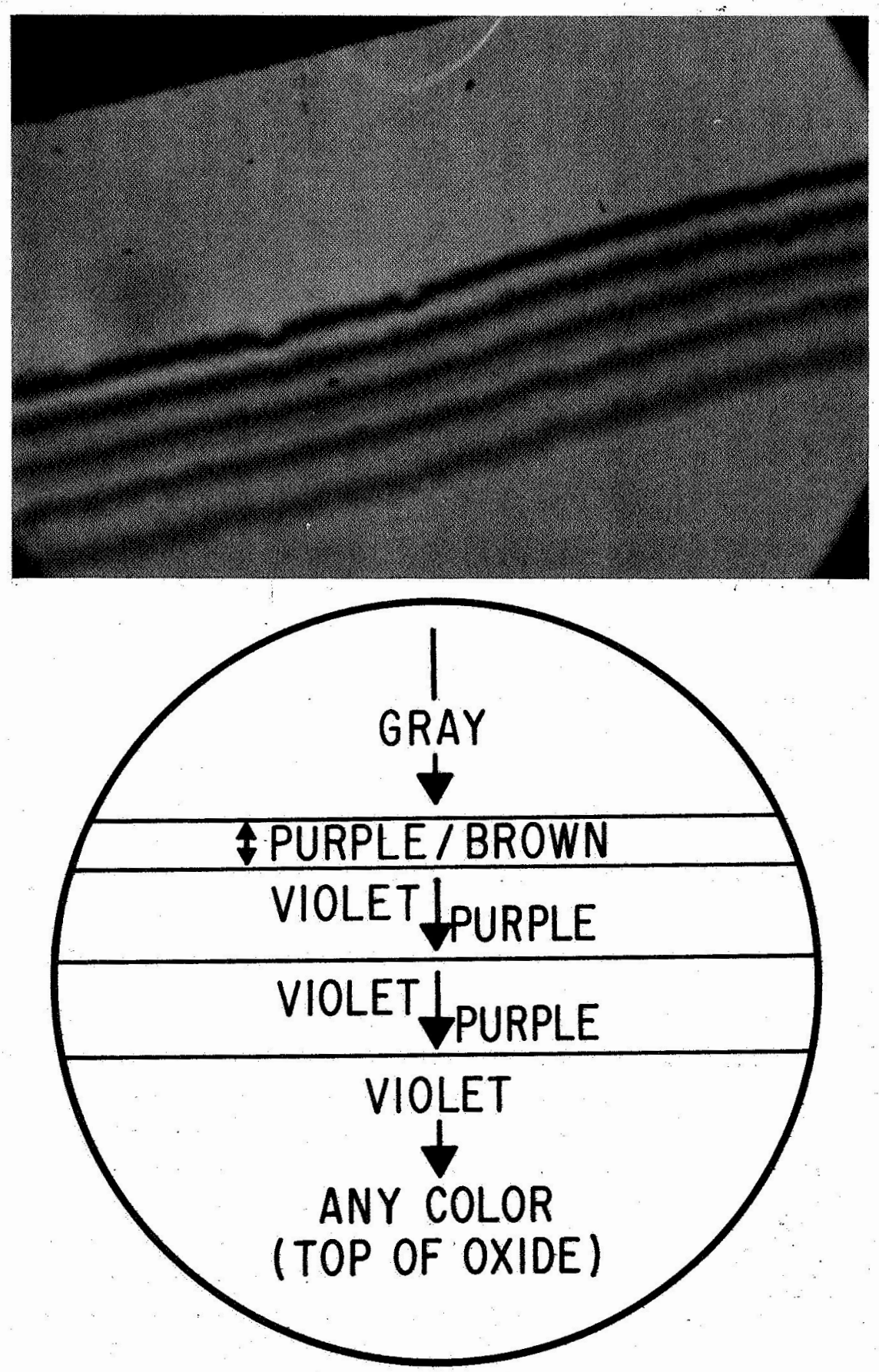

Figure 2. Oxide Step (100 x Minimum) 


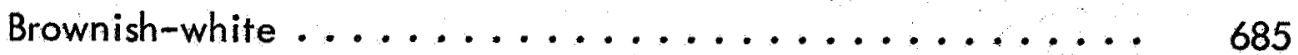

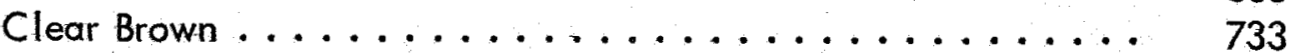

Dark Brown. . . . . . . . . . . . . . . . . . 795

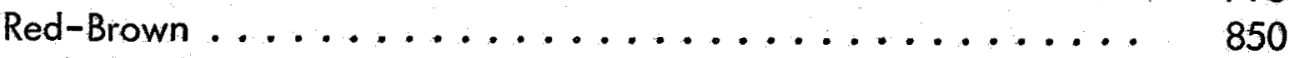

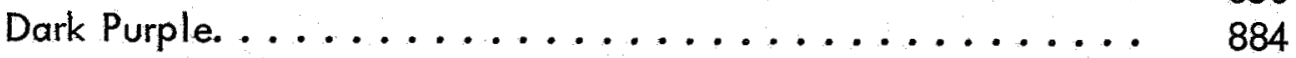

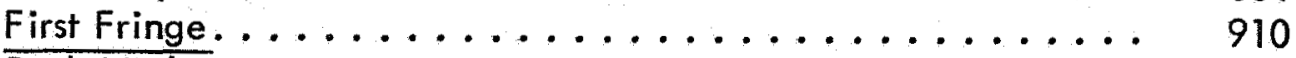

Dark Violet. . . . . . . . . . . . . . . . . . . . . . . 925

Dark Blue. . . . . . . . . . . . . . . . . . . . 959

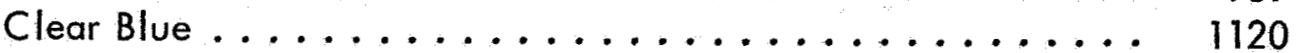

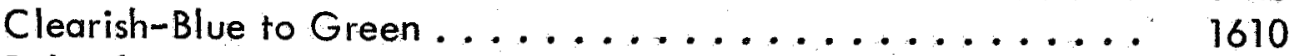

Pale Blue-Green . . . . . . . . . . . . . . . . . 1680

Pale Green. . . . . . . . . . . . . . . . . . . . 1760

Clear Yellow-Green. . . . . . . . . . . . . . . . . 1860

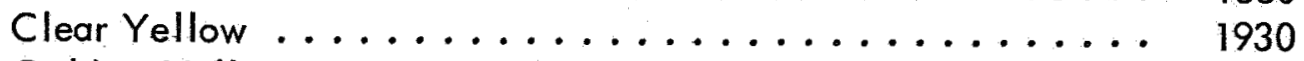

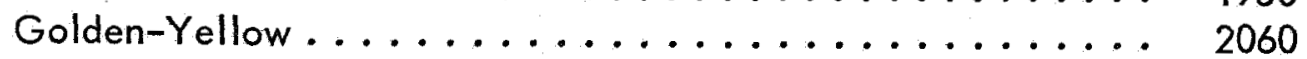

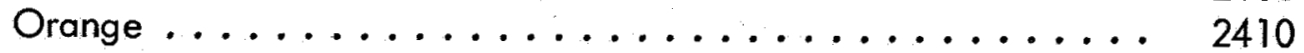

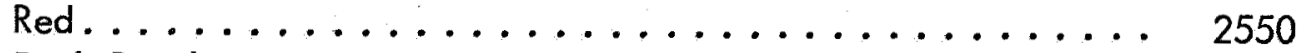

Dark Purple. . . . . . . . . . . . . . . . . . 2650

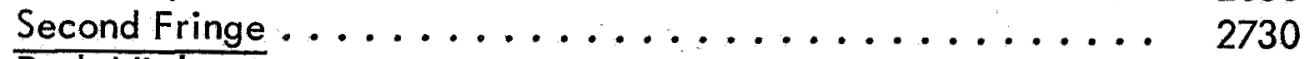

Dark Violet. . . . . . . . . . . . . . . . . . . . 2745

Dark Blue. . . . . . . . . . . . . . . . . . . . . 2779

Clear Blue ... . . . . . . . . . . . . . . . . . . . 2940

Clearish-Blue to Green . . . . . . . . . . . . . . 3430

Pale Dlue-Green ...................... . . 3500

Pale Green . . . . . . . . . . . . . . . . . . . 3580

Clear Yellow-Green..................... 3680

Clear Yellow. . . . . . . . . . . . . . . . . . 3750

Golden Yellow ....................... . . 3880

Orange ............................ 4230

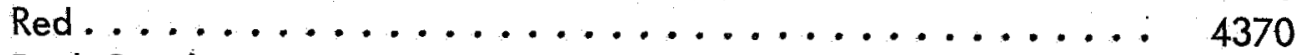

Dark Purple. . . . . . . . . . . . . . . . . . . 4470

Third Fringe ...................... 4550

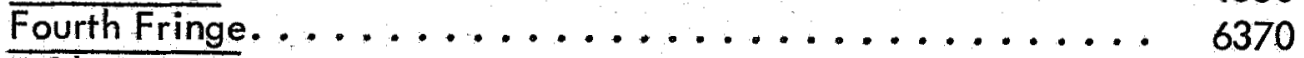

Fifth Fringe $\ldots \ldots \ldots \ldots \ldots \ldots \ldots \ldots \ldots \ldots . \ldots \ldots$

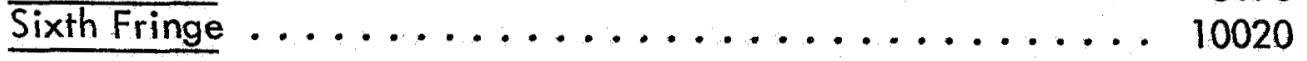




\begin{abstract}
Method 125
Thickness of Passivating Layer

(Monochromatic Interference)
\end{abstract}

1. Purpose This test procedure may be used to measure a wide range of silicon dioxide thicknesses. The interference effect on reflected rays of monochromatic light at the sloping edge of an oxide film causes a series of lines which are equally spaced. To calculate the thickness of a thin, transparent layer, backed by an opaque surface, the following equation applies:

$$
\begin{aligned}
\mathrm{d}=\frac{\mathrm{nf} \lambda}{2 \mu} & -\frac{\lambda}{4 \mu} \\
\text { where } \mathrm{d} & =\text { Layer thickness } \\
\mathrm{n} f & =\text { Number of interference fringes } \\
\lambda & =\text { Wave length of the incident light } \\
\mu & =\text { Index of refraction of the film }
\end{aligned}
$$

This method may be used on other transparent passivating layers if the proper index of refraction is used in the above equation.

\title{
2. Apparatus
}

2. 1 Metallurgical Microscope

Bausch and Lomb Dynoptic, No. 31-20-68-37

(or equivalent)

2. 2 Monochromatic Light Source

2. 2. 1 Mercury, $5461 \AA$

LaPine Scientific Company, Model 216-01

(or equivalent)

2.2.2 Sodium, $5893 \AA$ (optional)

LaPine Scientific Company, Model 215-96

(or equivalent) 
2. 3 Hot plate, 6" $\times 6$ 6", electric

Temco, Type 1900

Corning, Pyroceram PC-35

(or equivalent)

2. 4 Polyethylene Beaker

(any source)

3. Materials

3. 1 Hydroflouric acid, 49\%, Reagent grade.

3. 2 Acetic Acid, glacial, Reagent grade.

3. 3 Trichloroethylene, Electronic grade.

3. 4 Acetone, Electronic grade.

3. 5 Apiezon wax.

3. 6 Slides, glass microscope, $1^{\prime \prime} \times 3 "$.

\section{4. $\quad$ Procedure}

\section{1 Preparation}

4. 1. 1 Process a polished test chip through the oxidation operation(s) to be evaluated.

4. 1.2 Place a glass slide on a hot plate adjusted to a temperature slightly above the melting point of the apiezon wax $\left(150-160^{\circ} \mathrm{C}\right)$.

4. 1.3 Place the test chip on the heated slide with the polished side up.

4. 1. 4 Mask about one-fourth of the chip area with black wax.

4. 1. 5 Carefully remove the test chip from the glass slide.

4. 1.6 Cool the chip under running water for fifteen seconds.

4. 1.7 Immerse the chip in a prepared solution of three parts acetic acid to two parts hydroflouric acid until all unmasked oxide is removed (four to eight minutes). 
4. 1.8 Rinse chip in running water for five to ten seconds.

4.1.9 Remove wax from chip with trichloroethylene.

4. 1. 10 Rinse test chip in acetone and allow to dry.

\subsection{Measurement}

4.2.1 Place the test chip on the microscope stage with the edge of the oxide film in the field of view. See Figure 1.

4.2. 2 Adjust the magnification until all interference fringes are distinct. See Figure 2.

4. 2. 3 Count the number of fringes and determine the layer thickness. For silicon dioxide and a mercury vapor lamp, Table I may be used. For other materials or light sources, use the equation in Section 1.

NOTE: It may be convenient to photograph thick layers and use the photographic film as a permanent record.

\section{Calibration}

Not applicable.

6. Accuracy

Accuracy will be $\pm 1 / 2$ fringe, where:

$$
\text { one fringe }=\frac{\lambda}{2 \mu}
$$

\section{Test Frequency}

Each run through the passivating operation should be sampled. 
Method 125
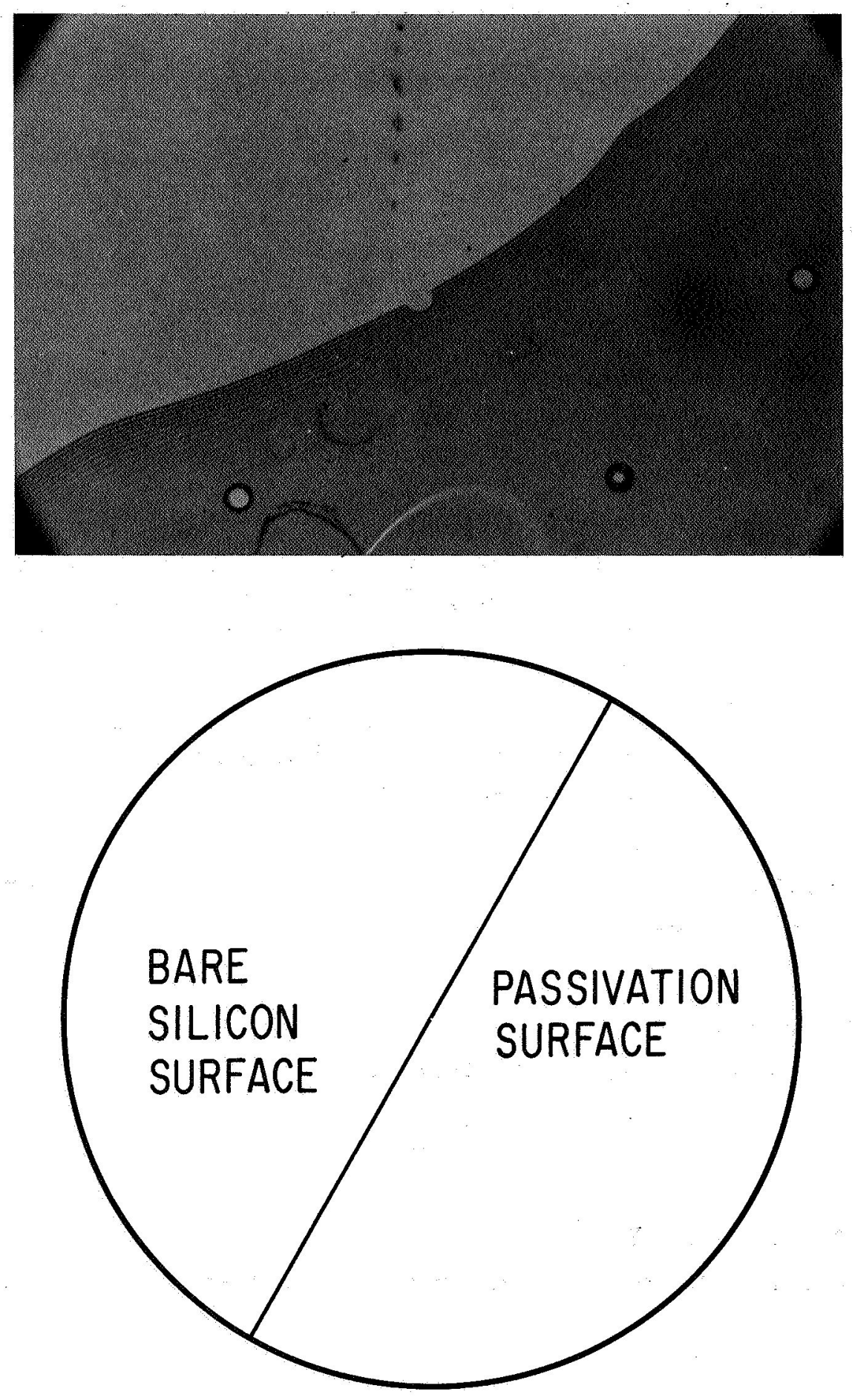

Figure 1. Oxide Step (Low Power) 
Method 125
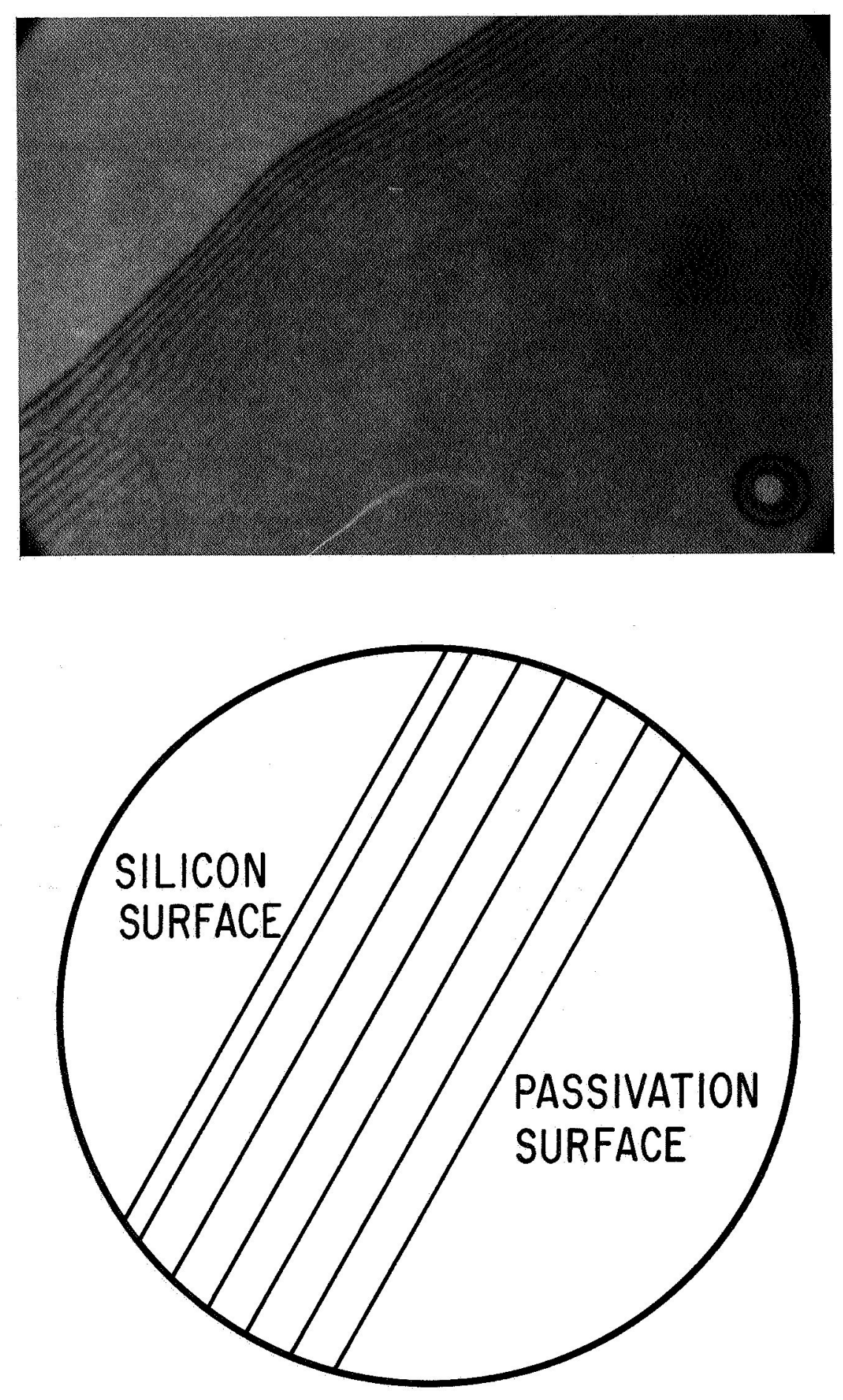

Figure 2. Oxide Step (100 x Minimum) 
Eringe Count Chart Table I

Hg Light, $\lambda=5460 \mathrm{~A}, \mathrm{n}=1.5$

\begin{tabular}{cccc}
$\begin{array}{l}\text { Number of } \\
\text { Pringes }\end{array}$ & $\begin{array}{l}\text { Thickness } \\
\text { In Microns }\end{array}$ & $\begin{array}{l}\text { Number of } \\
\text { Fringes }\end{array}$ & $\begin{array}{l}\text { Thickness } \\
\text { In Microns }\end{array}$ \\
\cline { 2 - 3 } 1 & & & \\
\cline { 2 - 3 } & .0910 & 19 & 3.3670 \\
2 & .2730 & 20 & 3.5490 \\
3 & .4550 & 21 & 3.7310 \\
4 & .6370 & 22 & 3.9130 \\
5 & .8190 & 23 & 4.0950 \\
6 & 1.0010 & 24 & 4.2770 \\
7 & 1.1830 & 25 & 4.4590 \\
8 & 1.3650 & 26 & 4.6410 \\
9 & 1.5470 & 27 & 4.8230 \\
10 & 1.7290 & 28 & 5.0050 \\
11 & 1.9110 & 29 & 5.1870 \\
12 & 2.0930 & 30 & 5.3690 \\
13 & 2.2750 & 31 & 5.5510 \\
14 & 2.4570 & 32 & 5.7330 \\
15 & 2.6390 & 33 & 5.9150 \\
16 & 2.8210 & 34 & 6.0970 \\
17 & 3.0030 & 35 & 6.2790 \\
18 & 3.1850 & &
\end{tabular}


Method 126

Stability of Passivating Layer

(C-V Curve)

1. Purpose The purpose of this test is to determine the stability of the passivating layer as indicated by a metal-oxidesemiconductor capacitor. A capacitance-voltage plot will drift with the application of temperature and bias if mobile ions are present in the passivating layer.

2. Apparatus

2. 1 X-Y Plotter

Model 520 X-Y Recorder

Honeywell

(or equivalent)

2. 2 Capacitance Meter

Model 130 L-C Meter

Tektronix

(or equivalent)

2. 3 Voltage Ramp

Variable Span 10-50 volts

Variable Rate 250-1000 milliseconds/volt

(any source)

2. 4 Single-Point Probe, with heater

(any source) Figure 1

3. Materials

3. 1 Metal Mask 0.030 inch diameter holes

3. 2 Polished substrates, N-Type

4. Procedure

General Note: Since passivating layer instability is primarily a function of mobile positive ions, cleanliness in all processes constitute a series of dependent variables. Therefore, it may be necessary to have duplicate equipment to facilitate problem location. 


\section{1 Preparation}

4. 1. 1 Furnace Evaluation

4. 1. 1. 1 Process a clean, polished substrate through the passivating furnace to be evaluated (1000-2000 layer for $\left.\mathrm{SiO}_{2}\right)$

4. 1. 1.2 Allow substrate to cool.

4. 1. 1.3 Place wafer in an aluminum evaporator on known cleanliness.

4. 1. 1. 4 Place the evaporation mask over the substrate.

4. 1.1.5 Evaporate a series of 0.030 inch dots on the test wafer. Figure 2.

4. 1. 1.6 Back etch the substrate to expose the base material.

4. 1. 1. 7 Evaporate a layer of aluminum upon the back of the sample.

4. 1. 1. 8 Alloy the aluminumized wafer.

\section{1.2 Evaporator Evaluation}

4.1.2.1 Obtain a substrate coated with a passivating layer of known stability.

4. 1.2. 2 Repeat steps 4.1.1.4 through 4.1.1.8

\section{2 Stability Measurement}

4. 2. 1 Place wafer on stage, dots up.

4.2. 2 Lower probe to dot surface.

4.2. 3 "Normalize" X-Y plotter with the pen 10 major divisions from the right side of the chart.

4.2. 4 Adjust pen to one-half major division from top of chart. 
4.2. 5 Note capacitance reading on L-C meter at normalized pen position.

4. 2.6 Start sweep on voltage ramp.

4.2. 7 Repeat 4.2. 2 to 4.2 .6 for several dots.

4. 2. 8 Apply a +10 volt bias to the first dot.

4. 2. 9 Turn heater block on and set temperature at $300^{\circ} \mathrm{C}$.

4. 2. 10 Allow wafer to remain at temperature for a minimum of 3 minutes.

4.2.11 Return wafer to room temperature.

NOTE: Once dot has been "drifted", do not allow it to remain at temperature without bias.

4.2. 12 Repeat 4.2.2 to 4.2 .7 .

4.2.13 The voltage of the first plot will indicate magnitude of $Q_{S S}$ (fixed surface state charge) and the difference in voltage between pre and post-drift plots will indicate $Q_{0}$ (mobile impurity ion charge). Figure 3.

NOTE (1): Since $Q_{S S}$ is process dependent, all pre-drift plots should be close to one another.

NOTE (2): Three characteristics may be identified for each device: pre and post drift "Flat Band Voltage" (usually $90 \%$ of normalized capacitance) and voltage at which minimum capacitance is reached.

4.2.14 Where a high degree of contamination $\left(Q_{0}\right)$ is present, the predrift plot will have a significant $Q_{0}$ component. In that case, repeat 4.2 .8 to 4.2. 12 and $Q_{0}$ will be indicated by the difference between positive and negative drift plots. Figure 4. 


\section{$\therefore \quad$ Calibration}

I-I recorder should be zeroed prior to each use by verifying zero volt pusition with roltage ramp "OFF".

c. Aceuracs

Aicuracy obtainable is \pm 0.2 volt for pre-drift voltage and \pm 0.4 voltage difference.

\section{T. Test Frequency}

Each passivation and diffusion furnace, and each evaporation should be monitored periodically. 


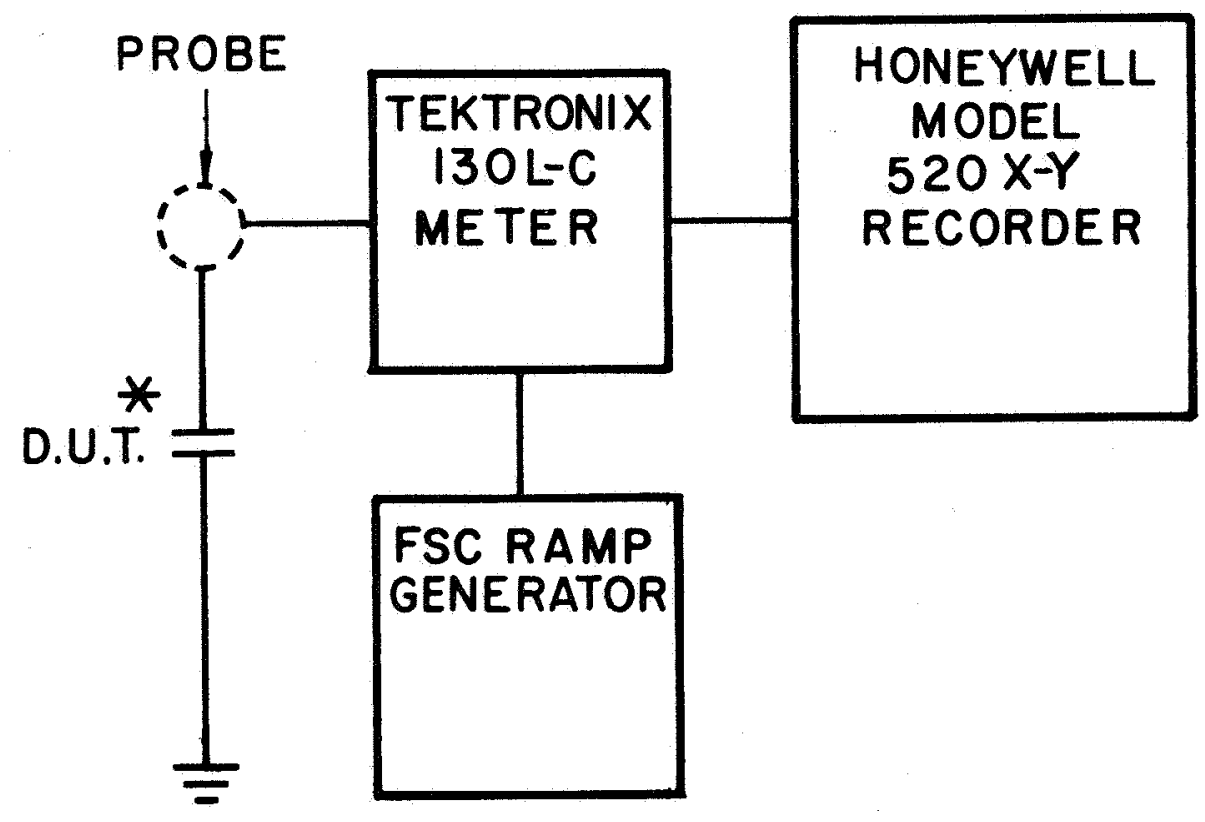

* wafer, metal pads

I. TEK 13OLC RANGE:3-300Pf $\pm 3 \%$

2. HONEYWELL $520 \pm 2 \%$

3. FSC RAMP GEN. 10-50 VOLTS $\pm 3 \%$
$250-1000$ VOLTS

Figure 1. C-V Plot Equipment 
Method 126

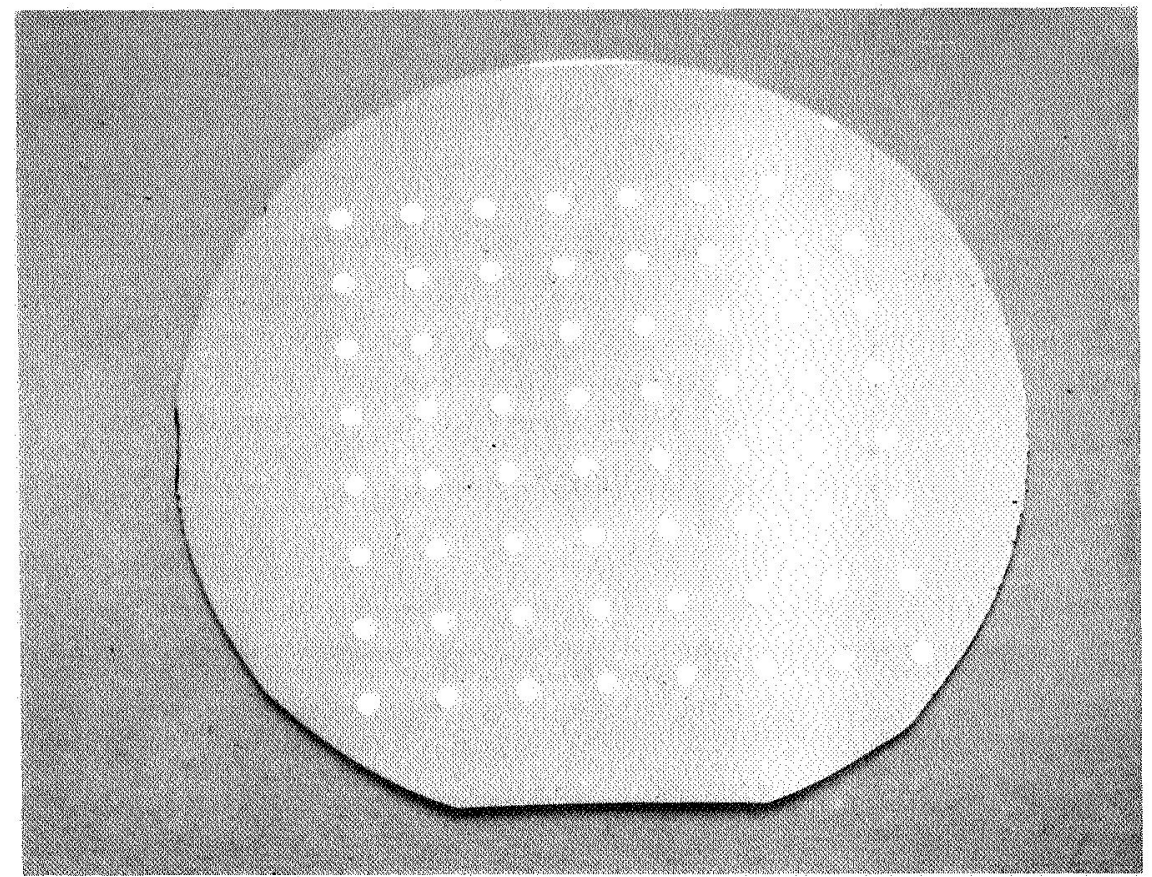

Figure 2. Test Wafer 
Method 126

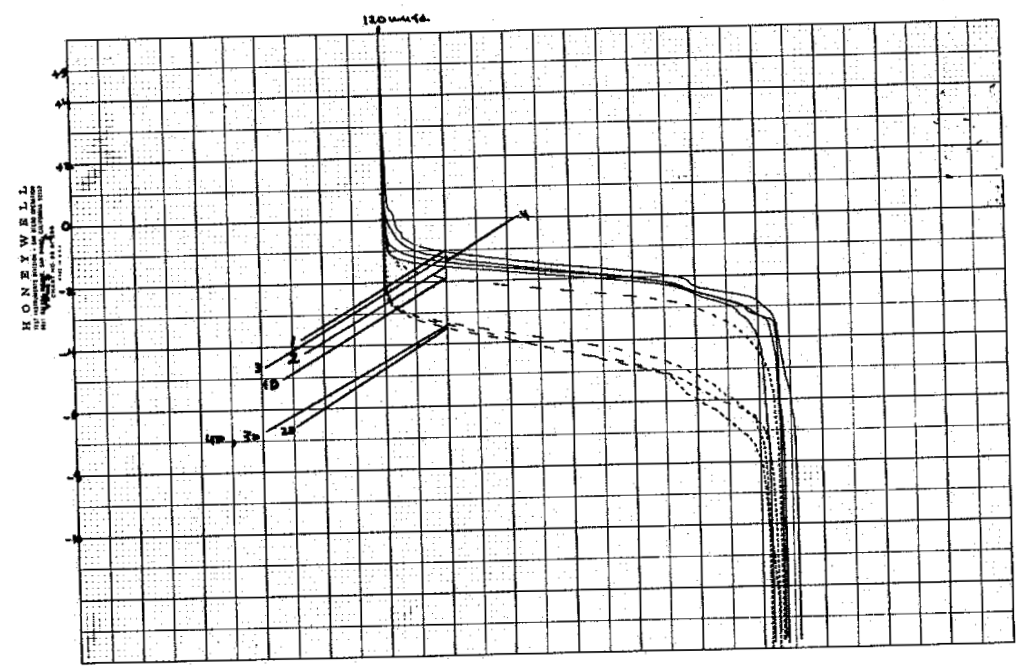

Figure 3. Positive Drift Plot 


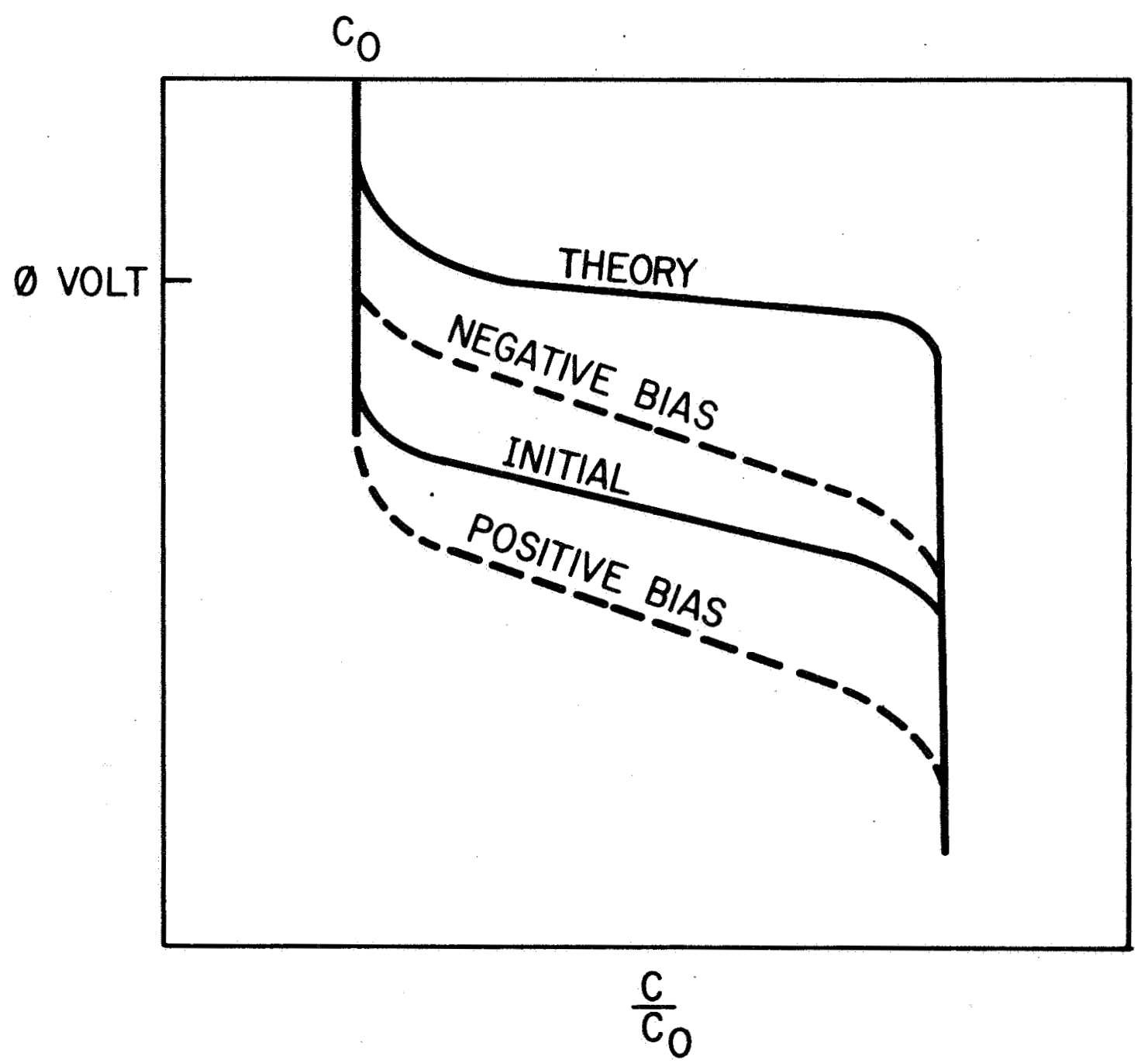

Figure 4. Comparison of Positive and Negative Drift Plots 
Method 127

\section{Pinholes in Passivating Layer}

(Silicon Etch)

1. Purpose

This method may be used to determine pinhole density in the passivating layer. Although evolved for silicon dioxide, it is applicable for other systems with the proper substitution of etchant. It utilizes the etching of silicon through the pinholes to enhance their visibility for a single oxidation or by preferentially etching doped areas in completed circuits.

\section{Apparatus}

2. 1 Metallurgical Microscope

Bausch and Lomb, Model 31-20-68-38 (DMETR)

(or equivalent)

3. Mǎ́erials

3. 1 Chromium Trioxide, Reagent grade.

3. 2 Hydrofluoric acid, Electronic grade.

3. 3 Deionized water.

4. Procedure

4. 1 Single oxidation preparation.

4. 1. 1 Mix a solution of Sirtl etch in the following proportions:
A - $100 \mathrm{ml}$ deionized water
50 grams chromium trioxide
B - $50 \mathrm{ml}$ hydrofluoric acid

NOTE: Solution may be stored after mixing. 
4. 1.2 Etch the passivated wafer a maximum of two minutes in 15 second intervals, until etched areas are visible $\left(25^{\circ} \mathrm{C}\right)$. Figure 1 .

NOTE: A one micron oxide layer will take approximately two (2) minutes; thinner film will take proportionately less time.

4. 1.3 Rinse thoroughly in deionized water and dry.

4. 2 Single oxidation measurement.

4.2. 1 Using a microscope with a grid eyepiece, count the number of pinholes in one or more squares of the grid at each of nine locations on the wafer. See Figure 2 .

4.2. 2 Calculate the pinhole density as pits per square centimeter using the following equation:

$$
\text { Density }=\frac{\text { Total No. Etched Areas }}{\text { No. Squares } \times \text { Ares of one Square }}
$$

4. 3 Completed device preparation

4.3.1 Etch all metallization from wafer.

4.3.2 Etch wafer in hydrofluoric acid until all oxide is 1 removed.

4.3.3 Rinse thoroughly in deionized water.

4.3. 4 Etch wafer for 30 seconds in the Sirtl etch of Paragraph 4.1.1

4.3. 5 Rinse thoroughly in deionized water and dry. See Figure 3.

4. 4 Device measurement.

4.4.1 Repeat 4.2.1 and 4.2.2 or count the number of pinholes per circuit at each of nine locations.

4. 4.2 Calculate the number of pinholes per circuit as follows:

$$
\text { Pinholes }=\frac{\text { Total Etched Areas }}{2}
$$


5. Calibration

Determine the area of each grid square by measuring with a stage micrometer at the magnification to be used.

6. Accuracy

Accuracy obtainable is $\pm 10 \%$.

\section{Test Frequency}

Test should be performed periodically. 
Method 127

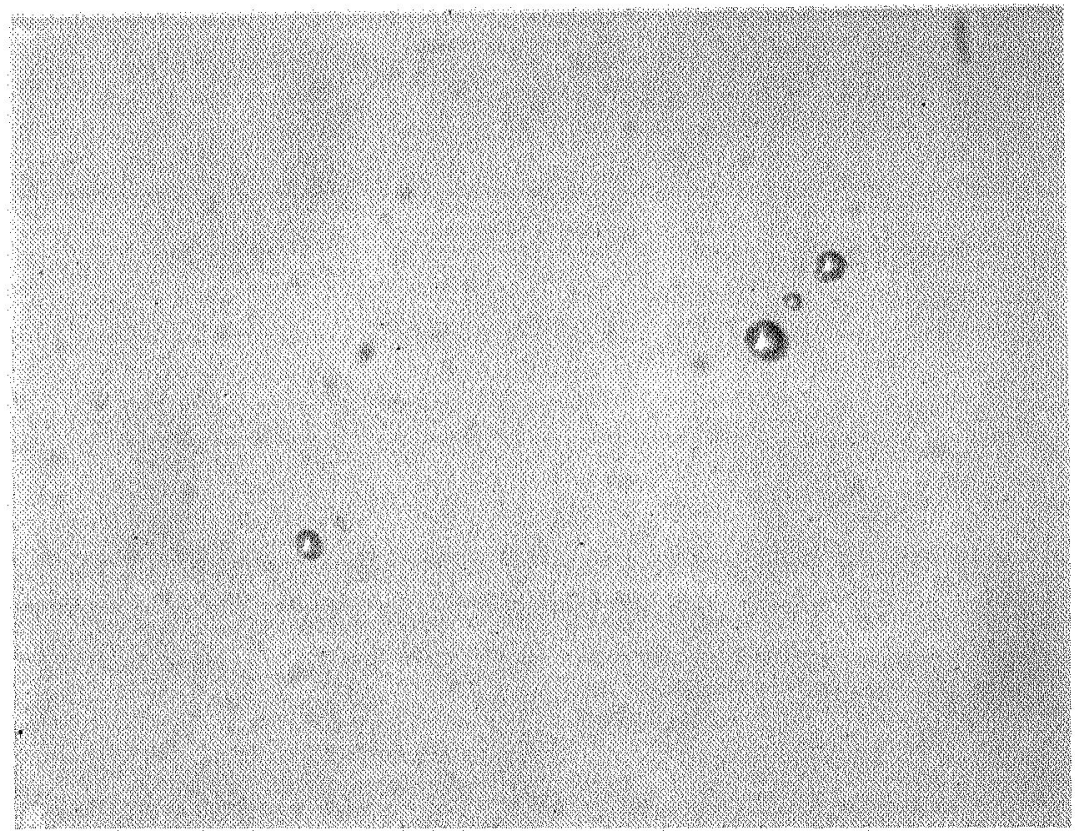

Figure 1. Pinholes Etched Through Oxide Layer 


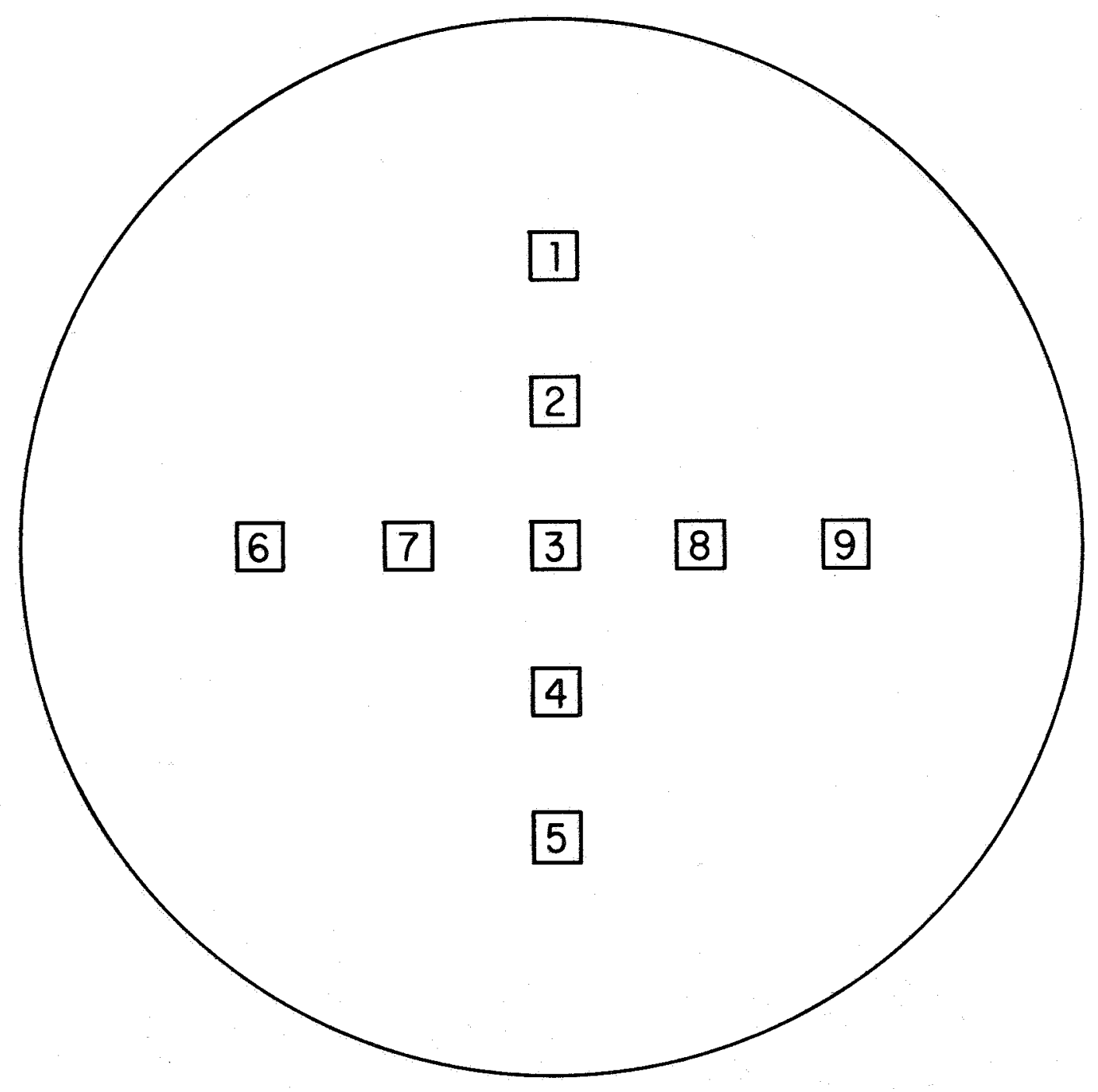

Figure 2. Sampling Pattern 
Method 127

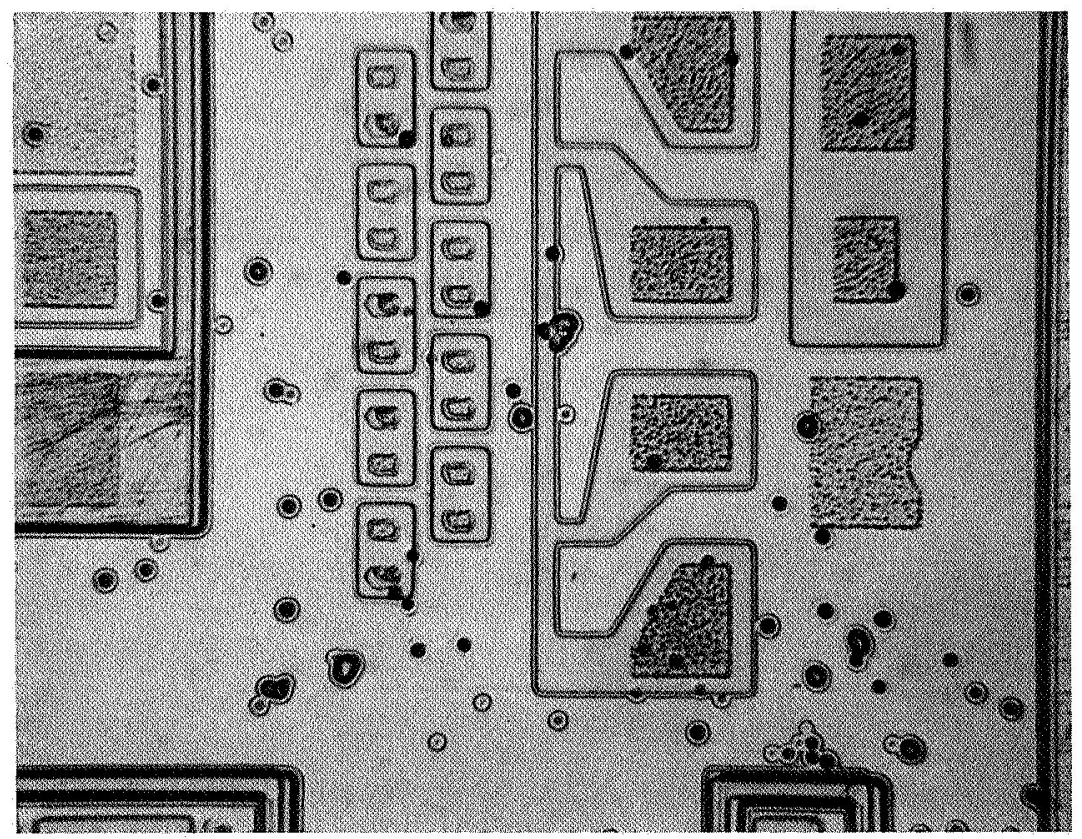

Figure 3. Pinholes in Completed Devices 
Method 128

Photoresist Process

(Minimum Line Width)

1. Purpose The purpose of this test method is to accurately measure the minimum line width etched in the passivating layer.

2. Apparatus

2. 1 Metallurgical Microscope

Bausch and Lomb.

Dynoptic No. 31-20-66-38 (DMETR)

(or equivalent)

2. 2 Watson Lens (Filar) 2x

Mode1 1014, London

(or equivalent)

3. Materials

None.

4. Procedure

4. 1 Place the wafer to be measured upon the microscope stage.

4. 2 Set the counter and vernier to "zero".

4. 3 Turn the counter to the nearest whole number where one edge of the second image just touches or does not quite reach the opposite side of the primary image. If the second image moves in any direction other than normal to the line to be measured, rotate the Watson lens until normalcy is achieved. Figure 1.

4. 4 Adjust the vernier until the two opposite sides just touch.

4. 5 Read the width of the line in whole units and tenths. Compare with calibration to obtain mils.

NOTE: Most Watson lens, used with a $40 x$ objective, will show approximately 20 units/mil. 


\section{Calibration}

The Watson lens should be calibrated by placing a $B$ and $L$ precision stage micrometer No. 31-16-99 in the apparatus and comparing the number of units per mil.

6. Accuracy

Accuracy obtainable is \pm 0.1 unit or $0.005 \mathrm{mil}$ or about $3 \%$ on a 0.00025 inch resistor.

\section{Test Frequency}

This test should be performed on a sample wafer from every photomask run. 
Method 129

Photoresist Process

(Thickness of Photoresist Film)

1. Purpose

This test method may be used to measure the thickness of a photoresist film on the substrate. It utilizes the interference of rays of monochromatic light reflected from the polished substrate surface to a sloping edge of a photoresist pattern. The following relationship is applicable:

$$
\mathrm{d}=\frac{\mathrm{nf} \lambda}{2 \mu}-\frac{\lambda}{4 \mu}
$$

where

$$
\begin{aligned}
\mathrm{d} & =\text { Layer thickness } \\
\mathrm{nf} & =\text { Number of interference fringes } \\
\lambda & =\text { Wave length of incident light } \\
\mu & =\text { Index of refraction of photoresist film. }
\end{aligned}
$$

\section{Apparatus}

2. 1 Metallurgical Microscope

Bausch and Lomb Model 31-20-68-38 (DMETR)

(or equivalent)

2. 2 Interference Filter, Transmission Type, $5500 \AA$

Bausch and Lomb No. 42-47-57

(or equivalent)

\section{3 Photoresist Station}

(process to be evaluated) 
3. Materials

3. 1 Photoresist

3. 2 Photoresist Developer

3. 3 Photomask with pattern

4. $\quad$ Procedure

\section{1 Preparation}

4. 1. 1 Obtain a clean, polished substrate which has not been passivated.

4. 1. 2 Process wafer through standard photomasking process, except mask and coated substrate shall not be pressed together and wafer should not be baked after development.

4. 2 Measurement

4.2. 1 Place the sample upon the microscope stage.

4.2. 2 Insert filter and adjust magnification to $200 \mathrm{x}$.

4.2.3 Examine the edge of any pattern with straight lines.

4. 2. 4 Starting at the substrate surface, count the number of fringes.

NOTE: A dark resist surface indicates a half fringe and a light surface is a whole fringe. See Figures 1 - 7.

4. 2.5 Apply the formula from Paragraph 1 or Table I to determine the thickness in $\AA$ units.

\section{Calibration}

Not applicable. 
6. Accuracy

Accuracy obtainable should be $\pm 1 / 2$ fringe, or $910 \AA$.

7. Test Frequency

This test should be performed periodically on each process photoresist combination. 


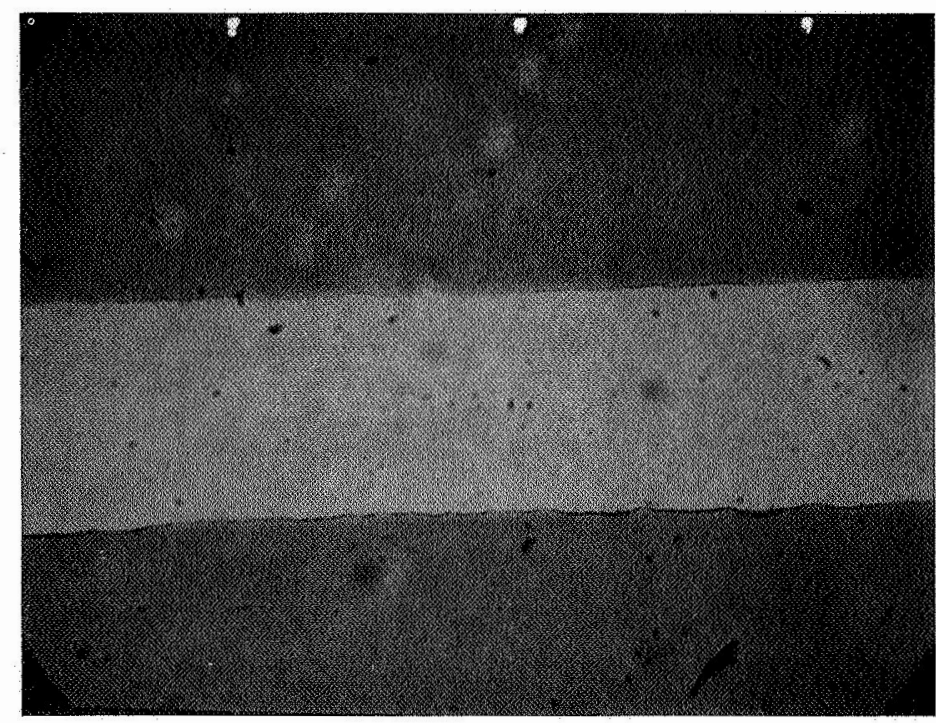

Figure 1. One-half Fringe KMER Coating 200X

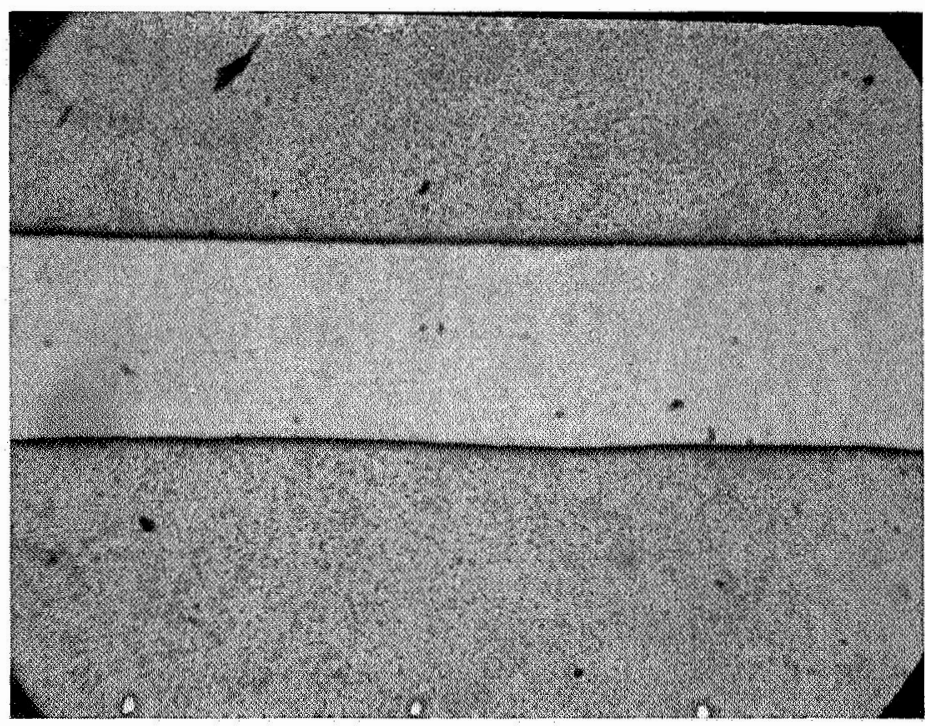

Figure 2. One Fringe KMER Coating 200X 
Method 129

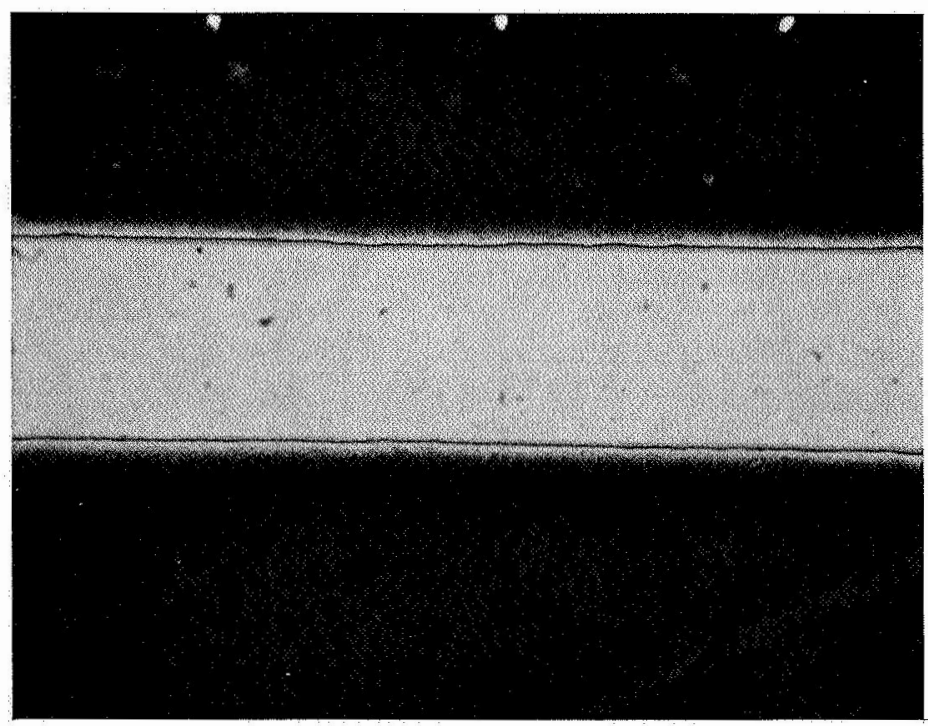

Figure 3. One and one-half Fringes KMER Coating $200 \mathrm{X}$

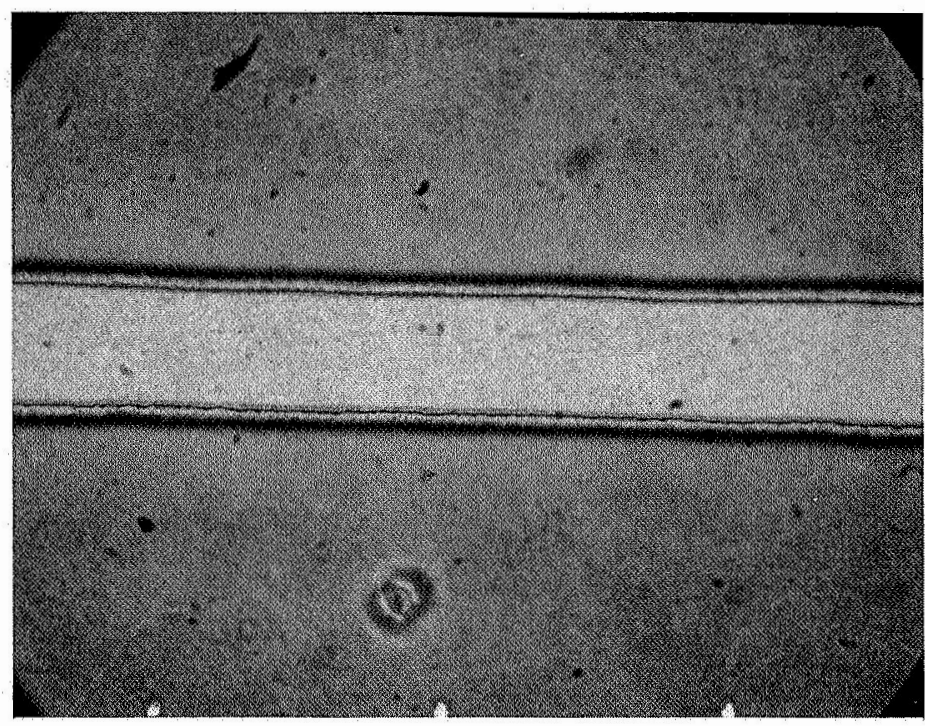

Figure 4. Slightly Less Than Two Fringes KMER 200X 


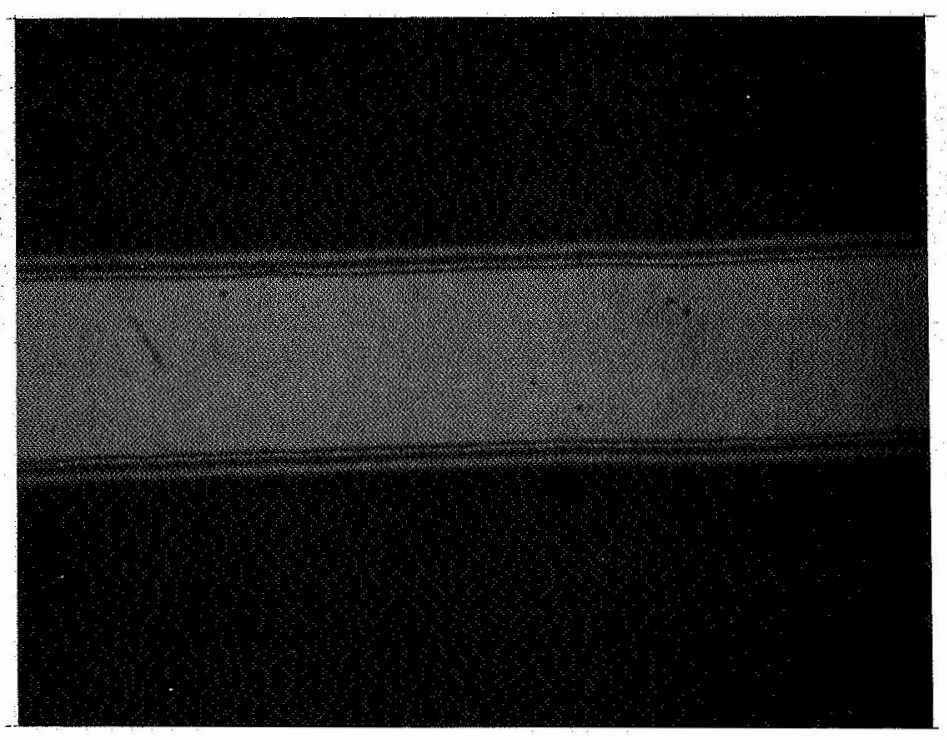

Figure 5. Two and one-half Fringes 200X

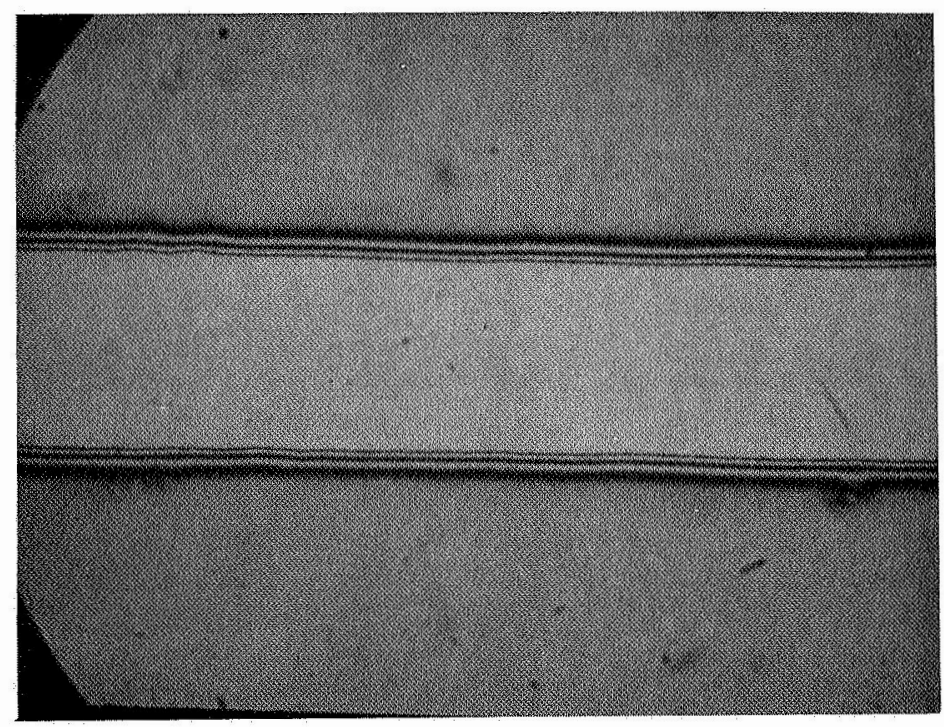

Figure 6. Three Fringes $200 \mathrm{X}$ 
Method 129

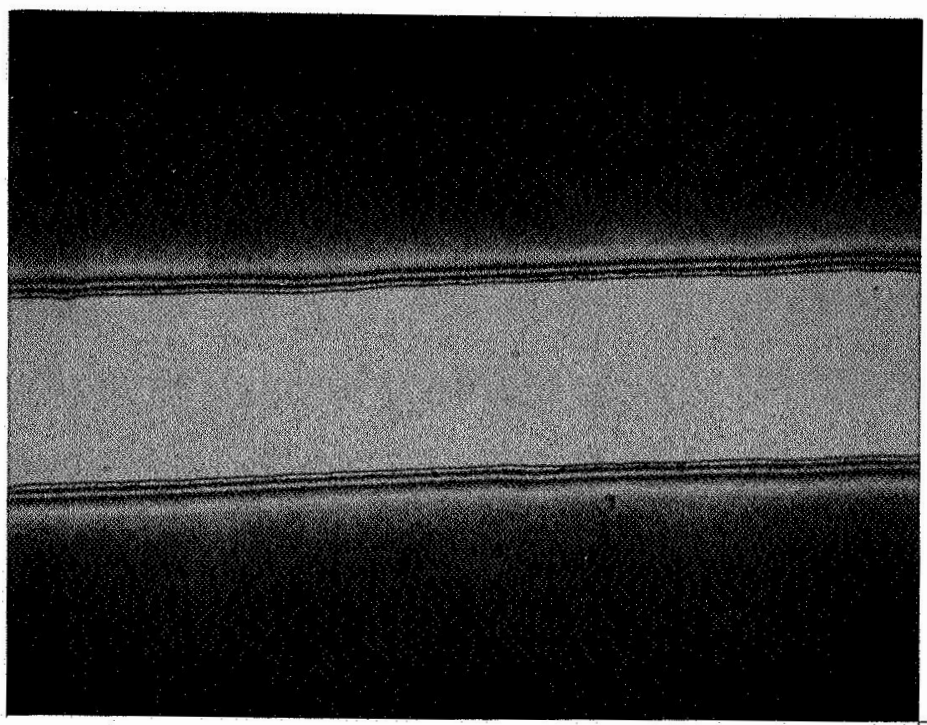

Figure 7. Three and one-half Fringes $200 \mathrm{X}$ 


\section{Fringe Count Chart Table I \\ Hg LIght, $\lambda=5460 \mathrm{~A}, \mathrm{n}=1.5$}

\begin{tabular}{cc}
$\begin{array}{c}\text { Number of } \\
\text { Pringes }\end{array}$ & $\begin{array}{l}\text { Thickness } \\
\text { In Microns }\end{array}$ \\
\cline { 1 - 1 } 1 & \\
2 & .0910 \\
3 & .2730 \\
4 & .4550 \\
5 & .6370 \\
6 & .8190 \\
7 & 1.0010 \\
8 & 1.1830 \\
9 & 1.3650 \\
10 & 1.5470 \\
11 & 1.7290 \\
12 & 1.9110 \\
13 & 2.0930 \\
14 & 2.2750 \\
15 & 2.4570 \\
16 & 2.6390 \\
17 & 2.8210 \\
18 & 3.0030 \\
& 3.1850
\end{tabular}

\begin{tabular}{ll} 
Number of & Thickness \\
Exinges & in Microns \\
\hline
\end{tabular}

3.3670

3.5490

3.7310

3.9130

4.0950

4.2770

4.4590

25

26

27

28

29

30

31

32

33

34

35

4.6410

4.8230

5.0050

5.1870

5.3690

5.5510

5.7330

5.9150

6.0970

6.2790 
Method 130

Photoresist Process

(Pinholes in Film)

1. Purpose The purpose of this method is to determine the density of pinholes in the photoresist film. It is accomplished by measuring the number of resistcaused pinholes in the passivating layer of a polished substrate.

2. Apparatus

2. 1 Metallurgical Microscope

Bausch and Lomb, Mode1 31-20-68-38 (DMETR)

(or equivalent)

2. 2 Photoresist Station

(process to be evaluated)

3. Materials

3. 1 Passivation Etch

3. 2 Photoresist

3. 3 Photoresist Developer

3. 4 Photoresist Stripping Solution

3. 5 Photomask, $0.025 \times 0.025$ inch island.

4. Procedure

4. 1 Preparation

4. 1. 1 Obtain a polished, passivated substrate.

4. 1.2 Process wafer through standard photomasking process using the mask called out in 3.5

4. 1.3 Etch grid pattern through passivating layer. 
4. 1. 4 Strip photoresist from wafer.

4. 1. 5 Rinse wafer in deionized water and dry.

4. 2 Measurement

4.2. 1 Place sample wafer on microscope stage.

4.2.2 Adjust magnification to $200 \mathrm{X}$.

4.2. 3 Count pinholes in sixteen random squares.

NOTE: A pinhole is defined as a ring of light or dark field, or a hole with a flat, silver-grey base in light field. Fig. 1

4.2. 4 Divide total number of pinholes found in 4.2 .3 by 100 for the pinhole density per square inch.

\section{Calibration}

Not applicable.

6. Accuracy

Accuracy obtainable should be at least $\pm 10 \%$ of pinhole count.

7. Test Frequency

This test should be performed periodically on each processphotoresist combination. 


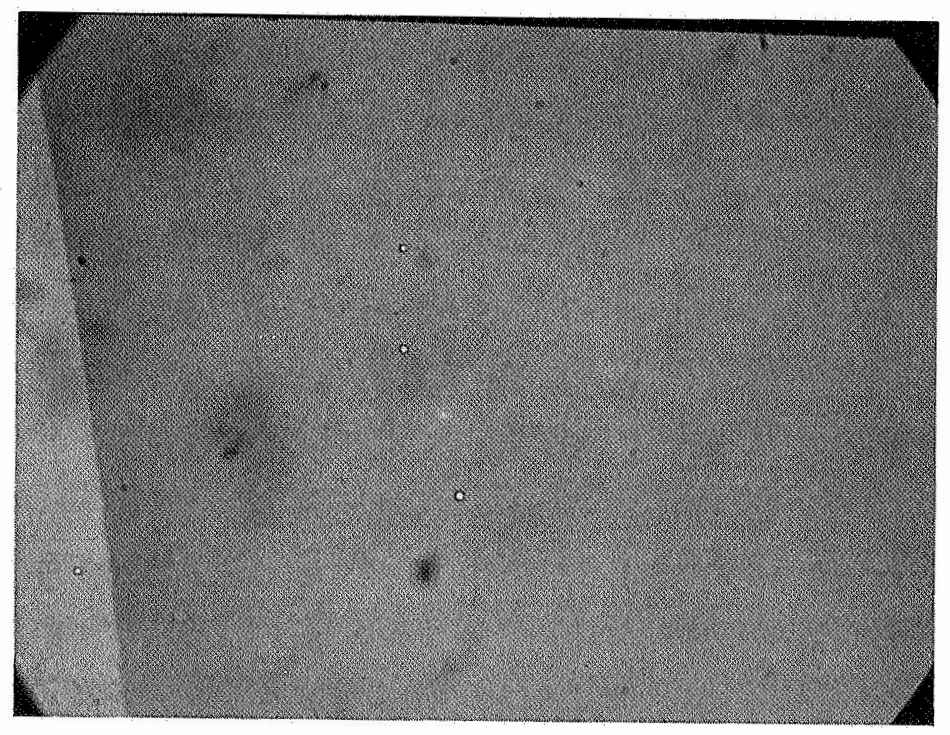

Light Field

Dark Field

Figure 1. Oxide Pinholes 
Method 131

\section{Photoresist Process}

(Light Intensity)

1. Purpose This method may be used to obtain reproducible light intensity in the photoresist process. A stable radiometer is used to calibrate a mercury light source which is the source of calibration for any small-area production lightmeter. The wavelength of interest is the $4050 \AA$ mercury line.

\section{Apparatus}

2. 1 Mercury Source Lamp, Adjustable Mirror and Focus (any source)

2. 2 Plywood Screen, $3^{\prime} \times 4^{\prime} \times 1 / 2^{\prime}$ Dull Black with $31 / 2 " \times 51 / 2 "$ Baffled Aperture

2. 3 Water Filter for Paragraph 2.2

2. 4 Narrow-Bandpass Filter Combination

Corning, Color Specification 5-62

(or equivalent)

2. 5 Aluminum Filter Holder, Black, 10" $\times 10^{\prime \prime} \times 1 / 8^{\prime \prime}$ with $11 / 2 "$ Circular Baffled Aperture

2. 6 Shutter

(any source)

2. 7 Wideband Calibrated Radiometer

Eppley Thermopile No. 6965

(or equivalent) 

2.8 Window, $\mathrm{BaF}_{2}$
Eppley
(or equivalent)

2. 9 Window, Quartz

GE Type 101

(or equivalent)

2. 10 Lightmeter, Small Area

(any source)

\section{Materials}

None.

4. Procedure

4. 1 Turn out the exposing light source and allow it to warm up.

4. 2 Place the light meter sensing head on the wafer stage. See Figure 1.

4. 3 Note the intensity as indicated by the meter.

4. 4 Scan the wafer stage, noting uniformity of intensity.

4. 5 Adjust exposure optical system to obtain maximum uniformity.

4. 6 With the sensing head in the center of the stage, adjust exposure intensity to the desired level.

\section{Calibration}

Calibrate the reference light source and production lightmeter as follows:

5. 1 Set up the optical bench as shown in Figure 2.

5.2. Filter sequence from source to detector should be either yellow-blue-violet, or blue-yellow-violet. 
5. 3 Turn on source light and allow it to warm up.

5. 4 Adjust source mirror and focus to deliver less than $10 \%$ intensity variation over a $2 \mathrm{~cm}$. diameter area on the optical axis.

5. 5 Adjust intensity to the reference level.

5. 6 Swing thermopile off axis to expose device under test.

5. 7 Note reading of test device.

5. 8 Adjust zero of test device until its current reading agrees with its original reading at the calibration intensity.

6. Accuracy

Accuracy obtainable depends upon manufacturer.

7. Test Frequency

Production exposure equipment should be adjusted daily. Light meter should be calibrated periodically. 
Method 131
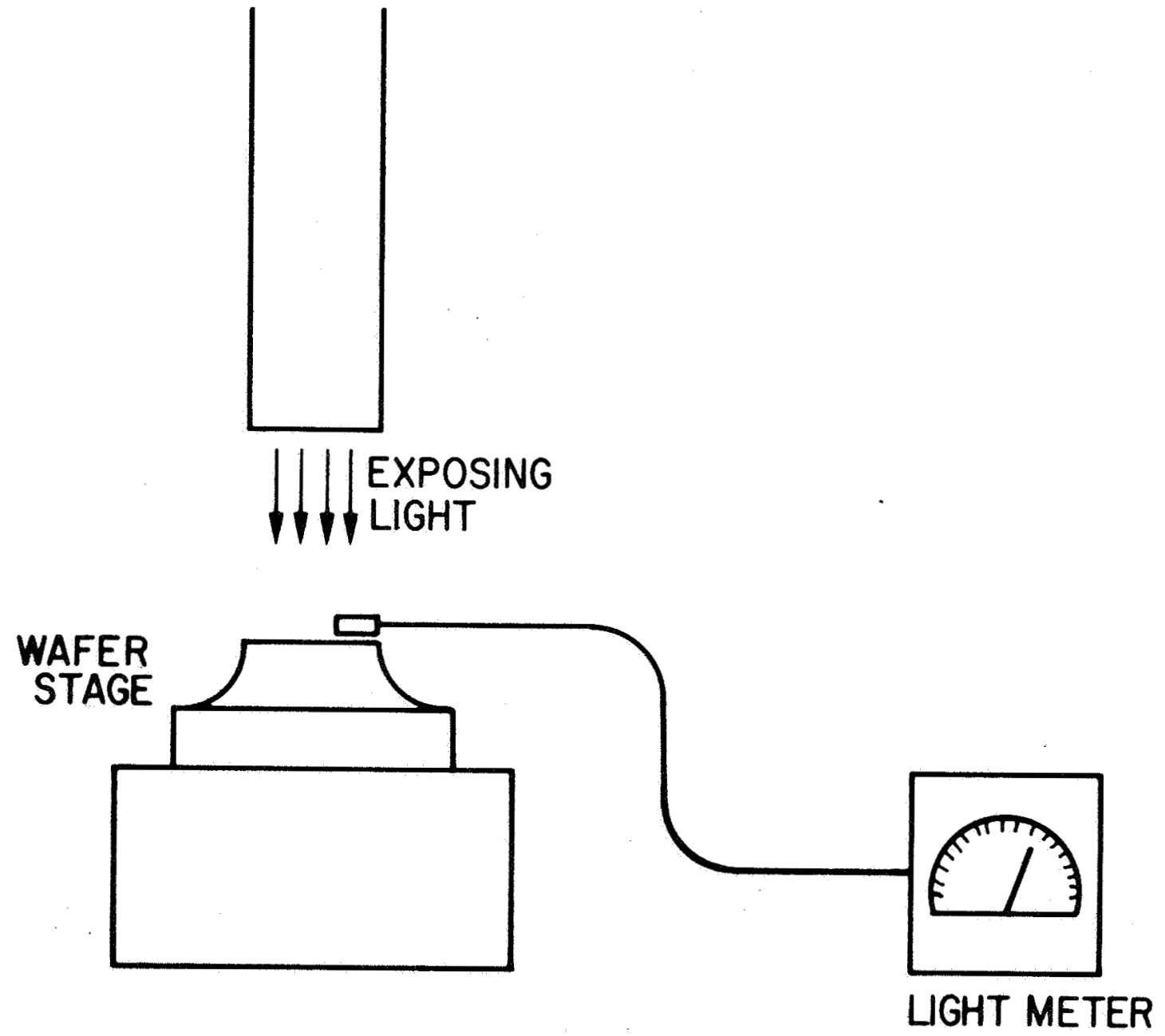

Figure 1. Exposing Light Calibration 
Method 131

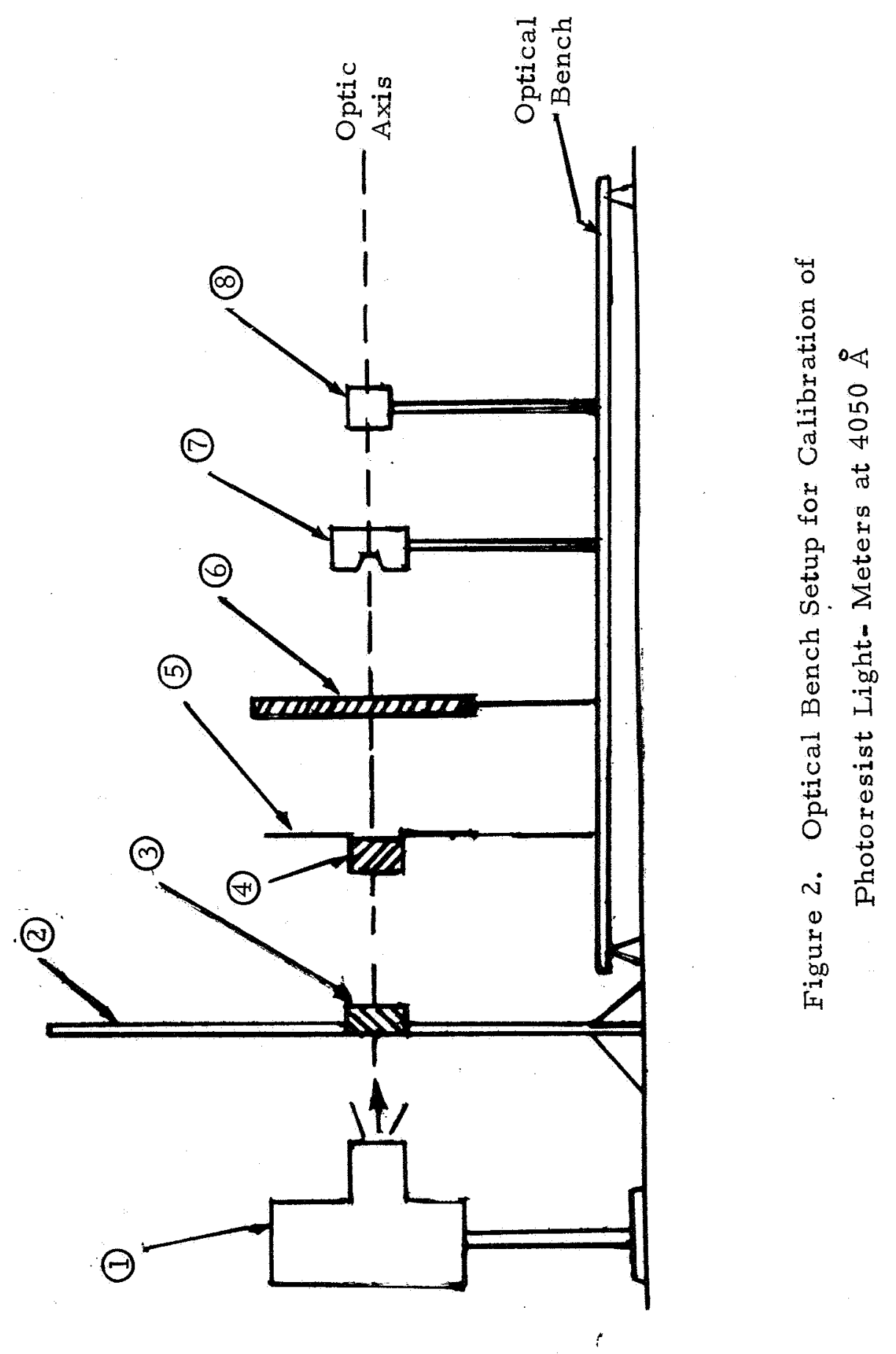


Method 132

Photoresist Process

(Spinner RPM)

1. Purpose This test may be used to determine the speed of a spinner used to apply a thin, uniform film of photoresist. It utilizes a stroboscope to synchronize with the spinner, then its speed is measured directly by the flashing rate required to hold the image stationary.

2. Apparatus

2. 1 Stroboscope

Type 1531.A Strobotac

General Radio

(or equivalent)

3. Materials

None.

4. Procedure

4. 1 Open the stroboscope case and plug the power connector into a receptacle.

4. 2 Pivot the reflector assembly to an upright position.

4. 3 Turn the power switch on and allow 10 seconds for warm up.

4. 4. Aim the light beam at the spinner to be calibrated.

4. 5 Turn the spinner on and adjust control to approximately the speed desired.

4. 6 Select the speed range which is applicable.

4. 7 Rotate the RPM control until the spinner image stops. This is the speed of the spinner. 
NOTE: If multiple images occur, the stroboscope is set at an even multiple of the fundamental speed and should be slowed until only one image is visible.

4. 8 Adjust the stroboscope to the spinner speed desired.

4.9 Increase or decrease the spinner speed until one stationary image is again obtained.

4. 10 Record the spinner control setting and the delivered RPM.

\section{Calibration}

Adjust the calibration for any given power-line frequency as follows:

5. 1 Allow a 10 minute warm-up.

5. 2 Turn the range switch to 670-1410 RPM:

5. 3 Set the RPM dial to 3600 RPM (for 60 cycle service).

5. 4 Adjust the screw-driver control marked "HIGH CAL" until the on - off pattern of the neon indicator lamp stops or nearly stops.

5. 5 Set the RPM dial to 900 RPM (for 60 cycle service) and repeat 5. 4 for the "LOW CAL" screw-driver adjustment.

5. 6 Return RPM dial to 3600 RPM and repeat 5. 4.

6. Accuracy

Accuracy obtainable after calibration is $\pm 1 \%$.

7. Test Frequency

Photoresist spinners should be calibrated periodically per this or equivalent methods. 


\author{
Method 133 \\ Junction Formation (Depth) \\ (Monochromatic Interference)
}

1. Purpose The purpose of this test is to measure the depth of diffused junctions. It employs the interference of monochromatic light reflected between the beveled surface of the applicable structure and a thin, flat glas s slide.

2. Apparatus.

2. 1 Metallurgical microscope

Metalstar trinocular body AO No. 2200T-4

American Optical Company

(or equivalent)

2. 2 Polaroid camera AO No. 1052A

American Optical Company

(or equivalent)

2. 3 Monochromatic light source

2.3. 1 Mercury, $5461 \AA$, green filter

LaPine Scientific Co. Model 216-01

(or equivalent)

2.3.2 Sodium, $5893 \AA$ (optional)

LaPine Scientific Co. Model 215-96

(or equivalent)

2. 4 High intensity light fixture

Sun Gun II

Eastman Kodak

(or equivalent)

2. 5 High intensity bulb

3400K movie lamp type DWY

Sylvania Electric

(or equivalent) 
2. 6 Hot plate, 6" $\times 6$ 6", Electric

Temco, type 1900

Corning, Pyroceram, PC-35

(or equivalent)

2. 7 Polyethylene beaker, (any source)

2. 8 Glass plate, $6^{\prime \prime} \times 6^{\prime \prime}$, (any source)

3. Materials

3. 1 Hydrofluric acid, 49\%, reagent grade

3. 2 Nitric acid, concentrated, reagent grade

3. 3 Acetic acid, glacial, reagent grade

3. 4 Trichloroethylene, electronic grade.

3. 5 Acetone, electronic grade

3. 6 Apiezon wax

3. 7 Polishing compound, 1 micron, Linde "A"

3. 8 Cover glass, microscope $24 \times 30 \mathrm{~mm}$, No. $11 / 2$ thinness Corning or equivalent

4. Procedure

4. 1 Preparation

4. 1. 1 Place an angle section block, (Figure 1), on a hot plate adjusted to a temperature slightly above the melting point of apiezon wax $\left(150-160^{\circ} \mathrm{C}\right)$.

4. 1.2 Place a small amount of wax in the sample niche.

4. 1.3 Insert the structure to be sectioned into the sample niche, pattern up.

Note: For ease of measurement, passivating layer should be removed. 
4. 1. 4 With tweezers or applicators, orient the junction over the edge of the bevel and seat the sample.

4. 1. 5 Remove the block from the hot plate and allow to cool.

4. 1.6 Mix a slurry of Linde "A" and water.

4. 1. 7 Place a quantity of the slurry on a flat glass plate.

4. 1.8 Invert the angle block and place the $5^{\circ}$ bevel against the glass plate.

4. 1.9 Adding Linde "A" as necessary, polish the specimen to a microscopically smooth finish.

4. 1. 10 Mix a junction stain of the following composition:

$$
1 \mathrm{HF}: 3 \mathrm{HNO}_{3}: 20 \mathrm{HAc}
$$

4. 1. 11 Rinse the mounted specimen thoroughly in deionized water and blow dry with a nitrogen gun.

4. 1. 12 Place the cleaned specimen in the staining apparatus, (Figure 2), or beneath a "Sun Gun".

4. 1. 13 Illuminate the specimen for 15 seconds.

4. 1. 14 Prior to turning off the light, place the $1: 3: 20$ staining solution on the specimen, using a cottontipped applicator.

4. 1.15 Allow the stain to remain on the sample for 5-10 seconds.

4.1.16 Turn off the light and quickly rinse in DI water.

4. 1. 17 Examine the specimen for adequate junction delineation. Additional polishing or staining may be necessary.

4. 1. 18 Return mounted specimen to the hot plate until the wax melts.

4. 1. 19 Clean the dismounted specimen with TCE and acetone. Allow to dry. 


\section{2 Measurement}

4. 2. 1 Place the specimen on the microscope stage with a microscope cover glass over the junction. See Figure 3 .

4.2. 2 Adjust magnification and focus until the junction (s) of interest and interference fringes are clear.

4.2.3 Take a photo micrograph.

4.2. 4 Starting with the top surface, count the number of fringes to the junction, or just short of it if the junction lies between fringes.

4.2. 5 If the junction is not on a whole fringe, measure the distance between fringes, $W$, with a precision rule.

4. 2.6 Measure the distance from the last fringe to the junction, D.

4.2. 7 Calculate the junction depth, $x_{j}$, from the
following equation:

$$
\begin{aligned}
& x_{j}=n_{f}\left(\frac{\lambda}{2}\right)+\frac{D}{W}\left(\frac{\lambda}{2}\right) \\
& \text { where } n_{f}=\text { number of fringes } \\
& \lambda=\text { wave length of incident light }
\end{aligned}
$$

See Figure 4.

\section{Calibration}

Not applicable.

\section{Accuracy}

Accuracy obtainable is \pm 0.1 micron.

\section{Test Frequency}

This test should be performed on a sample from each run after each diffusion. 


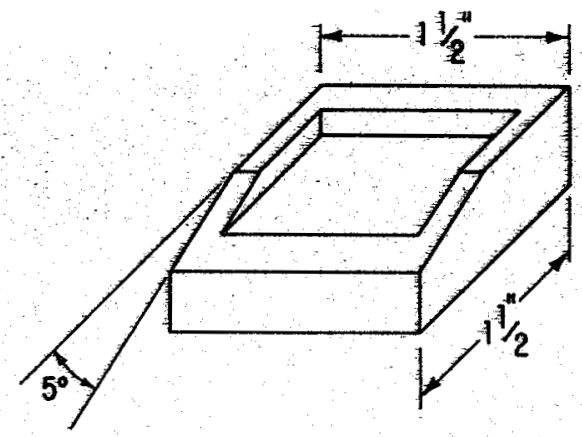

Figure 1. Angle Section Block

THE APPARATUS SKETCHED BELOW IS SUGGESTED IN ORDER TO FACILITATE HANDLING REPRODUCIBILITY IN TECHNIQUE.

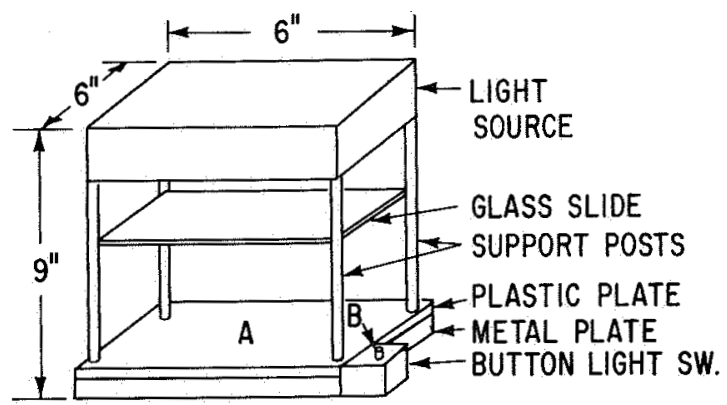

Figure 2. Staining Fixture

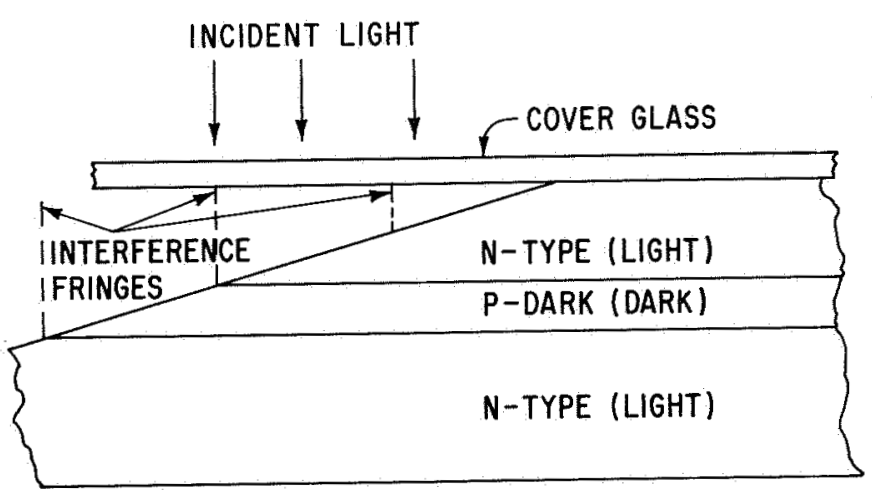

Figure 3. Measurement Setup 


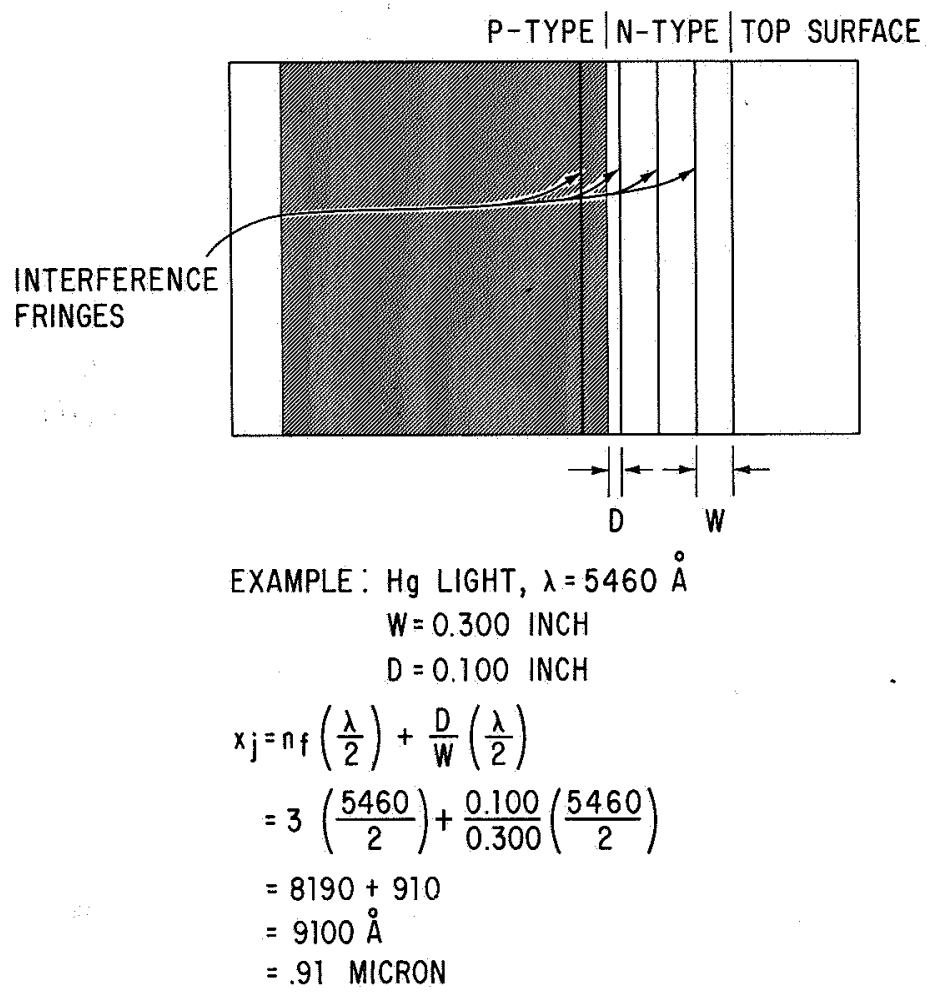

Figure 4. Junction Depth Measurement 
Junction Formation (Base Width)

(Monochromatic Interference)

1. Purpose The purpose of this test is to measure the distance between two successive $\mathrm{P}-\mathrm{N}$ junctions. It employs the interference of monochromatic light reflected between the beveled surface of the applicable structure and a thin, flat glass slide.

2. Apparatus

2. 1 Metallurgical Microscope

Metalstar Trinocular Body AO No. 2200T-4

American Optical Company

(or equivalent)

2. 2 Polaroid Camera AO No. 1052A

American Optical Company

(or equivalent)

2. 3 Monochromatic light source.

2.3.1 Mercury, 5461A, green filter.

LaPine Scientific Company, Mode1 216-01

(or equivalent)

\subsubsection{Sodium, $5893^{\circ} \mathrm{A}$ (optional)}

LaPine Scientific Company, Model 21 5-96

(or equivalent)

2. 4 High Intensity Light Fixture, Sun Gun II

Eastman Kodak

(or equivalent)

2. 5 High intensity bulb, $3400 \mathrm{~K}$ movie lamp, type DWY.

Sylvania Electric

(or equivalent)

2. 6 Hot plate, 6" $\times 6$ 6", electric

Temco, Type 1900

Corning, pyroceram, PC-35

(or equivalent) 
2. 7 Polyethylene beaker

(any source)

2.8 Glass plate, 6" 6 6"

(any source)

\section{Materials}

3. 1 Hydrofluoric acid, $49 \%$, Reagent grade.

3. 2 Nitric acid, concentrated, Reagent grade.

3. 3 Acetic acid, glacial, Reagent grade.

3. 4 Trichloroethylene, Electronic grade.

3. 5 Acetone, Electronic grade.

3. 6 Apiezon wax.

3. 7 Polishing compound, 1 micron, Linde "A".

3. 8 Cover glass, microscope $24 \times 30 \mathrm{~mm}$ No. 1-1/2 thinness. Corning, or equivalent.

4. Procedure

4. 1 Preparation

4. 1. 1 Place an angle section block, (Figure 1), on a hot plate adjusted to a temperature slightly above the melting point of apiezon wax $\left(150-160^{\circ} \mathrm{C}\right)$.

4. 1. 2 Place a small amount of wax in the sample niche.

4. 1.3 Insert the structure to be sectioned into the sample niche, pattern up.

NOTE: For ease of measurement, passivating layer should be removed.

4. 1.4 With tweezers or applicators, orient the junction over the edge of the bevel and seat the sample.

4. 1. 5 Remove the block from the hot plate and allow to cool. 
4. 1.6 Mix a slurry of Linde "A" and water.

4. 1.7 Place a quantity of the slurry on a flat glass plate.

4. 1.8 Invert the angle block and place the $5^{\circ}$ bevel against the glass plate.

4. 1.9 Adding Linde "A" as necessary, polish the specimen to a microscopically smooth finish.

4. 1. 10 Mix a junction stain of the following composition:

$$
1 \mathrm{HF}: 3 \mathrm{HNO}_{3}: 20 \mathrm{HA}_{\mathrm{C}}
$$

4. 1. 11 Rinse the mounted specimen thoroughly in deionized water, and blow dry with a nitrogen gun.

4. 1. 12 Place the cleaned specimen in the staining apparatus, Figure 3, or beneath a "Sun Gun".

4. 1. 13 Illuminate the specimen for fifteen seconds.

4.1.14 Prior to turning off the light, place the $1: 3: 20$ staining solution on the specimen, using a cotton-tipped applicator.

4. 1. 15 Allow the stain to remain on the sample for five to ten seconds.

4. 1.16 Turn off the light and quickly rinse in DI water.

4. 1.17 Examine the specimen for adequate junction delineation. Additional polishing or staining may be necessary.

4. 1.18 Return mounted specimen to the hot plate until the wax melts.

4. 1. 19 Clean the dismounted specimen with TCE and actene. Allow to dry. 


\section{2 Measurement}

4.2. 1 Place the specimen on the microscope stage with a microscope cover glass over the junction. See Figure 3 .

4.2. 2 Adjust magnification and focus until the junction (s) of interest and interference fringes are clear.

4.2.3 Starting with the top surface, count the number of fringes to the first junction, or just short of it if the junction lies between fringes $n^{\prime} f$.

4. 2. 4 If the junction is not on a whole fringe, measure the distance between fringes, w, with a precision rule.

4.2. 5 Measure the distance from the last fringe to the junction " $\mathrm{D}$ ".

4.2. 6 Repeat Steps 4.2 .3 and 4.2. 5 for the next junction.

4. 2. 7 Calculate the base width, $\Delta x$, from the following equation:

$$
\begin{aligned}
\Delta x & =\left[n f\left(\frac{\lambda}{2}\right)+\frac{D}{w}\left(\frac{\lambda}{2}\right)\right] \\
& -\left[n^{\prime} f\left(\frac{\lambda}{2}\right)+\frac{D}{w}\left(\frac{\lambda}{2}\right)\right]
\end{aligned}
$$

See Figure 4.

\section{Calibration}

Not applicable.

\section{Accuracy}

Accuracy obtainable is \pm 0.1 micron.

\section{Test Frequency}

This test should be performed on a sample from each run. 


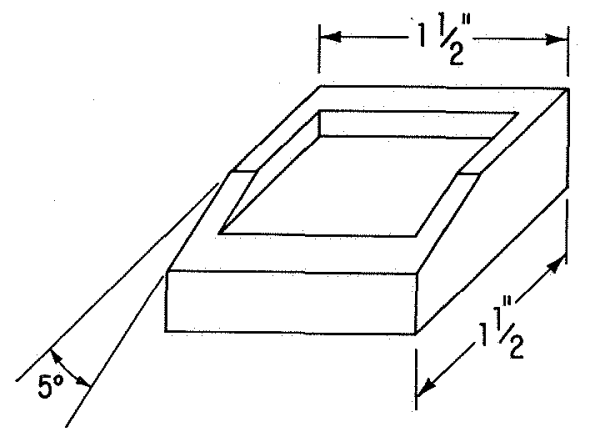

Figure 1. Angle Section Block

THE APPARATUS SKETCHED BELOW IS SUGGESTED IN ORDER TO FACILITATE HANDLING REPRODUCIBILITY IN TECHNIQUE.

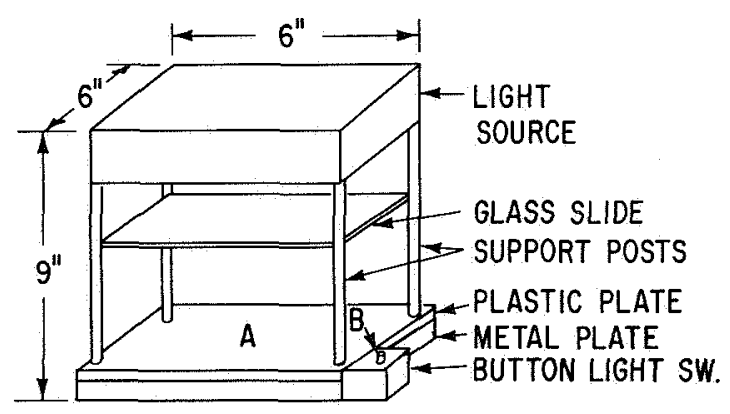

Figure 2. Staining Fixture

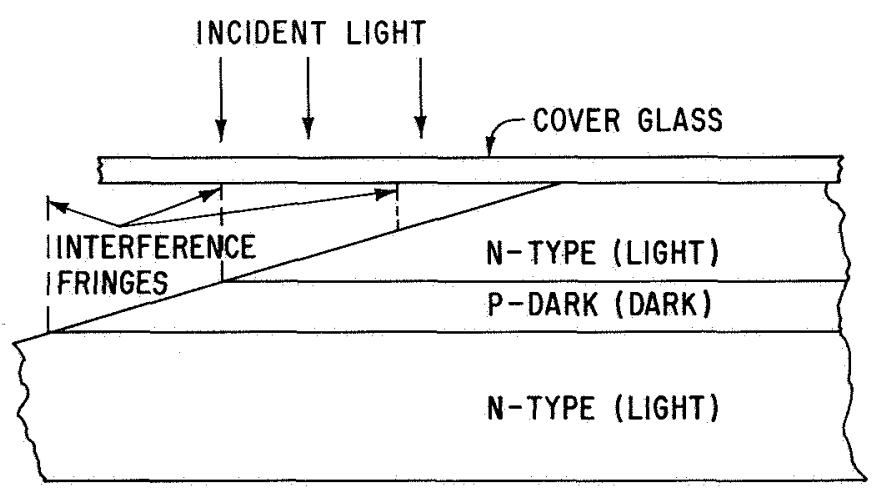

Figure 3. Measurement Setup 


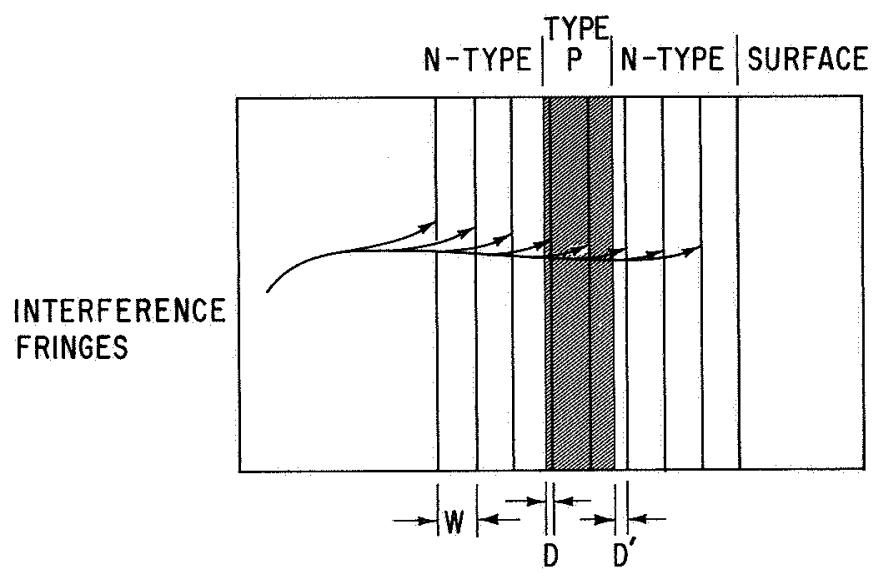

EXAMPLE: $\mathrm{Hg}$ LIGHT, $\lambda=5460 \mathrm{~A}$, GREEN FILTER

$W=0.300 \mathrm{INCH}$

$D=0.050 \quad \mathrm{INCH}$

$D=0.100 \mathrm{INCH}$

$\triangle \mathrm{X}=\mathrm{BASE}$ WIDTH

$$
\begin{aligned}
\Delta x & =\left[n_{f}\left(\frac{\lambda}{2}\right)+\frac{D}{W}\left(\frac{\lambda}{2}\right)\right]-\left[n_{f}^{\prime}\left(\frac{\lambda}{2}\right)+\frac{D^{\prime}}{W}\left(\frac{\lambda}{2}\right)\right] \\
& =\left[5\left(\frac{5460}{2}\right)+\frac{0.050}{0.300}\left(\frac{5460}{2}\right)\right]-\left[3\left(\frac{5460}{2}\right)+\frac{0.100}{0.300}\left(\frac{5460}{2}\right)\right. \\
& =[13650+455]-[8190+910] \\
& =14105-9100 \\
& =5005 \AA=.50 \text { MICRON }
\end{aligned}
$$

Figure 4. Junction Depth Measurement 


\author{
Method 135 \\ Junction Formation \\ (Sheet Resistance)
}

1. Purpose

The purpose of this test is to measure the sheet resistance of any structure, whether formed by an initial doping or the final diffusion. A lapped test chip of opposite type from the dopant is checked for voltage drop across a four-point probe utilizing a constant-current power supply.

2. Apparatus

2. 1 Four-point probe, 0.050-inch spacing. (any source)

2. 2 Power supply, constant current

$0.1,1.0$, and $10 \mathrm{~mA}$ ranges. (any source)

2.3 Voltmeter, digital

Fairchild 7100

(or equivalent)

3. Materials

3. 1 Hydrofluoric acid, 49 percent, Reagent grade.

3. 2 Silicon slices, $P$ and $N$ type, lapped.

4. Procedure

\title{
4. 1 Preparation
}

4. 1. 1 Process a lapped slice, of opposite type from the dopant concentration under evaluation, through the predeposition and/or diffusion operation to be measured.

NOTE: The test chip should have a surface area in excess of one-half square inch. 
4. 1. 2 Allow the test chip to cool to room temperature.

4. 1.3 Etch the chip until all doped oxide is removed, approximately ten seconds in concentrated hydrofluoric acid.

4. 1. 4 Rinse the chip in deionized water and blow dry with a nitrogen gun.

\section{2 Measurement}

4.2. 1 Place the test chip upon the stage of the four-point probe. See

NOTE: If the furnace operation utilizes an upright wafer position, then either side may be measured. Test chips processed through horizontal operations must be probed on the surface exposed to doping.

4.2.2 Lower the probe until it touches the sample lightly.

4.2.3 Record the voltmeter reading.

4. 2. 4 Reverse polarity and note that both values are approximately the same.

4. 2. 5 If readings differ significantly, increase probe pressure slightly until agreement is reached.

4. 2. 6 If the current source is $100 \mu \mathrm{A}, 1 \mathrm{~mA}, 10 \mathrm{~mA}$, etc., the voltmeter will read directly in sheet resistance with application of the proper decimal point. Example:

$$
\begin{aligned}
& 1=100 \mu \mathrm{A} \\
& V=50 \mu \mathrm{A} \\
& \frac{\mathrm{V}}{1}=\frac{50}{100}=0.5 \Omega / \square
\end{aligned}
$$

5. Calibration

\section{1 Probe Spacing}

5. 1. 1 Cover a $1^{\prime \prime} \times 3^{\prime \prime}$ glass microscope slide with aluminum foil. 
5. 1.2 Obtain an impression of the four probe points.

5. 1.3 Measure spacing with any optical comparator.

5. 1. 4 Adjust spacing per probe manufacturer's instruction.

6. Accuracy

Accuracy obtainable is approximately $\pm 10 \%$

\section{Test Frequency}

This test should be performed after each predeposition or diffusion. 
Method 135

\section{FOUR POINT PROBE - SET-UP}

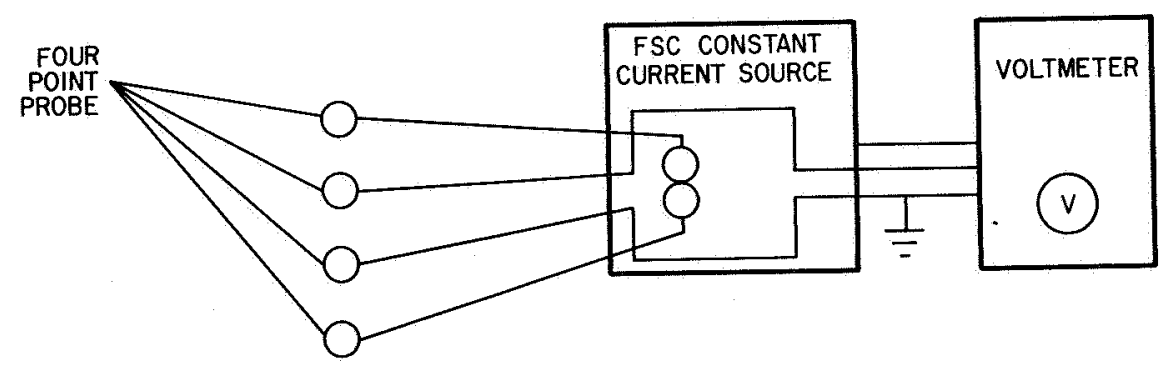

Figure 1. 


\author{
Method 136 \\ Junction Formation \\ (Furnace Temperature Profile)
}

1. Purpose This test is applicable to the temperature profiling of diffusion, oxidation or alloying furnaces. It utilizes a thermocouple and millivolt potentiometer.

2. Apparatus

2. 1 Millivolt Potentiometer

Leeds and Northup

Model No. 8662 or 8686

(or equivalent)

2. 2 Quartz Thermocouple Sheath

$6 \mathrm{~mm}$

(any source)

2. 3 Platinum-Rhodium Thermocouple

(any source)

3. Materials

None.

4. Procedure

4. 1 Position Potentiometer in front of furnace so that thermocouple will be able to be moved within the furnace tube as required.

4. 2 Insert thermocouple and thermocouple sheath into that furnace until the end of the sheath is flush with the end of the quartz tube.

4. 3 Connect thermocouple to potentiometer. Make sure that the red lead is connected to the terminal marked with the minus sign (-) and the other lead connected to the terminal marked with the plus sign $(+)$. 
4. 4 Check to see if all gases usually flowing through the furnace except the source, are flowing at their usual rates of flow.

4. 5 Allow thermocouple and potentiometer to stand for 10 minutes for temperature to stabilize.

4. 6 Calibrate thermocouple prior to use. Paragraph 5.

4. 7 Find temperature-millivolt equivalent of expected furnace temperature from temperature-millivolt equivalent chart and set the main scale of the potentiometer to read this value.

$$
\text { Example: } \quad 1000^{\circ} \mathrm{C}=10.471
$$

4. 8 Place the end of the thermocouple at the first location of interest and allow 30 seconds for the temperature to stabilize.

NOTE: Usually it is most convenient to commence at the point furthest from the furnace mouth.

4. 9 Tap the " $L$ " key and note the deflection on the galvanometer. Adjust the setting on the main scale until little or no deflection appears. Repeat using " $\mathrm{H}$ " key.

4. 10 Convert the final millivolt reading back to temperature by using the temperature-millivolt equivalent chart.

$$
\text { Example: } \quad 10.445 \text { millivolts }=998^{\circ} \mathrm{C}
$$

4. 11 Record the temperature for that point after making a correction to any the rmocouple error.

4. 12 Repeat 4.8 to 4.11 until profile is completed.

4. 13 At the completion of profiling disconnect thermocouple as follows:

\section{13. 1 Model 8662}

4. 13. 1. 1 Place metal bar across two terminals in upper corner.

4.13.1.2 Push lamp switch off.

4. 13.1.3 Disconnect the rmocouple leads. 2 
4.13.2 Model 8686

4.13.2.1 Turn function switch to "Galv. off" position.

4.13.2.2 Disconnect thermocouple leads

4. 13.2.3 Close lid of potentiometer.

4. 14 Slide thermocouple back into thermocouple sheath, remove both from furnace and place in storage tube fastened to the furnace provided for that specific thermocouple.

\section{Calibration}

5. 1 Thermocouple should be checked for error against a melting point standard.

\section{2 Potentiometer}

\subsection{Model 8662}

5. 2. 1. 1 Pull lamp switch up. Figure 1.

5.2. 1.2 Check to make sure that metal bar is across two terminals in upper corner of potentiometer.

5. 2. 1.3 Adjust "zero adjust" knob until galvanometer is balanced at zero.

5. 2. 1. 4 Loosen terminals holding metal bar and position bar so that it is away from one of the terminals. Figure 2 .

5. 2. 1. 5 Place "snap switch in "S. C. " position."

5.2.1.6 Tap the "Low" key and note deflection. Adjust "Coarse knob on side of case until there is little or no deflection on the galvanometer when the "Low" key is depressed." 
5. 2. 1.7 Tap the "High" key and note deflection. Adjust the fine knob on the side of the case until there is no deflection.

5. 2. 1.8 Reposition snap switch in "EMF" position.

5.2.1.9 Note temperature on thermometer taped to case. Figure 3.

5. 2. 1. 10 Find millivolt equivalent on temperaturemillivolt equivalent chart.

$$
\text { Example: } 27^{\circ} \mathrm{C}=0.154 \mathrm{MV}
$$

5.2.1.11 With the "Reference Junction" knob, adjust the "Reference Junction" scale to read the temperature-millivolt equivalent value in black.

\subsubsection{Mode1 8686}

5.2.2. 1 Turn function switch to "standarized" position. Figure 1.

5. 2.2. 2 Make sure both " $L$ " and " $\mathrm{H}$ " keys are in the up position.

5.2.2.3 Check zero reading. Adjust with the coarse and fine "zero adjust" knobs until the galvanometer is balanced at zero.

5. 2. 2. 4 Check if function switch is still in "standarized" position. Figure 2.

5. 2.2. 5 PUSH the "L" key and note deflection. Adjust "Battery Coarse" knob until there is little or no deflection, when " $L$ " key is depressed.

5.2.2.6 PUSH the "H" key and note deflection. Adjust "Battery Fine" knob until there is no further deflection when " $\mathrm{H}$ " key is depressed. 
5.2.2. 7 Note temperature on thermometer taped to case. Figure 3 .

5.2.2.8 Find millivolt equivalent on . temperature-millivolt equivalent chart.

5.2. 2.9 Turn function switch to "Ref. Jct." position.

5.2.2.10 Using knob "C", adjust the scale to read the temperature millivolt equivalent.

5.2.2.11 Tap the "L" key and note deflection on galvanometer. Adjust the "Reference Junction" knob until there is no further deflection when " $L$ " key is depressed.

5.2.2.12 Repeat using "H" key.

5.2.2.13 Turn the function switch to "T. C." measure.

\section{Accuracy}

Accuracy obtainable is $\pm 1^{\circ} \mathrm{C}$.

\section{Test Frequency}

This test should be performed at least once per working day. 


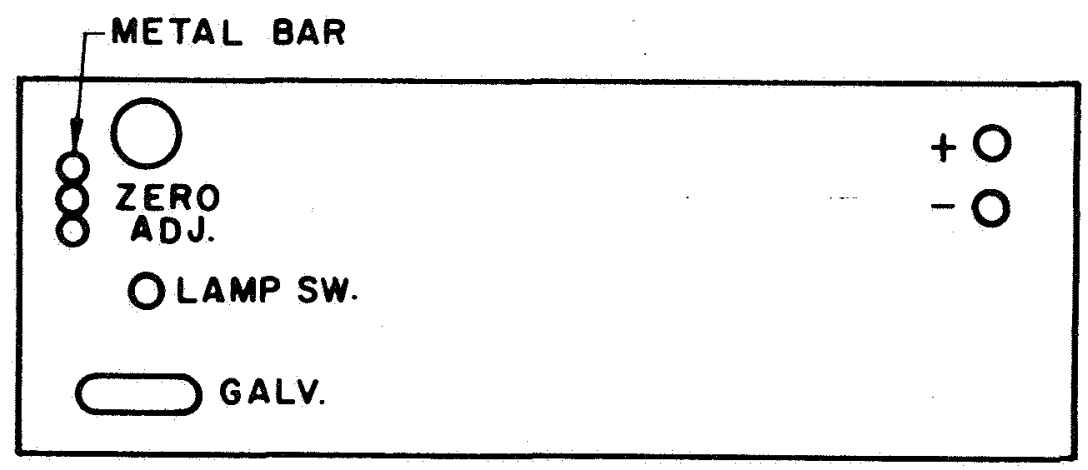

MODEL 8662

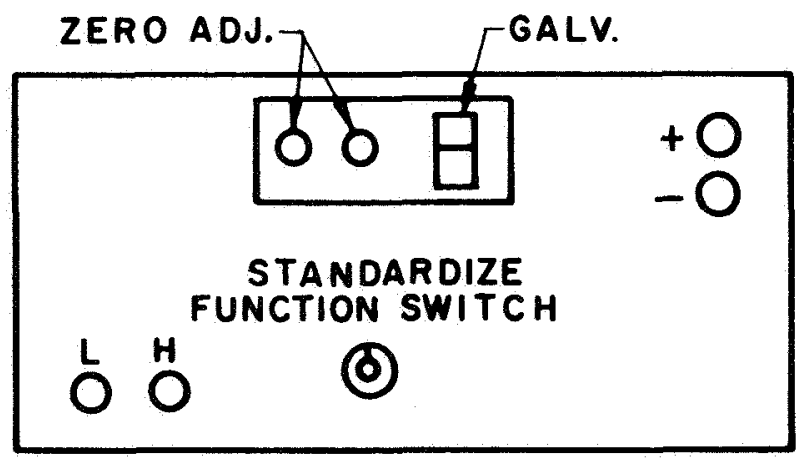

MODEL 8686

Figure 1 

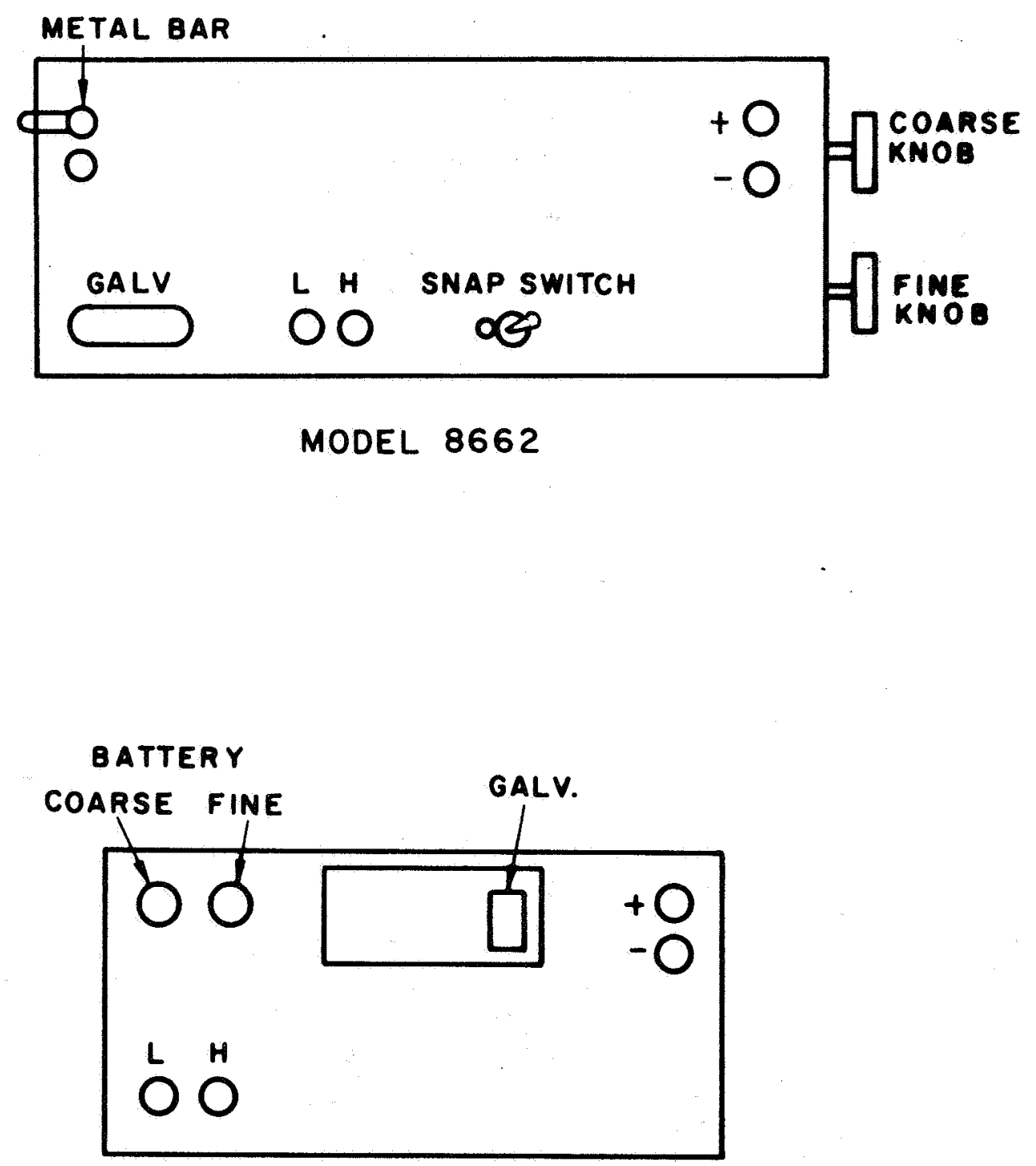

MODEL 8686

Figure 2 


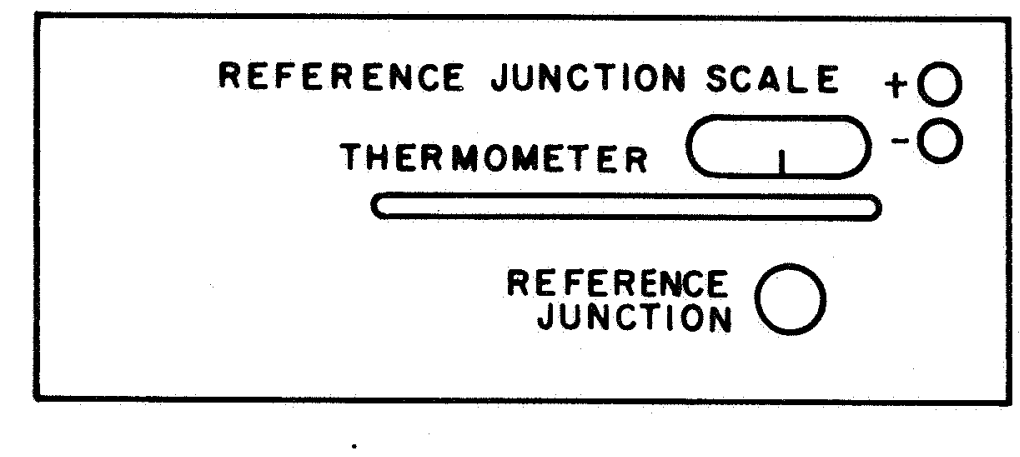

MODEL 8662

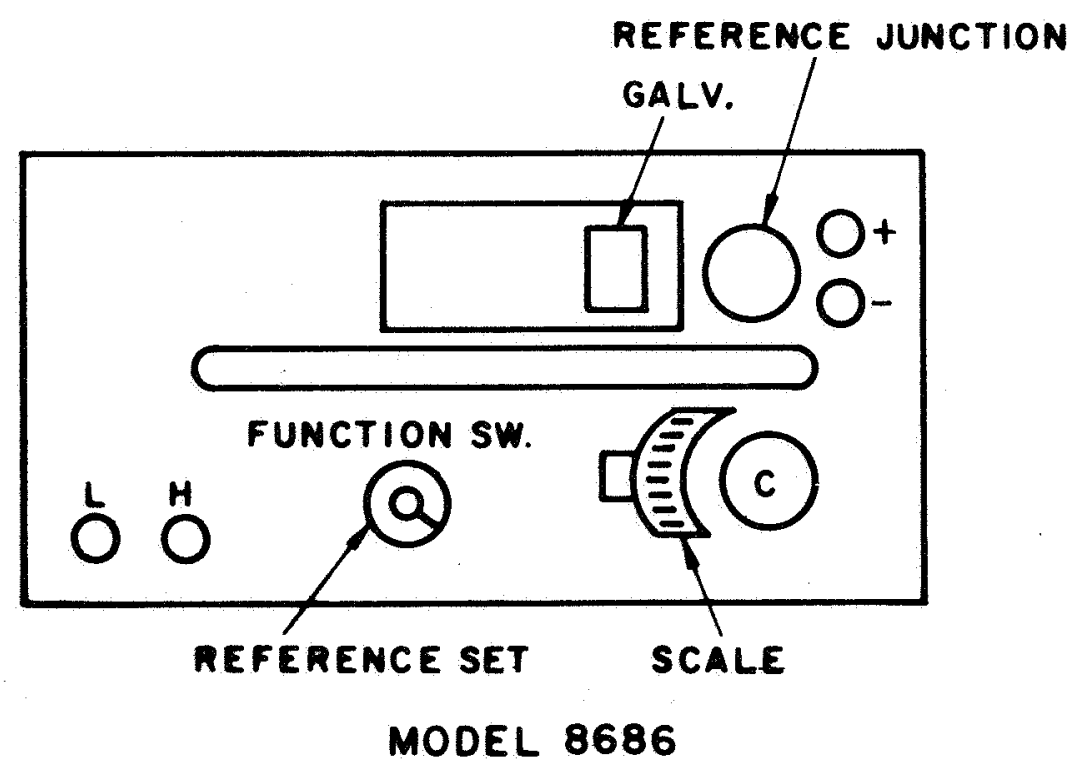

Figure 3 


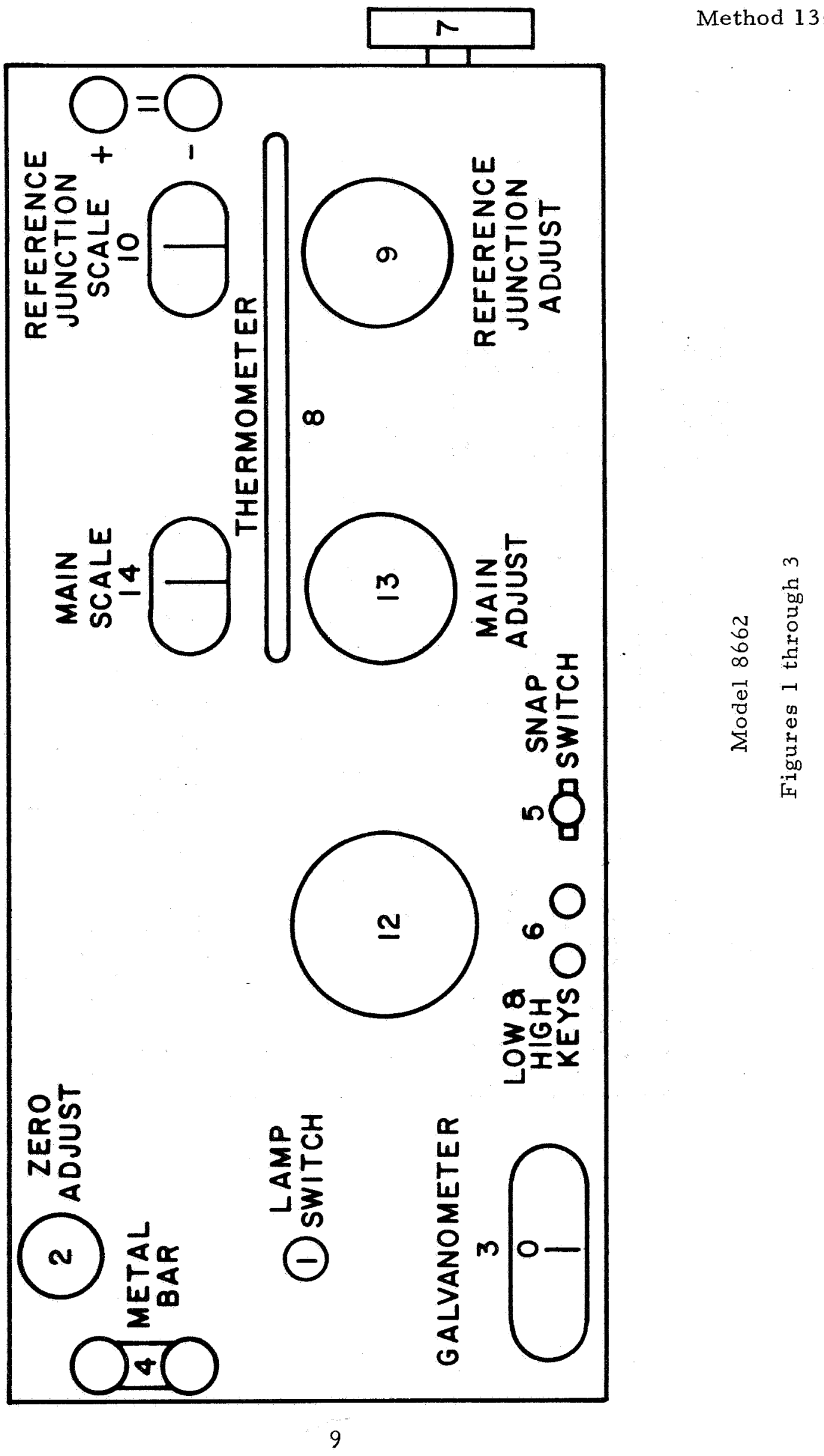


Method 136

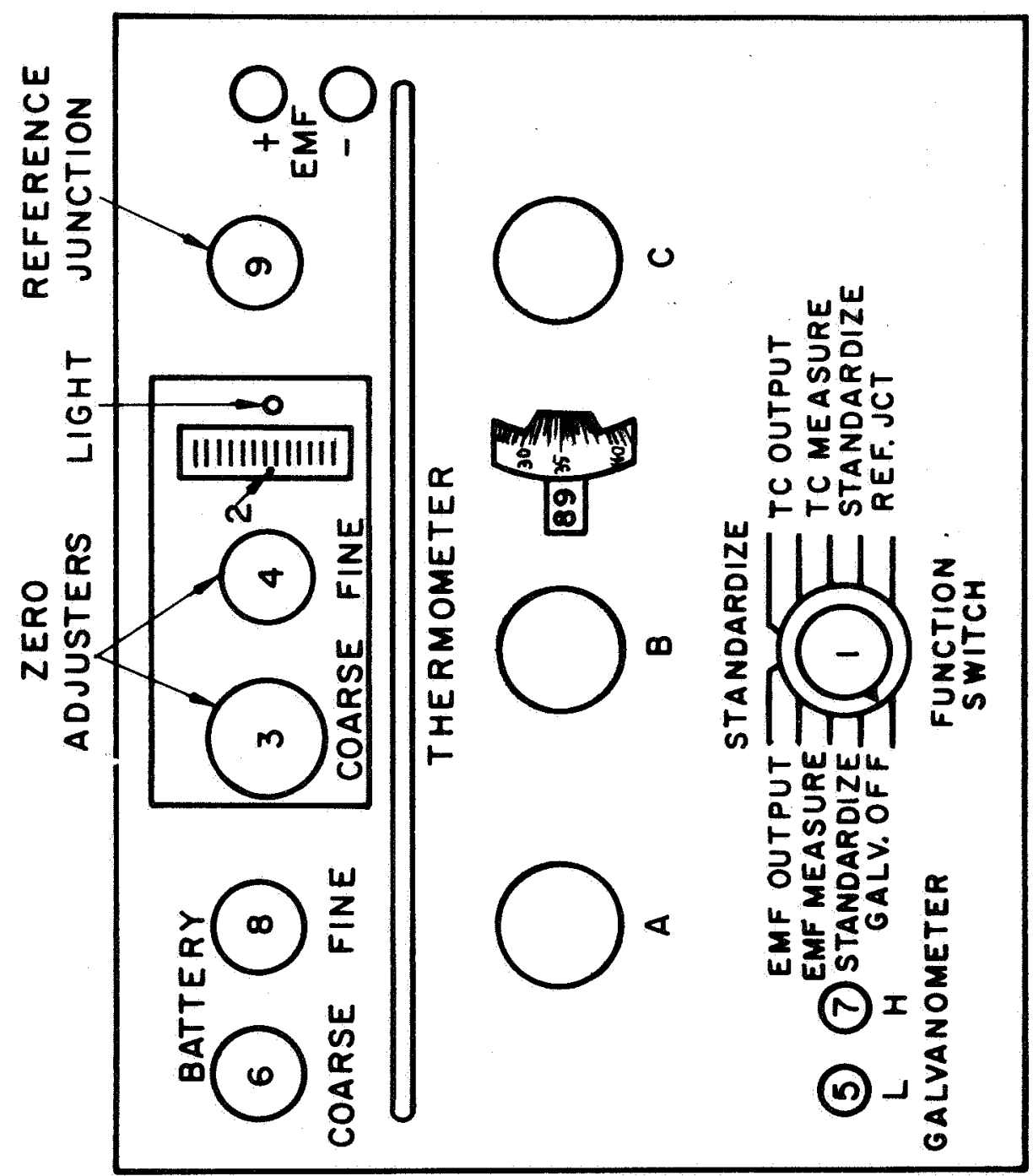

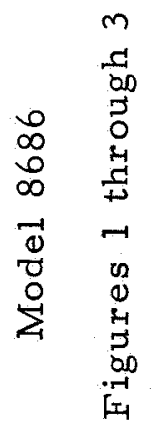




\author{
Method 137 \\ Junction Formation \\ (Flow Profile of Gases Around Slice Holder)
}

1. Purpose

This method may be used to evaluate the uniformity of gas flow about the slice holder, both. vertically and horizontally. It is applicable to those furnace operations where the slice is doped and utilizes uniform sheet resistance as the indicator for gas flow.

2. Apparatus

2. 1 Four-point probe, 0.050-inch spacing. (any source)

2. 2 Power supply, constant current

$0.1,1.0,10 \mathrm{~mA}$ ranges

(any source)

2. 3 Voltmeter, digital

Fairchild 7100

(or equivalent)

3. Materials

3. 1 Hydrofluoric acid, $40 \%$, Reagent grade.

3. 2 Silicon slices, $\mathrm{P}$ and $\mathrm{N}$ type, lapped.

4. Procedure

\title{
4. 1 Preparation
}

4.1. 1 Process lapped slices, of opposite type from the dopant concentration under evaluation, through the predeposition and/or diffusion operation to be measured.

NOTE: Samples should be placed uniformly about the slice holder and may have numbers scribed upon them for ease of identification. See Figure 1. 
4. 1.2 Allow the test wafers to cool to room temperature.

4. 1.3 Etch the wafers until all doped oxide is removed, approximately ten seconds in concentrated hydrofluoric acid.

4. 1. 4 Rinse the wafers in deionized water and blow dry with a nitrogen gun.

\section{2 Measurement}

4.2. 1 Place the test wafers upon the stage of the fourpoint probe. See Figure 2.

NOTE: If the furnace operation utilizes an upright wafer position, then either side may be measured. Test wafers processed through horizontal operations must be probed on the surface exposed to doping.

4.2. 2 Lower the probe until it touches the sample lightly.

4.2. 3 Record the voltmeter reading.

4.2. 4 Reverse polarity and note that both values are approximately the same.

4.2. 5 If readings differ significantly, increase probe pressure slightly until agreement is reached.

4.2. 6 If the current source is $100 \mu \mathrm{A}, 1 \mathrm{~mA}, 10 \mathrm{~mA}$, etc., the voltmeter will read directly in sheet resistance with application of the proper decimal point. Example:

$$
\begin{aligned}
1 & =100 \mu \mathrm{A} \\
V & =50 \mu \mathrm{V} \\
\frac{V}{1} & =\frac{50}{100}=0.5 \Omega / \square
\end{aligned}
$$

4.2. 7 Read $\frac{V}{I}$ in five locations on each slice as shown in Figure 3.

4.2. 8 Row, column and vertical average may be used to give a dopant pattern throughout the slice holder. 
5. Calibration

5. 1 Probe Spacing

5. 1. 1 Cover a $1^{\prime \prime} \times 3^{\prime \prime}$ glass microscope slide with aluminum foil.

5. 1.2 Obtain an impression of the four probe points.

5. 1.3 Measure spacing with any optical comparator.

5. 1. 4 Adjust spacing per probe manufacturer's instruction.

6. Accuracy

Accuracy obtainable is approximately $\pm 10 \%$.

7. Test Frequency

This test should be performed periodically for each predeposition furnace. 


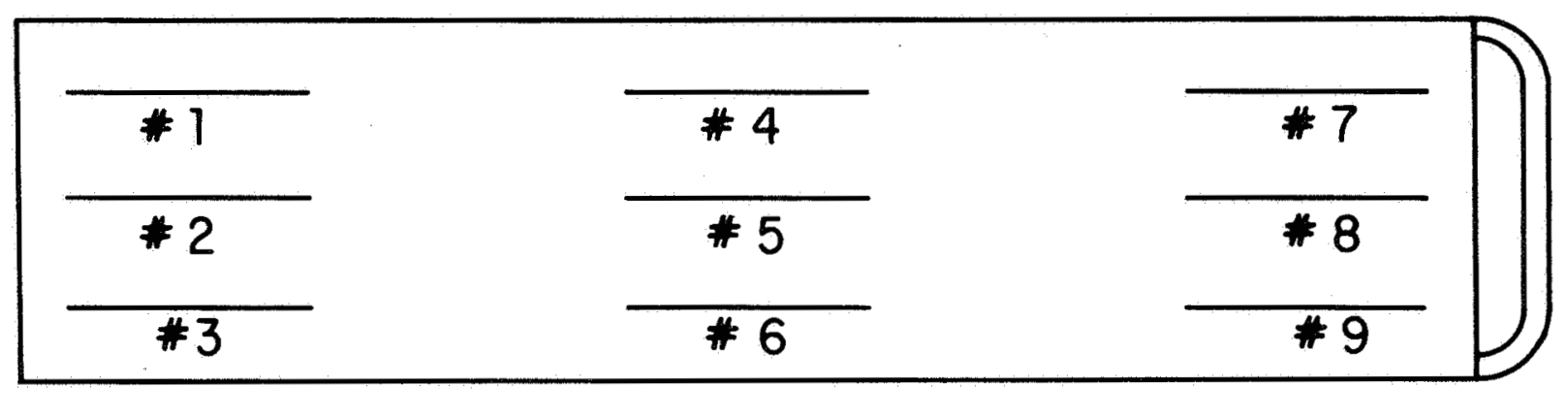

Figure 1. Sample Position in Boat 
FOUR POINT PROBE - SET-UP

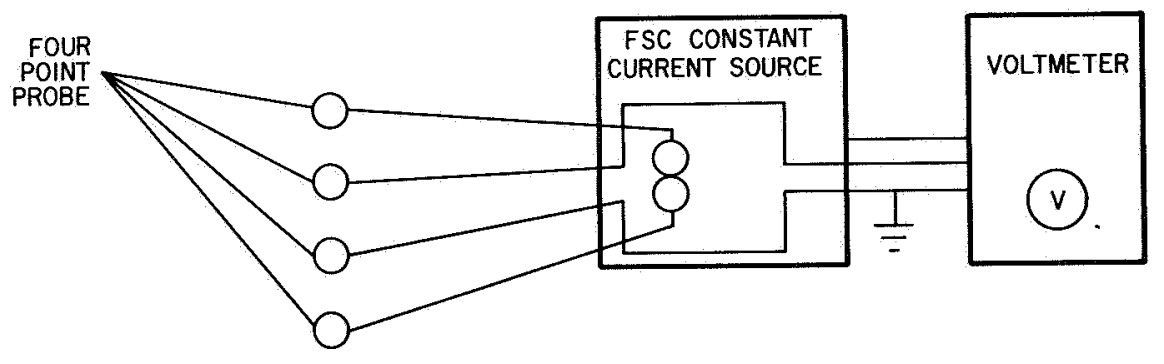

Figure 2. 
Method 137

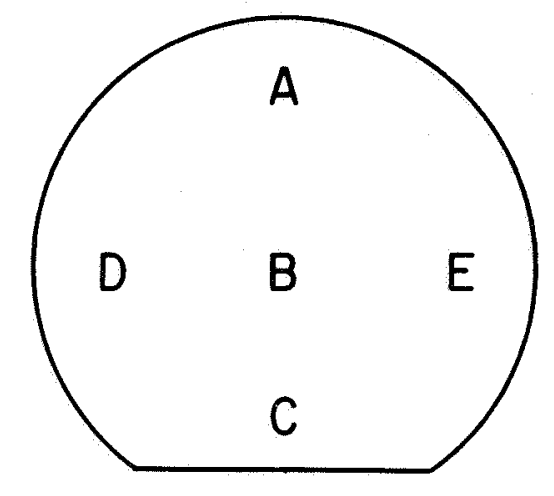

Figure 3. Sampling Pattern 
Method 138

Diode Isolation

(Breakdown Voltage)

1. Purpose The purpose of this method is to determine that the isolation technique will deliver an avalanche voltage greater than maximum working voltage.

2. Apparatus

2. 1 Two-point Prove, with micro-manipulator

(any source)

2. 2 Transistor Curve Tracer

Fairchild 6200

Tektronix 575

(or equivalent)

3. Materials

Not applicable:

4. Procedure

4. 1 Obtain integrated circuit wafer after base photomask etching.

4. 2 Place the wafer upon the probe stage. Figure 1.

4. 3 Select a circuit and probe the base cut-outs in any pair of adjoining isolation "islands".

4. 4 Read the isolation breakdown voltage from the voltmeter.

4. 5 Reverse the polarity of the power supply and note any change in voltage.

NOTE: This is particularly important where "buried collectors" are present and masking misalignment during isolation may cause premature breakdown. 
4. 6 Repeat 4.3 through 4.5 for the remaining isolation islands of the circuit selected in 4.3 , being careful to test all adjacent "pairs".

5. Calibration

Calibrate curve tracer per manufacturer's instructions.

6. Accuracy

Accuracy obtainable will be \pm one small grid division on the curve tracer.

7. Test Frequency

Each fabrication run should be sampled after isolation diffusion. 


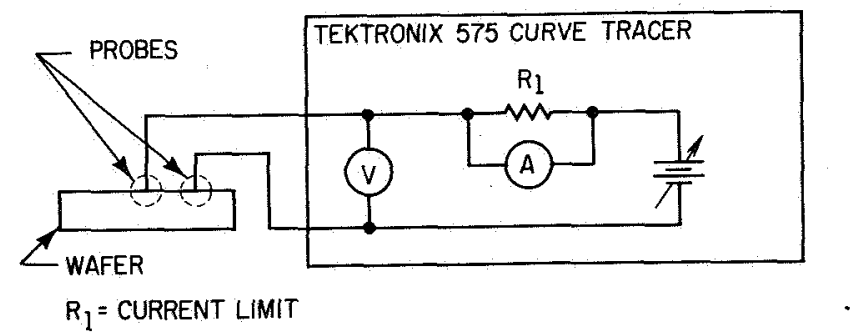

Figure 1. Diode Isolation (Breakdown Voltage) 
Method 139

Dielectric Isolation

(Breakdown Voltage)

1. Purpose The purpose of this method is to determine the voltage at which electrical breakdown of a speciman of electrical insulating material between two electrodes occurs under prescribed conditions of test.

2. Apparatus

2. 1 Ball Point Probes and Curve Tracer

Tektronix 575

(or equivalent)

2. 2 Transformers

2KVA

(any source)

2. 3 Circuit-Interrupting Device

(any source)

2. 4 Motor Driven Autotransformer (AC)

(any source)

2. 5 Insulated High Voltage Probes

Anodicator Mode1 50

R. O. Hull Co.

(or equivalent)

\section{Materials}

Not applicable. 
4. Procedure

4. 1 Prepare wafer with metallized contact on back surface and metal pads on isolation islands for probing. Wafer may be probed with "ball probes" without metal pads. Figure 1.

4. 2 Definitions:

4.2.1 Dielectric Breakdown Voltage The voltage at which electrical breakdown of a specimen of electrical insulating material between two electrodes occurs under prescribed conditions of test.

4.2.2 Dielectric Strength The ratio of dielectric breakdown voltage to the thickness of an insulating material.

4.2.3 Flashover An electric discharge around the edge or over the surface of electrical insulation.

4.3 Method - Short Time Test - The voltage shall be increased from zero to breakdown at a uniform rate. The rate of rise sha11 be 100,500 , 1000 or $3000 \mathrm{~V} / \mathrm{sec}$, depending on the total test time required and the voltage-time characteristic of material. For the rate applicable to a given material, reference shall be made to the test method for that material.

4. 4 Slow-Rate-of-Rise Test - An initial voltage shall be applied, approximately equal to $50 \%$ of the breakdown voltage in the short-time test, or as specified in the material specification. The voltage shall then be increased at a uniform rate, as stated in the material specifications, up to the point of breakdown. The specified rate should be such as to give approximately the same voltage-time exposure of the test specimen as provided in the step-by-step test.

4. 5 Rate-of-Rise - The rate-of-rise of voltage shall not, for the short-time test, vary more than $\pm 20 \%$ from the specified rate at any point. It may be calculated from measurements of time required to raise the voltage between two selected values. Voltage control shall be obtained as indicated in voltage control equipment. 
4.6 Step-by Step Test - Unless otherwise specified, an initial voltage equal to $50 \%$ of the breakdown voltage $\pm 5 \%$, as obtained on the short time test, shall be applied to the test specimen. The voltage shall then be increased in equal increments and held for periods of time specified in the various material specifications. The change from each step to the next higher shall be made as rapidly as possible and the time of change included in the succeeding test interval.

NOTE: The means used to obtain the various voltage steps shall not introduce voltage transiants that increase the peak value more than $10 \%$.

\section{Calibration}

Calibration of equipment as per manufacturer's specifications.

\section{Accuracy}

As obtained from manufacturer's specifications on monitoring equipment.

7. Test Frequency

Each fabrication run should be sampled after isolation process. 
DIELETRIC ISOLATION (Breakdown Voltage) (D.C. METHOD)
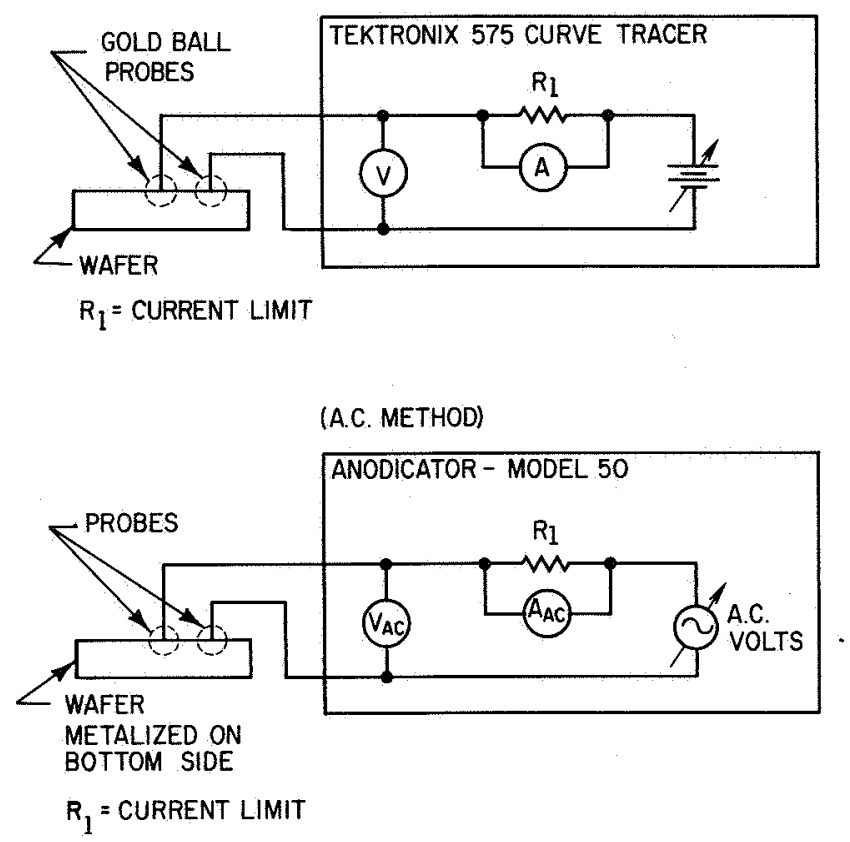

Figure. 1. 
Method 140

Isolation Leakage

1. Purpose The purpose of this method is to determine a means of evaluating leakage current from one Isolation Island to another.

2. Apparatus

2. 1 Two-point probe, with micro-munipulator

(any source)

2. 2 Leakage Tester

Wiltron, Model 205

(or equivalent)

2. 3 Picoammeter

Keithly, Model 416A

(or equivalent)

3. Materials

Not applicable.

4. Procedure

4. 1. Obtain integrated circuit wafer after base photomask etching.

4. 2 Place the wafer upon the probe stage. Included in Figure 1.

4. 3 Select a circuit, and probe the base cut-outs in any pair of adjoining isolation "Islands".

4. 4 Read the isolation leakage current on the picoammeter.

4. 5 Reverse the polarity of the power supply and note any change in current.

NOTE: This is particularly important whe re "buried collectors" are present. Masking misalignment during isolation may cause premature breakdowns, thus increase in current. 
4. 6 Repeat Steps 4.3 through 4.5 for the remaining isolation islands of the circuit selected in 4.3. Test isolations in adjacent "pairs".

5. Calibration

Calibrate equipment as per manufacturer's specifications.

6. Accuracy

Accuracy will be manufacturer's specification on monitoring equipment.

7. Test Frequency

Each fabrication run should be sampled after isolation diffusion. 
ISOLATION LEAKAGE

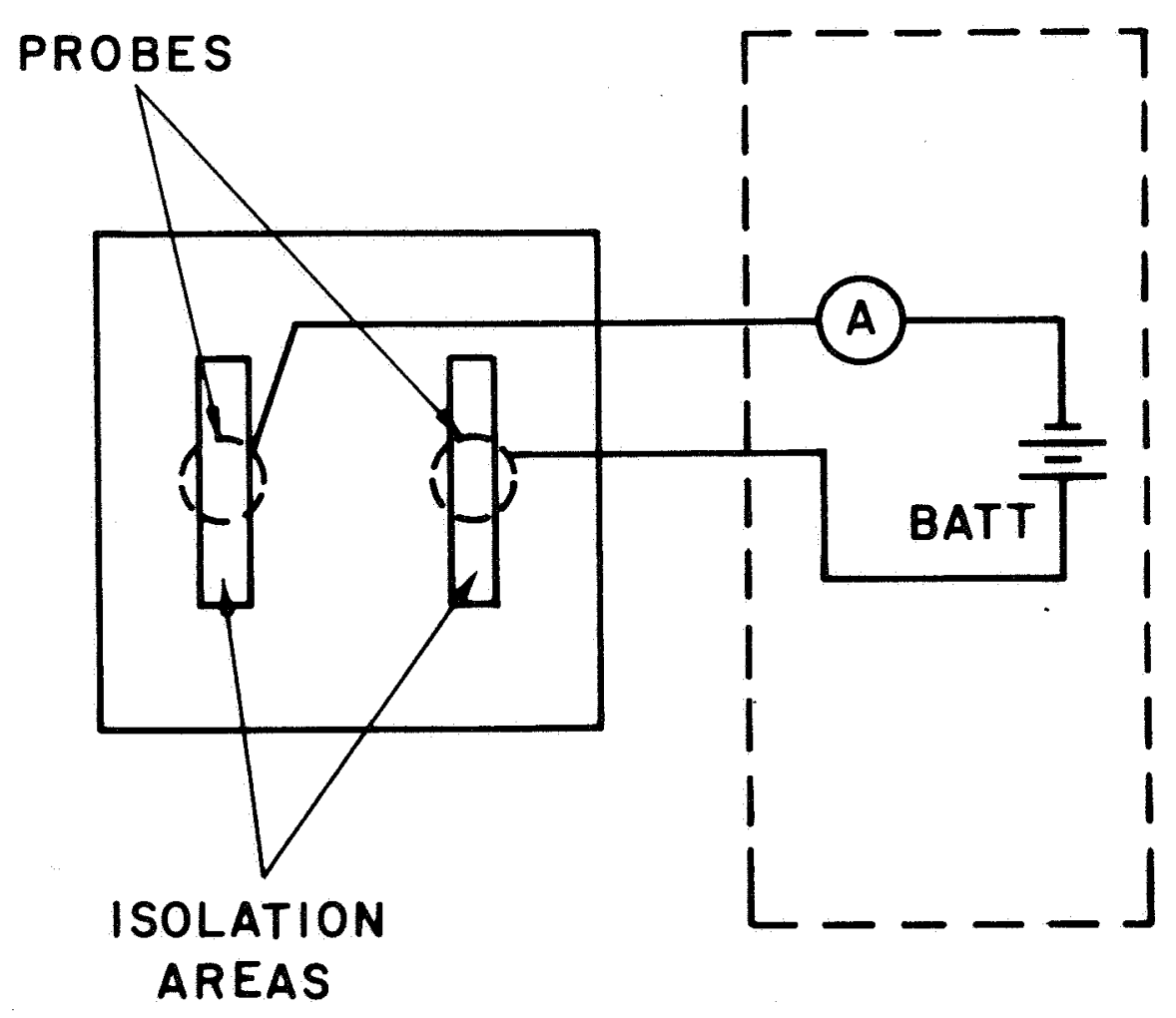

NOTES :

I. AMMETER KEITHLEY MODEL 416-A.

2. $V_{C C}$ SOURCE; ANY GOOD COMMERCIAL BATTERY.

3. COMPLETE SYSTEM SHOULD BE SHIELDED.

Figure 1. Isolation Leakage Setup 
Method 141

Thickness of Metallization

(Tolansky Interferometer)

1. Purpose This test procedure is applicable to the measurement of any thin film. It utilizes the interference of monochromatic light introduced into the optical system through a $95 \%$ mirror and reflected between that and the metallized specimen. The two mirrors are inclined to one another, causing the interference fringes to be normal to the edge of the measured layer. The horizontal displacement of any fringe enables one to calculate the layer thickness by the following relationship:

$$
\begin{aligned}
\frac{\mathrm{x}_{\mathrm{O}}}{\mathrm{D}} & =\frac{\frac{\lambda}{2}}{\mathrm{w}} \\
\text { Where } \mathrm{x}_{\mathrm{o}}= & \text { Film thickness } \\
\lambda= & \text { Wavelength of incident light } \\
\mathrm{w}= & \text { Distance between fringes } \\
\mathrm{D}= & \text { Distance any fringe is displaced. } \\
& \text { See Figures } 1 \text { and } 2 .
\end{aligned}
$$

2. Apparatus

2. 1 Metallurgical microscope

Metalstar trinocular body AO No. 2200T-4

American Optical Co.

(or equivalent)

2. 2 Polaroid camera AO No. 1052A

American Optical Co.

(or equivalent) 
2. 3 Interferometer jig

Fairchild design

See Figure 3.

2. 4 Monochromatic light source

2.4.1 Mercury, $5461 \AA$

LaPine Scientific Co. Model 216-01

( or equivalent)

2. 4. 2 Sodium, $5893 \AA$ (optional)

LaPine Scientific Co. Model 215-96

(or equivalent)

2. 5 Hot plate, 6" $\times 6$ ", electric

Temco, type 1900

Corning, Pyroceram PC-35

(or equivalent)

2. 6 Polyethylene beaker

(any source)

3. Materials

3. 1 Hydrofluoric acid, $49 \%$, reagent grade

3. 2 Acetic acid, glacial, reagent grade

3. 3 Trichloroethylene, electronic grade

3. 4 Acetone, electronic grade

3. 5 Apiezon wax

3. 6 Slides, glass microscope, $1 " \times 3 "$

4. Procedure

4. 1 Preparation

4. 1. 1 Process a polished test chip through the metallization operation to be evaluated.

NOTE: Form a step in the film either by etching or "shadowing" with a glass slide during evaporation. 
4. 1.2 Evaporate a $1000 \AA$ silver or aluminum film over the film to be examined.

NOTE: While silver has a higher index of reflectivity, it must not be allowed to tarnish prior to measurement.

\section{2 Measurement}

4.2.1 Place the specimen on the platform, mirrored side up.

4.2.2 Rotate the specimen on the platform until the step to be measured is normal to the front edge of the Fizeau plate.

4.2.3 Raise the platform until the specimen is in contact with the Fizeau plate by turning the knurled brass elevation nut. This will give maximum fringe spacing.

4.2. 4 If a smaller fringe spacing is desired, the platform may be raised slightly, reducing the wedge angle, $\theta$, and, as a result, fringe spacing.

4. 2. 5 Make a photomicrograph.

4.2. 6 Measure distance between fringes on the photomicrograph, with a precision rule. (w)

4. 2.7 Measure distance which any fringe is displaced as it passes across the film step. (D)

4.2.8 Calculate film thickness, $x_{0}$, using the relationship

$$
\frac{x_{0}}{D}=\frac{\frac{\lambda}{2}}{w}
$$

\section{Calibration}

Not applicable. 
6. Accuracy

Accuracy obtainable increases with increasing film thickness.

6. 1 For films greater than 0.2 microns, $\pm 10 \%$.

6.2 For films less than 0.2 microns, $\pm 20 \%$.

7. Test Frequency

This test should be performed on a periodic basis. 


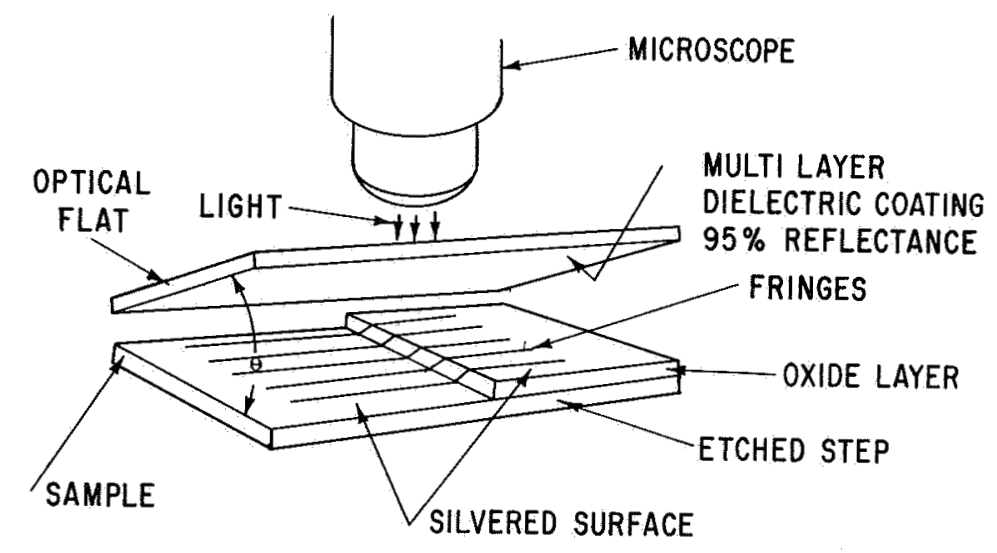

Figure 1. Tolansky Interferometer Setup 


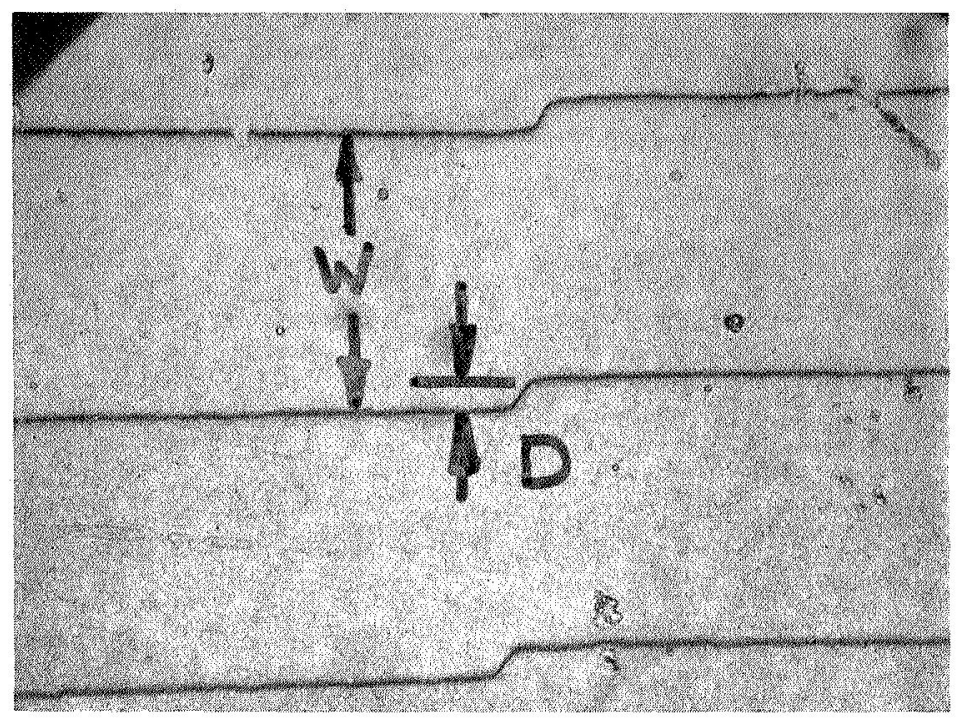

Figure 2. Film Measurement

Sodium, $\lambda=5890 \AA$

$$
\begin{aligned}
\mathrm{W} & =1.12 \text { inches } \\
\mathrm{D} & =0.14 \text { inches } \\
\mathrm{X}_{0} & =\mathrm{D} \frac{\frac{\lambda}{2}}{\mathrm{~W}} \\
& =0.14 \frac{\frac{5890}{2}}{1.12} \\
& =\frac{1.4 \times 2.945 \times 10^{2}}{1.12} \\
& =3.4 \times 10^{2} \\
& =340 \AA
\end{aligned}
$$

NOTE: A fixed error of $30-50 \AA$ becomes significant at this thickness range. 


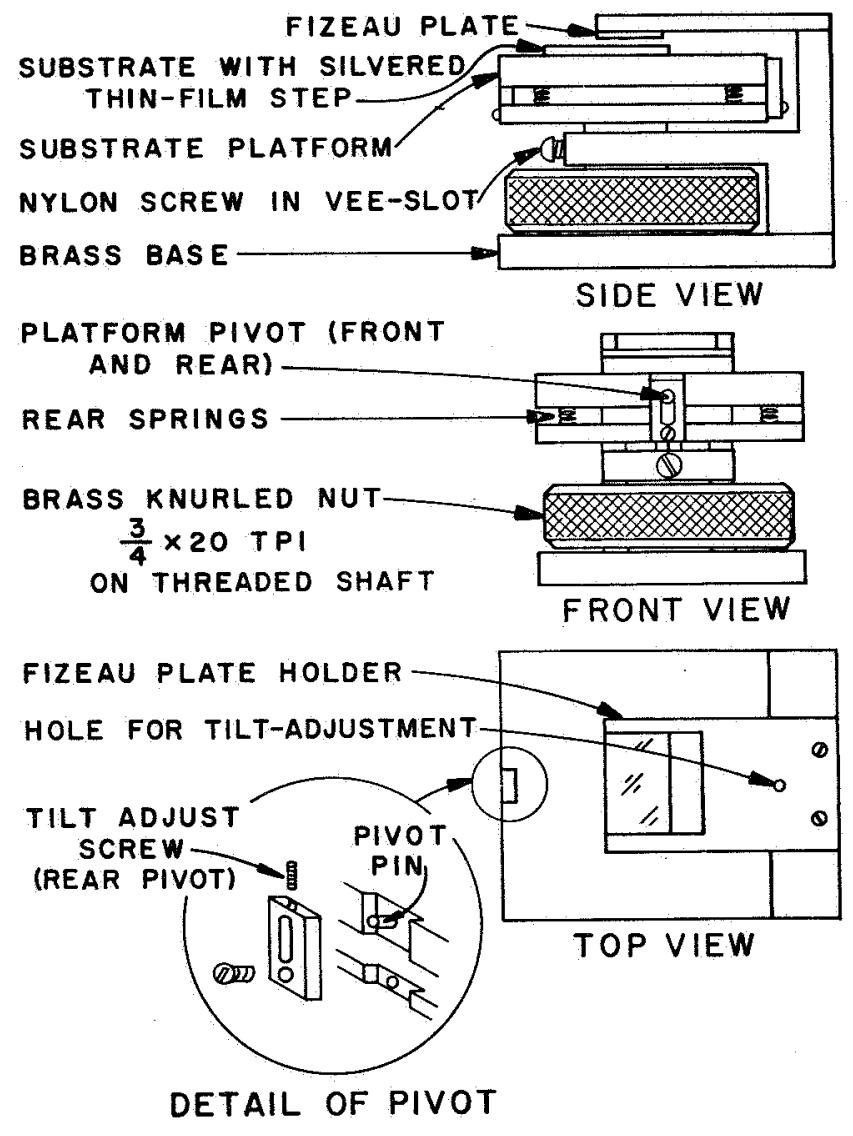

Figure 3. Interferometer Jig 


\section{Method 142}

Thickness of Metallization

(Sheet Resistance)

1. Purpose This procedure may be used for the indirect determination of a single metal evaporated film. It utilizes the measurement of a sheet resistance from which the film thickness is calculated using the following equation:

$$
w=\rho \frac{1 \mathrm{n} 2}{\pi \frac{V}{I}}(\text { For } w \rightarrow \text { Zero })
$$

$$
\text { Where: } \begin{aligned}
\mathrm{w} & =\text { Film thickness in } \mathrm{cm} . \\
\rho & =\text { Bulk resistivity of metal in } \Omega \mathrm{cm} . \\
\frac{V}{\mathrm{I}} & =\text { Sheet resistance. }
\end{aligned}
$$

\section{Apparatus}

2. 1 Four-point probe, 0.050-inch spacing. (any source)

2. 2 Power supply, constant current, $0.1,1.0,10 \mathrm{~mA}$ ranges.

(any source)

2. 3 Voltmeter, Digital

Fairchild 7100

(or equivalent) 
3. Materials

3. 1 Glass slides, $1^{\prime \prime} \times 3^{\prime \prime}$

4. $\quad$ Procedure

4. 1 Coat glass slides according to the process under evaluation.

4. 2 Place the slide to be measured upon the probe stage. See Figure 1.

4. 3 Lower the probe until it touches the sample lightly.

4. 4 Record the voltmeter reading.

4. 5 If the current source is $100 \mu \mathrm{A}, 1 \mathrm{~mA}, 10 \mathrm{~mA}$, etc., the voltmeter will read directly in sheet resistance with application of the proper decimal point.

4. 6 Using the formula from Para. 1, calculate the film thickness.

Example: $\quad \frac{\mathrm{V}}{\mathrm{I}}=0.006 \Omega$

$$
\begin{aligned}
& \text { Film - Aluminum } \\
& \rho=2.72 \times 10^{-6} \Omega-\mathrm{cm}, 25^{\circ} \text {, Aluminum } \\
& \mathrm{w}=\rho \frac{\ln 2}{\pi \frac{\mathrm{V}}{\mathrm{I}}} \\
& =\frac{2.72 \times 10^{-6} \times .693}{\pi \times .006} \\
& =\frac{2.72 \times 10^{-6} \times 6.93 \times 10^{-1}}{\pi \times 6 \times 10^{-3}} \\
& =\frac{2.72 \times 6.93 \times 10^{-4}}{\pi \times 6} \\
& =1 \times 10^{-4} \mathrm{~cm} \\
& =1 \text { micron } \\
& \mathrm{w}=10^{4} \AA
\end{aligned}
$$


5. Calibration

5. 1 Probe spacing

5. 1. 1 Cover a $1^{\prime \prime} \times 3^{\prime \prime}$ glass microscope slide with aluminum foil.

5. 1.2 Obtain an impression of the four probe points.

5. 1.3 Measure spacing with any optical comparator.

5. 1. 4 Adjust spacing per probe manufacturer's instructions.

6. Accuracy

Accuracy obtainable is approximately $\pm 10 \%$.

7. Test Frequency

This test should be performed on a sample slide from every evaporation. 
Method 142

FOUR POINT PROBE - SET-UP

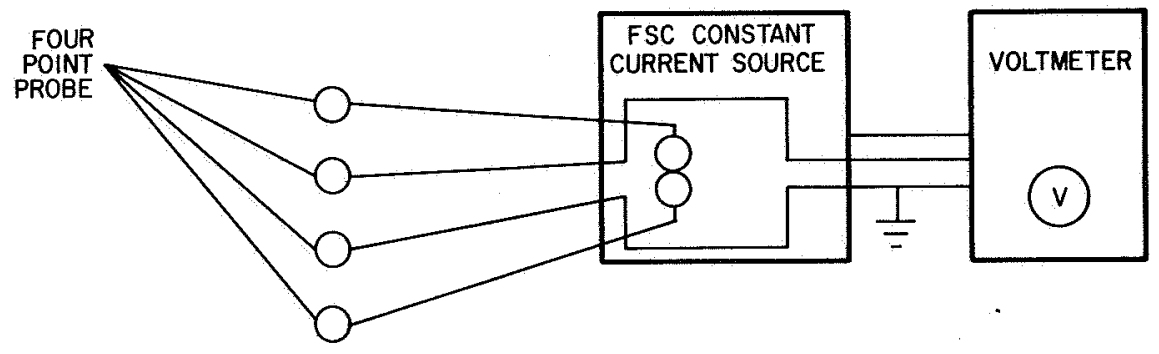

Figure 1. 


\section{Method 143 \\ Metallization Adhesion \\ (Pressure-Sensitive Tape)}

1. Purpose This method may be used to evaluate adhesion of the metallization film to the substrate. It utilizes a pressure-sensitive tape to remove metal with poor adhesion followed by a microscopic examination.

2. Apparatus

2. 1 Metallurgical microscope

Bausch and Lomb dynoptic No. 31-20-68-38

(or equivalent)

2. 2 Hot plate, 6" $\times 6^{\prime \prime}$, electric

Corning, Pyroceram PC-35

(or equivalent)

3. Materials

3. 1 Apiezon wax

3. 2 Slides, glass microscope, $1^{\prime \prime} \times 3 "$

3. 3 Pressure-sensitive tape, Scotch, 3/4", No. 810

4. Procedure

4. 1 Preparation

4. 1. 1 Process a polished, passivated substrate or a clean glass slide through the metallization operation to be evaluated.

Note: For a slide, omit 4.1.2 through 4.1.5

4. 1. 2 Place a glass slide on a hot plate set a temperature slightly higher than the melting point of apiezon wax $\left(150-160^{\circ} \mathrm{C}\right)$.

4. 1.3 Add a small quantity of wax to the slide. 
4. 1. 4 Place the sample upon the slide, metallization up, and seat it in the wax.

4. 1. 5 Remove the slide from the hot plate and allow it to cool.

4. 1.6 Apply one end of a four (4) inch length of pressuresensitive tape to the metallized surface. Insure contact across the maximum sample dimension with no voids or bubbles.

4. 1. 7 Hold the slide firmly against a solid surface and grasp the free end of the tape.

4. 1.8 Apply a force normal to the substrate surface and smoothly strip the tape from the metallization.

4. 1.9 The tape may be applied to a clean slide for preservation.

\section{2 Inspection}

4.2. 1 Place the sample on the stage of a metallurgical microscope.

4. 2. 2 At $100 x$, scan the metallized surface for any voids or missing metal.

\section{Calibration}

Not applicable.

\section{Accuracy}

This method should detect areas where metal is removed which are approximately 0.0005 square inches.

\section{Test Frequency}

7. 1 Evaluation of glass slides should be done for each evaporation.

7. 2 Substrate testing should be on a periodic basis. 
Method 144

Stability of Metallization

1. Purpose This method may be used to evaluate the stability of circuit metallization. It utilizes a high temperature storage test to detect formation of intermetallic compounds and a high temperature D-C bias test to observe "Electro-Migration". The test device is manufactured as process control devices are fabricated on a standard integrated circuit wafer.

\section{Apparatus}

2. 1 High Temperature Storage Oven $300^{\circ} \mathrm{C}$ air ambient

(any source)

2. 2 High Temperature D-C Bias Chamber $200^{\circ} \mathrm{C}, I_{F}=100 \mathrm{~mA}$ per device (any source)

2. 3 Curve Tracer Fairchild 6200 (or equivalent)

3. Materials

None 
4. Procedure

4. 1 Test Device Manufacture

4. 1. 1 Fabricate one or more test devices on each standard production wafer. See Figure 1.

4. 1.2 After dicing, separate test devices from production.

4. 1.3 Process through standard device as sembly.

4. 1. 4 Electrically screen test devices for continuity.

4. 2 Intermetallic Formation (Gold-Aluminum)

4.2. 1 Store this group of test devices at $300^{\circ} \mathrm{C}$ for approximately 200 hours.

4.2. 2 Cool to room temperature.

4.2.3 Monitor conductance at $10-20 \mathrm{~mA}$. Full scale. Any slope greater than $20 \Omega$ will indicate intermetallic formation.

\section{3 Electro-Migration}

4. 3. 1 Place devices in chamber at $200^{\circ} \mathrm{C}$ under $\mathrm{D}-\mathrm{C}$ bias which forces a current density of about $10^{6}$ amperes $/ \mathrm{cm}^{2}$ through each sample.

4.3.2 Store for approximately 200 hours.

4.3.3 Test for continuity at $10 \mathrm{~mA}$. Opens will be failures.

5. Accuracy

Not applicable. Current density for 4.3 should be set to deliver $10-20 \%$ open circuits.

\section{$6 \quad$ Calibration}

Not applicable. 


\section{Test Frequency}

This test should be performed periodically. 

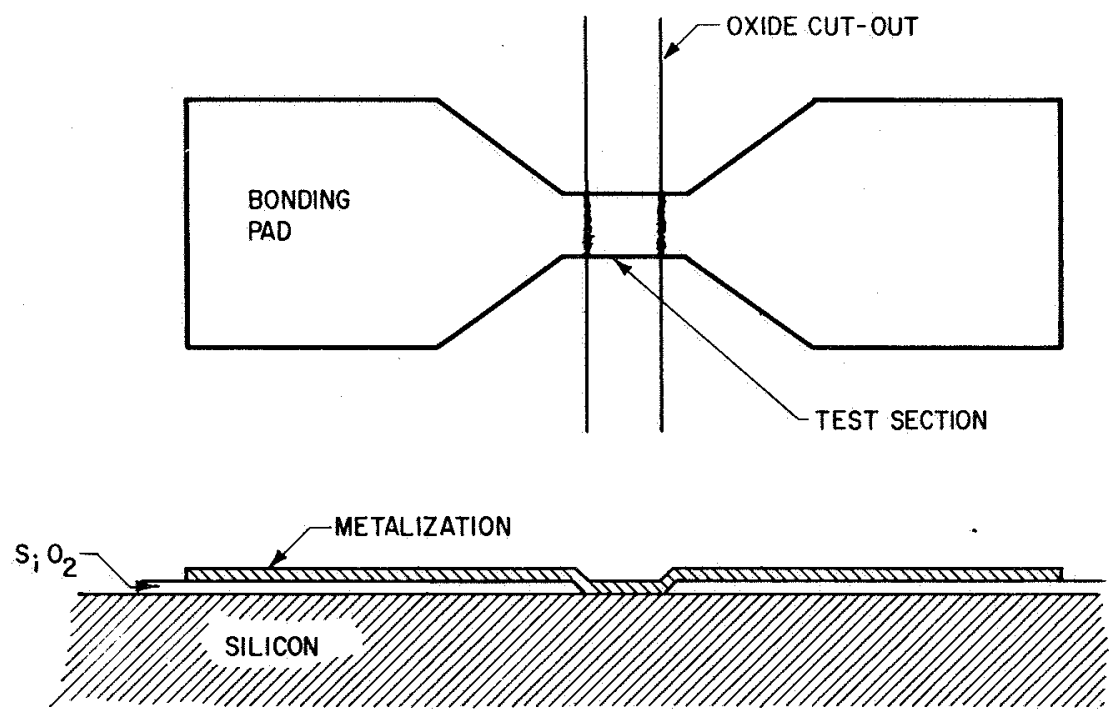

For Current Density 10 $10^{6}$ Amperes $/ \mathrm{cm}^{2}$

Metol Thickness $=10,000 \AA$

Test Section Width $=0.0004$ inch

Current (D.C) $=100 \mathrm{~mA}$

Figure 1. Metallization Test Device 
Method 145

Specific Resistivity of Metallization

1. Purpose This method may be used to indirectly measure the specific resistivity of a given evaporated metal film. It utilizes the comparison of measured and calculated thicknesses of the film.

$$
\begin{aligned}
\rho_{\mathrm{s}}= & \frac{\mathrm{w}}{\mathrm{x}_{\mathrm{o}}} \quad \rho \leq 1 \\
\text { Where } \quad \rho_{\mathrm{s}}= & \text { Specific resistivity } \\
\mathrm{x}_{\mathrm{O}}= & \begin{array}{l}
\text { Absolute thickness, by Tolansky } \\
\text { interferometer }
\end{array} \\
\mathrm{w}= & \begin{array}{l}
\text { Calculated thickness, by sheet } \\
\text { resistivity }
\end{array}
\end{aligned}
$$

\section{Apparatus}

2. 1 Metallurgical microscope

Metalstar trinocular body, AO No. $2200 \mathrm{~T}-4$

American Optical Co.

(or equivalent)

2. 2 Polaroid camera, AO No. 1052A

American Optical Co.

(or equivalent)

2. 3 Interferometer jig

Fairchild design 
2. 4 Monochromatic light source

2. 4. 1 Mercury, $5461 \AA$

LaPine Scientific Co. Model 216-01

(or equivalent)

2. 4.2 Sodium, $5893 \AA$ (optional)

LaPine Scientific Co. Mode1 215-96

(or equivalent)

2. 5 Four-point probe, 0.050 inch spacing

(any source)

2. 6 Power supply, constant current

0.1 , 1. 0, $10 \mathrm{~mA}$ ranges

(any source)

2.7 Voltmeter, Digital

Fairchild 7100

(or equivalent)

2. 8 Hot plate, 6" $\times 6^{\prime \prime}$, electric

Temco, Type 1900

Corning, Pyroceram PC-35

(or equivalent)

2. 9 Polyethylene beaker

(any source)

3. Materials

3. 1 Hydrofluoric acid, $49 \%$ Reagent grade

3. 2 Acetic acid, glacial, Reagent grade

3. 3 Trichloroethylene, Electronic grade

3. 4 Acetone, Electronic grade

3. 5 Apiezon wax

3. 6 Slides, glass microscope, $1^{\prime \prime} \times 3^{\prime \prime}$ 


\section{Procedure}

4. 1 Preparation $\left(\frac{\mathrm{V}}{\mathrm{I}}\right)$

4. 1. 1 Process a polished test chip through the metallization operation to be evaluated.

NOTE. Form a step in the film either by etching or "shadowing" with a glass slide during evaporation.

4. 2 Measurement $\left(\frac{\mathrm{V}}{\mathrm{I}}\right)$

4. 2. 1 Place the chip to be measured upon the probe stage. See Figure 1.

4. 2.2 Lower the probe until it touches the sample lightly.

4. 2. 3 Record the voltmeter reading.

4.2. 4 If the current source is $100 \mu \mathrm{A}, 1 \mathrm{~mA}, 10 \mathrm{~mA}$, etc., the voltmeter will read directly in sheet resistance with application of the proper decimal point.

4.2. 5 Using the formula below, calculate the film thickness. Example: $\frac{V}{I}=0.006 \Omega$

Film - aluminum

$$
\begin{aligned}
0 & =2.72 \times 10^{-6} \Omega-\mathrm{cm}, 25^{\circ} \mathrm{C} \\
w & =\frac{\rho \ln 2}{\pi \frac{V}{I}} \\
& =\frac{2.72 \times 10^{-6} \times .693}{\pi \times .006} \\
& =\frac{2.72 \times 10^{-6} \times 6.93 \times 10^{-1}}{\pi \times 6 \times 10^{-3}} \\
& =\frac{2.72 \times 6.93 \times 10^{-4}}{\pi \times 6} \\
& =1 \times 10^{-4} \mathrm{~cm} \\
& =1 \mathrm{micron} \\
w & =10^{4} \AA
\end{aligned}
$$


4. 3 Preparation (Tolansky interferomete $r$ )

4.3. 1 Evaporate a $1000 \AA$ silver or aluminum film over the sample.

NOTE: While silver has a higher index of reflectivity, it must not be allowed to tarnish prior to measurement.

4. 4 Measurement (Tolansky interferometer)

4. 4. 1 Place the specimen on the platform, mirrored side up. See Figure 2.

4.4.2 Rotate the specimen on the platform until the step to be measured is normal to the front edge of the Fizeau plate.

4. 4.3 Raise the platform until the specimen is in contact with the Fizeau plate by turning the knurled brass elevation nut. This will give maximum fringe spacing.

4. 4. 4 If a smaller fringe spacing is desired, the platform may be raised slightly, reducing the wedge angle, $\theta$, and, as a result, fringe spacing. See Figure 3.

4. 4. 5 Make a photomicrograph.

4. 4.6 Measure distance between fringes on the photomicrograph with a precision rule (W). Figure 4.

4. 4.7 Measure the distance which any fringe is displaced as it passes across the film step. (D)

4. 4.8 Calculate film thickness, $\mathrm{x}_{\mathrm{o}}$, using the relationship:

$$
\frac{\mathrm{x}_{\mathrm{o}}}{\mathrm{D}}=\frac{\frac{\lambda}{2}}{\mathrm{w}}
$$




$$
\text { Example: } \begin{aligned}
\text { Mercury light, }=5460 \AA \\
\mathrm{W}=0.350 \mathrm{inch} \\
\mathrm{D}=1.50 \mathrm{inch} \\
\mathrm{x}_{\mathrm{o}}=\frac{2730}{0.350} \times 1.50 \\
\mathrm{x}_{\mathrm{o}}=11700 \AA
\end{aligned}
$$

4. 5 Resistivity calculation

4. 5. 1 Using the electrically and visually determined thickness, specific resistivity may be calculated as:

$$
\begin{aligned}
\rho_{s} & =\frac{w}{x_{o}} \\
\text { Example: } \quad w & =10,000 \AA \\
x_{o} & =11,700 \AA \\
\rho_{s} & =\frac{10,000}{11,700} \\
\rho_{s} & =0.85
\end{aligned}
$$

\section{Calibration}

\section{1 Probe spacing}

5. 1. 1 Cover a $1 " \times 3$ " glass microscope slide with aluminum foil.

5. 1. 2 Obtain an impression of the four probe points.

5. 1.3 Measure spacing with any optical comparator.

5. 1. 4 Adjust spacing per probe manufacturer's instructions. 
6. Accuracy

Accuracy obtainable is approximately $\pm 20 \%$.

7. Test Frequency

This test should be performed on a periodic basis. 
Method 145

FOUR POINT PROBE - SET-UP

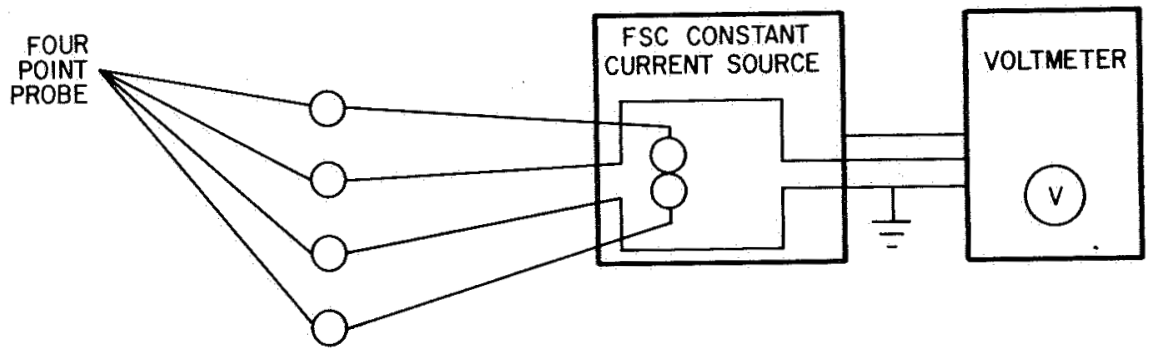

Figure 1. 


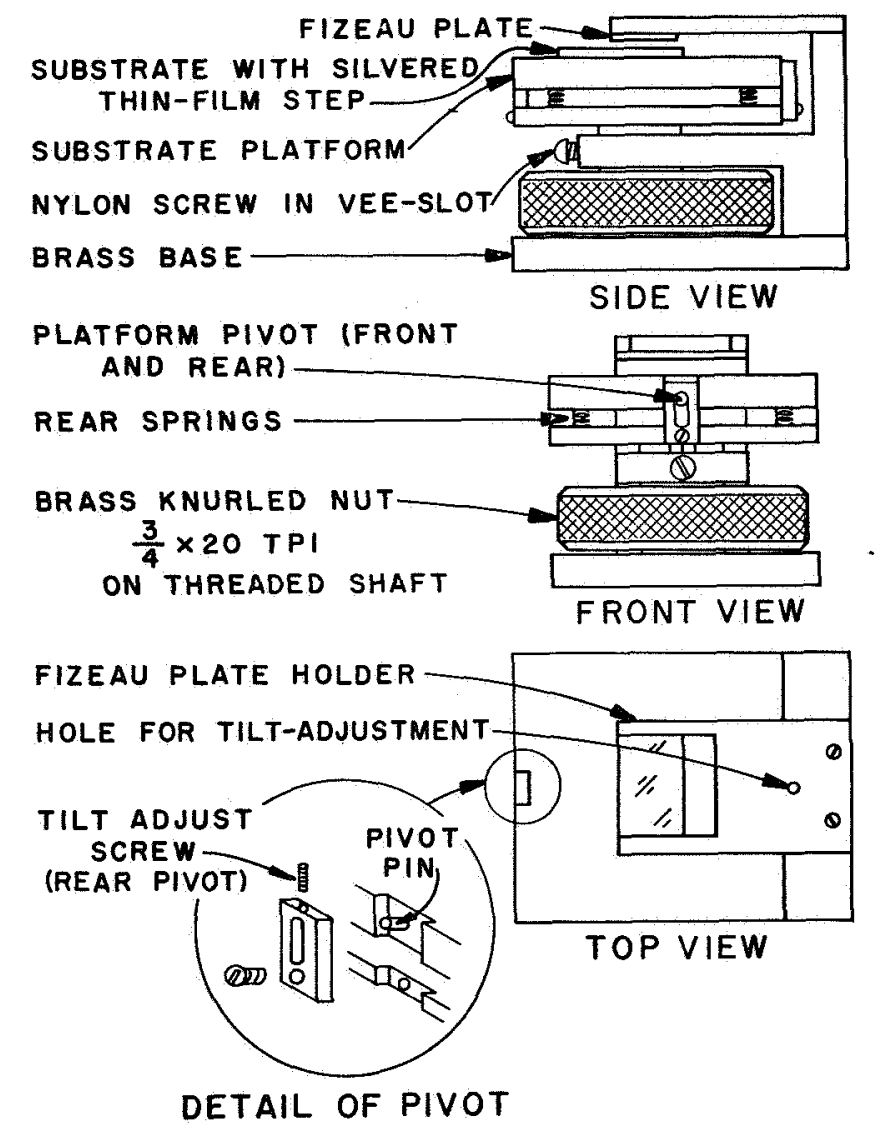

Figure 2. Interferometer Jig 


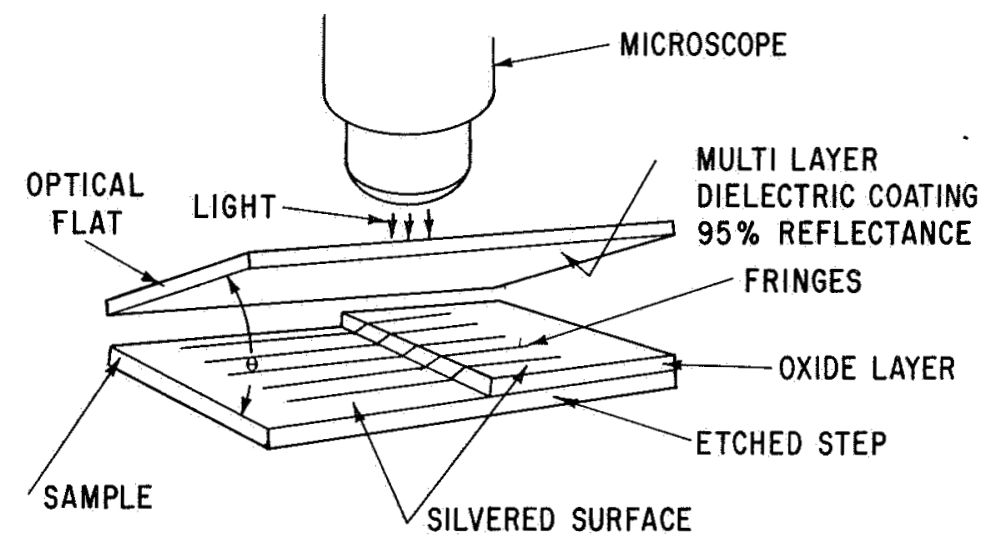

Figure 3. Tolansky Interferometer Setup 


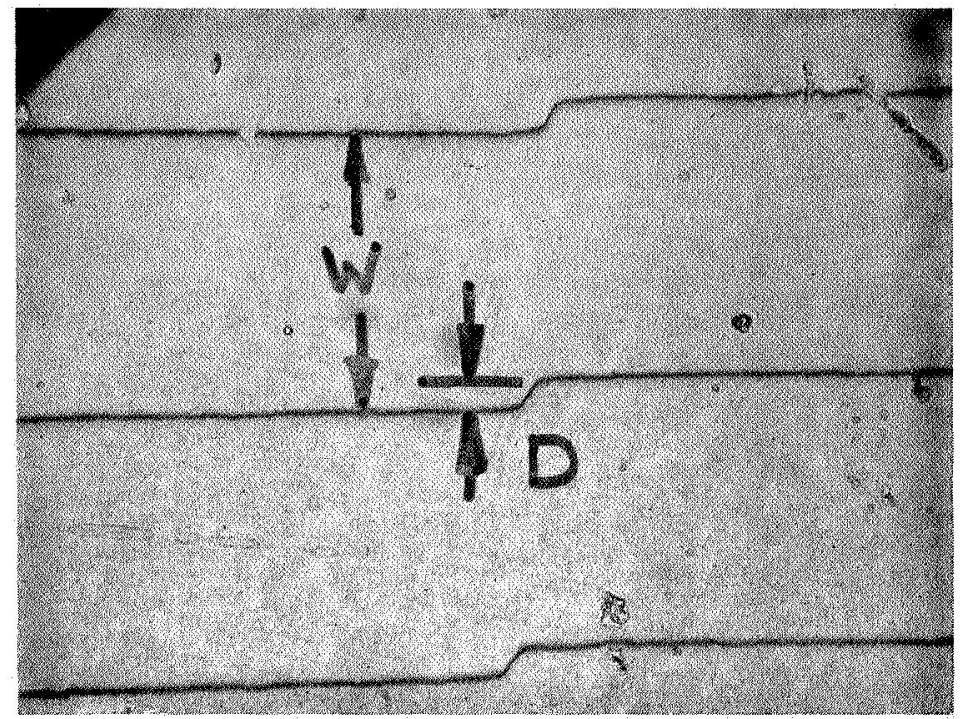

Figure 4. Film Measurement

$$
\begin{aligned}
& \text { Sodium, } \lambda=5890 \AA \\
& \mathrm{W}=1.12 \text { inches } \\
& \mathrm{D}=0.14 \text { inches } \\
& \mathrm{X}_{\mathrm{O}}=\mathrm{D} \frac{\frac{\lambda}{2}}{\mathrm{~W}} \\
& =0.14 \frac{\frac{5890}{2}}{1.12} \\
& =\frac{1.4 \times 2.945 \times 10^{2}}{1.12} \\
& =3.4 \times 10^{2} \\
& =340 \AA
\end{aligned}
$$

NOTE: A fixed error of $30-50 \AA$ becomes significant at this thickness range. 
Method 146

Quality of Ohmic Contact

1. Purpose The purpose of this test is to determine that an ohmic contact exists between aluminum interconnections and the diffused structure. It employs evaluation of a series of diffused resistors.

2. Apparatus

2. 1 Transistor Curve Tracer

Fairchild 6200

Tektronix 575

(or equivalent)

2. 2 Two-point Probe with micro-manipulator (any source)

3. Materials

3. 1 Silicon wafer with diffused and metallized test pattern. Figure 1 .

\section{4. $\quad$ Procedure}

4. 1 Obtain wafer with test pattern after metallization alloy.

4. 2 Place wafer upon the probe stage. Figure 2.

4. 3 Probe one pad with the emitter probe and one with the collector probe.

4. 4 The oscilloscope trace should be a straight line through the origin with a finite slope. Figure 3 .

4. 5 A vertical line (short) indicates excessive alloying and a horizontal line indicates either a rectifying contact or an open. 
5. Calibration

5. 1 Calibrate the curve tracer per manufacturer's instructions.

5. 2 A typical trace should be obtained by placing a $1-10 \mathrm{~K} \Omega$ resistor across the probes.

6. Accuracy

Adjustment of current and voltage scales to deliver a $45^{\circ}$ slope will provide maximum accuracy.

7. Test Frequency

Each wafer should be sampled at final wafer test. 


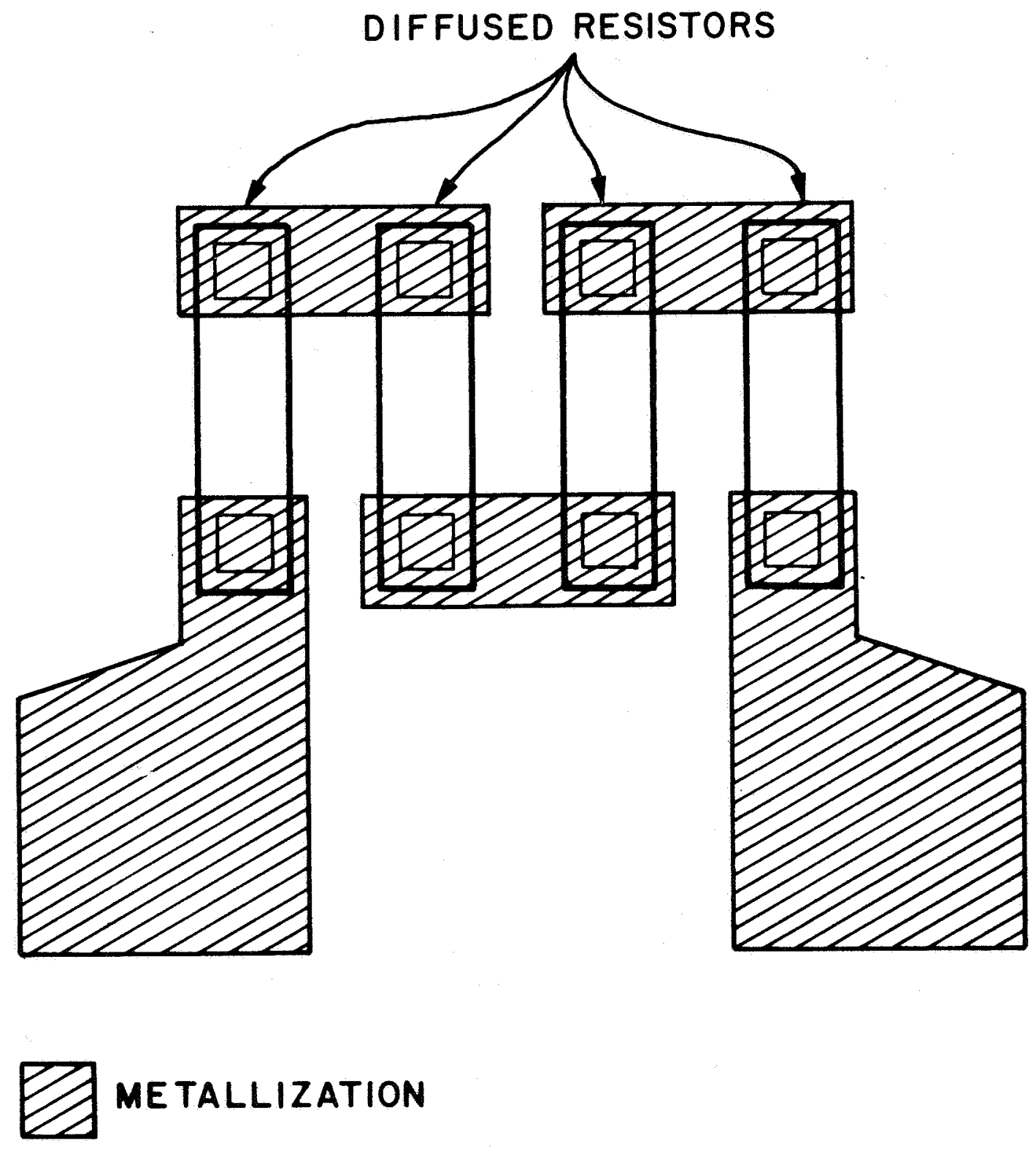

Figure 1. Test Pattern 


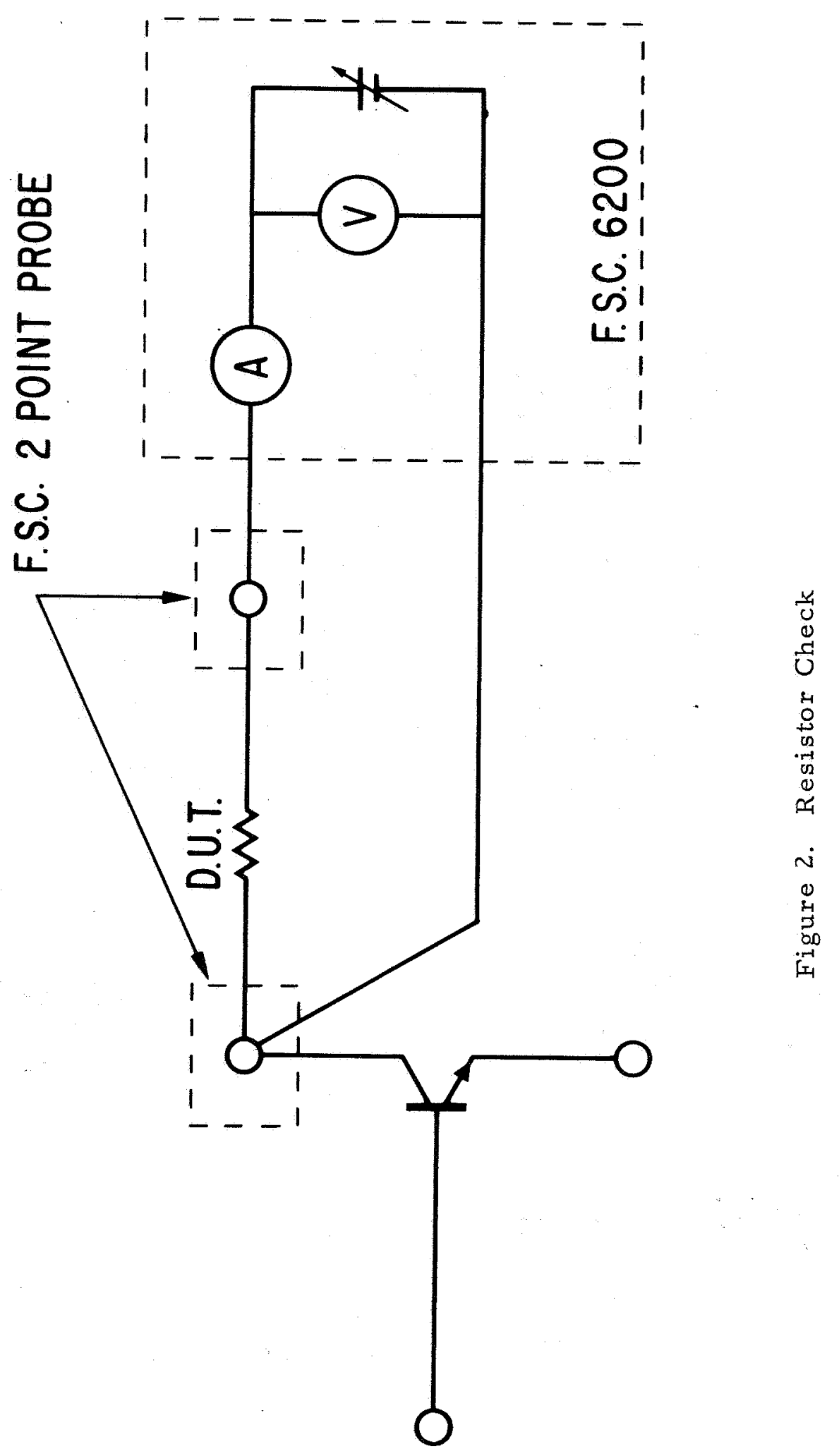


Method 146

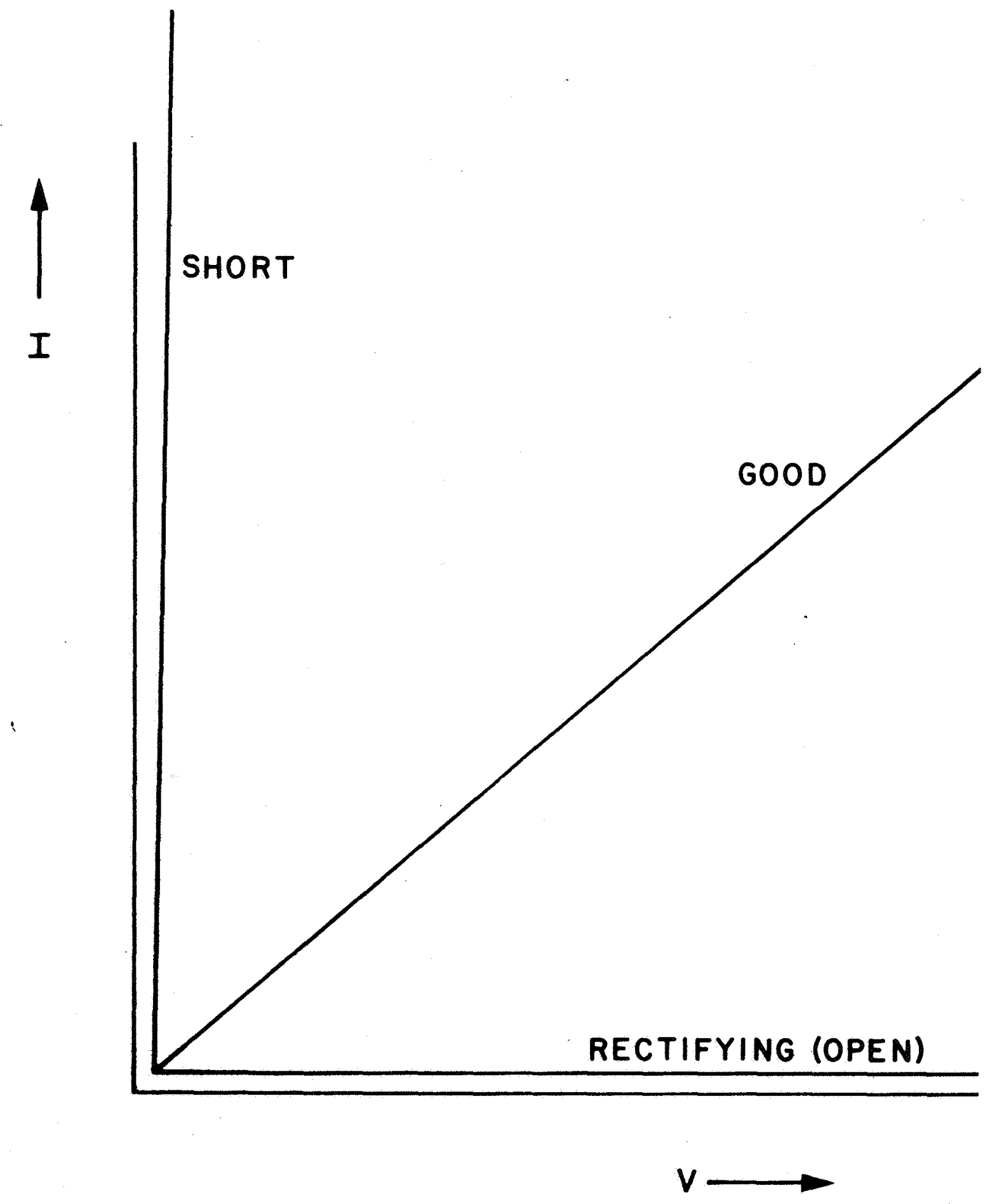

Figure 3. Oscilloscope Trace 


\author{
Method 147 \\ Cross Sectional Uniformity \\ of Metallization
}

1. Purpose The purpose of this test method is to evaluate the uniformity of cross section of the metallized interconnections and bonding pads.

2. Apparatus

2. 1 Metallurgical Microscope

Bausch and Lomb 31-20-68-38 (DMETR)

(or equivalent)

3. Materials

3. 1 Glass microscope slides, $1^{\prime \prime} \times 3^{\prime \prime}$.

4. Procedure

4. I Place the alloyed, metallized wafer upon a clean glass slide on the microscope stage.

4. 2 Adjust the magnification to $150 \mathrm{x}$ and scan the wafe $\mathrm{r}$ for the following defects:

4.2. 1 Geometry - Adherence to design dimensions and shape.

4.2. 2 Scratches - Scratches which reduce the designed cross-sectional area.

NOTE: A scratch with oxide, substrate, etc., showing through will usually be deeper and more severe than one with metal covering the bottom of the damaged area. Figure 1.

4. 2. 3 Alignment - Registration of metallized interconnections with the passivating layer cut-outs. Figure 2. 
4.2. 4 Undercutting - Failure of the metallizationphotoresist interface resulting in the width at the interconnection top being significantly narrower than the width in contact with the passivated substrate. Figure 3.

4.2. 5 Voids - Holes in the metallization film which significantly reduce the cross-sectional area either by their individual size or density.

4.2.6 Cut-Out Continuity - Cracking, narrowing or discernible thinning of interconnections at the passivating layer cut-out. Figure 4.

5. Calibration.

Not applicable.

6. Accuracy

6. 1 Scratches and cracks greater than 1 micron.

6. 2 Voids greater than 2 microns.

6.3 Geometry alignment and undercutting within designed tolerance.

7. Test Frequency

Every wafer should be sampled prior to the dicing operation and all circuits should be inspected. 

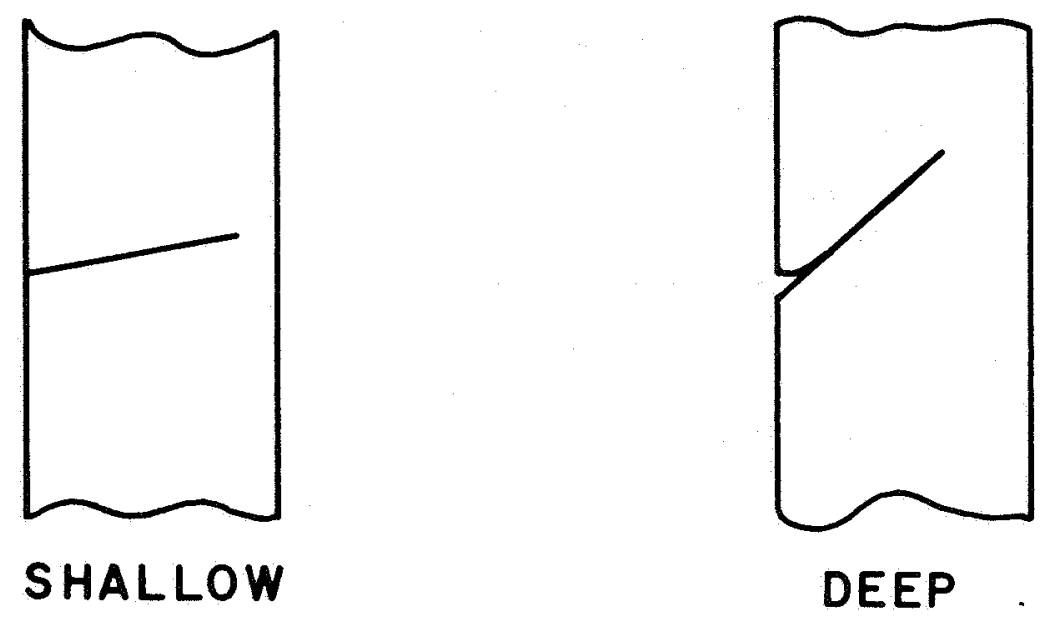

Figure 1. Interconnection Scratches

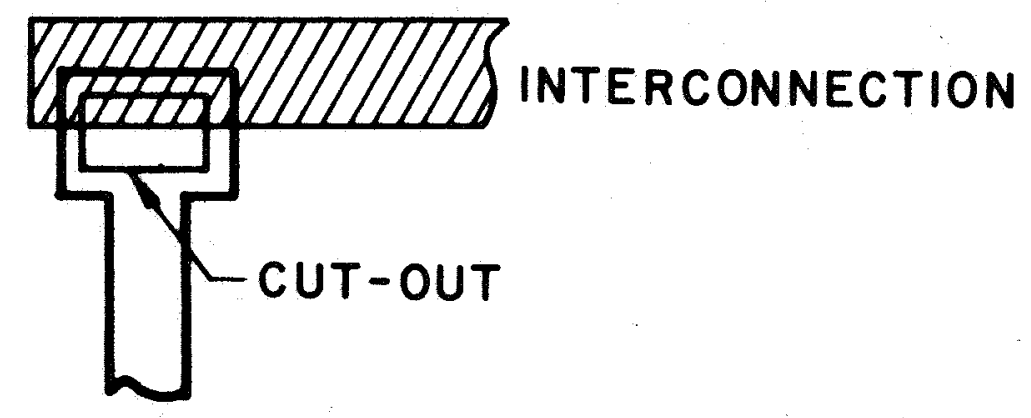

Figure 2. Alignment 


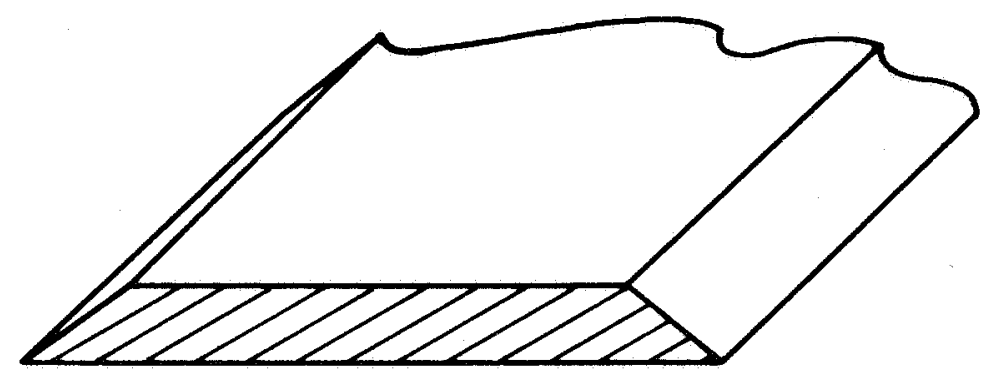

NORMAL

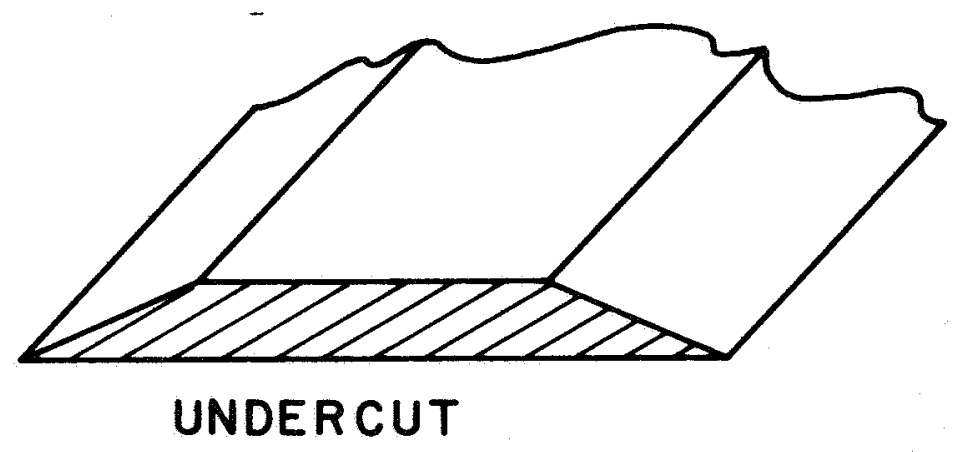

Figure 3. Interconnection Undercutting 


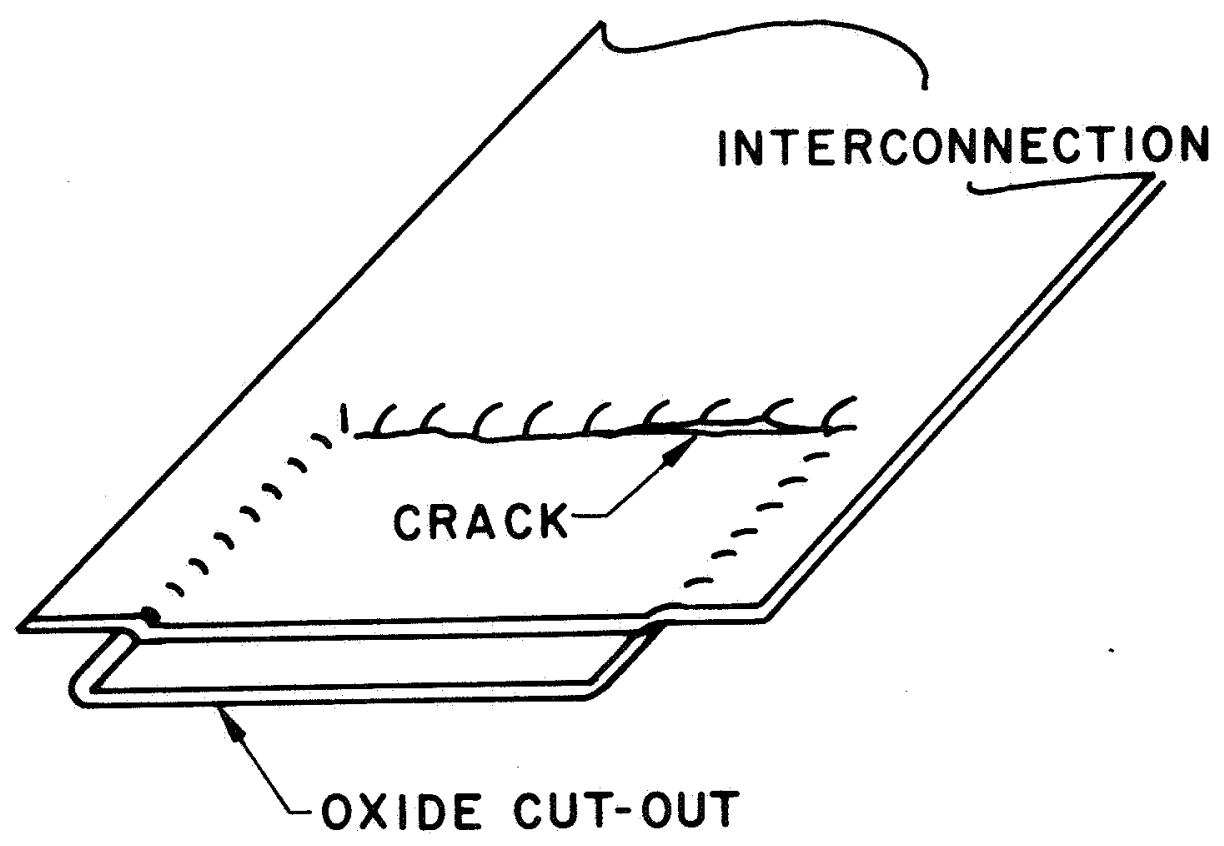

Figure 4. Cut Out Continuity 
Method 148

Pull Strength of Interconnection Bonds

1. Purpose This method may be used to evaluate wire-wire bond system. It treats the die bond, package bond and connecting wire as one component.

2. Apparatus

2. 1 Pull Test Fixture. See Figures 1 and 2.

(or equivalent)

2.2 Gram Gauge

ARPO Gram Gauge (0-25 grams), Scherr-Tunico Distributor (or equivalent)

2. 3 Binocular Microscope, 45X

(any source)

3. Materials

None.

4. $\quad$ Procedure

4. 1 Adjust heater block to desired temperature $\left(25^{\circ}-125^{\circ} \mathrm{C}\right)$ and allow to stabilize.

NOTE: Production monitoring will normally be at room temperature.

4. 2 Place adapter on heater block for package to be tested (Flat Pak, TO-5, etc). 
4. 3 Focus microscope on lead system.

4. 4 Insure recorder hand of gram gauge is at zero.

4. 5 Using the micromanipulator, position the small wire hook under the arch of the bond system to be tested.

4.6 Raise the gram gauge slowly until the bond system fails. This will be caused by one of the following:

4.6.1 Wire - Wire parts due to excessive tension.

4.6.2 Light Bond - Bond is lifted, intact, from bonding area.

4.6.3 Broken Bond - Bond fails, leaving residue of wire on bonding area.

4. 7 Repeat Paragraph 4.4 to 4.6 for all bonds to be evaluated.

4. 8 Note reading and failure mode.

\section{Calibration}

5. 1 Monitor elevated temperature with a chromel-alumel thermocouple attached to the proper package, in a method similar to die-attach temperature measurement.

5. 2 Check the gram gauge by hanging a 5 or 10 gram laboratory weight from the lever arm ("paddle") and noting correlation.

\section{Accuracy}

Accuracy obtained will be \pm one small division of the gram gauge.

\section{Test Frequency}

All bonding stations should be monitored periodically. 
Method 148

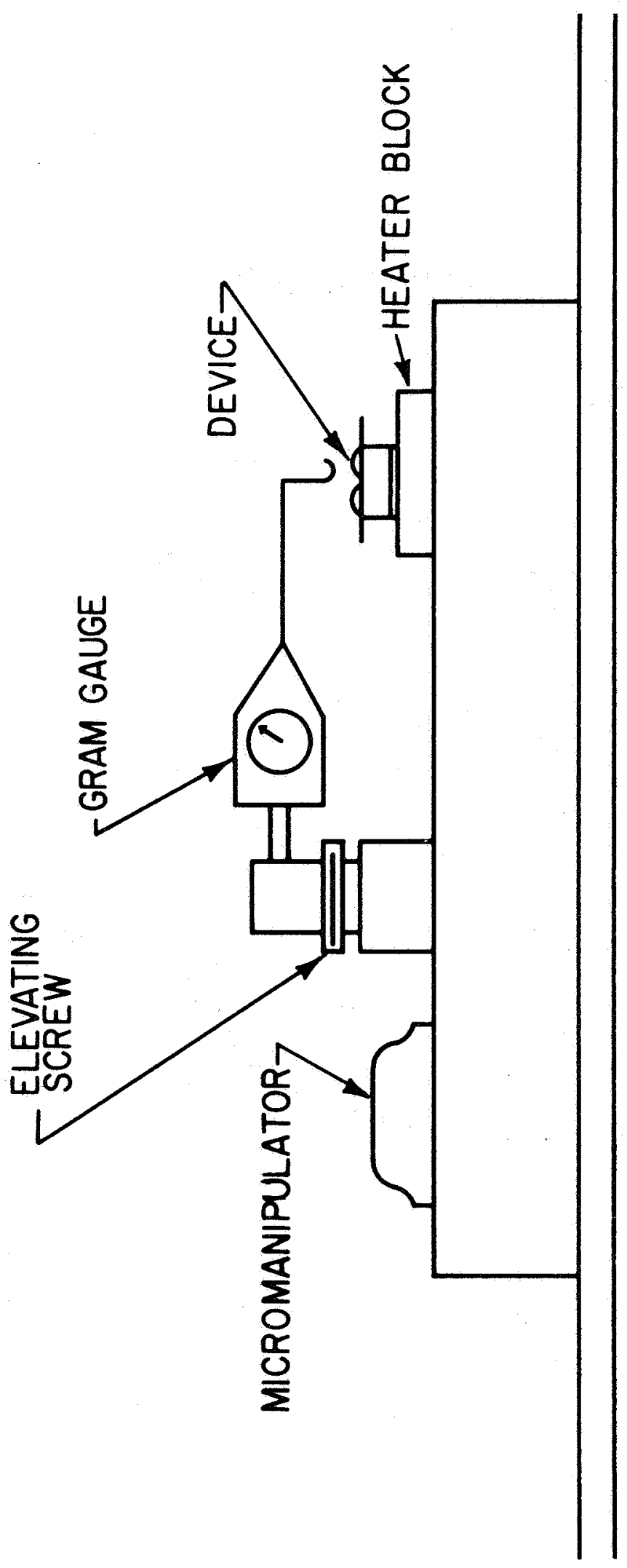

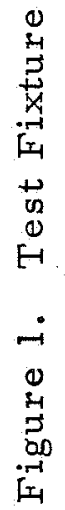



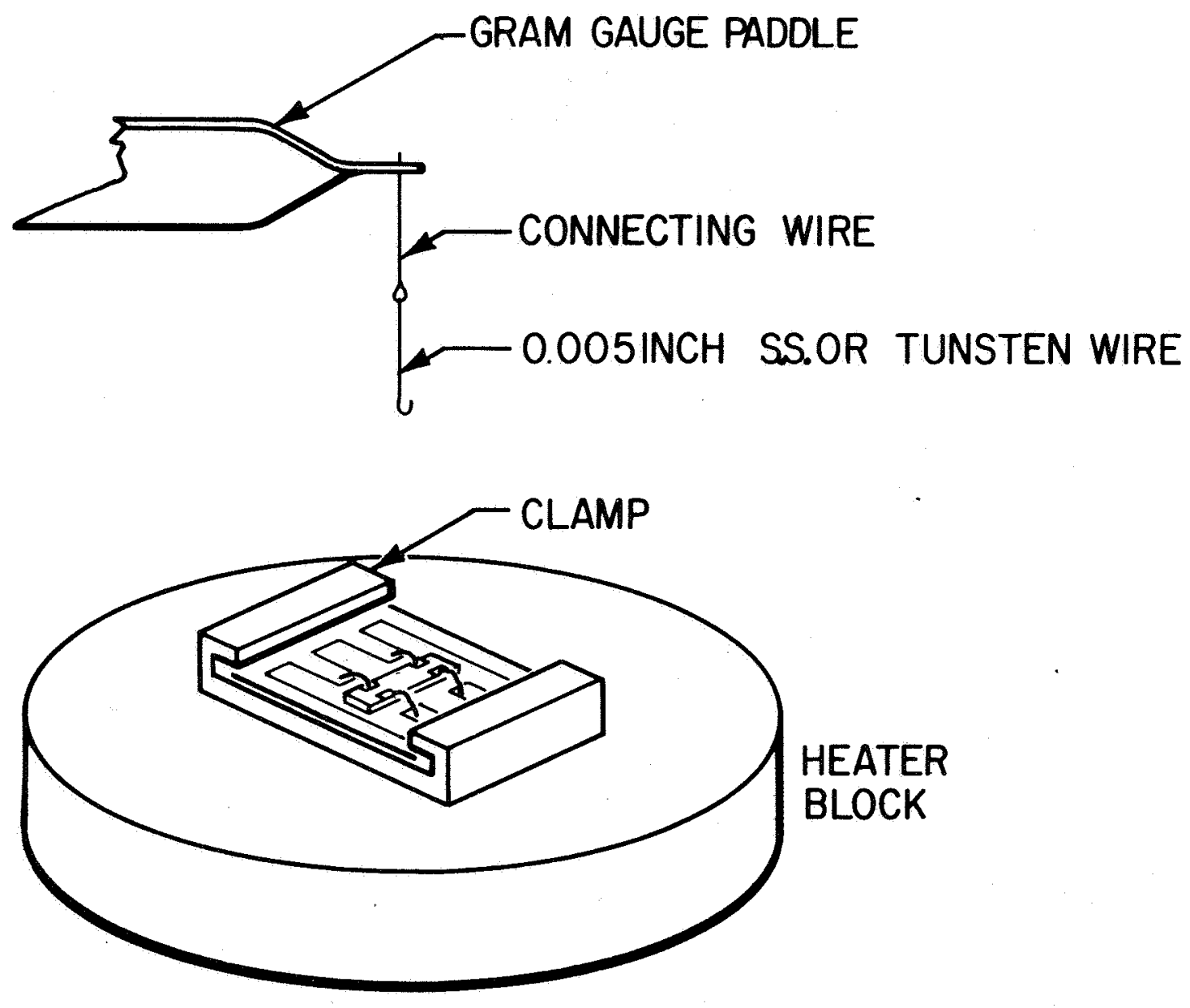

Figure 2. Test Detail 


\section{Method 149}

Moisture Content

(Dry Box Sealing)

1. Purpose This test is applicable to the determination of moisture content of the ambient trapped during dry-box sealing operations.

\section{Apparatus}

2. 1 Moisture Monitor

Model 26-301 or 26-303

Consolidated Electrodynamics Corp.

(or equivalent)

2. 2 Diaphragm Pump

Dyna-Pump Model 2

(or equivalent)

\section{Materials}

None.

\section{4. $\quad$ Procedure}

4. 1 The moisture monitor should be connected to sample the dry-box and entry chamber as shown in Figure 1.

4. 2 Set by-pass flow at 600-800 cc. per minute.

4. 3 Adjust sample flowmeter to exactly $50 \mathrm{cc}$ per minute.

4. 4 By use of the appropriate valves, connect the moisture monitor with the location to be sampled.

4. 5 Turn the scale selector to "100x" and allow 5 minutes for stabilization.

4. 6 If the needle has less than one-third of full scale deflection, turn selector to the next lowest scale. 
4. 7 Repeat 4.6 until maximum definition. is obtained.

4. 8 Multiply scale reading by the selector factor for moisture content in parts per million (by volume). See Figure 2.

\section{Calibration}

Calibration should be as noted in the manufacturer's instructions.

6. Accuracy

Accuracy obtainable should be $\pm 10 \%$ at about 100 PPM.

\section{Test Frequency}

Moisture content of the dry-box should be monitored during sealing and at least the following times:

7. 1 Entry, prior to introducing parts into dry-box.

7.2 Dry-box after opening to admit parts

7. 3 Dry-box, after discharging sealed units.

7. 4 Line cases, to verify dryness. 


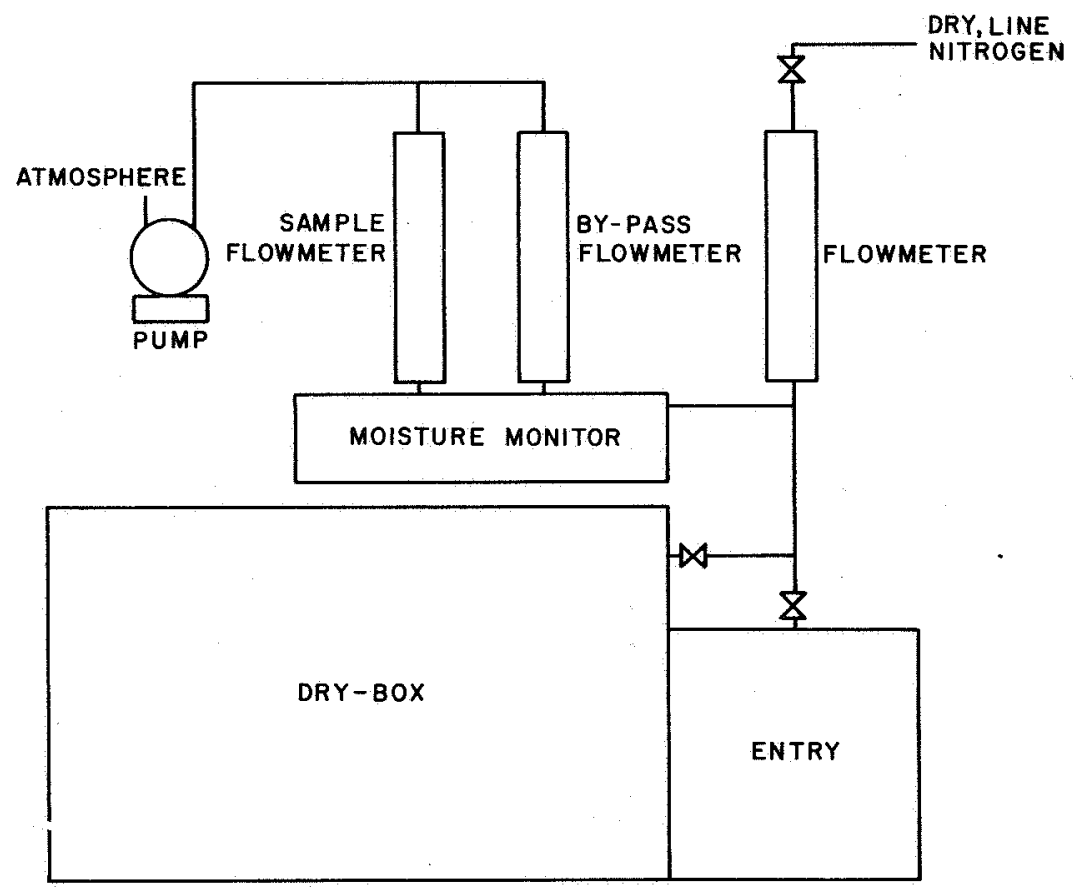

Figure 1. Moisture Monitor Connections 


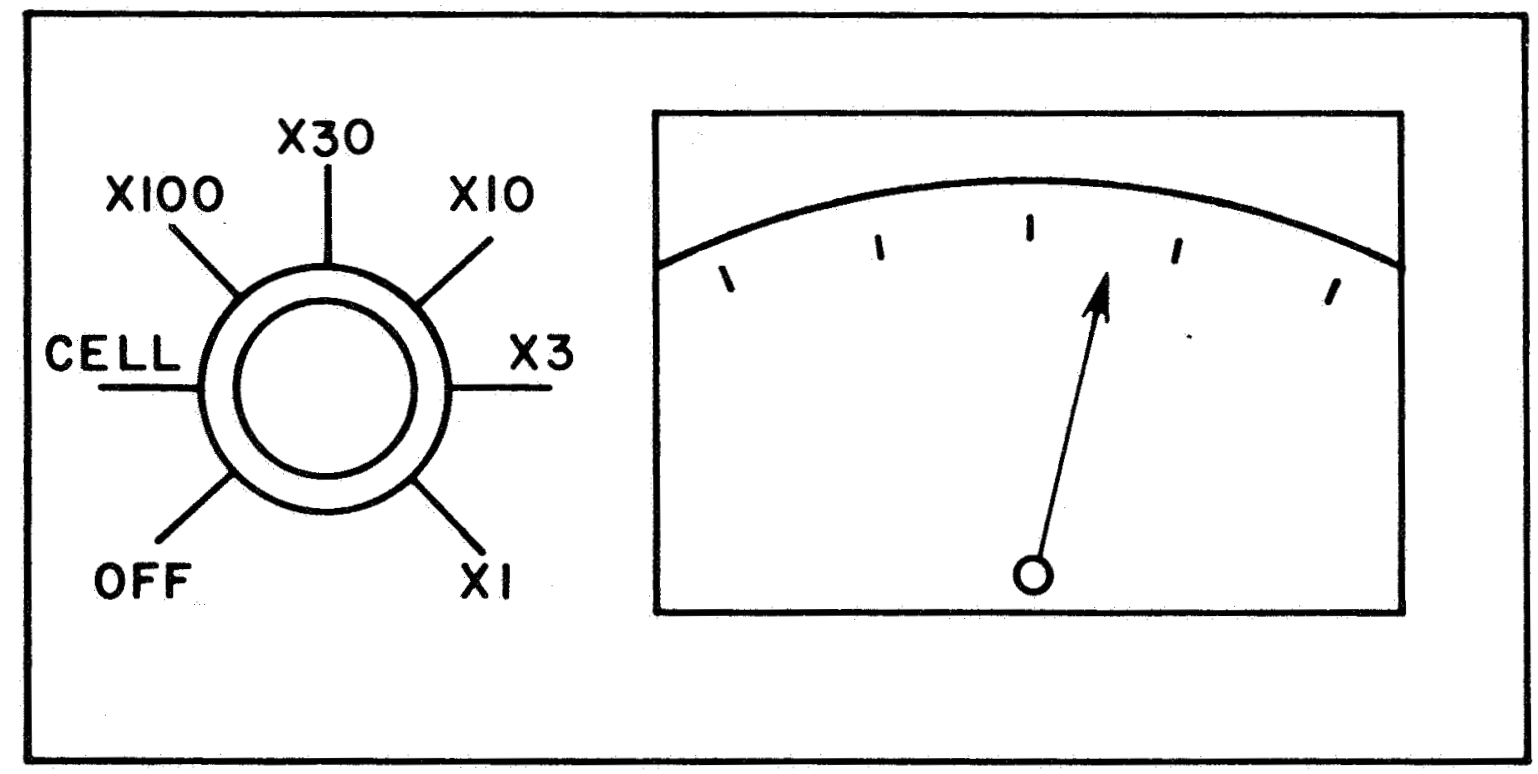

Figure 2. Moisture Monitor Scale 


\author{
Method 150 \\ Moisture Content \\ (Belt Furnace Sealing)
}

1. Purpose The purpose of this test is to determine the moisture content of gases enclosed in the device package. This method is particularly applicable to belt furnace operations. It utilizes the formation of visible water vapor in an observation chamber under known conditions which establishes moisture content.

2. Apparatus

2. 1 Dew Point Monitor

Dew Pointer Type 7000

Alnor Instrument Company

(or equivalent)

3. Materials

3. 10.25 inch I. D. Neoprene Tubing

3. $26 \mathrm{~mm}$ Quartz Tubing

4. Procedure

4. 1 Place a length of quartz tubing into the sealing a rea of the furnace.

4. 2 Connect the quartz tube to the dew point monitor inlet with a short length of Neoprene Tubing.

NOTE: For dew points below $-20^{\circ} \mathrm{F},(422 \mathrm{PPM})$ the connection should be via a brass ball-and-socket adapter lubricated with silicone vacuum grease. Figure 1.

4. 3 Connect the monitor to a 115 volt A-C receptacle.

4. 4 Open the purging valve. Figure 2.

4. 5 Pull out the operating valve. 
4. 6 Turn the power switch "ON".

4. 7 Pump the gas to be tested through the instrument to flush out all the old gas (at least 20 strokes of the pump.)

4. 8 Close the purging valve.

4. 9 Pump a sample of gas into the monitor.

4. 10 Note the pressure ratio.

4. 11 Look into the observation window and press down on the operating valve.

4. 12 If a fog appears, it indicates the gas was cooled below the dew point. Make the subsequent trials (repeat steps 4. 10 to 4.12 ) at higher pressure ratios (lower pressures) until one reading is obtained where no fog appears. The last fogged reading is the "End Point".

4. 13 If no fog appears on the first trial, the gas was not cooled down to the dew point. Subsequent trials should be made at lower pressure ratios (higher pressures).

4. 14 Note the "End Point".

4. 15 Determine the " $Q$ " value of the gas from Table I. NOTE: " $Q$ " as applied, represents the cooling power for a given gas.

4. 16 Note the temperature.

4. 17 Calculate the dew point using the circular slide rule provided with the dew point monitor.

4. 18 Enter Table II with the gas dew point and determine moisture content in parts per million (by volume).

\section{Calibration}

Prior to each use the instrument must he adjusted to unity pressure ratio as follows: 
5. 1 Place operating valve in the out position.

5. 2 Open purging valve.

5. 3 Depress the gauge valve and turn the unity adjuster until the bottom of the miniscus is in line with the "l" mark.

5. 4 Release the gauge valve.

\section{Accuracy}

Accuracy should be $\pm 20 \%$ at dew point below $-40^{\circ} \mathrm{F}$.

\section{Test Frequency}

This test should be performed periodically on belt type furnaces.

"Q" Values of Various Gases

\begin{tabular}{|lc|}
\hline Air & .285 \\
Argon & .400 \\
Carbon Dioxide & .225 \\
Helium & .400 \\
Hydrogen & .285 \\
Nitrogen & .285 \\
Oxygen & .285 \\
\hline
\end{tabular}

Table I 
Dew Point to p. p. m. Conversion Table

\begin{tabular}{|c|c|c|c|c|c|c|c|c|c|c|c|}
\hline D.P. & ppm & D.P. & ppm & D.P. & ppm & D.P. & ppm & D.P. & ppm & D.P & ppm \\
\hline $\begin{array}{l}-130 \cdot \mathrm{F} \\
-120 \\
-110 \\
-105 \\
-104 \\
-103 \\
-102 \\
-101 \\
-100 \\
=99 \\
=98 \\
-97 \\
-96 \\
-95 \\
=94 \\
-93 \\
-92\end{array}$ & $\begin{array}{l}0.1 \\
0.25 \\
0.63 \\
1.00 \\
1.08 \\
1.18 \\
1.29 \\
1.40 \\
1.53 \\
1.66 \\
1.81 \\
1.96 \\
2.15 \\
2.35 \\
2.54 \\
2.76 \\
3.00\end{array}$ & $\begin{array}{r}-91^{\circ} \mathrm{F} \\
-90 \\
-89 \\
-88 \\
-87 \\
-86 \\
-85 \\
-84 \\
-83 \\
-82 \\
-81 \\
-80 \\
-79 \\
-78 \\
-77 \\
-76 \\
-75 \\
-74\end{array}$ & $\begin{array}{l}3.28 \\
3.53 \\
3.84 \\
4.15 \\
4.50 \\
4.78 \\
5.3 \\
5.7 \\
6.2 \\
6.6 \\
7.2 \\
7.8 \\
8.4 \\
9.1 \\
9.8 \\
10.5 \\
11.4 \\
12.3\end{array}$ & $\begin{array}{l}-73^{\circ} \mathrm{F} \\
-72 \\
-71 \\
-70 \\
-69 \\
-68 \\
-67 \\
-66 \\
-65 \\
-64 \\
-63 \\
-62 \\
-61 \\
-60 \\
-59 \\
-58 \\
-57\end{array}$ & $\begin{array}{l}13.3 \\
14.3 \\
15.4 \\
16.6 \\
17.9 \\
19.2 \\
20.6 \\
22.1 \\
23.6 \\
25.6 \\
27.5 \\
29.4 \\
31.7 \\
34.0 \\
36.5 \\
39.0 \\
41.8\end{array}$ & $\begin{array}{r}-56^{\circ} \mathrm{F} \\
-55 \\
-54 \\
-53 \\
-52 \\
-51 \\
-50 \\
-49 \\
-48 \\
-47 \\
-46 \\
-45 \\
-44 \\
-43 \\
-42 \\
-41 \\
-40 \\
-39\end{array}$ & $\begin{array}{l}44.6 \\
48.0 \\
51 \\
55 \\
59 \\
62 \\
67 \\
72 \\
76 \\
82 \\
87 \\
92 \\
98 \\
105 \\
113 \\
119 \\
128 \\
136\end{array}$ & $\begin{array}{l}-38^{\circ} \mathrm{F} \\
-37 \\
-36 \\
-35 \\
-34 \\
-34 \\
-33 \\
-32 \\
-31 \\
-30 \\
-29 \\
-28 \\
-28 \\
-27 \\
-26 \\
-25 \\
-24 \\
-23 \\
-22\end{array}$ & $\begin{array}{l}144 \\
153 \\
164 \\
174 \\
185 \\
196 \\
210 \\
222 \\
235 \\
250 \\
265 \\
283 \\
300 \\
317 \\
338 \\
358 \\
378\end{array}$ & $\begin{array}{l}-21^{\circ} \mathrm{F} \\
-20 \\
-19 \\
-18 \\
-17 \\
-16 \\
-15 \\
-14 \\
-13 \\
-12 \\
-11 \\
-10 \\
=9 \\
=8 \\
-7 \\
=6 \\
-5 \\
-4\end{array}$ & $\begin{array}{r}400 \\
422 \\
448 \\
475 \\
500 \\
530 \\
560 \\
590 \\
630 \\
660 \\
700 \\
740 \\
780 \\
820 \\
870 \\
920 \\
970 \\
1020\end{array}$ \\
\hline
\end{tabular}

Table II 
Method 150

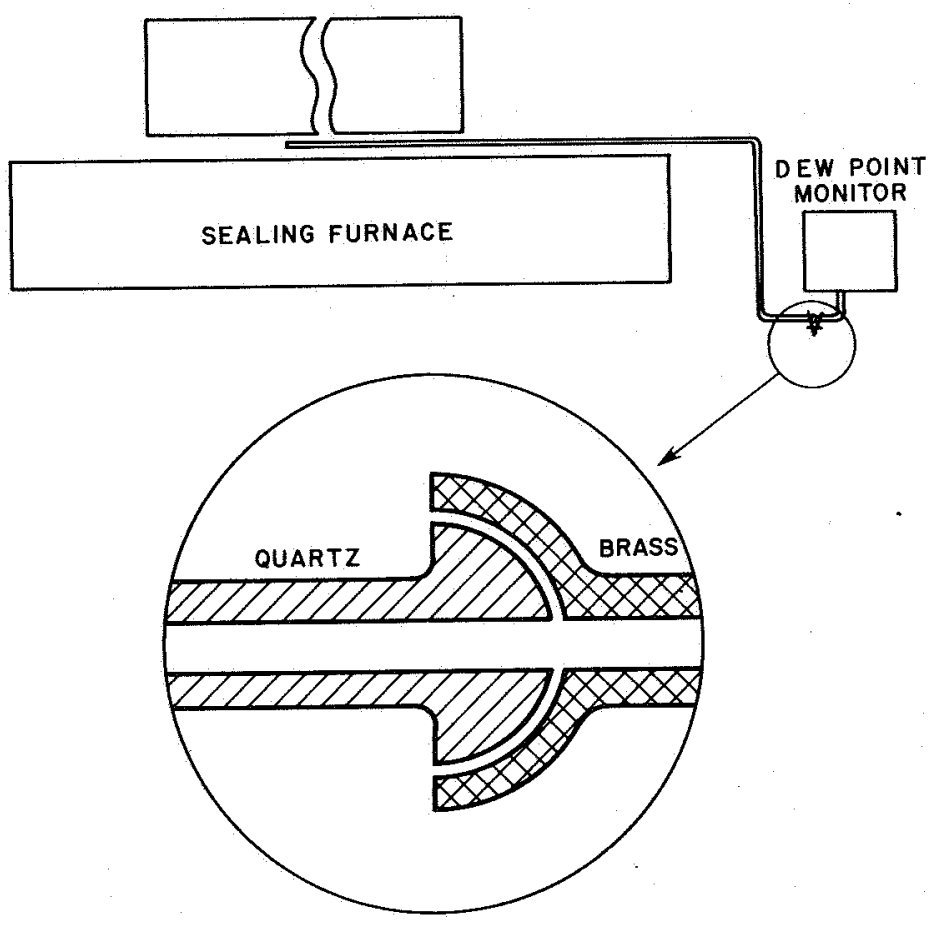

Figure 1. Dew Point Monitor Setup 
Method 150
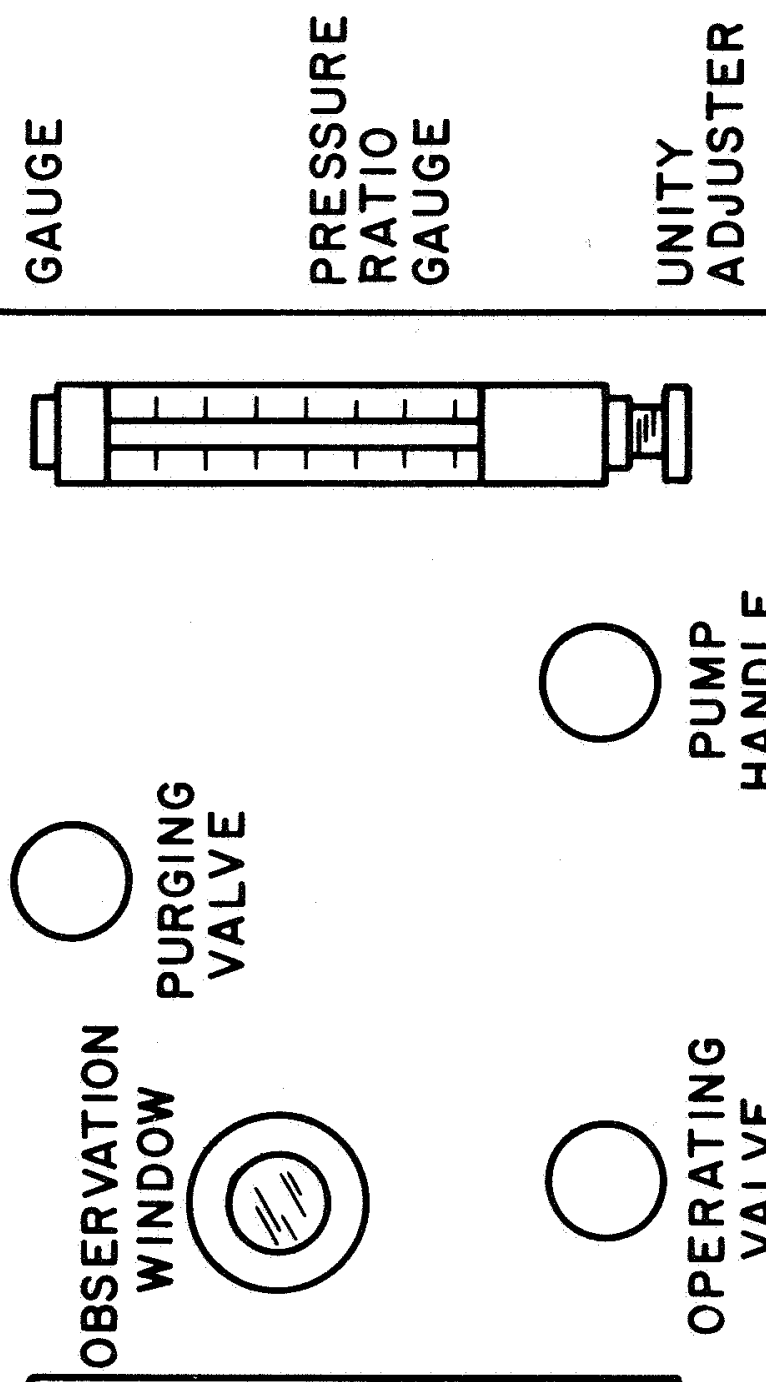

○敦

$w$
$w$
$w$
$\sum$
0
$\alpha$
$w$
$I$ 
Method 151

\section{Hermaticity, Fine and Medium Leak (Radiflo)}

1. Purpose

The purpose of this test is to detect fine and medium leaks $\left(10^{-9}\right.$ to $\left.10^{-6} \mathrm{cc} / \mathrm{sec}\right)$ in the package of any device with an internal cavity. It utilizes the injection of radioactive gas into defective units and detection by a scintillation counter.

2. Apparatus

2. 1 Radiflo Activation Unit

Consolidated Electrodynamics Corp., Model C2D5

(or equivalent)

2. 2 Geiger-Mueller Counter

Consolidated Electrodynamics Corp.

(or equivalent)

2. 3 Scintillation Counter

Consolidated Electrodynamics Corp.

(or equivalent)

2. 4 Scintimonitor

Consolidated Electrodynamics Corp.

(or equivalent)

2. 5 Survey Meter

Universal Atomic Division, Universal Transistor Products Corp., Model SM-160

(or equivalent) 
3. Materials

3. 1 Krypton-85

4. Procedure

4. 1 Activation

4. 1. 1 Presetting Activation Pressure and Activation Time

4. 1. 1. 1 Find the inspection level of the leak rate called for in the controlling documents $\left(1 \times 10^{-9}, 1 \times 10^{-8}, 1 \times 10^{-7}\right.$, or $\left.1 \times 10^{-6}\right)$. Find the Activation Pressure and Activation Time corresponding to this leak rate in the manufacturer's operating manual.

4. 1. 1. 2 Record Activation Pressure and Activation Time in the appropriate columns of the Radiflo Operation Log.

4.1.1.3 Go to the open portion of the plastic covered panel at the lower left of the machine. Preset the Activation Pressure by turning the knob under the Pressure Indicator until the needle points to the pressure value obtained in 4.1.1.1.

4. 1. 1. 4 Preset the Activation Time obtained by turning the Set knob, located in the lowe $r$ left of the open portion of the plastic cover, until the correct value appears on the mileage-type meter.

4. 1. 1. 5 If the Activation Time required for a given load of devices is the same as for the load just processed, 4.1.1.4 may be omitted by simply pressing the Reset button.

4. 1.2 Loading the Test Units

4. 1.2. 1 Load the devices into the wire basket. 
4. 1.2. 2 Read Storage Activity Meter by holding Storage Monitor button depressed for at least 20 seconds and record value in Radiflo Operation Log. Read Storage Pressure Meter and record value in Radiflo Operation Log.

4. 1. 2. 3 Push the Door button and lift hinged door.

4. 1. 2. 4 Unlock lid of the Activation Tank by pulling the handle out.

4. 1.2. 5 Depress the two Open buttons to raise the lid of the Activation Tank.

4. 1.2. 6 Wipe the bottom of the lid with industrial tissue to remove any particles of dirt.

4. 1.2. 7 Insure that the 0-ring on top of the Activation Tank is free of dust.

NOTE: At the beginning of each day shift, the 0 -ring shall be removed, wiped clean with industrial tis sue, lightly lubricated with vacuum grease and reinserted. This cleaning operation shall be noted in the Radiflo Operation Log.

4. 1.2.8 Lower the basket of devices into the Activation Tank.

4. 1. 2. 9 Depress the two Close buttons to close the Activation Tank.

4. 1. 2. 10 Push handle back to seal lid.

4. 1. 2. 11 Shut hinged door.

4. 1. 2. 12 Press Evacuate button to initiate the Radiflo cycles of:

(1) Evacuate

(2) Activate

(3) Store

(4) Wash 
4. 1.3 Unloading the Test Units

4. 1. 3. 1 After the Radiflo has completed its cycles (indicated by the Stop and Door buttons lighting up) repeat 4.1 .2 .2 through 4.1.2. 5 of the Loading procedure.

4. 1.3.2 Remove the basket of devices from the Activation Tank.

4. 1.3.3 If more devices are to be activated, place them in the Activation Tank as soon as possible and repeat the activation process.

4. 1.3. 4 If no more devices a re immediately available for activation, close the lid of the Activation Tank, seal the lid, and shut the hinged door.

4. 1.3. 5 It is imperative that devices are inspected in the Scintimonitor within two hours after removal from the Radiflo. Devices must be reactivated if they are not inspected within this interval.

4. 1.3.6 DO NOT LEAVE LID OPEN AND UNSEALED.

\section{2 Detection}

For purpose of testing, the operator must translate the $\mathrm{cc} / \mathrm{sec}$ failure leak rate to counts/minute by referring to the manufacturer's operating manual.

4.2. 1 Turn Master switch to "ON" and allow equipment to warm for two minutes.

4.2. 2 Turn $\mathrm{H}-\mathrm{V}$ (High-Voltage) switch to "ON'.

4.2.3 Turn Function switch to zero. Adjust the center knob in the upper left of the console panel until the meter needle points to zero.

4.2. 4 Turn Function switch to "CALIBRATE." Turn the $\mathrm{C} / \mathrm{M}$ (Counts/Minute) knob to $1 \mathrm{~K}$. Adjust the outer knob in the upper left of the console panel until the needle reads between the two vertical red lines on the upper scale of the meter. 
4.2. 5 Turn Function switch to $\mathrm{H}-\mathrm{V}$. Turn $\mathrm{H}-\mathrm{V}$ setting knob on upper right of console panel until the meter reads the voltage that has been posted on the panel just below the knob.

4.2.6 Turn Seconds knob to 2.0 on the black scale.

4. 2. 7 Turn Function switch to $\mathrm{C} / \mathrm{M}$.

4.2.8 The background count can now be read from the meter and should be approximately 400 counts / minute. (Do not wear a wrist watch with luminous parts while testing).

4. 2. 9 Place one of the standard reject units which are maintained at the console into the test tube. Insert the tube into the Scintimonitor. A reject reading on the meter indicates that the equipment is operating properly and that the operator may proceed with the test.

4.2. 10 Place enough devices into the test tube to fill it about half full. Insert the tube into the Scintimonitor.

4.2.11 Wait two seconds and observe the meter reading. If the counts/minute reading exceeds the allowable maximum as determined in 4.2 above, the test tube is known to contain one or more rejects. When this happens, the devices should be split into two groups and each group retested in the Scintimonitor. This process of dividing the group into smaller test samples should be continued until the reject(s) are isolated.

4.2.12 Place reject units in an evnelope. Write the device number, run number, operator badge number and the date on the envelope.

4.2.13 Safety Procedure.

4. 2. 13.1 Place envelope of reject units into lead safety box.

4.2.13.2 Store rejects in the Tank Room until the radiation has fallen to the background count of 400 counts/minute. 
5. Calibration

5. 1 See Paragraph 4.2.8 and 4.2.9 for background count and sample check.

5. 2 For all other calibration and maintenance, refer to manufacturer's manual.

6. Accuracy

Accuracy obtainable is no less $\pm 50 \%$ at all leak rates.

Example: Scintillation Count $=1 \times 10^{-6} \mathrm{cc} / \mathrm{sec}$. Actual rate will be between $5 \times 10^{-6}$ and $5 \times 10^{-5} \mathrm{cc} / \mathrm{sec}$.

\section{Test Frequency}

All sealed units should be tested. 


\author{
Method 152 \\ Hermaticity, Gross Leak \\ (Bubble Test)
}

1. Purpose This method may be used to detect gross leaks (on the order of $10^{-5}$ atmospheric cubic centimeters/second) in device packages.

2. Apparatus

2. 1 Hot Plate, Electric 6" $\times 6^{\prime \prime}$

Temco, Type 1900

(or equivalent)

2. 2 Magnetic Immersion Board

(any source)

2. 3 Pyrex Bowl

(any source)

2. 4 Thermometer, $200^{\circ} \mathrm{C}$

(any source)

3. Materials

3. 1 Mineral Oil, Clear

3. 2 Trichloroethylene, Electronic grade

4. Procedure

4. 1 Before loading the devices on the immersion board ensure that they are free of any foreign matter and that they are at room ambinet temperature. 
4. 2 Load the devices on the immersion board so that the major axis is in the horizontal position.

4. 3 Fill a Pyrex bowl of suitable size with clear mineral oil. Ensure that there is sufficient oil to cover the uppermost part of the devices to a minimum depth of one (1) inch.

4. 4 Place the bowl on the hot plate and turn the temperature control to $125^{\circ} \mathrm{C}$; monitor the temperature of the oil with a thermometer.

4. 5 After the temperature stabilizes at $125^{\circ}+5^{\circ} \mathrm{C}$, immerse the devices in the liquid and gently agitate until all trapped air is freed from the surface of the devices.

4. 6 After the freeing of trapped air, leave the devices immersed for a minimum of one (1) minute. Observe the devices, particularly the seal areas, for leaks which will be indicated by a flow of bubbles escaping to the surface of the mineral oil.

4. 7 Remove the devices from the immersion bath and wash in a beaker of TCE to remove all traces of the mineral oil.

4. 8 Segregate defective units from the good units.

4.9 Thoroughly dry the units on the warm air dryer or under the heat lamp before performing any additional tests.

5. Galibration

Not applicable.

6. Accuracy

Not applicable.

7. Test Frequency

All devices should be tested. 
Method 153

Sealing, Defects in Glass

(Can Package)

1. Purpose

The purpose of this method is to detect seal defects in the glass-to-metal seals of can-type packages.

2. Apparatus

2. 1 Binocular Microscope, 30X, with illuminator (any source)

\section{Materials}

None.

4. Procedure

4. 1 Inspect header prior to sealing under microscope.

4. 2 Rotate device so seal area is observed at various angles.

4. 3 Note the following defects:

4.3.1 Bubbles - A string of bubbles which connect one lead with another, Figure 1, or a pin with the eyelet, Figure 2 .

4.3.2 Void - Missing glass for the full length of the seal, either against the lead or completely in the glass. See Figure 3. 


\subsubsection{Glass Climb - Glass from seal flows an excessive distance, down lead or up pin.}

4.3. 4 Cracks - A fissure in the glass which connects one lead with another or a lead with the eyelet, similar to Paragraph 4.3.1

\section{Calibration}

Not applicable.

6. Accuracy

Not applicable.

\section{Test Frequency}

Each lot of headers should be sampled. 
Method 153

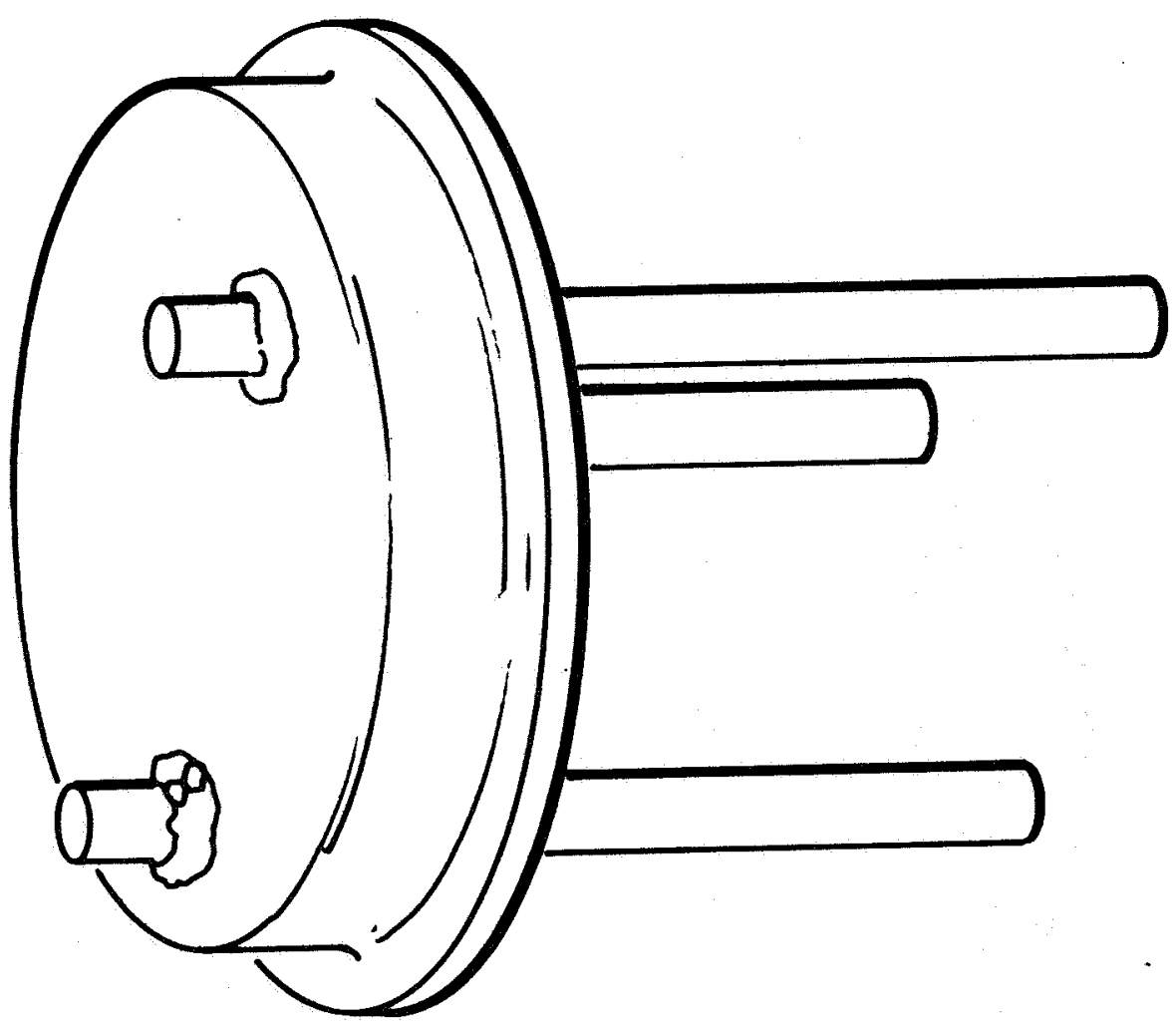

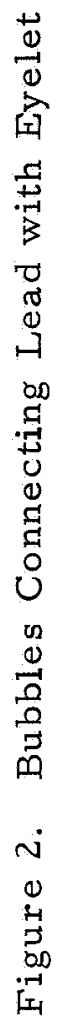

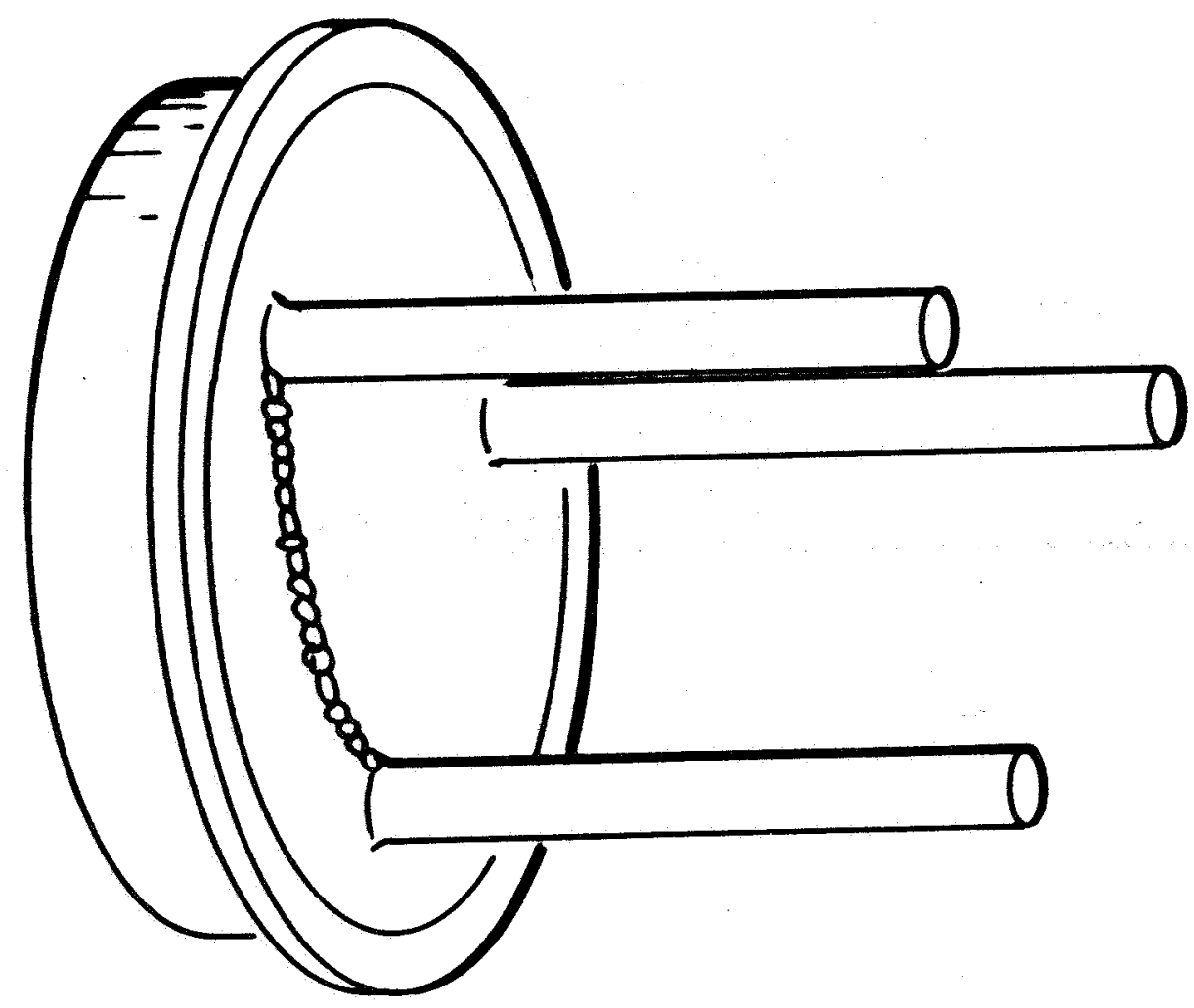

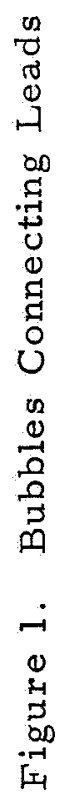


Method 153

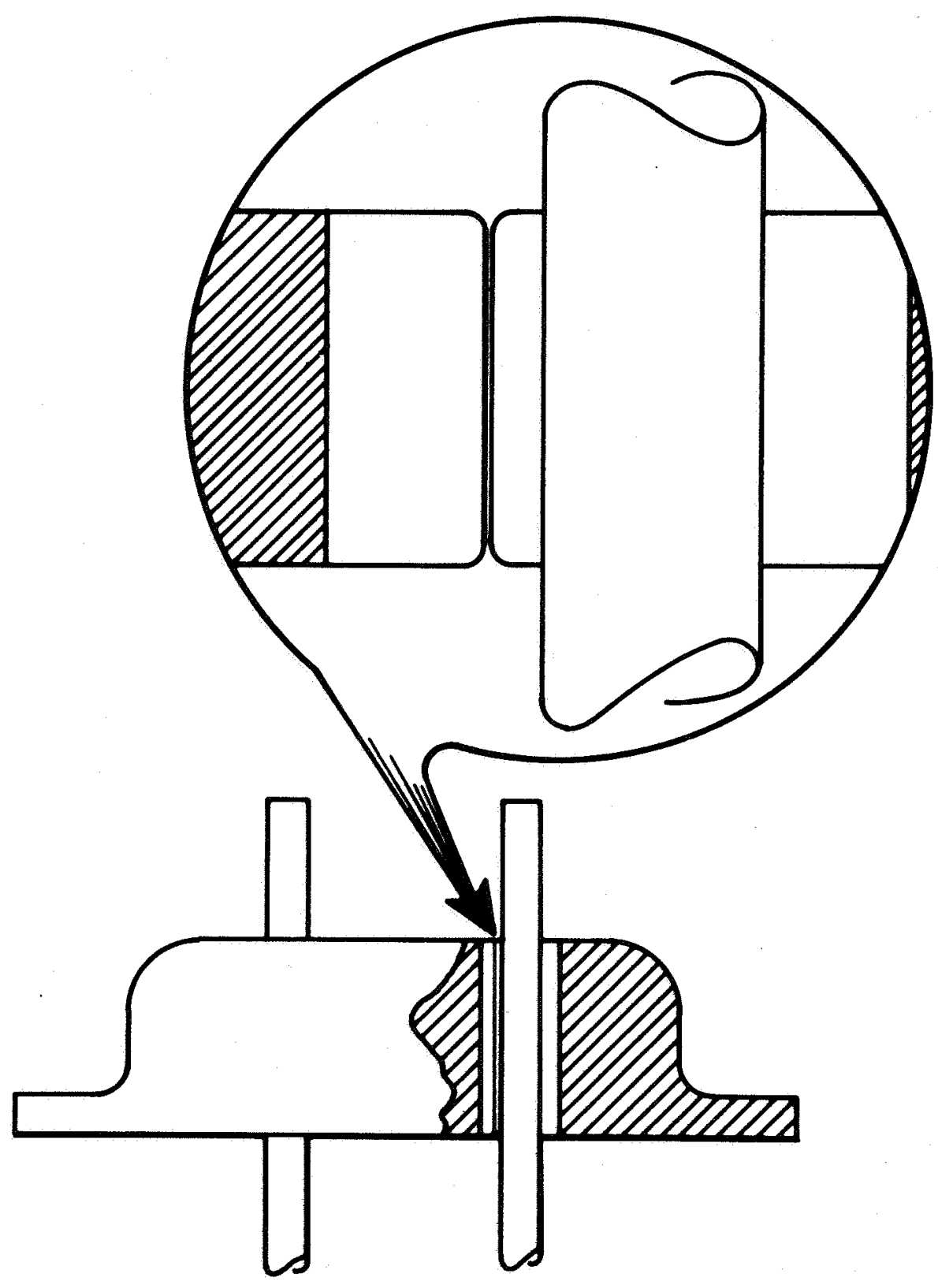

Figure 3. Illustration of Void 


\section{Method 154 \\ Sealing, Defects in Glass \\ (Flat Package)}

1. Purpose The purpose of this method is to detect seal defects in the glass seals of flat packages.

2. Apparatus

2. 1 Binocular Microscope, 15X, with illuminator (any source)

3. Materials

None.

4. $\quad$ Procedure

4. 1 Inspect sealed units under microscope.

4. 2 Rotate device so seal area is observed at various angles.

4. 3 Note the following defects:

4.3.1 Open Seal - Cap or base is tilted and glass does not make a complete seal. See Figure 1.

4.3.2 Sprung Seal - Cap cannot make a complete seal because frame leads are bent. See Figure 1. 
4.3.3 Insufficient Glass - Lack of glass in a distinct area to the degree that the bottom of the hole is not visible. See Figure 2 .

4. 3. 4 Pin Hole - A hole in the seal, without a visible bottom, less than one lead width in diameter. See Figure 3.

4. 3. 5 Blow Hole - Similar to Paragraph 4.3.4, but with surface residue. See Figure 3.

4.3.6 Warped Seal - An open seal due to warped ceramic. See Figure 4.

4.3.7 Glass Climb - Glass from seal flows out lead to an excessive distance.

4.3.8 Excess Glass - Package exceeds specified dimensions due to excess glass from seal.

\section{Calibration}

Not applicable.

6. Accuracy

Not applicable.

7. Test Frequency

All units should be tested after final sealing. 


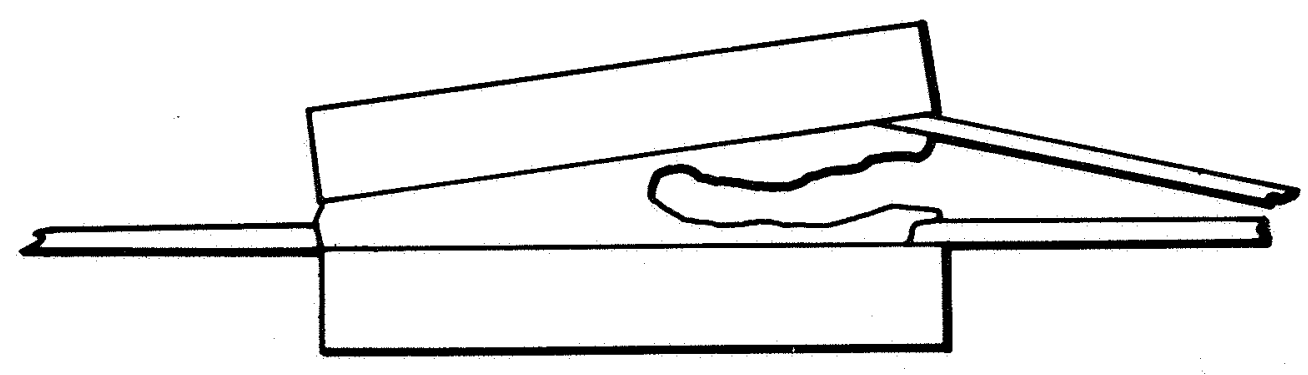

Figure 1. Open or Sprung Seal

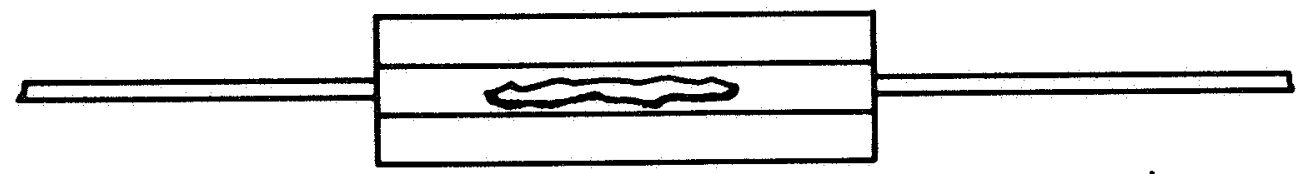

Figure 2. Insufficient Glass

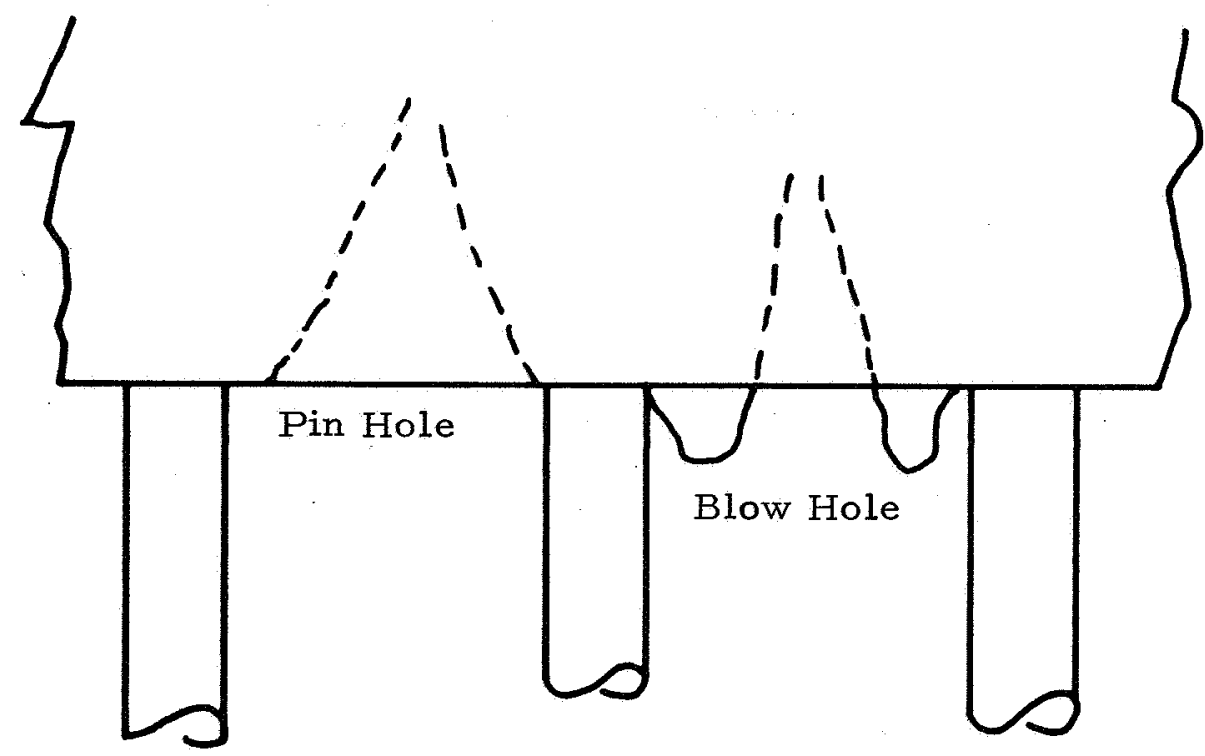

Figure 3. Holes in Glass 
Method 154
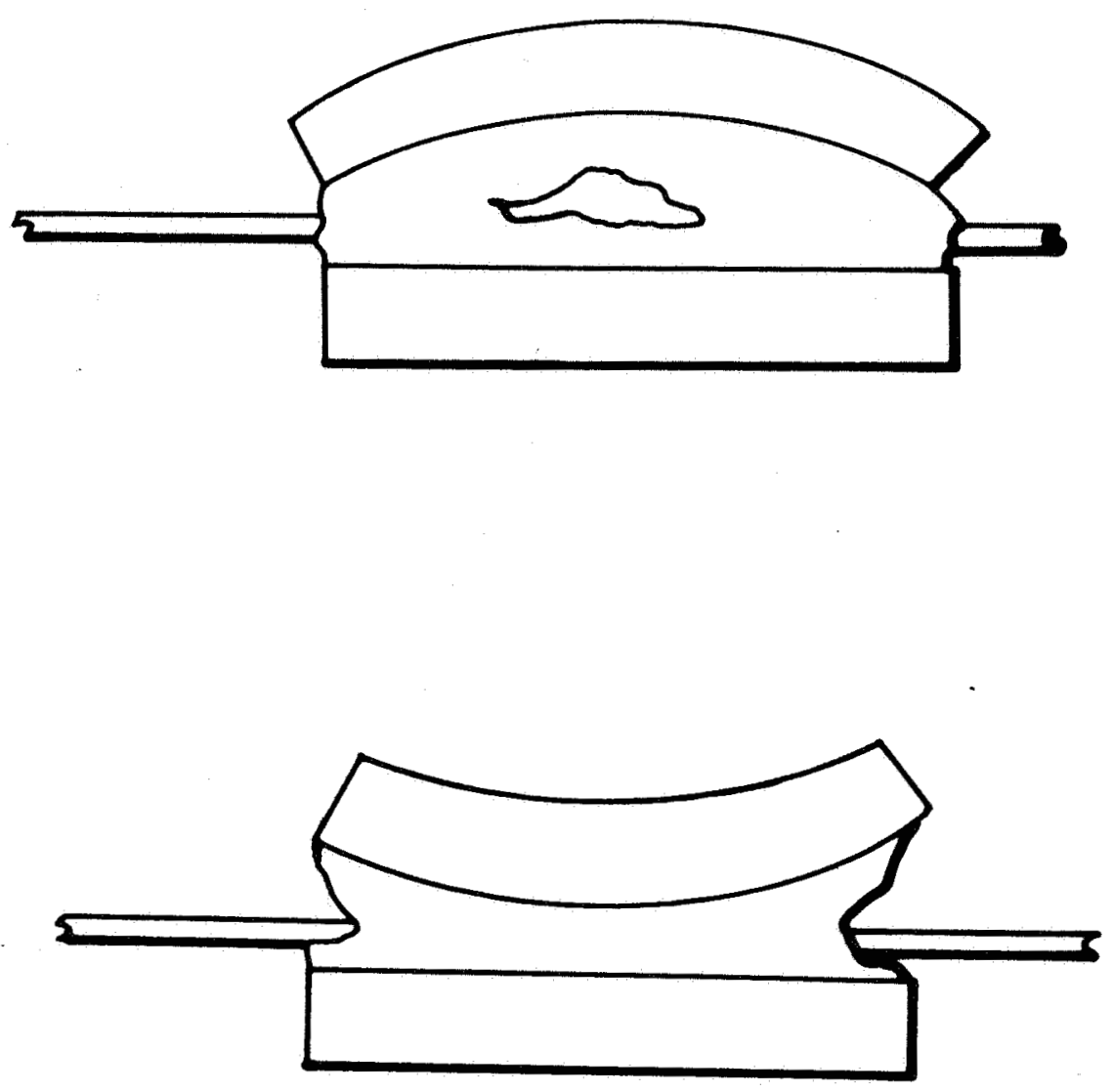

Figure 4. Warped Seal 


\section{Method 155}

Stored Charge, Bipolar Transistor $\left(\mathrm{Q}_{\mathrm{bx}}\right)$

1. Purpose The purpose of this test is to measure the stored charge of a device at specified conditions.

2. Test Circuit

See Figure 1.

3. Procedure

This procedure as depicted by Figure 1 fixes the input ' $Q$ ' and compares the variable ' $Q$ ' of the device in terms of storage time.

$$
\begin{aligned}
& Q=\frac{C V}{I} \\
& T s=\text { Storage time } \\
& T_{s}=\frac{Q C}{I C} \\
& Q=\text { Stored charge } \\
& \mathrm{C}=\text { Collector capacitance } \\
& \text { Ic }=\text { Collector current } \\
& \mathrm{C}_{(\text {in) }}=\text { Input capacitance } \\
& \frac{\mathrm{QC}}{\mathrm{IC}}=\frac{\mathrm{C}_{\text {(in) }} \mathrm{V}_{\text {(in) }}}{\mathrm{Ib}} \text { Where: } \mathrm{V}_{(\text {in) }}=\text { Input voltage } \\
& \text { Ib }=\text { Base current }
\end{aligned}
$$

Therefore, since the storage of a device is a function of $\mathrm{V}_{\mathrm{CE}}$ and collector capacitance, then with given conditions the stored charge may be obtained in Terms of Ts, using circuit in Figure 1. The specified ' $Q$ ' of the input, or comparison circuit, may be determined by changing the value of the input capacitance accordingly. 


\section{Summary}

The following details shall be specified.

(a) Input capacitance and resistances.

(b) Voltage and currents.

(c) Pulse conditions.

5. Accuracy

The accuracy of measurement will be dependent upon equipment used; $\left(Q_{b x}\right) \pm 3 \%$ with optimum equipment. 
Method 155

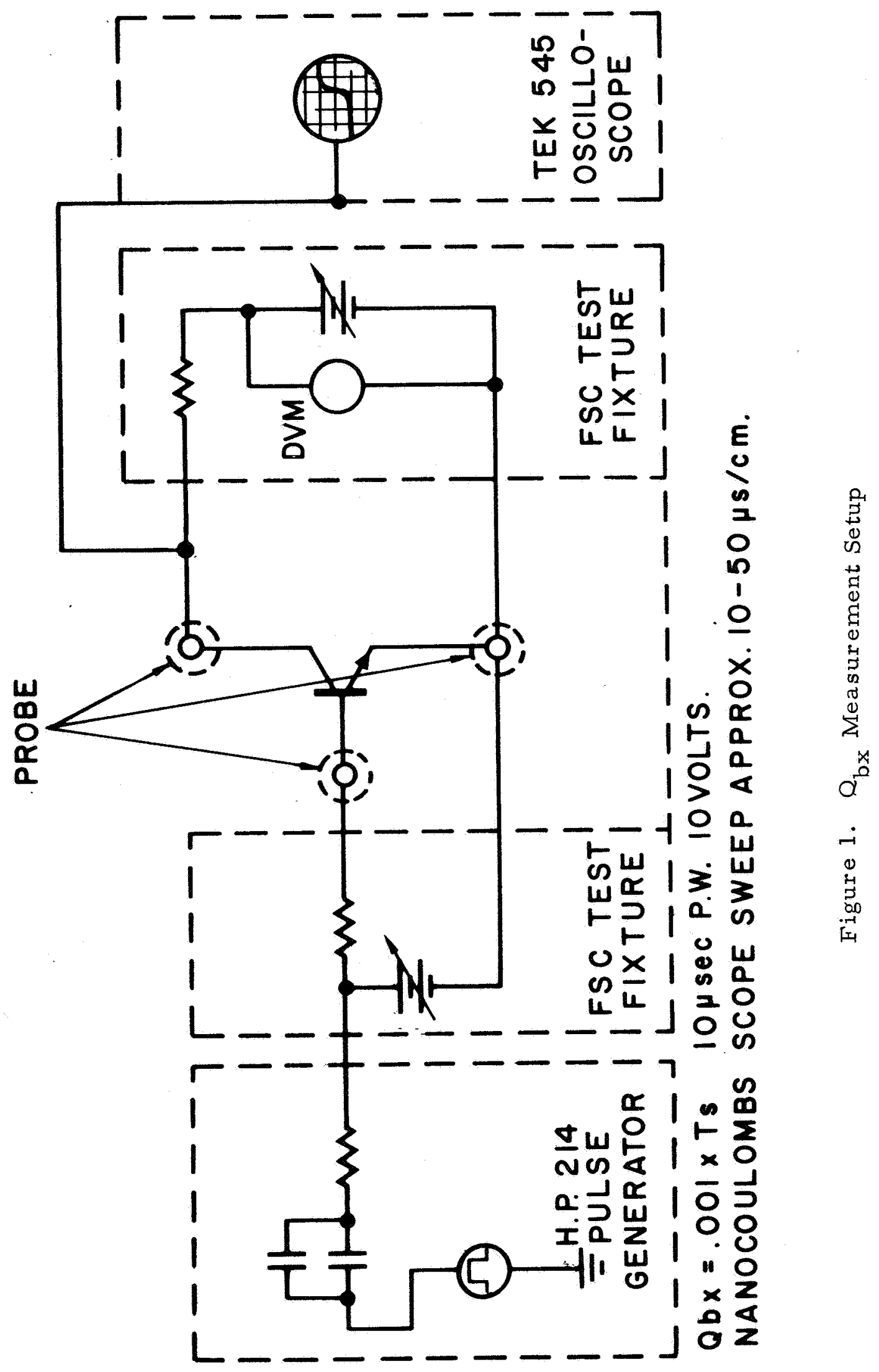




\section{Method 156 \\ Breakdown Voltage ( $\mathrm{BV}_{\text {DSS }}$ ) \\ Drain to Source}

1. Purpose The purpose of this test is to determine if the breakdown voltage of the device under the specified conditions is greater than the specified minimum limit.

2. Test Circuit

See Figure 1.

3. Procedure

The resistor $R_{1}$ is a current-limiting resistor and should be of sufficiently high resistance to avoid excessive current flowing through the device and current meter. The voltage shall be increased, with the specified bias conditions applied, until the specified test current is reached. The device is acceptable if the voltage applied at the specified test current is greater than the minimum limit for BVDS. If desirable, a suitable base current may be applied as the voltage is increased; however, the specified bias condition and test current must be applied when the voltage is measured. This method is intended for devices exhibiting negative resistance breakdown characteristics. In such cases, extreme care must be exercised to insure that the drain current and junction temperature of the device remain at a safe value. 


\section{Summary}

The following details shall be specified in the individual specification:

(a) Test current (See 3.)

(b) Duty cycle and pulse width, when required (See Note 1.)

(c) Bias condition as follows:

\section{A - Source to Gate - Short circuit.}

\section{Accuracy}

The accuracy of measurement will be dependent upon equipment used; $\left(\mathrm{BV}_{\mathrm{DSS}}\right) \pm 1 \%$. 


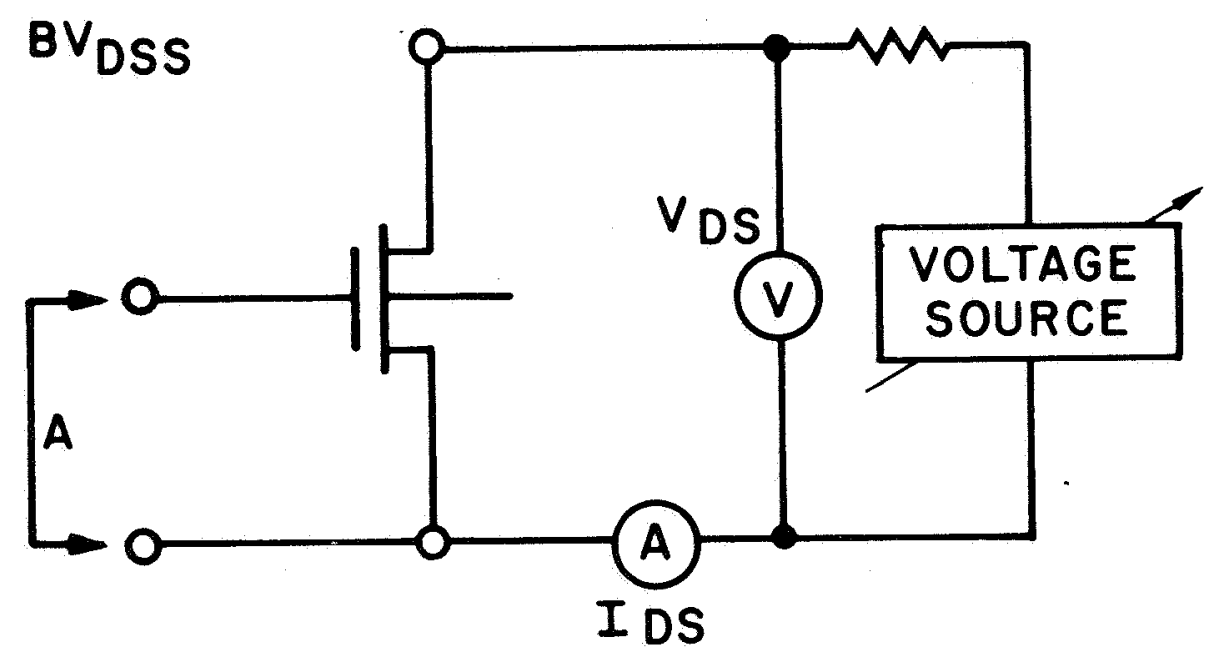

$A=$ SOURCE TO GATE SHORT CIRCUIT

Figure 1. BV DSS Measurement Setup 
Method 157

Method 157

Breakdown Voltage, Source to Gate (BV $\left.{ }_{G S S}\right)$

1. Purpose The purpose of this test is to determine if the breakdown voltage of the device under the specified conditions is greater than the specified minimum limit.

2. Test Circuit

See Figure 1.

3. Procedure

The resistor $R_{1}$ is a current-limiting resistor and should be of sufficiently high resistance to avoid excessive current flowing through the device and current meter. The voltage shall be gradually increased, with the specified condition ( $A, B, C$ or $D$ ) applied, from zero until either the minimum limit for BVSGX or the specified test current is reached. The device is acceptable if the minimum limit for $\mathrm{BV}_{\mathrm{SGX}}$ is reached before the test current reaches the specified value. If the specified test current is reached first, the device is rejected.

\section{Summary}

The following details shall be specified in the individual specification:

(a) Test current (See 3.)

(b) Bias condition:

A - Drain to Gate - Reverse bias. (Specify bias voltage).

B - Drain to Gate - Resistance return. (Specify resistance of $\mathrm{R}_{2}$ ). 


$$
\begin{aligned}
& \text { C - Drain to Gate - Short circuit. } \\
& \text { D - Drain to Gate - Open circuit. }
\end{aligned}
$$

\section{0 Accuracy}

The accuracy of measurement will be dependent upon equipment used; $B V_{G S S} \pm 1 \%$ with optimum equipment. 


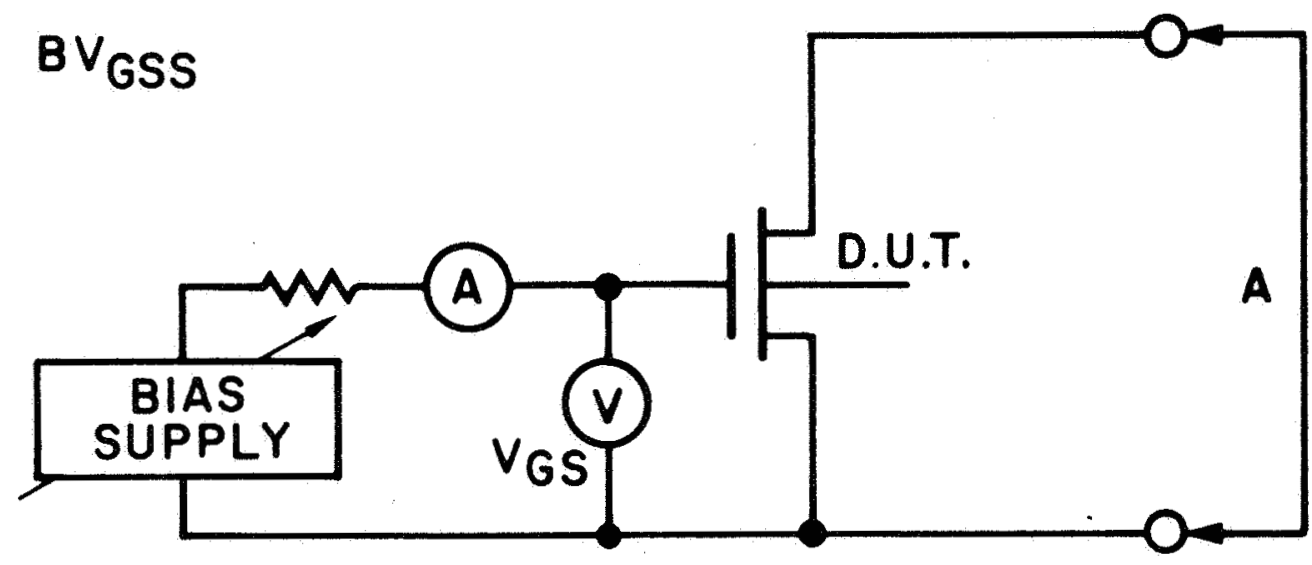

A-SHORT CIRCUIT SOURCE TO DRAIN

Figure 1. BV GSS Measurement Setup 


\section{Method 158 \\ Drain to Source Cutoff Current ( ${ }_{\text {DSS }}$ )}

1. Purpose The purpose of this test is to measure the cutoff current of the device under the specified conditions.

2. Test Circuit

See Figure 1.

3. Procedure

The specified voltage shall be applied between the drain and source with the specified test condition (condition A, B, C, or D) applied to the base. The measurement of current shall be made at the specified ambient or case temperature.

4. Summary

The following details shall be specified in the individual specification:

(a) Test voltage (see 3)

(b) Test temperature if other than $25^{\circ} \pm 3^{\circ} \mathrm{C}$ and whether case or ambient (see 3)

(c) Bias condition:
A - Source to Gate - Reverse bias (specify bias voltage).
$B$ - Source to Gate - Resistance return (specify resistance value of $R_{2}$ ).
C - Source to Gate - Short circuit.
D - Source to Gate - Open circuit. 
Method 158

\section{0 Accuracy}

The accuracy of measurement will be dependent upon equipment used; $I_{D S S} \pm 2 \%$ with optimum equipment. 


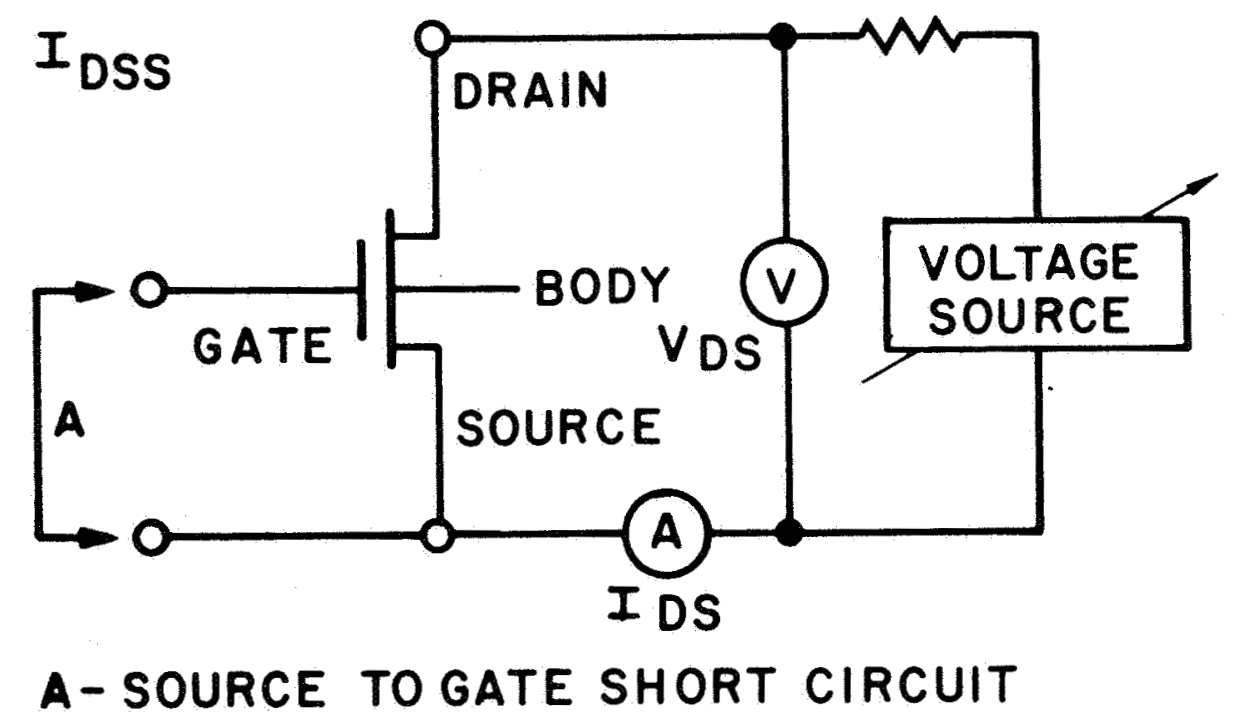

Figure 1. I $I_{\text {DSS }}$ Measurement Setup 
Method 159

Source to Gate Cutoff Current ( ${ }_{\text {GSS }}$ )

1. Purpose The purpose of this test is to measure the cutoff current of the device under the specified conditions.

2. Test Circuit

See Figure 1 .

3. Procedure

The specified direct current voltages shall be applied between the emitter and the base with the specified bias condition (condition A, B, C, or D) applied to the collector. The measurement of current shall be made at the specified ambient or case temperature.

4. Summary

The following details shall be specified in the individual specification:

4. 1 Test voltage (See 3.).

4. 2 Test temperature if other than $25^{\circ} \pm 3^{\circ} \mathrm{C}$ and whether case or ambient (See 3.).

4. 3 Bias condition (condition A, B, C, or D) :
A - Drain to Gate - Reverse bias (specify bias voltage).
$B$ - Drain to Gate - Resistance return (specify resistance of $\mathrm{R}_{2}$ ).
C - Drain to Gate - Short circuit.
D - Drain to Gate - Open circuit.

\section{0 Accuracy}

The accuracy of measurement will be dependent upon equipment used; $I_{G S S} \pm 3 \%$ with optimum equipment. 


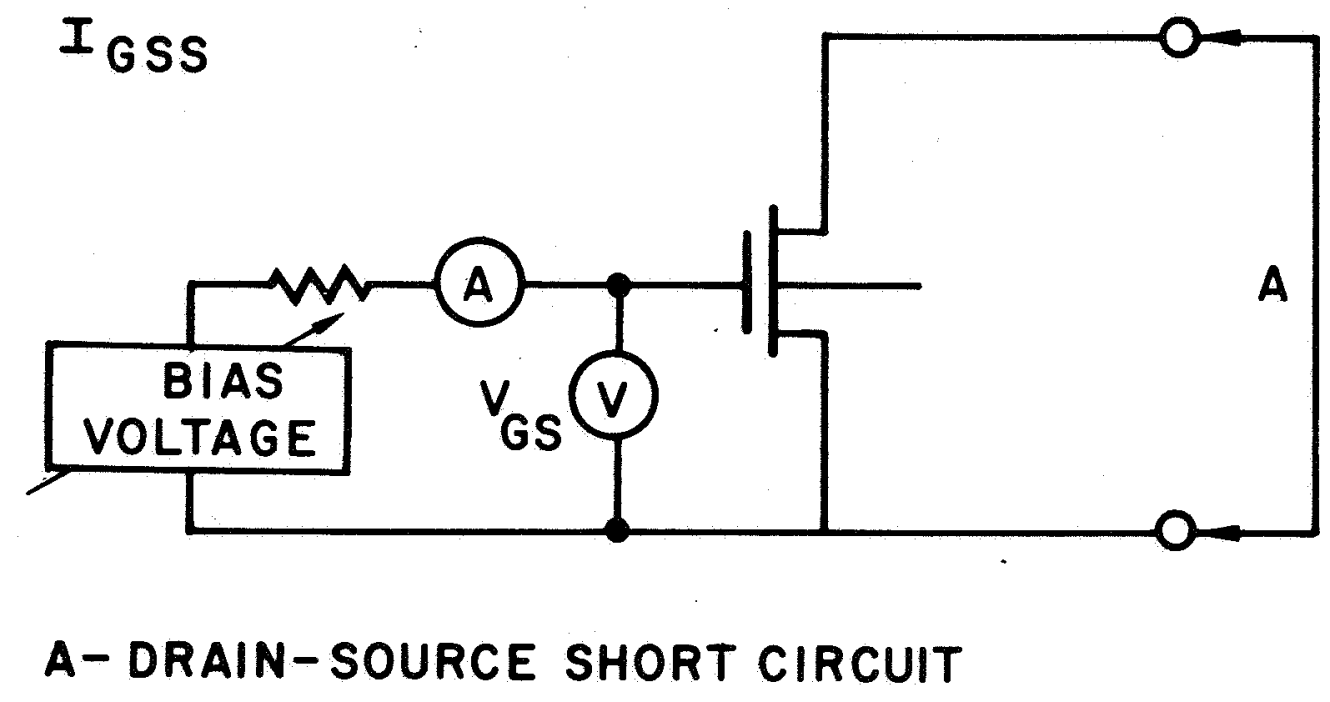

Figure 1. I $I_{\text {GSS }}$ Measurement Setup 
Method 160

Gate to Source Threshold Voltage ( $\left.\mathrm{V}_{\mathrm{GST}}\right)$

1. Purpose This method is designed to measure the voltage that will turn device on under specified conditions.

2. Test Circuit

See Figure 1.

3. Procedure

The gate voltage is increased until the specified drain current is obtained. The voltage read from source to gate at specified conditions. This will be the desired voltage.

\section{Summary}

The following details shall be specified in order to perform test.

(a) Test voltages or currents

(b) Parameters to be measured

\section{0 Accuracy}

The accuracy of measurement will be dependent upon equipment used; $\mathrm{V}_{\mathrm{GST}} \pm 1 \%$ with optimum equipment. 


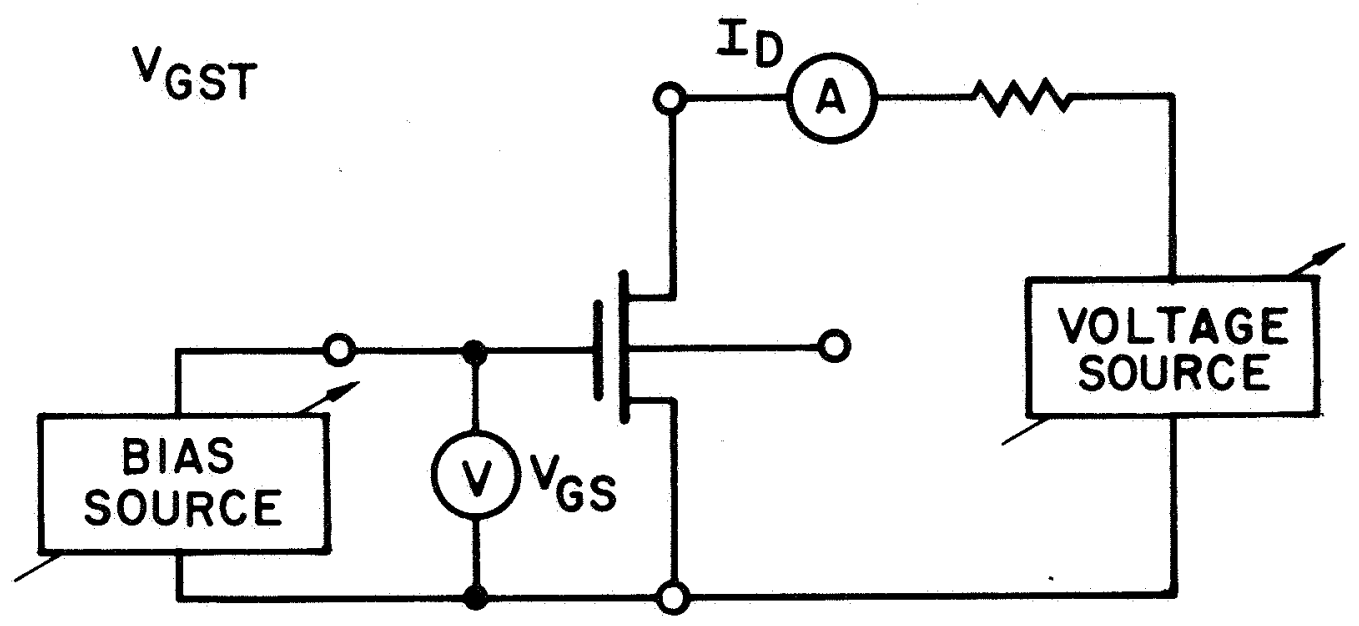

Figure 1. V GST Measurement Setup 
Method 161

Gate Leakage Resistance $\left(\mathrm{R}_{\mathrm{GS}}\right)$

1. Purpose The purpose of this method is to measure the input resistance of the device under the specified conditions.

2. Test Circuit.

See Figure 1.

3. Procedure

The resistor $R$ shall be made large. The ammeter shall be capable of reading currents to very low limits. The specified voltage shall be applied from source to gate. The leakage current will be read on ammeter therefore:

$$
\mathrm{R}_{\mathrm{GS}}=\frac{\mathrm{v}_{\mathrm{GS}}}{\mathrm{I}_{\mathrm{GS}}}
$$

\section{Summary}

The following details shall be specified.
(a) Test voltage.
(b) Test temperature.
(c) Specify ' $R$ ' 


\section{0 Accuracy}

The accuracy of measurement will be dependent upon equipment used; $\mathrm{R}_{\mathrm{GS}} \pm 1 \%$ with optimum equipment. 


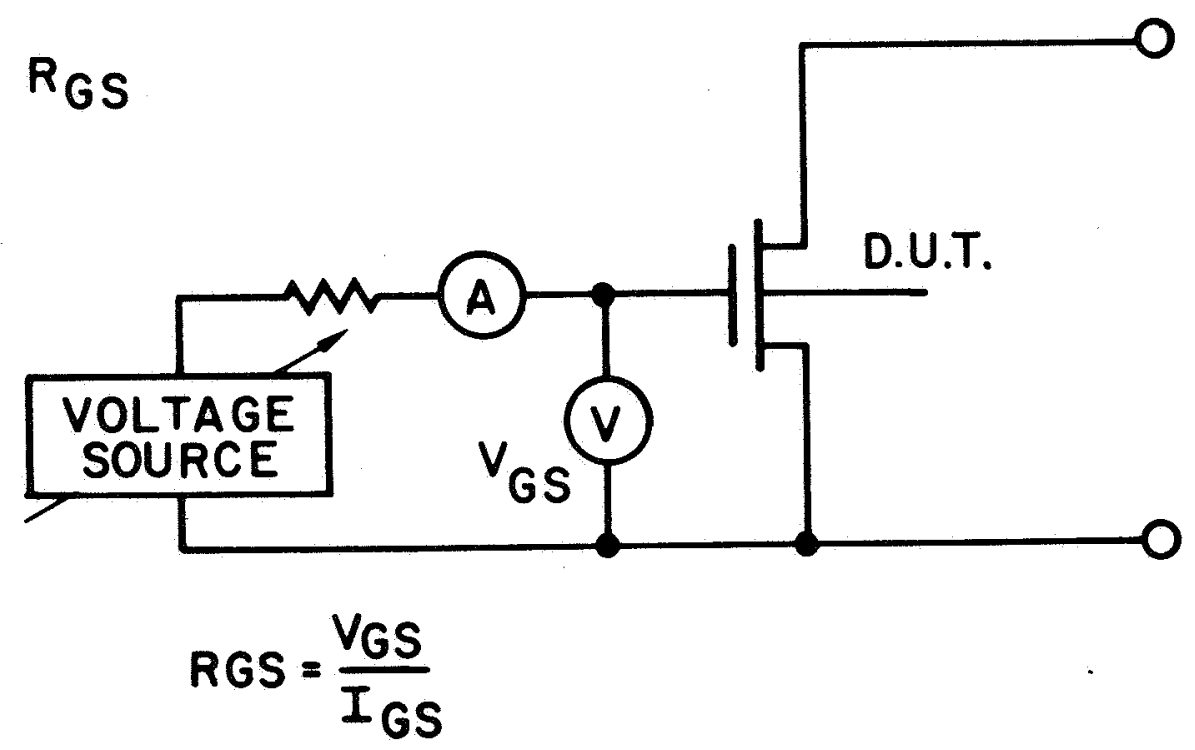

Figure 1. $\mathrm{R}_{\mathrm{GS}}$ Measurement Setup 
1. Purpose

The purpose of this method is to measure the dynamic resistance from drain to source at specified conditions.

\section{Test Circuit}

See Figure 1.

3. Procedure

The specified D. C. drain to source voltage shall be applied. D. C. gate to source voltage should be applied according to specification. With specified D. C. biases and voltages, an A. C. signal of a given voltage $r$ current is applied to source and drain. The measured A. C. voltage drop from drain to source, in conjunction with $\mathrm{A}$. C. drain to source current will determine $r d(o n)$

$$
R_{d(o n)}=\frac{V_{A . C .}}{I_{A . C .}}
$$

\section{Summary}

The following details shall be specified.
(a) D. C. voltage or current
(b) A. C. voltage or current
(c) Frequency of A. C. signal 


\section{0 Accuracy}

The accuracy of measurement will be dependent upon equipment used; $\mathrm{Rd}_{(\mathrm{on})} \pm 2 \%$ with optimum equipment. 


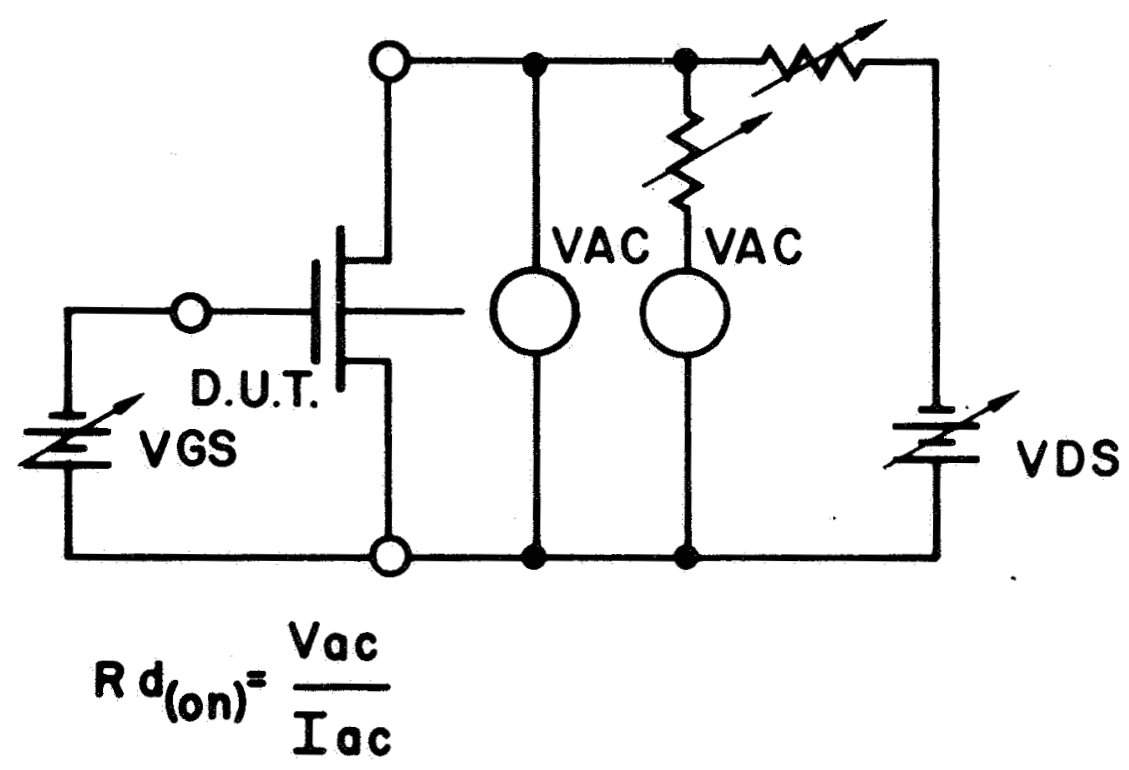

Figure 1. Rd(on) Measurement Setup 
Static Transconductance $\left(\mathrm{g}_{\mathrm{m}}\right)$

1. Purpose The purpose of this method is to measure the static transconductance of the device under specified conditions.

2. Test Circuit

See Figure 1.

3. Procedure

The resistor $R$, shall be made large. The resistor $R_{2}$ shall be chosen in combination with $V_{D D}$ so that the specified collector current is achieved at a value of $V_{D D}$ which is lower than $B V_{D S}$. The gate voltage shall be adjusted until $V_{D S}$ and $I_{D}$ achieve their specified values. The current $I_{D}$ or $I_{S}$ and the voltages $V_{G S}$, $\mathrm{V}_{\mathrm{GD}}$, or $\mathrm{V}_{\mathrm{SG}}$ shall then be measured. Using the values obtained through these measurements, the static transconductance shall be calculated as follows:
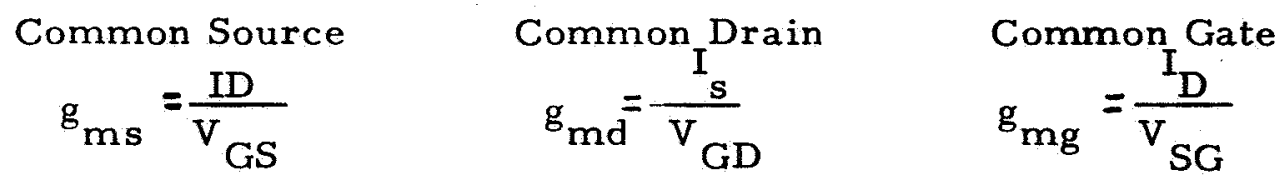

If high current values are to be used in the measurement, suitable pulse techniques may be used to provide pulses of short duty cycle to minimize the rise in junction temperature.

\section{Summary}

The following details shall be specified.

(a) Test voltage or current.

(b) Duty cycle and pulse width, if applicable. 
Method 163

\section{0 Accuracy}

The accuracy of measurement will be dependent upon equipment used; $\mathrm{g}_{\mathrm{m}} \pm 1 \%$ with optimum equipment. 
Method 163

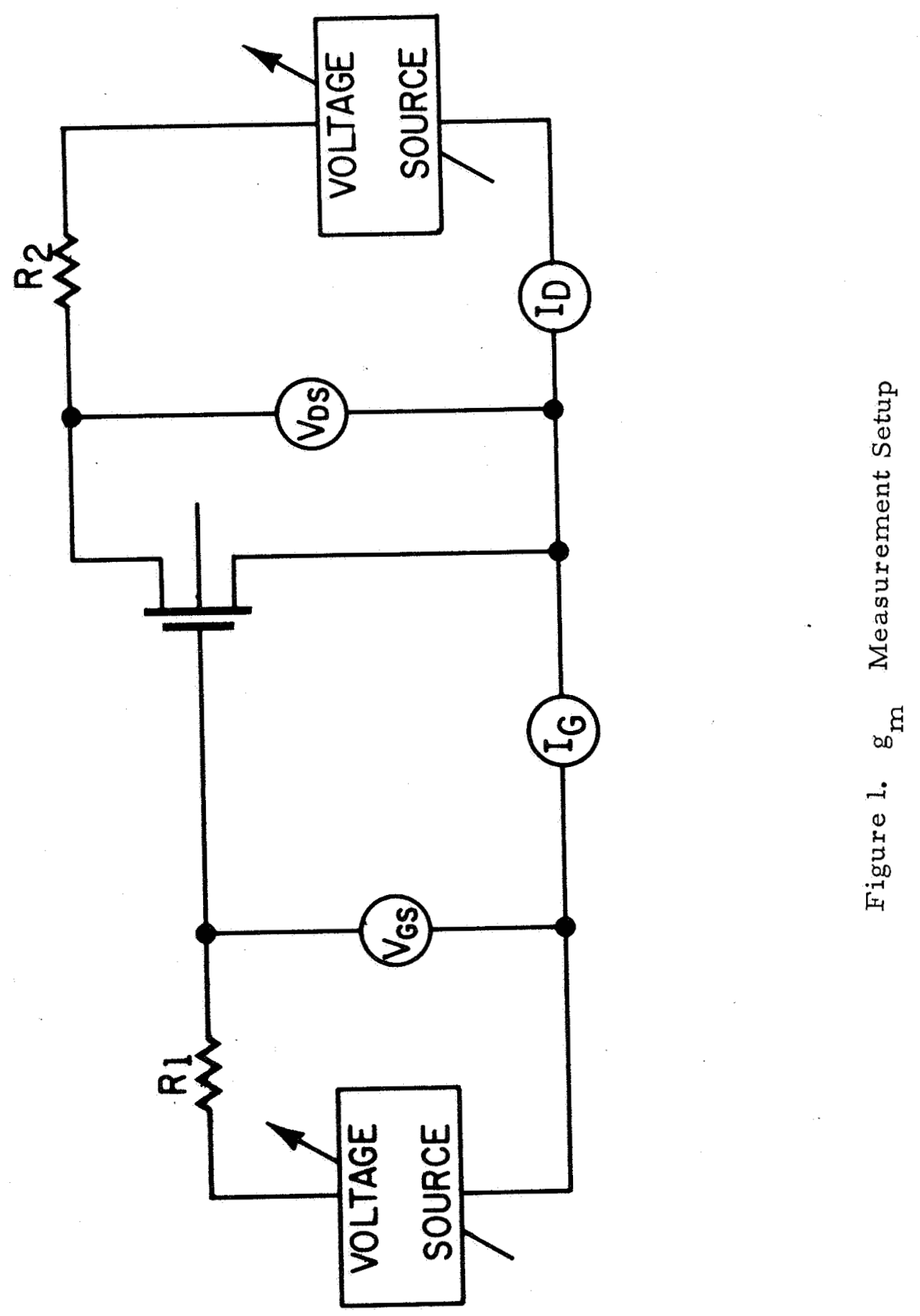


Direct Inter-terminal Capacitance $\left(C_{G S}, C_{G D}, C_{D S}\right)$

1. Purpose The purpose of this test is to measure the direct inter-terminal capacitance between specified terminals using specified electrical biases.

2. Test Circuit

A direct capacitance bridge or resonance Method may be used to determine the value of the direct inter-terminal capacitance. See Figures 1 through 3.

\section{Procedure}

The direct inter-terminal capacitance can be determined by using Method A or Method B.

3. 1 Method A The specified voltage shall be applied between specified terminals: an a.c. small signal shall be applied to the terminals and the direct inter-terminal capacitance shall be measured. The lead capacitance beyond $1 / 2$ inch from the body seat shall be effectively eliminated by suitable means such as test socket shilding. The abbreviations and symbols used are defined as follows:

$C_{D G}($ dir) ... Drain to Gate inter-terminal direct capacitance.

$\mathrm{C}_{\mathrm{SG}}(\mathrm{dir}) \ldots$ Drain to Gate inter-terminal direct capacitance.

$\mathrm{C}_{\mathrm{DS}}(\mathrm{dir})$... Drain to Gate inter-terminal direct capacitance.

3.2 Method B A suitable resonance method can be utilized to measure the following two-terminal capacitances: 
$C_{1}$ - Capacitance between drain terminal and ground, with gate and source terminals grounded.

$\mathrm{C}_{2}$ - Capacitance between the gate terminal and ground, with drain and source terminals grounded.

$\mathrm{C}_{3}$ - Capacitance between the drain and gate terminals strapped together and ground, with the source terminal grounded.

The direct inter-terminal capacitance can than be calculated from the following relationship:

$$
C_{D G}=\frac{C_{1}+C_{2}-C_{3}}{2}
$$

The direct inter-terminal capacitance for other configurations can be determined by suitable modifications of the above procedure. Such modifications shall be capable of demonstrating device conformance to the minimum requirements of the individual specification.

\section{Simmary}

The following details shall be specified on the individual specification:

(a) Terminal arrangement

(b) D. C. biasing conditions

(c) Test voltage or current.

(d) Measurement frequency.

\section{0 Accuracy}

The accuracy of measurement will be dependent upon equipment used; $C_{X X} \pm 1 \%$ with optimum equipment. 
Method 164

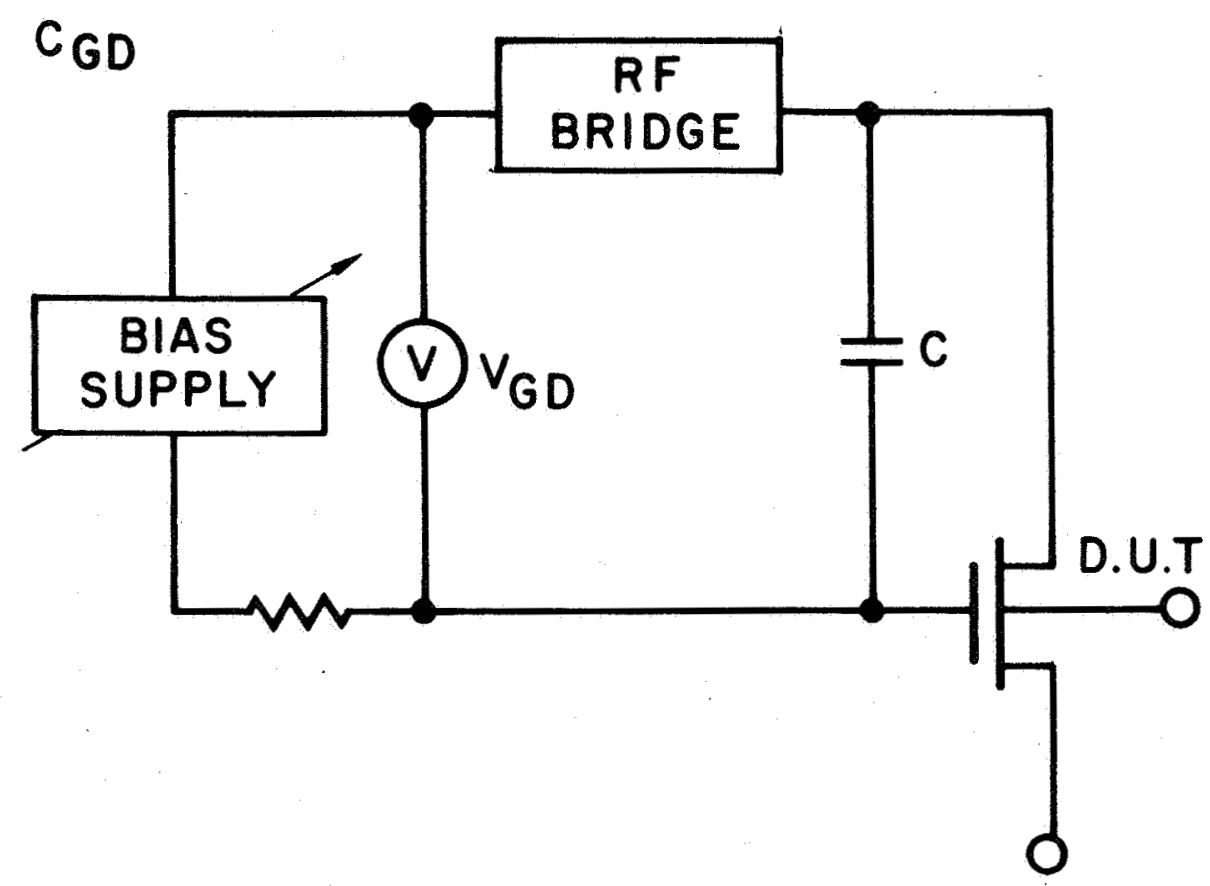

Figure 1. $\mathrm{C}_{\mathrm{GD}}$ Measurement Setup

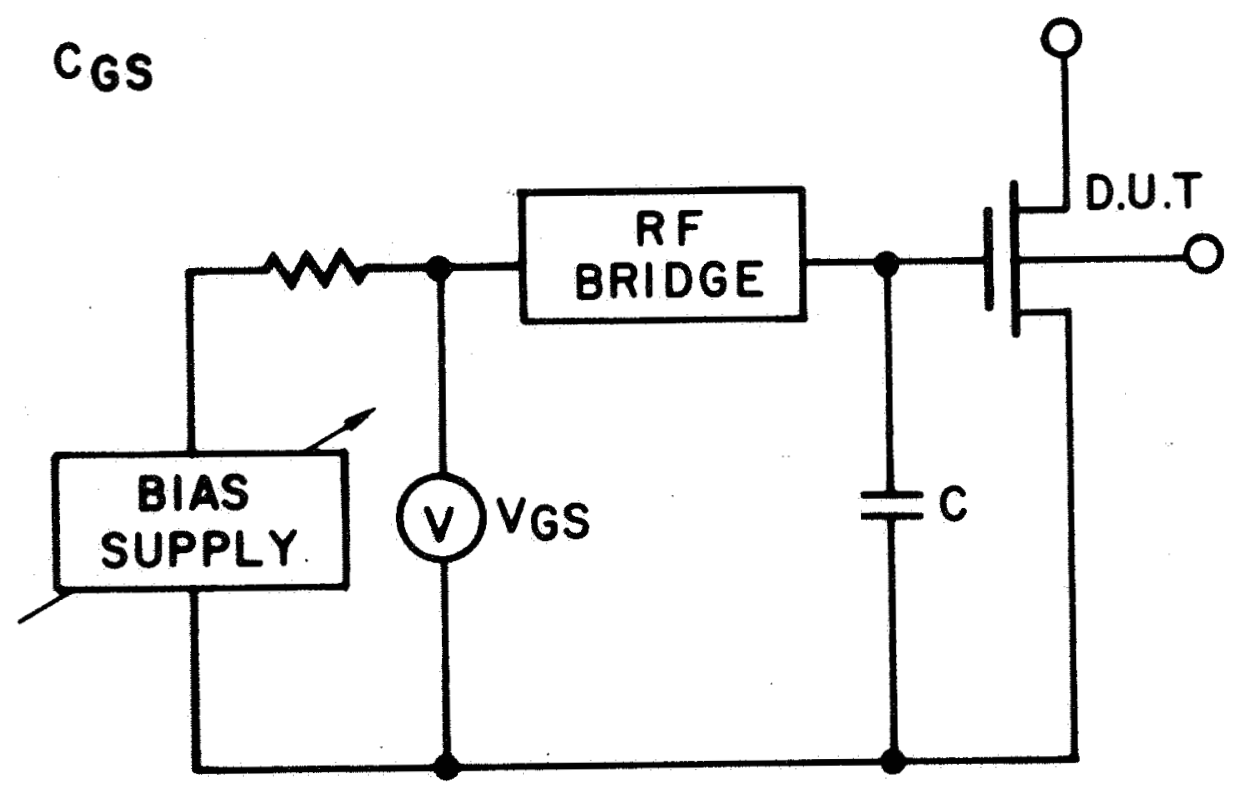

Figure 2. $C_{G S}$ Measurement Setup 
$C_{D S}$

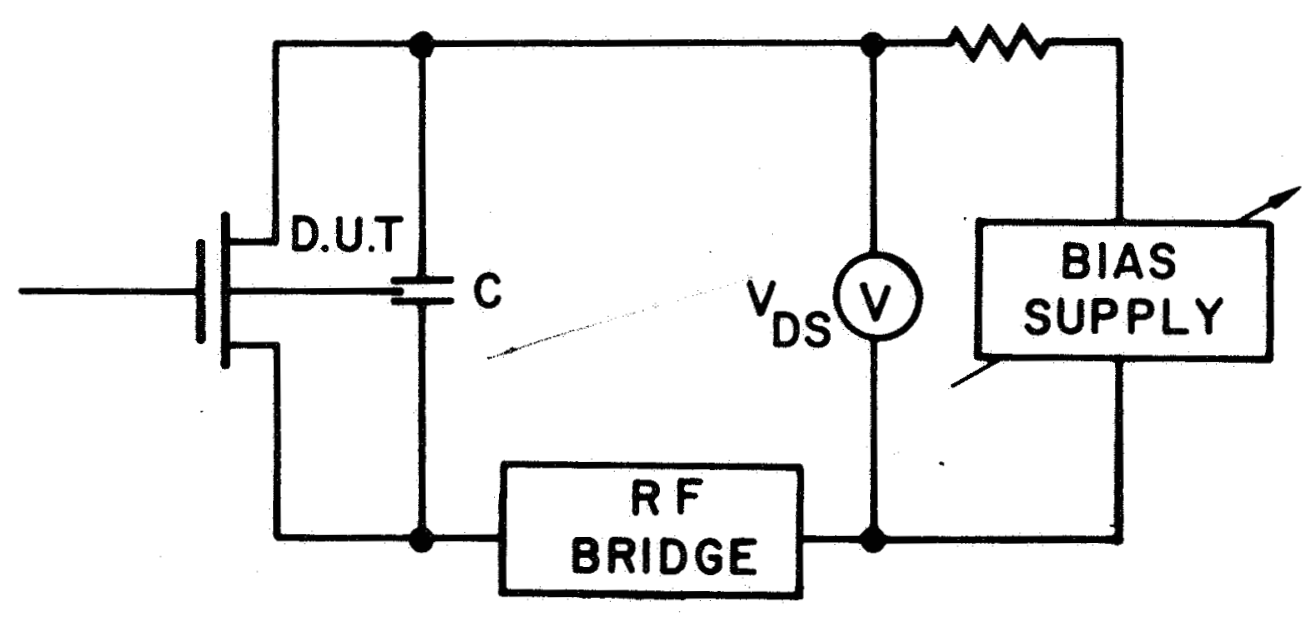

- Figure 3. $\mathrm{C}_{\mathrm{DS}}$ Measurement Setup 